$$
\begin{aligned}
& \text { GA-A21186-VO/ } 2 \\
& \text { UC-420 }
\end{aligned}
$$

\title{
DIII-D RESEARCH OPERATIONS
}

\author{
ANNUAL REPORT TO THE \\ U.S. DEPARTMENT OF ENERGY
}

OCTOBER 1, 1991 THROUGH SEPTEMBER 30, 1992

\author{
by \\ PROJECT STAFF \\ D. BAKER, Editor
}

Work prepared under

Department of Energy

Contract DE-AC03-89ER51114

GENERAL ATOMICS PROJECTS 3466/3467/3470/3473

DATE PUBLISHED: MAY 1993
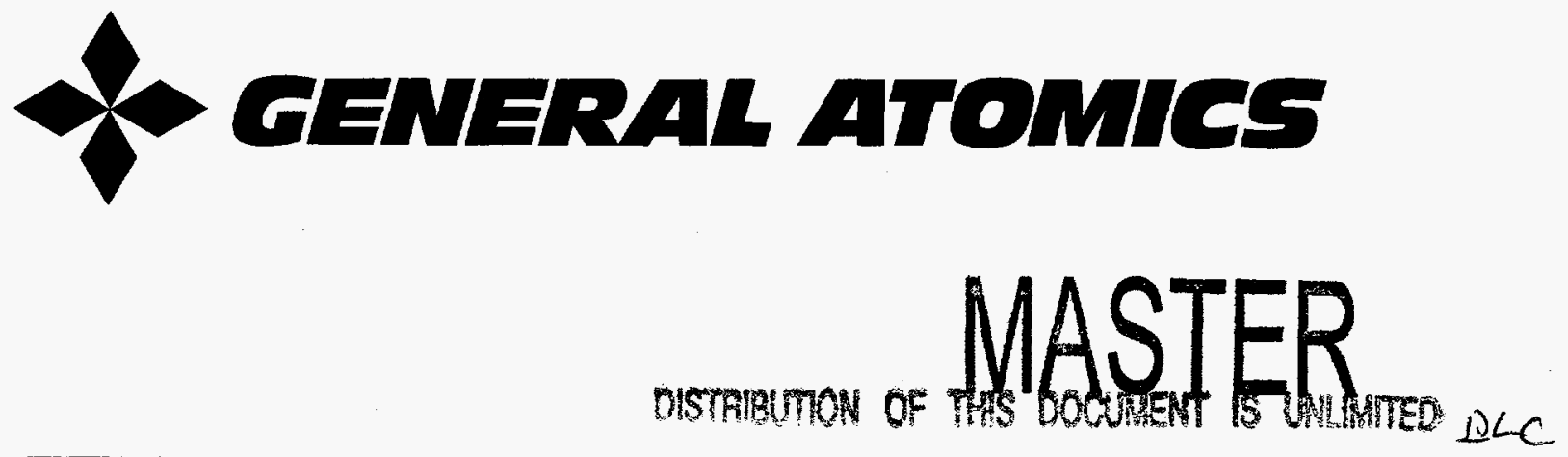


\section{DISCLAIMER}

This report was prepared as an account of work sponsored by an agency of the United States Government. Neither the United States Government nor any agency thereof, nor any of their employees, makes any warranty, express or implied, or assumes any legal liability or responsibility for the accuracy, completeness, or usefulness of any information, apparatus, product, or process disclosed, or represents that its use would not infringe privately owned rights. Reference herein to any specific commercial product, process, or service by trade name, trademark, manufacturer, or otherwise, does not necessarily constitute or imply its endorsement, recommendation, or favoring by the United States Government or any agency thereof. The views and opinions of authors expressed herein do not necessarily state or reflect those of the United States Government or any agency thereof.

This report has been reproduced directly from the best available copy.

Available to DOE and DOE contractors from the Office of Scientific and Technical Information

P.O. Box 62

Oak Ridge, TN 37831

Prices available from (615) 576-8401, FTS 626-8401.

Available to the public from the Netional Technical Information Service

U.S. Department of Commerce 5285 Port Royal Rd.

Springfield, VA 22161 


\section{TABLE OF CONTENTS}

1. DIII-D PROGRAM OVERVIEW …................................................................ $1-1$

1.1. Introduction ................................................................................ $1-1$

1.2. Highlights of the FY92 DIII-D Research Program ................................... 1-5

1.2.1. Divertor Radiation...................................................................... $1-5$

1.2.2. Improved Understanding of VH-Mode ........................................... $1-6$

1.2.3. Fast Wave Current Drive ................................................................ 1-6

1.2.4. Toroidal Alfvèn Eigenmodes .......................................................... $\quad 1-9$

2. DIVERTOR AND BOUNDARY RESEARCH PROGRAM..................................... 2-1

2.1. Overview .................................................................................. 2-1

2.2. Divertor Physics ................................................................................... 2-2

2.2.1. Divertor and SOL Scaling Studies ................................................ 2-2

2.2.2. Scrape-off Layer Measurements .................................................... 2 2-6

2.2.3. Radiative Divertor .................................................................... 2-7

2.2.4. Heat Flux Profile Structures and Toroidal Asymmetries ................ 2- 2-8

2.2.5. Lithium Beam Edge Diagnostic .................................................. 2-10

2.3. Modeling and Edge Database ................................................................. 2-10

2.3.1. SOL Modeling....................................................................... 2-10

2.3.2. Edge Database ............................................................................ $\quad 2-12$

2.4. Impurity Transport and Control ............................................................. 2-14

3. ADVANCED TOKAMAK STUDIES..........................................................

3.1. Overview ................................................................................... $3-1$

3.2. H-Mode Confinement ............................................................................. 3-2

3.3. Improved Confinement through Modification of the Current Profile .......... 3-8

3.4. VH-Mode ......................................................................................... 3-11

3.5. Noninductive Current Drive ................................................................... 3-21

3.5.1. Electron Cyclotron Heating for Fast Wave Current Drive

3.5.2. Fast Wave Heating and Current Drive ........................................... 3-21

3.5.3. Fast Wave Current Drive ............................................................... 3-22

4. TOKAMAK PHYSICS..............................................................................

4.1. Overview .......................................................................................... 4-1

4.2. Toroidal Alfvèn Eigenmodes and Other Global Alfvèn Modes ....................... 4 4-1

4.3. Magnetic Braking for Study of the Sole of Rotation on

Turbulence and Confinement. 
4.4. Dimensionless Variable Scaling Experiments

4.5. Confinement Scaling with Electron Cyclotron Heating ................................ 4-17

4.6. Theory of Plasma Particle and Heat Pinches ................................................. 4-18

4.7. Experimental Studies of the Heat Pinch ...................................................... 4-21

4.7.1. Introduction ............................................................................ 4-21

4.7.2. Off-Axis ECH Experiments .......................................................... 4-21

4.7.3. Off-Axis NBI Heating Experiments................................................ 4-23

4.8. Fast Wave Electron Heating Without the Faraday Shield............................. 4-27

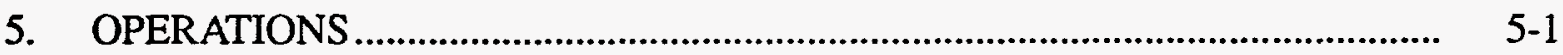

5.1. Tokamak Operations ........................................................................ $5-1$

5.1.1. Operations Summary ....................................................................... 5-1

5.1.2. In-Vessel Work .............................................................................. $5-1$

5.1.3. Vessel Conditioning......................................................................... $5-5$

5.2. Neutral Beam Operations............................................................................. 5-6

5.2.1. Operations Summary ....................................................................... 5-6

5.2.2. System Improvement and Maintenance ........................................... 5- 5

5.2.3. System Availability ........................................................................... $5-8$

5.3. Electron Cyclotron and Ion Cyclotron Heating Operations.......................... 5-10

5.3.1. Electron Cyclotron Heating Operations .......................................... 5-10

5.3.2. Fast Wave Heating and Current Drive Operations............................ 5-10

5.4. Disruption Studies and Plasma Control ........................................................ 5-11

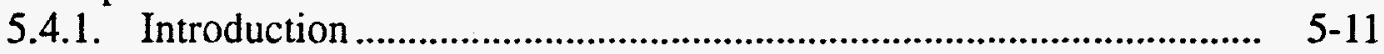

5.4.2. Plasma Control ...................................................................... $5-16$

5.5. Diagnostics.................................................................................... 5-20

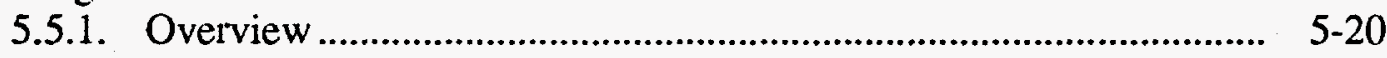

5.5.2. New or Upgraded Diagnostics ........................................................ 5-20

5.5.3. Diagnostics Under Development ...................................................... 5-27

5.6. Reliability and Availability ................................................................ 5-28

5.6.1. Integrated Preventive Maintenance Program ................................. 5-28

5.6.2. Significant Event Review ........................................................... 5-29

5.7. Radiation Management .................................................................... $5-30$

5.8. Electrical Engineering......................................................................... $5-32$

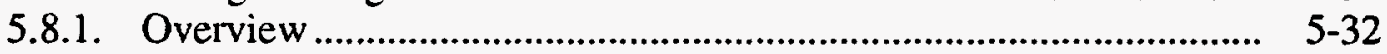

5.8.2. Operation Support ...................................................................... $5-32$

5.8.3. Coil Power Systems .................................................................... $5-33$

5.8.4. High Voltage Systems ............................................................... $5-33$

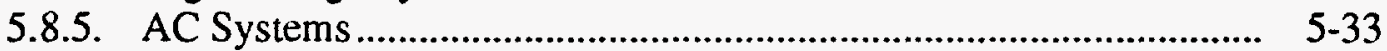

5.8.6. Instrumentation and Control Systems.............................................. 5-34

5.8.7. Preventive Maintenance ............................................................ 5-34

5.9. Mechanical Engineering............................................................... 5-34

5.9.1. Tokamak Systems .................................................................. $5-34$

5.9.2. Fluid Systems ............................................................................... $5-38$

5.10. FY92 Computer Data Systems ............................................................ $\quad 5-40$

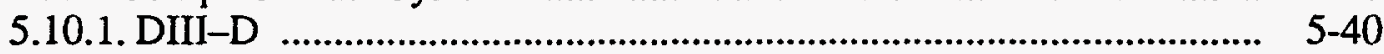

5.10.2. Computer Hardware Upgrades .......................................................... 5-40

5.10.3. Software Improvements....................................................................... 5-41 
5.10.4. Remote Access for Off-Site Collaboration........................................ 5-42

5.10.5. Computer Replacement Plan ...................................................... $5-42$

5.10.6. Preventive Maintenance Tracking Program Improved ...................... 5-43

5.10.7. New CAD System Betler Supports DIII-D .................................... 5-44

5.10.8. Plan for Computer Connection to Russia ....................................... 5-44

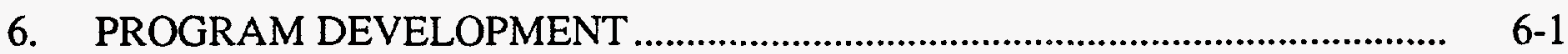

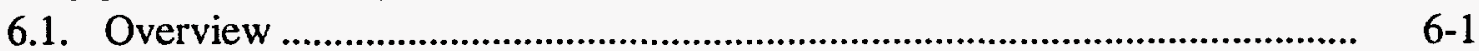

6.2. Advanced Divertor Program.................................................................. 6-2

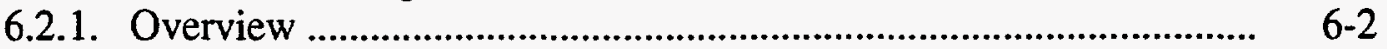

6.2.2. Thermal Analysis of the Divertor Cryopump..................................... $6-4$

6.3. $110 \mathrm{GHz}$ ECH System ............................................................................... 6-6

7. SUPPORT SERVICES …..................................................................................

7.1. Quality Assurance ............................................................................

7.1.1. Design Support ........................................................................ 7-1

7.1.2. Inspection Support .......................................................................... 7-1

7.1.3. As-Built Measurement Support ...................................................... 7-2

7.1.4. Optical Tooling/Layout/Alignment Support.................................... $\quad 7-3$

7.1.5. QA System Improvements............................................................. 7-3

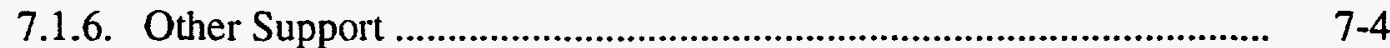

7.2. Planning and Control ..............................................................................

7.3. Environment Safety and Health..................................................................

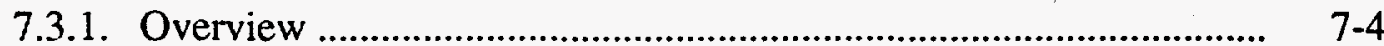

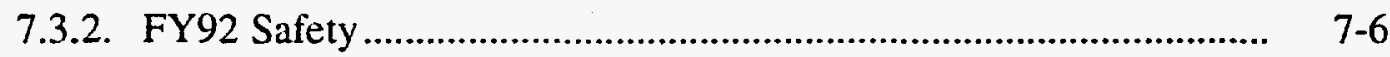

7.3.3. Radiation Safety ........................................................................ 7-10

7.3.4. FY92 Radiation Safety .............................................................. 7-11

7.4. Visitor and Public Information Program ...................................................... 7-14

8. CONTRIBUTION TO ITER PHYSICS R\&D ...................................................

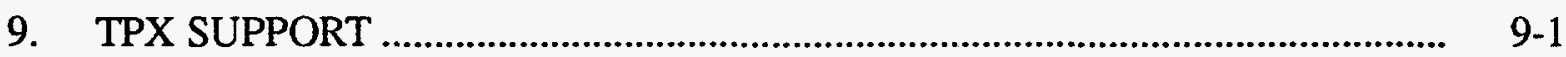

9.1. Programmatic Activities .......................................................................... 9-1

9.2. Physics and Machine Operations........................................................... 9-1

9.3. Divertor Studies...................................................................................... $9-2$

9.4. Component Engineering ................................................................................ 9-3

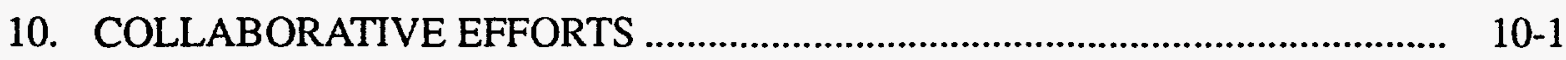

10.1. DIII-D Collaboration Programs Overview ................................................ 10-1

10.1.1. Japan Atomic Energy Research Institute ........................................... 10-1

10.1.2. National Laboratories .................................................................. $10-2$

10.1.3. University Programs ................................................................ $10-9$

10.2. International Cooperation ....................................................................... 10-10

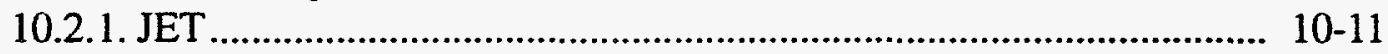


10.2.2. ASDEX-U

10.2.3. Tore Supra.

\section{LIST OF FIGURES}

1.1-1. Cross section of DIII-D with flux surfaces of a double-null divertor discharge superimposed

1.2-1. Peaked heat flux is fairly independent of both $D_{2}$ injection rate and injection location, when plotted versus neutral pressure in the midplane and divertor

1.2-2. Variation of energy confinement time enhancement factor with average triangularity of the separatrix flux surface for single- and double-null discharges.

1.2-3. Current drive efficiency $\gamma$ for the same discharges as in Fig. 3.5-3 as a function of central electron temperature

1.2-4. TAE frequency dependence on $\mathrm{v}_{\mathrm{A}}$, observed and calculated frequencies at increasing toroidal magnetic field.

2.2-1. Scaling of the peak heat flux to the divertor for ELMing H-mode plasmas ...

2.2-2. Electron temperature and electron density of the outer strike point versus input power.

2.2-3. Density profiles in the SOL for $\mathrm{L}-, \mathrm{H}-$, and $\mathrm{VH}-$ modes, measured by the SNLA/UCLA probe

2.2-4. Particle transport in the SOL as measured by the SNLAVUCLA probe.

2.2-5. Peak heat flux to the divertor is reduced as the midplane pressure increases..

2.2-6. Evolution of the DIII-D divertor target plate heat flux profile during a double-null VH-mode.

2.3-1. Radial profile for (a) electron density, and (b) electron and ion temperature 
2.3-2. Scaling relationships are extracted from the boundary database BNDY

2.4-1. Parameters during a shot which attains VH-mode; interferometric measured $n_{e}, D_{\alpha}$, spectroscopic signals, $\tau_{E}$, and the injected and radiated power

2.4-2. Contour plot of scrape-off $T_{e}$ and $n_{e}$ as a function of effective radial localities $r / a=\rho$ and time, along with the results of the $2 D$ penetration model for carbon, oxygen and nickel, showing the fraction of launched neutral $\mathrm{C}, \mathrm{O}, \mathrm{Ni}$ particles that make it past the separatrix.

2.4-3. The energy confinement time, normalized to JET/DIII-D scaling versus input power

2.4-4. Energy confinement increases with lower particle fueling influx

3.2-1. Plots of various plasma profiles in DIII-D near the plasma edge at times relative to the $\mathrm{L}-\mathrm{to}-\mathrm{H}$ transition.

3.2-2. Time history across the L-to-H transition in DIII-D of the divertor $\mathrm{D}_{\alpha}$ signal; edge poloidal rotation, toroidal rotation and inferred $\mathrm{E}_{\mathrm{r}}$ from charge exchange spectroscopy; and reflectometer power.

3.2-3. Time history across the L-to-H transition in DIII-D of edge ion temperature gradient, C VII density gradient, C VII pressure gradient and most negative edge $E_{r}$

3.3-1. For a discharge with a current ramp, (a) the single fluid diffusivity and (b) the flux surface averaged poloidal field versus time at $\rho=0.5$ and $\rho=0.8$

3.3-2. Radial electric field versus normalized radius for an elongation ramp, $\mathrm{H}$-mode discharge at different times during the current profile relaxation phase

3.4-1. Variation of energy confinement time enhancement factor with average triangularity of the separatrix flux surface for single- and double-null discharges.

3.4-2. Change in the radial electric field, $E_{1}, E \times B$ velocity shear, $V^{\prime} E \times B$, and effective thermal diffusivity, $\chi_{\text {eff }}$ during a $\mathrm{VH}-$ mode discharge.

3.4-3. Energy confinement time enhancement factor, $\tau_{E} / \tau_{J E T} / D I I I-D$ and quantities corresponding to the three models for confinement enhancement improvement considered

3.4-4. An increase in the shear in the toroidal rotation speed occurs near the time of a rapid jump increase in the energy confinement enhancement factor

3.4-5. Events associated with the termination of ELM-free VH-mode 3-19

3.5-1. (a) Behavior of an ECH-preheated discharge with co-FWCD 
3.5-2. Fraction of the total plasma current which is noninductively-driven as a function of the co-FWCD power level divided by lineaveraged density.

3.5-3. Current drive efficiency $\gamma$ for the same discharges as in Fig. 3.5-2 as a function of central electron temperature

4.2-1. TAE frequency dependence on $v_{A}$, observed and calculated frequencies at increasing toroidal magnetic field

4.2-2. Computed Alfvèn spectrum for discharge 71524 at $1875 \mathrm{msec}$

4.2-3. Computed growth rate for discharge 71524 at $1875 \mathrm{msec}$ as a function of toroidal mode number $\mathrm{n}$.

4.2-4. Power spectrum for discharge 75346 showing TAE and EAE spectra

4.2-5. Mode displacement plots for (a) TAE mode with $\omega^{2}=0.081$ and

(b) BAE mode with $\omega^{2}=0.072$

4.2-6. Observed frequency dependence with increasing $\beta$ for discharge 71524

4.2-7. Scaling of low frequency mode frequencies with Alfvèn speed $v_{A}$ showing computed BAE frequency for discharge 71524 at $2150 \mathrm{msec}$

4.2-8. Energy deposited in foil bolometers mounted on the vessel wall as a function of the position of an obstacle during TAE activity

4.4-1. Ratio of $1.99 \mathrm{~T}$ and $1.07 \mathrm{~T}$ one-fluid heat diffusivity versus normalized radius

4.5-1. Energy confinement time for fundamental and second harmonic ECH, corrected for the "conventional" $\mathrm{I} / \mathrm{P} 1 / 2$ scaling

4.7-1. Electron and ion temperature profiles during off-axis $\mathrm{ECH}$

4.7-2. Electron and ion thermal heat flux during off-axis ECH

4.7-3. Ion and electron thermal heat diffusivities for the case of off-axis NBI heating

4.7-4. Rapid time evolution of the electron temperature during off-axis NBI heating

4.8-1. Comparison of unshielded fast wave heating and $\mathrm{ECH}_{\text {for }} \mathrm{B}_{\mathrm{T}}=2 \mathrm{~T}$

5.1-1. DIII-D FY92 weekly operations schedule.

5.1-2. DIII-D machine availability

5.2-1. Neutral beam availability by month

5.2-2. Causes of downtime by category

5.4-1. A wide range of discharge shapes has been produced in DIII-D using the digital plasma control system 
5.4-2. Controlling plasma stored energy with the advanced control system

5.5-1. (a) The fan of view chords in the poloidal plane spans the interaction region with the vessel floor of the single-null divertor configuration.

6.1-1. DIII-D facility capabilities and upgrades

6.1-2. The DIII-D long range plan is a coherent integrated program

6.3-1. Initial $110 \mathrm{GHz}$ heating on DIII-D

10.1-1. Helium density profile as measured by CER just before and during a giant ELM

10.2-1. Field line puncture plot showing the poloidal structure of the ergodic boundary layer in Tore Supra

10.2-2. Left half - poloidal modulations in the background plasma parameters of Tore Supra due to the islands shown in Fig. 10.2-1 $10-14$

\section{LIST OF TABLES}

1.1-1 DIII-D Capabilities

1.1-2 DIII-D Achieved Parameters .

1.1-3 Collaborators Participating at DIII-D..

5.1-1 Major Vent Tasks.

5.5-1 Plasma Diagnostics

6.2-1 Cryopump Fatigue Life and Buckling Loads for 3 MA "Slow" Disruptions...

8-1 Planned DIII-D Contributions to ITER Long-Term Physics R\&D

8-2 Synopsis of DIII-D Contributions to ITER Long-Term Physics R\&D Needs. 
SECTION 1

\section{DIII-D PROGRAM OVERVIEW}




\section{DIII-D PROGRAM OVERVIEW}

\subsection{INTRODUCTION}

The DIII-D tokamak research program is carried out by. General Atomics (GA) for the U.S. Department of Energy (DOE). The DIII-D is the most flexible tokamak in the world. The primary goal of the DIII-D tokamak research program is to provide data needed by International Thermonuclear Experimental Reactor (ITER) and to develop a conceptual physics blueprint for a commercially attractive electrical demonstration plant (DEMO) that would open a path to fusion power commercialization. Specific DIII-D objectives include the steady-state sustainment of plasma current as well as demonstrating techniques for microwave heating, divertor heat removal, fuel exhaust and tokamak plasma control. The DIII-D program is addressing these objectives in an integrated fashion with high beta and with good confinement. The DIII-D long-range plan is organized into two major thrusts; the development of an advanced divertor and the development of advanced tokamak concepts. These two thrusts have a common goal: an improved DEMO reactor with lower cost and smaller size than the present DEMO which can be extrapolated from the conventional ITER operational scenario. In order to prepare for the long-range program, in FY92 the DIII-D research program concentrated on three major areas: Divertor and Boundary Physics, Advanced Tokamak Studies, and Tokamak Physics.

The major goals of the Divertor and Boundary Physics studies are the control of impurities, efficient heat removal and understanding the strong role that the edge plasma plays in the global energy confinement of the plasma. The advanced tokamak studies initiated the investigation into new techniques for improving energy confinement, controlling particle fueling and increasing plasma beta. The major goal of the Tokamak Physics Studies is the understanding of energy and particle transport in a reactor relevant plasma.

A cross sectional view of DIII-D (Fig. 1.1-1) shows a diverted configuration in which magnetic equilibrium forms a so-called double-null geometry. Several of the major machine 
Fig. 1.1-1. Cross section of DIII-D with flux surfaces of a double-null divertor discharge superimposed.

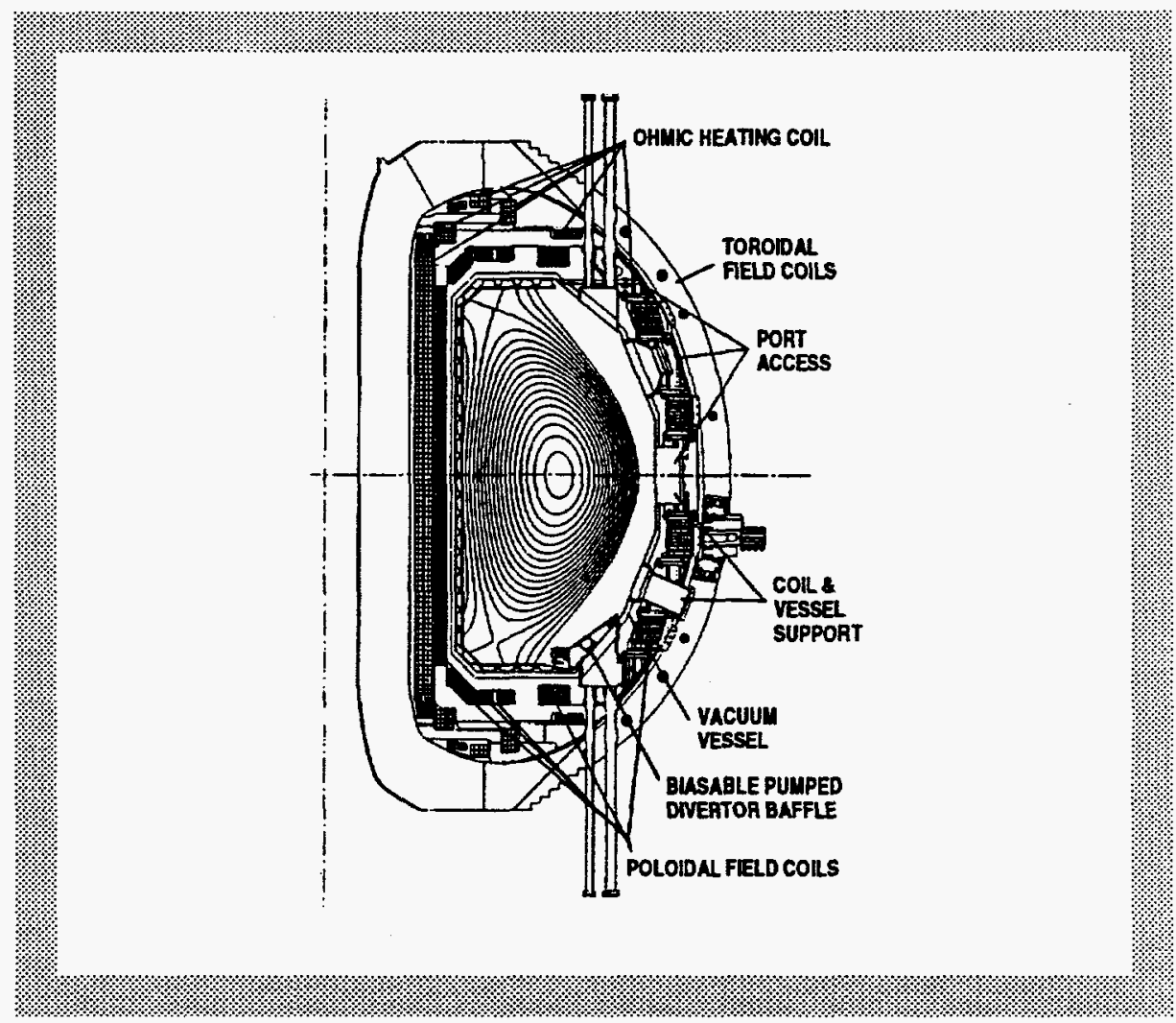

components are also called out in this figure. This equilibrium configuration is only one of many available in DIII-D. Because of this flexibility in shaping and positioning of the magnetic equilibrium, a broad range of plasma parameters are available for experimentation.

The DIII-D tokamak characteristics, maximum plasma parameters and experimental results are summarized in Tables 1.1-1 and 1.1-2. The experimental results, which were obtained from DIII-D, formed the basis for 11 substantial and significant papers presented at the International Atomic Energy Agency (IAEA) meeting at the end of the fiscal year.

To carry out the DII-D program, GA provides a combination of extensive institutional experience in the field, a skilled and experienced staff of international repute, and a uniquely flexible facility - the DIII-D tokamak. GA has had an active fusion research program for more than three decades. It is the only industrial participant in the U.S. program with a major, integrated effort in all aspects of plasma physics and fusion research, from basic plasma theory, magnetic fusion device design, engineering, construction, and operation, to fusion reactor technology. 
GA-A21186; FY92 DIII-D Research Operations Annual Report

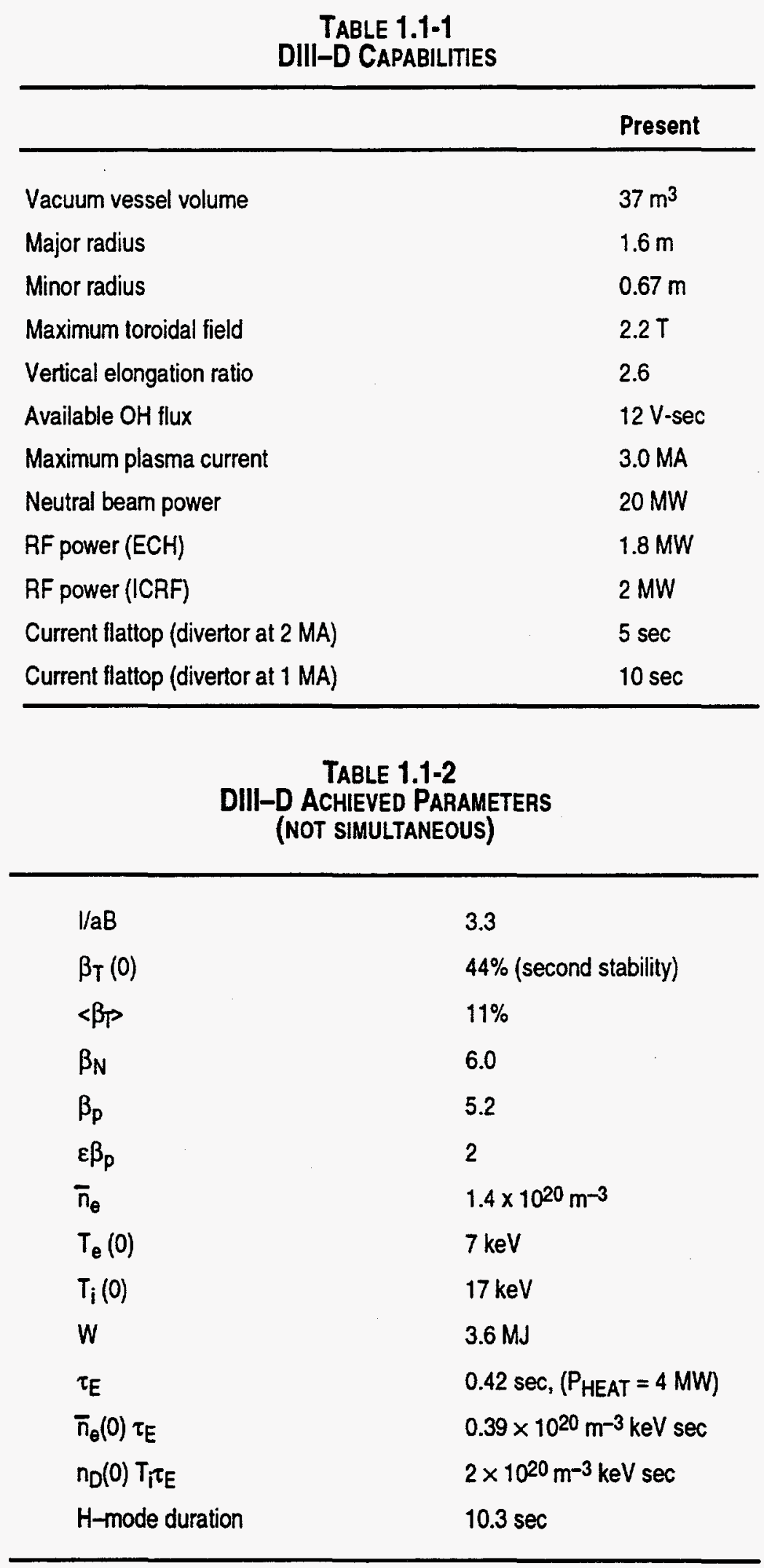


In addition, collaborations with other U.S. and international fusion programs are an essential feature of the DIII-D program. These collaborations assist and bring expertise to DIII-D. Principal among these efforts are the ongoing cooperative efforts with the Japan Atomic Energy Research Institute (JAERI), the Lawrence Livermore National Laboratory (LLNL), Oak Ridge National Laboratory (ORNL), Sandia National Laboratory (SNL), and the University of California at Los Angeles (UCLA). A list of collaborations at DIII-D is given in Table 1.1-3. In addition to hosting scientists and engineers to participate in DIII-D experiments, GA scientists and engineers participate on the experiments at other national and international laboratories and universities. We are active participants on the ITER and Tokamak Physics Experiment (TPX) programs and participate in the development of a physics database for the national Transport Task Force (TTF).

TABLE 1.1-3

Collaborators Participating at Dill-D

\begin{tabular}{lll}
\hline $\begin{array}{l}\text { International } \\
\text { Tokamaks }\end{array}$ & $\begin{array}{c}\text { National } \\
\text { Laboratories }\end{array}$ & Universities \\
\hline JT-60U (Japan) & LLNL & UCLA \\
JET (EC) & ORNL & UCSD \\
ASDEX (Germany) & SNLA & UC Irvine \\
TEXTOR (Germany) & SNLL & UC Berkeley \\
Tore Supra (France) & ANL & MIT \\
T-10 (Russia) & PPPL & RPI \\
TSP (Russia) & INEL & Cal Tech \\
COMPASS-D (England) & & Johns Hopkins Un. \\
TCV (Switzerland) & & N. Carolina State Un. \\
& & Univ. of Maryland \\
& & Univ. of Illinois \\
& & Univ. of New Mexico \\
& & Univ. of Paris \\
& & Univ. of Washington \\
& & Univ. of Wisconsin \\
\hline
\end{tabular}


The Fusion Energy Advisory Committee (FEAC) was charged by the DOE to develop recommendations on how best to pursue the goal of a practical magnetic fusion reactor in the context of several budget scenarios covering the period FY94-FY98. FEAC identified DIII-D as one of the high priority U.S. Fusion program elements. In its report of September 1992, FEAC noted that the DIII-D program supports both ITER and the development of an optimized tokamak. DIII-D will be the largest operating U.S. tokamak in the post-Princeton Tokamak Fusion Test Reactor (TFTR) era. The DIII-D divertor and advanced tokamak upgrades together with the strong national collaborative program will provide the U.S. with an internationally competitive magnetic fusion tokamak facility until the operation of TPX and ITER.

\subsection{HIGHLIGHTS OF THE FY92 DIII-D RESEARCH PROGRAM}

\subsubsection{DIVERTOR RADIATION}

The anticipated heat loads at the divertor plates in next-generation tokamaks are expected to be large enough to cause concern both from the standpoints of mechanical integrity and erosion rate. For the present ITER design, peak heat fluxes in the neighborhood of $20 \mathrm{MW} / \mathrm{m}^{2}$ could occur. By raising the radiated power level in the divertor, it may be possible to reduce heat peak heat flux values to more manageable levels.

Deuterium gas is injected into the vessel during the discharge to create the radiative divertor. Previous work with $D_{2}$ injection into DIII-D discharges showed significant reduction in peak heat flux at the divertor with little, if any, penalty to plasma energy confinement. In FY92, we extended our analysis of the DIII-D radiative divertor experiments, including the effects of gas injection rate and locations, input power, and separatrix $\mathrm{X}$-point height. Our analysis also points out the important role during $\mathrm{D}_{2}$ injection that neutral pressure plays.

Radiated power in the divertor during mild edge localized mode (ELM) plays a determining role in heat flux reduction, regardless of the gas puff rate, the gas puff location, and the input power. An important by-product of the DIII-D divertor studies has been the realization that neutral gas pressure in the midplane may provide the key to feedback control of the divertor process. This relationship between heat flux reduction and midplane neutral pressure is shown in Fig. 1.2-1. Our results suggest that neutral gas pressure in the midplane may provide a useful and reliable feedback parameter during $D_{2}$ injection experiments. 
Fig. 1.2-1. Peaked heat fiux is failly independent of both $D_{2}$ injection rate and injection location, when plotted versus neutral pressure in the midplane and divertor.

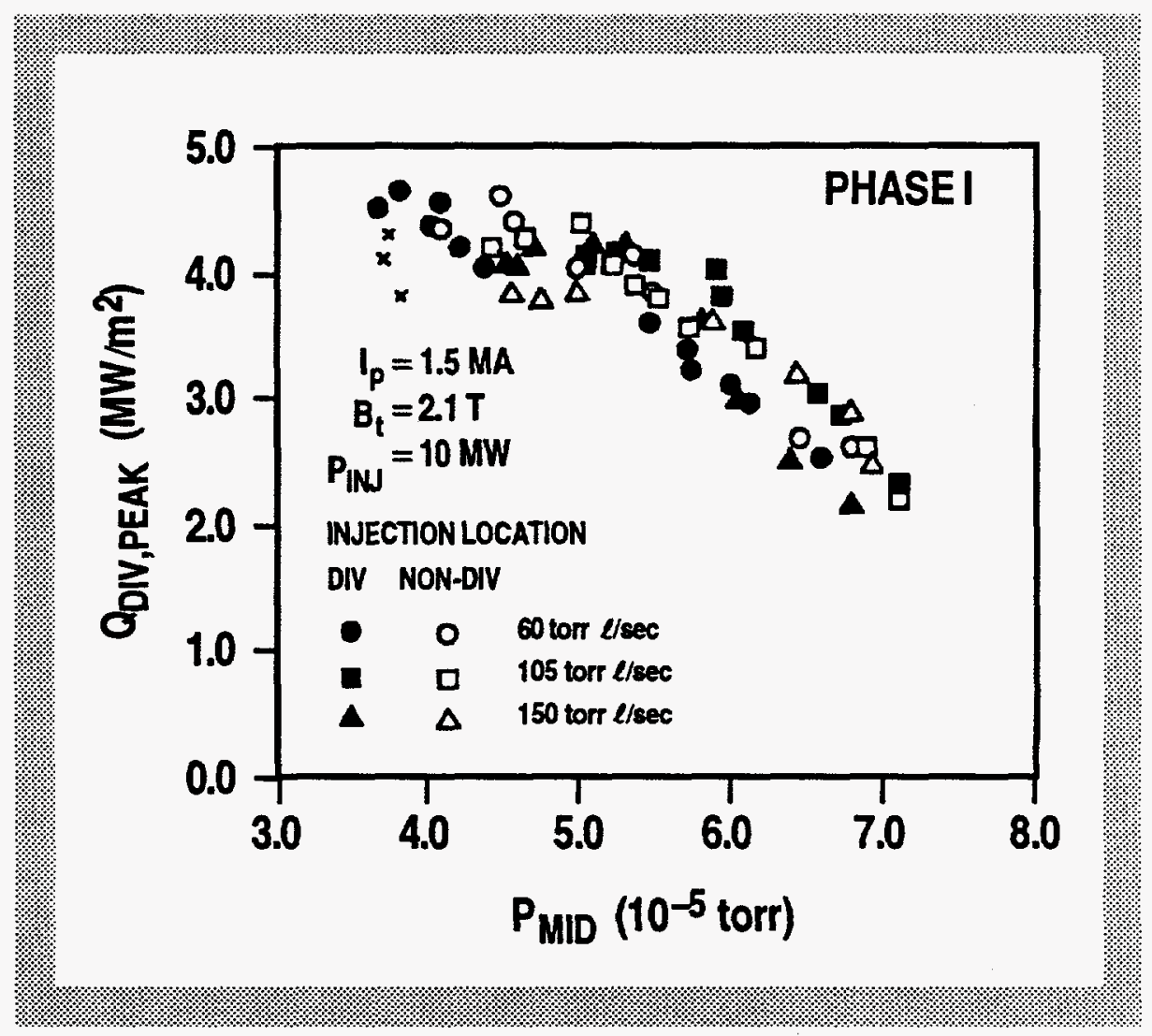

\subsubsection{IMPROVED UNDERSTANDING OF VH-MODE}

VH-mode is a regime of improved $\mathrm{H}$-mode confinement which was achieved following boronization of the DIII-D vacuum vessel. Thermal energy confinement times, $\tau_{\mathrm{E}}$, in VH-mode are up to a factor of 2 larger then values predicted by scaling from ELM-free $\mathrm{H}$-mode discharges before boronization, and up to 3.6 times values predicted by ITER-89P L-mode scaling, shown in Fig. 1.2-2. These high confinement values were obtained at high normalized toroidal beta, $\beta_{\mathrm{N}} \equiv \beta_{\mathrm{T}} /(\mathrm{l} / \mathrm{aB})>3$, and relatively high plasma currents $I \leq 2 \mathrm{MA}$. This resulted in the highest fusion plasma performance achieved to date on DIII-D, $\mathrm{n}_{\mathrm{D}}(0) \mathrm{T}_{\mathrm{i}}(0) \tau_{\mathrm{E}} \approx 2 \times 10^{20} \mathrm{~m}^{-3} \mathrm{keV}$-sec. Work in 1992 has concentrated on improving the understanding of the VH-mode confinement enhancement and on extending the duration of $\mathrm{VH}-$ mode.

The onset of the VH improved confinement is preceded by an extension of the region of high $\mathrm{E} \times \mathrm{B}$ velocity shear further in from the plasma boundary resulting in a larger region of turbulence suppression as observed by far-infrared (FIR) scattering and 
reflectometry. The region of increased $\mathrm{E} \times \mathrm{B}$ velocity shear is consistent with the region of reduced effective thermal diffusivity in VH-mode. It is also found that discharges in Fig. 1.2-2 with a higher energy confinement enhancement factor have a larger $E \times B$ velocity shear penetration.

\subsubsection{FAST WAVE CURRENT DRIVE (FWCD)}

FWCD has been selected as a backup technique for driving current noninductively near the center of the plasma of the ITER tokamak. Fast waves (FWs) may be damped directly by the electrons through the processes of electron Landau damping and transit-time magnetic pumping. When the waves are transmitted in one direction, the wave damping accelerates electrons and drives current.

A series of experiments at the end of FY91 and the beginning of FY92 with $\pi / 2$ antenna phasing demonstrated for the first time that noninductive FWCD could be obtained. Substantial

Fig. 1.2-2 Variation of energy confinement time enhancement factor with average triangularity of the separatrix flux surface for singlo- and doublenull discharges. Lowest triangularity singlonull enhancement factor may be limited by earty onset of ELMs.

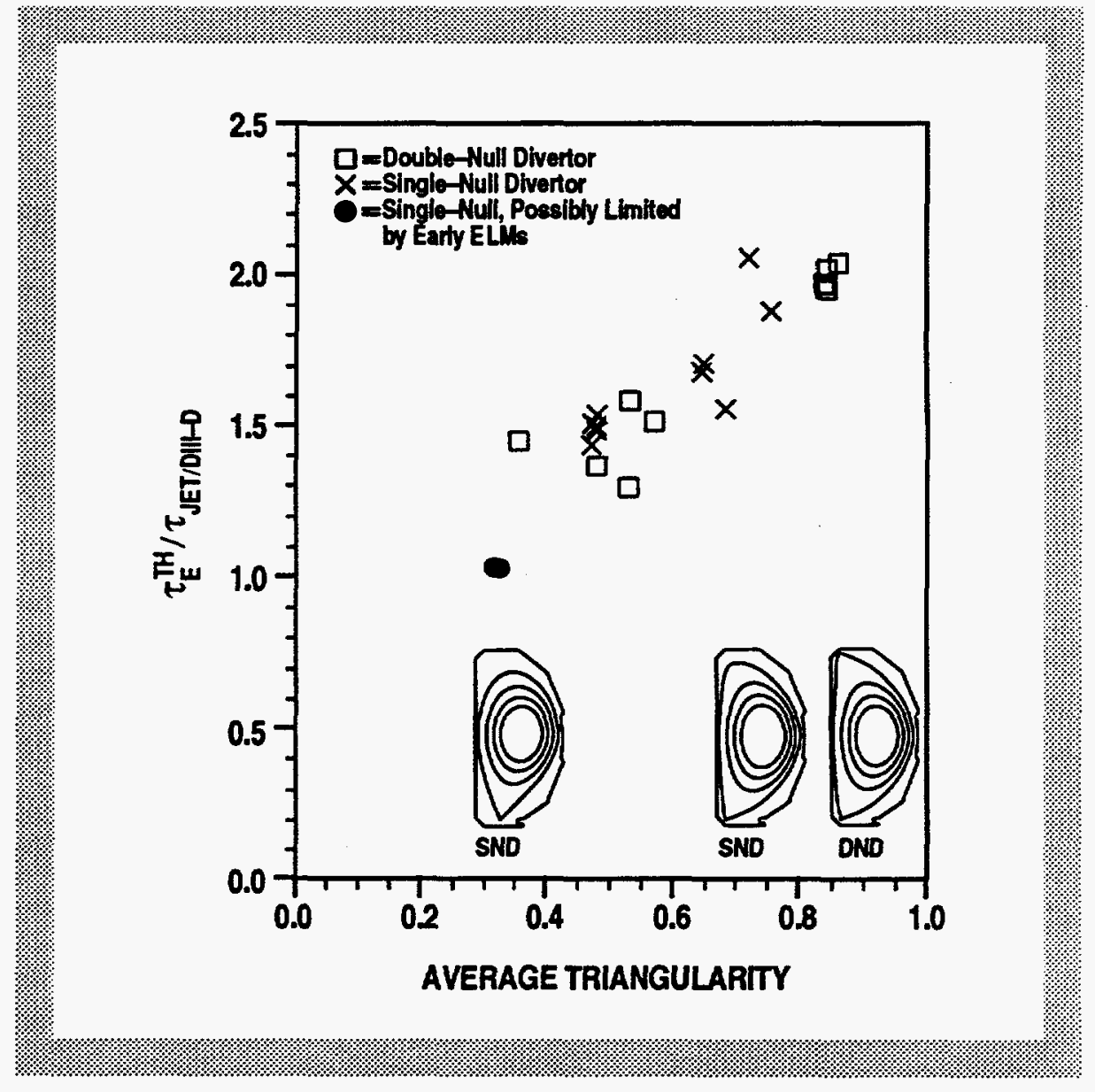


electron heating and decreases in loop voltage (the total plasma current is held constant by feedback control) were observed during FWCD at toroidal fields of 1.0 and $2.0 \mathrm{~T}$ with or without $\mathrm{ECH}$ preheating. The largest fractions of noninductively driven current were observed with $\mathrm{B}_{\mathrm{T}}=1 \mathrm{~T}$, and $\overline{\mathrm{n}}_{\mathrm{e}}=1.0 \times 10^{19} \mathrm{~m}^{-3}$ with $\mathrm{ECH}$ preheating. Up to $0.16 \mathrm{MA}$ of noninductively driven current was observed, with a total $\mathrm{I}_{\mathrm{p}}=0.40 \mathrm{MA}$.

The current drive efficiency is higher for the cases with ECH preheating than those with FW alone. This is primarily due to the higher central electron temperature obtained with preheating. Figure 1.2-3 shows that the current drive efficiency (not including the bootstrap contribution) increases with the central electron temperature. The measured efficiency is consistent with the scaling $\gamma \sim 0.4 \mathrm{~T}_{\mathrm{e}}(0) / 10 \mathrm{keV} /\left(2+\mathrm{Z}_{\mathrm{eff}}\right)$ developed for ITER. Future experiments at higher FW and electron preheating power levels should permit fully noninductive current drive in DIII-D at the 0.4 MA level at $\bar{n}_{e} \sim 1 \times 10^{19} \mathrm{~m}^{-3}$, PFW $=$ 2.0 MW, and correspondingly larger effects on the current profile.

Fig. 1.2-3. Current drive efficiency $\gamma$ for the same discharges as in Fig. $3.5-3$ as a function of central electron temperature. Also shown is the ITER Physics Design Guideline for the FWCD efficiency.

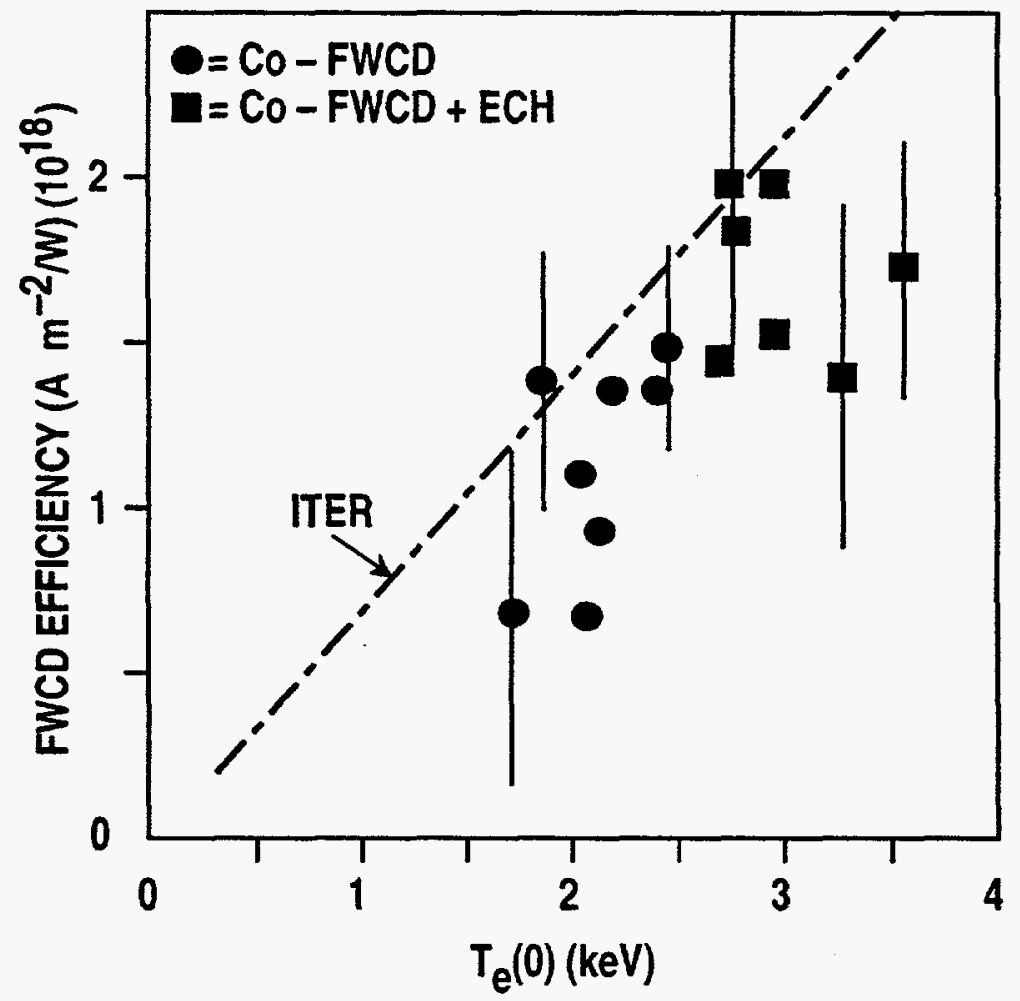




\subsubsection{TOROIDAL ALFVÈN EIGENMODES (TAE)}

The observed scaling of the toroidal Alfvèn eigenmodes (TAE) mode frequencies with the Alfvèn speed $v_{A}$ predicted by the simplest theories has now been shown to be in very good agreement with the more sophisticated predictions from ideal magnetohydrodynamic (MHD) calculations with the GATO code using the reconstructed experimental discharge equilibria. The computed and observed mode frequencies are shown in Fig. 1.2-4.

The theoretical predictions and experimental observations of TAE modes on DIII-D have been shown to be in good agreement. Also analysis of the experimental data in one discharge has now confirmed the presence of the predicted elongation-induced Alfvèn eigenmode (EAE) in DIII-D. Theoretical calculations have also predicted the existence of a new kind of global mode in finite $\beta$ discharges — the beta-induced Alfvèn eigenmode (BAE) which, in addition to contributing to the observations at moderate $\beta$, also provides an explanation of the dependence of the observed mode frequencies at high $\beta$.

Fig. 1.2-4. TAE frequency dependence on $V_{A}$. observed (solid dots) and calculated (diamonds) frequencies at increasing toroidal magnetic field. More refined analysis of the experimental data is shown as the open circles.

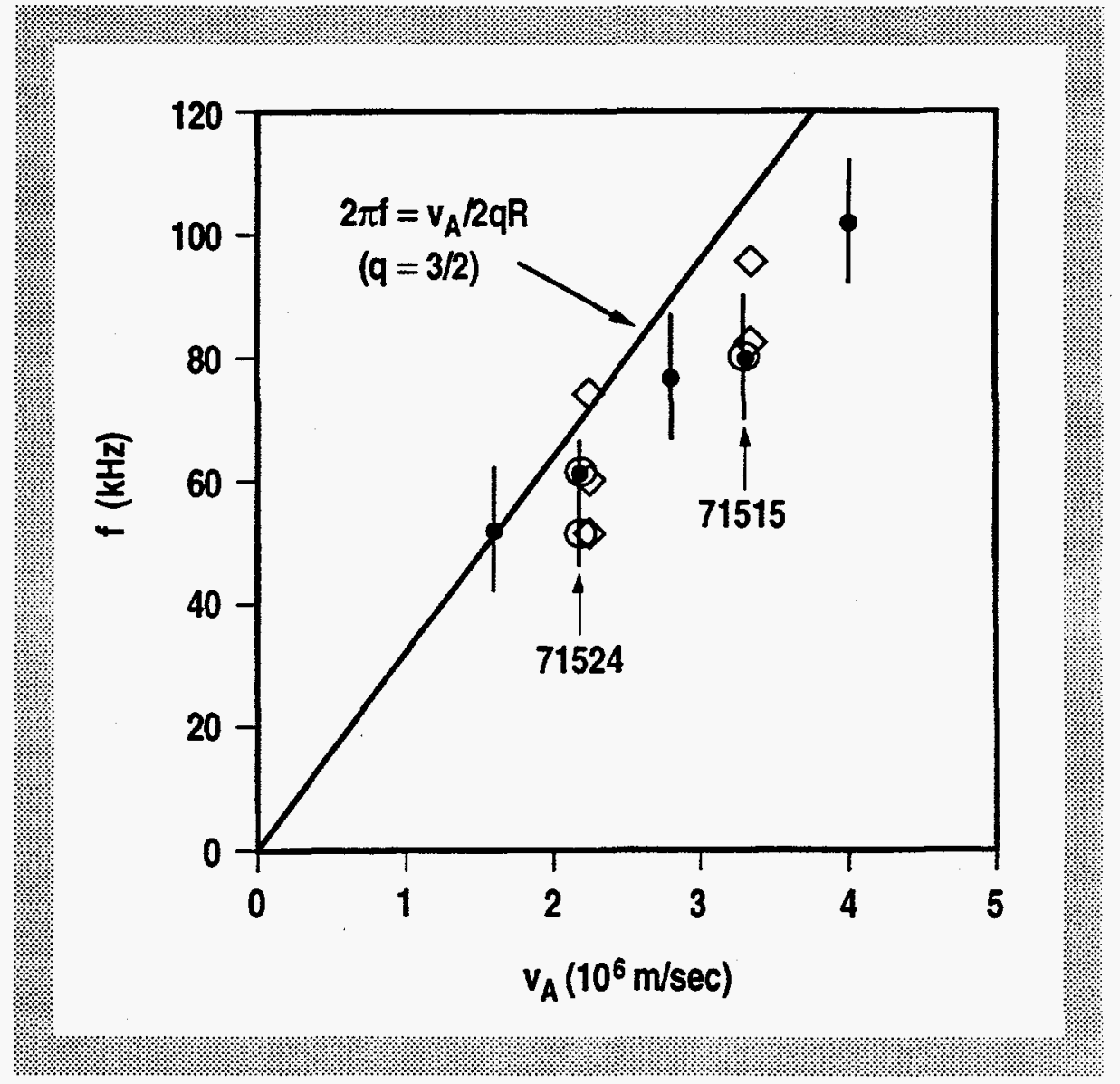





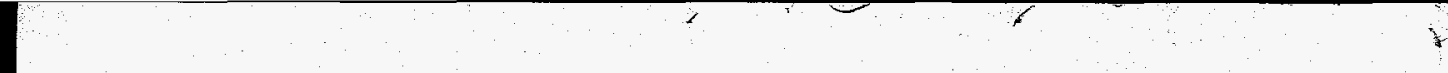




\section{DIVERTOR AND BOUNDARY RESEARCH PROGRAM}

\subsection{OVERVIEW}

The divertor and scrape-off layer (SOL) plasmas play a crucial role in establishing the overall performance capabilities of the tokamak. In addition, conditions in this boundary layer are increasingly found to be crucial in achieving the greatest performance throughout the plasma as well. Therefore, enhancing the performance and efficiency of the tokamak requires a better understanding of boundary layer physics and the development of advanced boundary layer control technologies.

The DIII-D boundary research program is designed to address key issues and concepts for the boundary plasma of future tokamaks. This work is organized into three key areas of activity. These research areas include: (1) basic divertor and edge physics including radiative divertor concepts and biased divertors; (2) development of theoretical and computer models to predict the operational parameters of future machines; and (3) impurity production, transport and control to devise boundary concepts for future tokamaks. A summary of significant findings and advancements made in each of these areas is given in this section.

Some key results in the boundary plasma research program are:

- Scaling of divertor and boundary plasma parameters and profiles.

- Measurements of fluctuation driven particle transport by the Sandia National Laboratory, Albuquerque/University of California at Los Angeles (SNLA/UCLA) fast scanning Langmuir probe.

- Modeling of the radiative divertor experiments which link heat flux reduction to input power and midplane neutral pressure.

- Imaging of divertor heat flux asymmetries during VH-mode. 
- Initial data from the neutral lithium beam edge diagnostic.

- Development of the 2-D code LEDGE to model the DIII-D boundary layer.

- Use of the DIII-D boundary layer database to study scaling of divertor and edge parameters under a variety of conditions.

- Demonstration of noninductive startup of a tokamak plasma using the advanced divertor bias ring.

- Observation of a robust SOL during VH-mode leading to improved screening of impurities.

- Improved boronization techniques allowing for a lower power threshold for VH-mode.

\subsection{DIVERTOR PHYSICS}

The divertor physics group activities are designed to provide timely input for critical International Thermonuclear Experimental Reactor (ITER) design issues while allowing opportunities to explore options, which appear to be attractive for increasing the capabilities of diverted tokamaks. In 1992, significant progress was made in characterizing and understanding the SOL and divertor plasmas under a variety of conditions. Radiative divertor studies have begun to uncover the important processes for dissipating heat flux through radiation. In addition, innovative concepts using the advanced divertor hardware have been tested. The DIII-D divertor program has begun to produce results that are needed to design innovative, workable divertors for future reactor tokamaks.

\subsubsection{DIVERTOR AND SOL SCALING STUDIES}

The goal of these studies is that of finding scaling relations between the SOL and main discharge parameters. Such information can be used directly in tokamak design as well as to check the accuracy of predictive models such as the Braams B2, LEDGE, or NEWT-1D codes. Substantial progress was made in 1992 with a number of scaling studies. Though our understanding is incomplete, a number of important relationships were observed experimentally. 
GA-A21186; FY92 DIII-D Research Operations Annual Report

The peak divertor heat flux is found to scale linearly with input power, as shown in Fig. 2.2-1(a). Some models predict the peak heat flux to rise faster than linear. This scaling indicates that the heat flux is limited by sheath conditions in the divertor, though other factors may play a role. The separatrix density is also found to rise with input power from more than $30 \%$ of the average density to as much as $60 \%$. Most models have assumed the separatrix density to be a constant $30 \%$ of the average density. This encouraging result should help to reduce the high heat flux and electron temperatures at the divertor plate for the highest input powers.

The scaling of peak heat flux with toroidal field and plasma current, shown in Figs. 2.2-1(b) and 2.2-1(c), has also been examined. There is a weak dependence with toroidal field strength, which is consistent with that expected from the change in parallel connection length. There is a strong increase of peak heat flux with plasma current, however. This dependence suggests that the perpendicular energy transport is reduced as the current increases. Assuming transport scaling as $\chi_{\perp} \propto \mathrm{Ip}$, this scaling relation was predicted by the NEWT-1D code.

Measurements with the divertor Langmuir probes have provided some data on the scaling of plasma parameters at the divertor plate with input power and neutral gas pressure in the divertor. The plasma temperature at the divertor is important because of its effect on sputtering of the divertor targets. The electron temperature at the outer strike point increased linearly (Fig. 2.2-2) with input power from 7 to $45 \mathrm{eV}$ as the beam power increased from 2 to $14 \mathrm{MW}$. The divertor plasma density also increased from $2 \times 10^{20} \mathrm{~m}^{-3}$ to $4.5 \times 10^{20} \mathrm{~cm}^{-3}$ over this power range, Fig. 2.2-2. This result is encouraging because some models predict that the divertor electron temperature would increase as $\mathrm{P}^{2}$ inj. The electron temperature was also found to decrease at higher neutral gas pressure. At $6.5 \mathrm{MW}$ of beam power, the electron temperature at the outer strike point decreased from 52 to $23 \mathrm{eV}$ as the neutral pressure in the divertor increased from 0.4 to $3.1 \mathrm{mtorr}$. The central density increased by $25 \%$ over this range, but the core energy confinement did not change. This is further evidence that the divertor conditions can be managed to tolerable levels with the use of neutral gas.

The $\mathrm{X}$-point height is an important consideration in designing a divertor. A high $X$-point, and thus large divertor, may reduce the peak electron temperature at the divertor plates, but requires a costly increase in machine size. Experimental results show that 


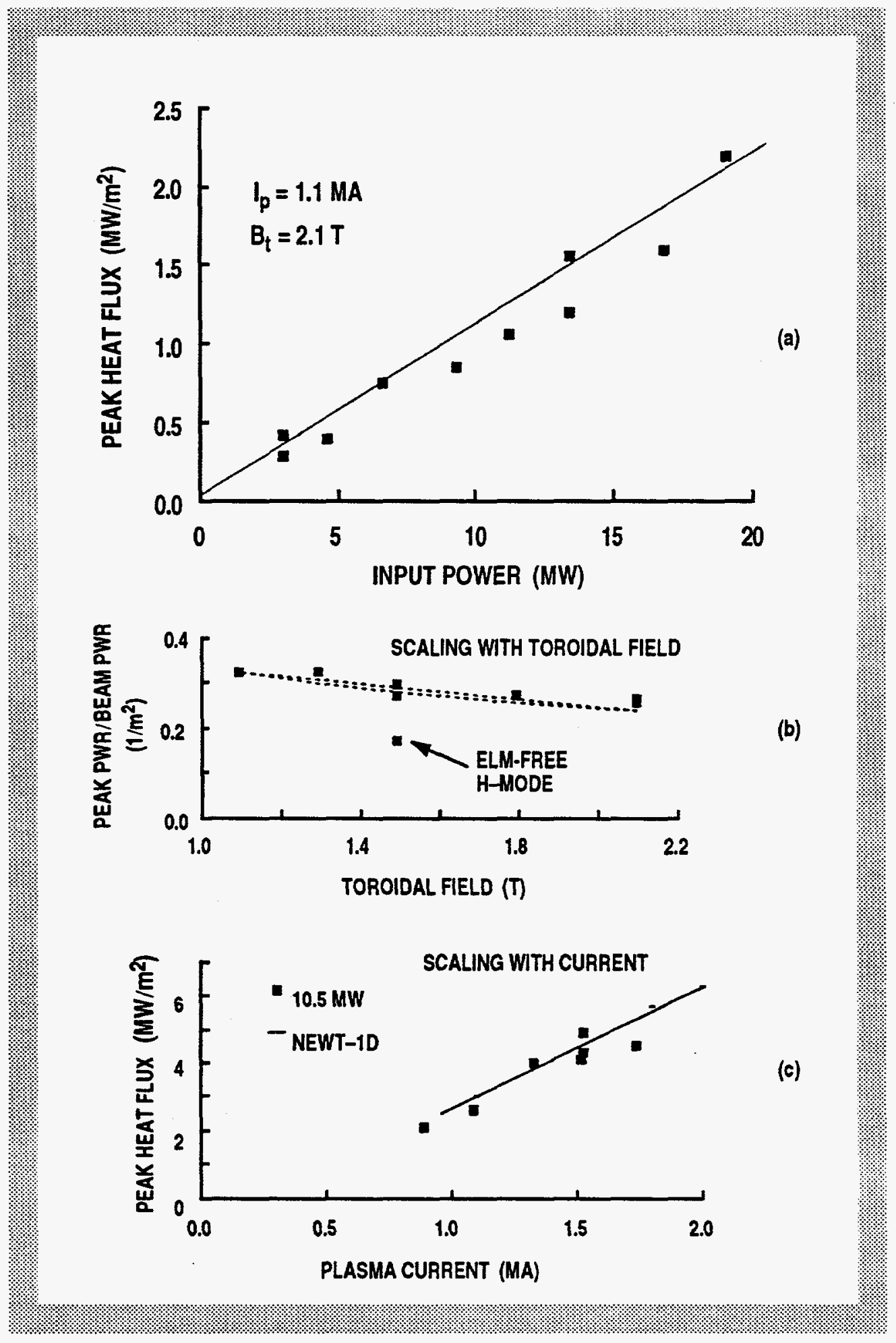

Fig. 2.2-1. Scaling of the peak heat flux to the divertor for ELMing H-mode plasmas. (a) Peak heat flux versus input power. (b) Peak heat flux, normalized to input power, versus toroidal field. (c) Peak heat flux versus plasma current. 
decreasing the distance from the $\mathrm{X}$-point to the target plates does produce a modest increase in electron temperature at the divertor. However, there does not appear to be a corresponding increase in core plasma impurity content until the $\mathrm{X}$-point is nearly touching the target plates. This is consistent with previous results showing that the walls are the major impurity source. These $\mathrm{X}$-point height results indicate that a divertor of modest size may be sufficient for future tokamaks.

The effect of edge localized modes (ELMs) are also important as future high-power long pulse tokamaks such as ITER are expected to operate with $\mathrm{H}$-mode and ELMs. ELMs in DIII-D were found to expel more particles than energy, and this has a very beneficial effect on the divertor conditions, i.e., a lower electron temperature at the divertor. The particle loss rate from ELMs is sufficient to offset the density rise observed in ELM-free H-mode. Though the peak heat flux during an ELM is 50 times higher than the average heat flux, this burst lasts less than $500 \mathrm{msec}$. The fact that the rapid ELMs do not directly carry much of the energy out of the plasma means that they are not a very important source of divertor heating, so designers of future tokamaks should focus on handling the steady heat flux that is observed all during $\mathrm{H}$-mode.

Fig. 2.2-2. Electron temperature and electron density of the outer strike point versus input power.

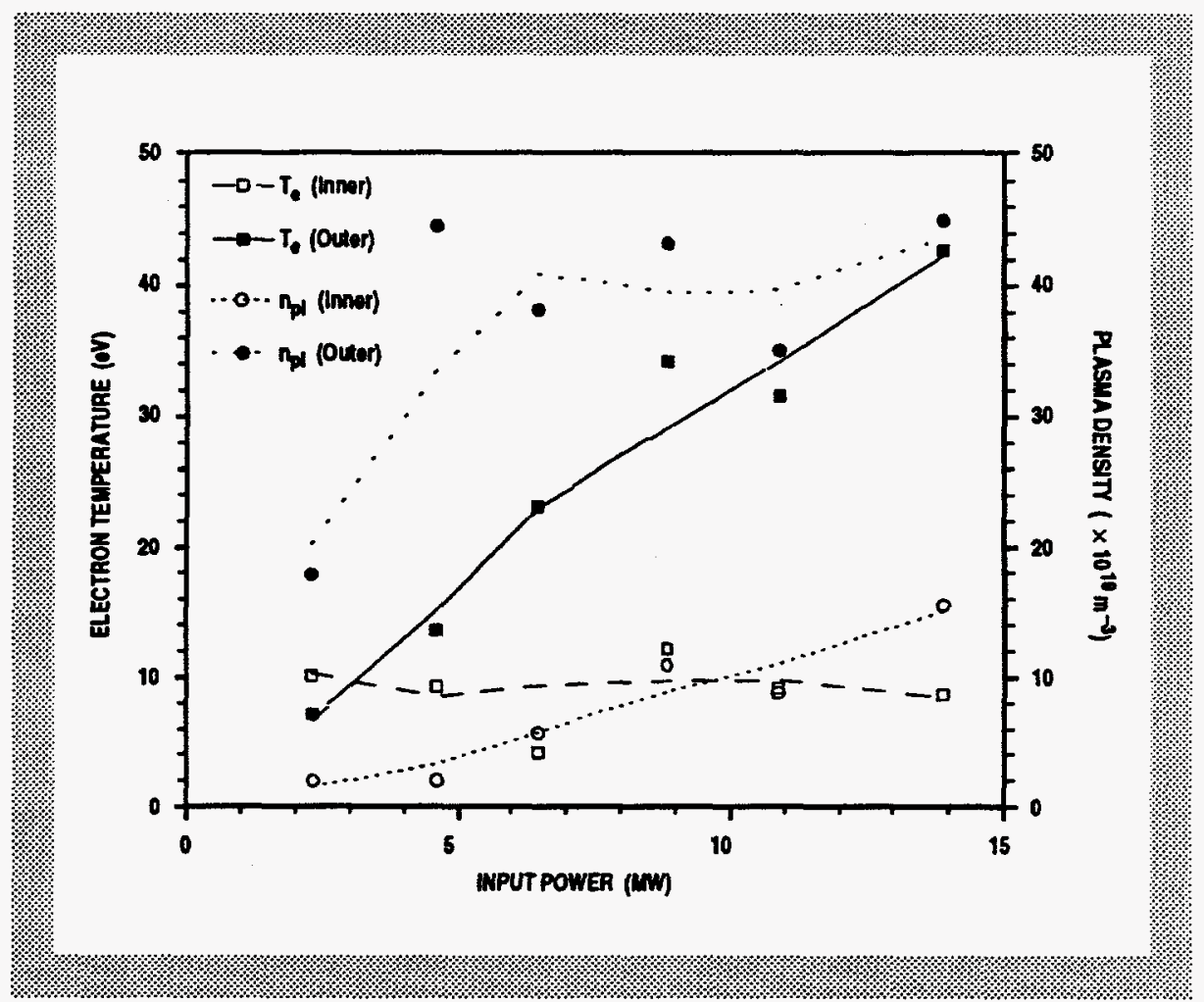




\subsubsection{SCRAPE-OFF LAYER MEASUREMENTS}

The SNLA/UCLA fast reciprocating Langmuir probe, mounted on the outboard midplane of the DIII-D tokamak, has been used to make direct measurements of the SOL. These measurements have provided information on the scaling of boundary plasma profiles, divertor physics, transport mechanisms in the boundary plasma, $\mathrm{L}-\mathrm{H}$ transition physics, and the effects of boundary plasma biasing with the advanced divertor bias ring. Work this past year has concentrated on measuring the SOL width under a variety of conditions and measuring the fluctuation-induced particle transport.

Outer SOL plasma profiles and turbulent transport have been measured in $\mathrm{L}-, \mathrm{H}-$, and VH-mode discharges with lower single-null and double-null divertors. In VH-mode, the SOL plasma is usually hotter, denser, and has a faster spatial decay than in $\mathrm{H}$-mode. Typical density e-folding lengths, $\lambda_{\mathrm{n}}$, shown in Fig. 2.2-3, for $\mathrm{L}-\mathrm{H}-$, and VH-modes respectively are: $2.6,1.3$, and $0.75 \mathrm{~cm}$.

Boundary plasma scaling studies have been performed by varying $B_{t}, I_{p}$, and $q_{95}$ (connection length $L_{\|}$to the divertor target) and measuring the density within the

Fig. 2.2-3. Density profiles in the SOL for $L-, H-$, and VHmodes, measured by the SNLANCLA probe.

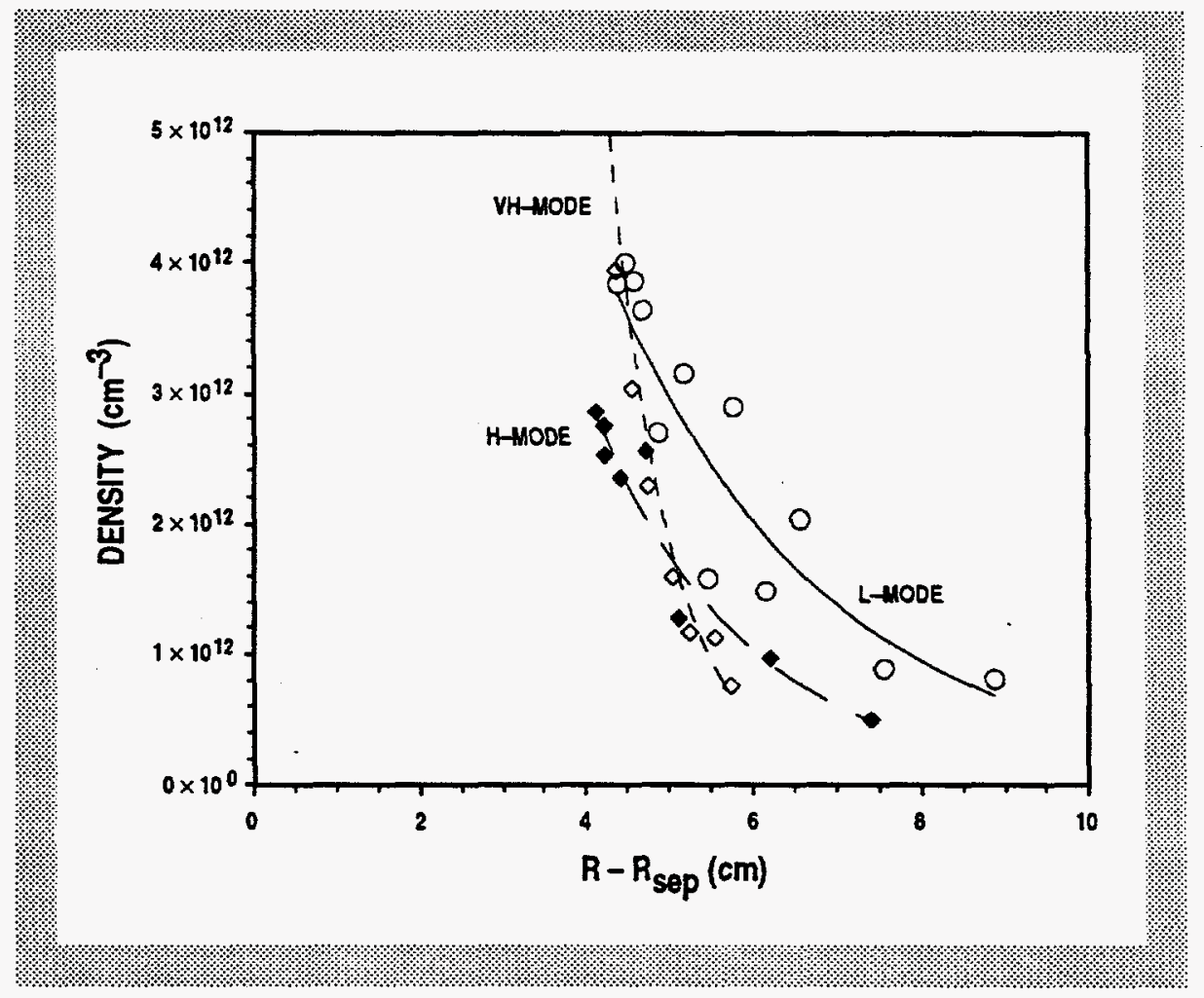


SOL. These parameter scans have produced the following scalings: $\lambda_{n} \propto\left(\mathrm{q}_{95}\right) \alpha$, where $\alpha=1.2-1.5, \lambda_{n} \propto\left(1 / I_{p}\right) \alpha$, where $\alpha(L-m o d e)=1.5$, and $\lambda_{n} \propto\left(B_{t}\right) \alpha$, where $\alpha$ $(\mathrm{L}-$ mode $)=1.25$.

The SNLA/UCLA probe can also be used to directly determine particle flux by measuring density and potential fluctuations and their respective phases. Preliminary efforts to compare the scalings in either $\mathrm{L}$ - or $\mathrm{H}$-mode with variations in the measured microturbulence-driven transport have, so far, been unsuccessful. Over the extent of the $I_{p}$ and $B_{t}$ scans, the fluctuation data predicts only a change in transport of $20 \%$ to $30 \%$, while the measured SOL widths require changes by a factor of $2-3$. We are continuing to investigate the scaling of the microturbulence and the dc convective particle transport in an effort to determine if the observed scalings arise primarily due to changes in the fluctuation driven transport.

Across the $\mathrm{L}-\mathrm{H}$ transition, $\lambda_{\mathrm{n}}$ is measured to decrease by a factor $1.5-2$, implying a reduction in the effective perpendicular diffusion coefficient $D_{\perp}$ of at least 3-4. In this case, the measured turbulent particle transport is reduced abruptly at the $\mathrm{L}-\mathrm{H}$ transition by a factor of order 8-16, as shown in Fig. 2.2-4. The measured $\lambda_{n} s$ and particle fluxes imply a reduction in $D_{\perp}$ of an order-of-magnitude. Limited modeling of the Thomson density and electron temperature profiles in similar L- and $\mathrm{H}$-mode discharges with the LEDGE code requires similar order-of-magnitude reductions in $\mathrm{D}_{\perp}$. Typical $\mathrm{L}$-mode $\mathrm{D}_{\perp}$ estimated from the turbulent particle flux are $0.6-0.7 \mathrm{~m}^{2} / \mathrm{sec}$; typical $\mathrm{H}-$ mode $D_{\perp}$ are $0.04-0.06 \mathrm{~m}^{2} / \mathrm{sec}$. These values are quantitatively consistent with results of LEDGE modeling of similar discharges.

\subsubsection{RADIATIVE DIVERTOR}

DIII-D has a vigorous program to study the radiative divertor concept as a method to reduce and control heat flux to the divertor target. We have made significant progress toward both categorizing and understanding the dynamics of heat reduction occurring during deuterium injection into ELMing H-mode discharges. Although DIII-D at present has an "open" divertor configuration, the enhancement in radiated power from the divertor region was found to play the major role in the observed heat flux reduction, regardless of the gas puff rate, the gas puff location, or the input power. We have found the maximum reduction in peak heat flux in the divertor (while the plasma on the 
Fig. 2.2-4. Particle transport in the SOL as measured by the SNLANUCLA probe. Particle flux drops markedly after the L-to-H transition. The radial position is measured with respect to the separatrix.

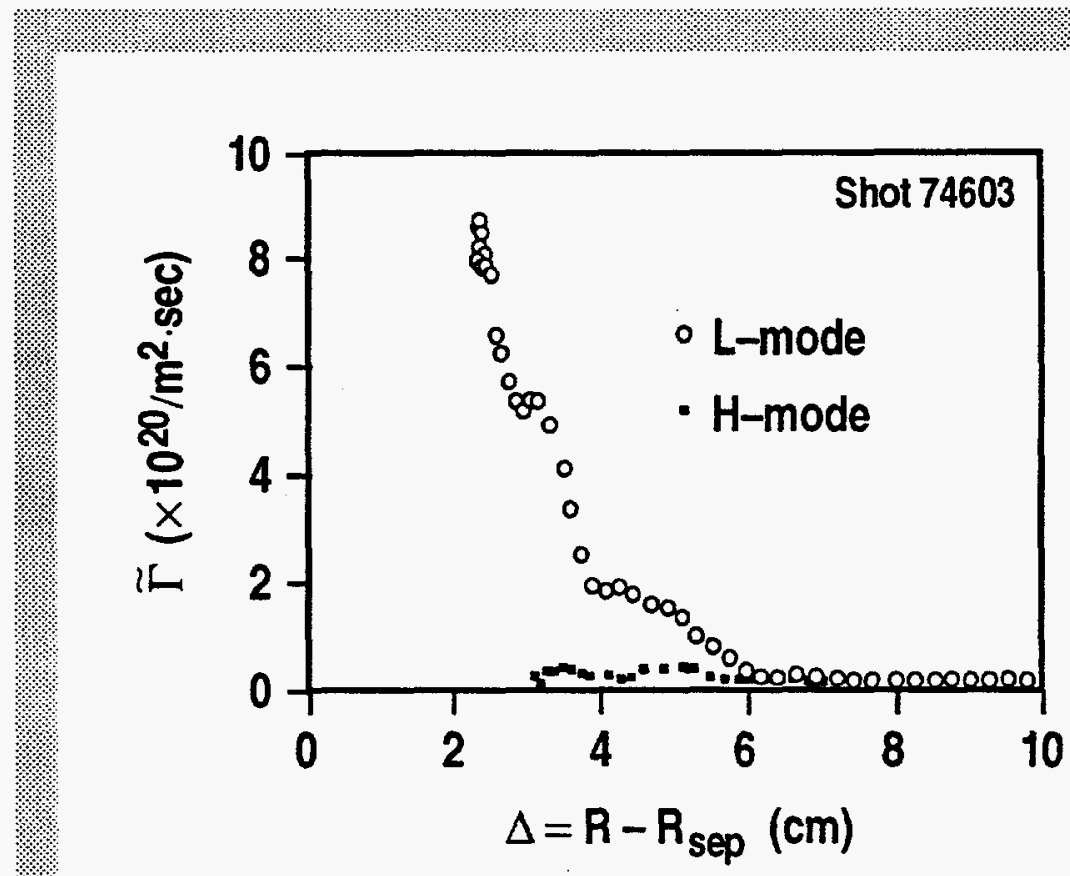

separatrix is "attached" to the divertor tiles) can be expressed in terms of neutral pressure, input power, and electron temperature. A simple model has been proposed to explain these results. An important by-product of the DIII-D radiative divertor studies has been the realization that neutral gas pressure in the midplane may provide the key to feedback control of the radiative divertor process. This relationship between heat flux reduction and midplane neutral pressure is shown in Fig. 2.2-5.

\subsubsection{HEAT FLUX PROFILE STRUCTURES AND TOROIDAL ASYMMETRIES}

Radial heat flux profiles measured on the divertor target plates often have multiple peaks. These features were explicitly studied over the last year in order to classify their characteristics and determine their cause. Since multiply peaked (i.e., bifurcated) heat flux profiles appear to be related to toroidal and poloidal asymmetries, a model used to explain nonaxisymmetric energy deposition patterns in the ASDEX divertors is being applied to this DIII-D data. In the model, small random magnetic perturbations (an intrinsic property of realistic tokamak designs) create stationary helical islands which, in some cases, cause a stochastic layer near the primary separatrix. These islands cause helical modulations in the outward heat flux as it passes through the boundary layer and produce bifurcations in the 
Fig. 2.2-5. Peak heat fiux to the divertor is reduced as the midplane pressure increases.

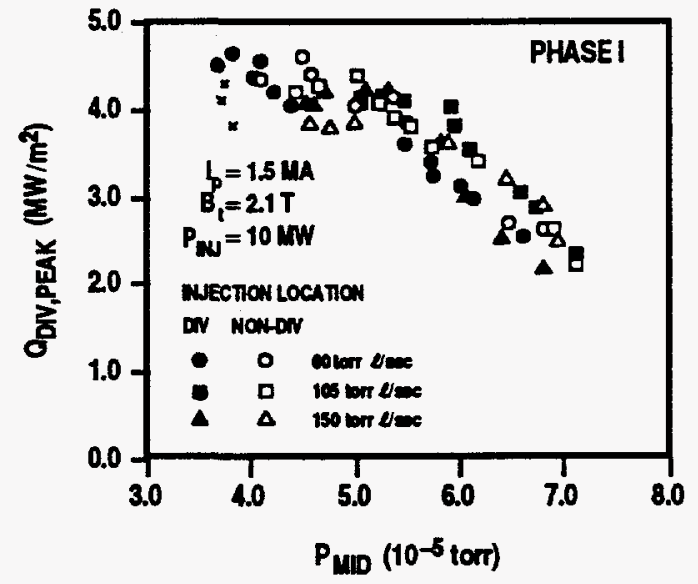

- Qmy,pak $1 \mathrm{~S}$ $-55 \%-65 \%$ of TS WITAL VALUE BY THE END OF PHASE

- MUECTOR LOCATIOI

+ DV $\rightarrow$ DIRECTLY MTO PRIYATE FLUX RECION

NON-DN $\rightarrow$ FROITHE TOP OF THE VESSEL

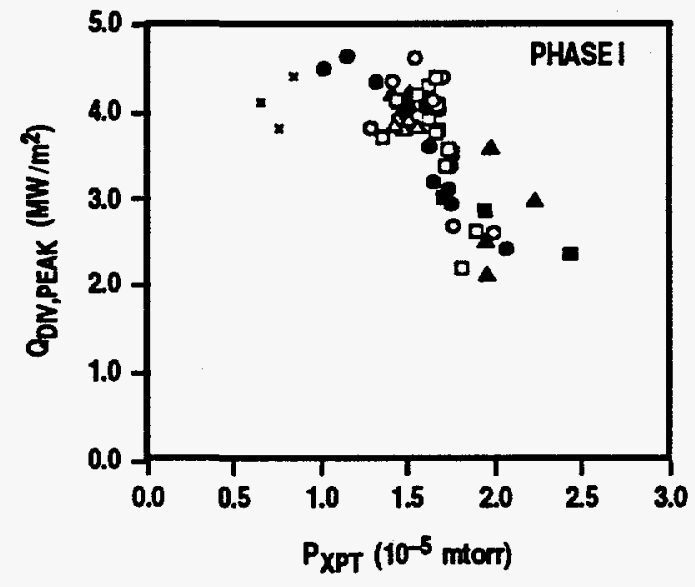

radial profile measured with infrared (IR) cameras. As with the ASDEX analysis, some conditions yield data which are well correlated with the model while others suggest that additional effects, such as a self-consistent plasma response to the magnetic islands, may be important.

Bifurcations are also observed without locked modes in VH- and L-modes. They appear more frequently in double-null divertor configuration than in single null and they are not always stationary. One particularly interesting observation, made during VH-mode operations, is that the heat flux profile on the lower divertor tiles starts as a single, welldefined peak near the strike point. As time progresses, the exponential wing of the single peak profile develops several new peaks which grow throughout the remaining part of the VH-mode. When a secondary peak reaches the amplitude of the original peak, the discharge 
drops into an $\mathrm{H}$-mode. This behavior is shown in Fig. 2.2-6 for several time slices during a double-null VH-mode shot. Future studies of these effects will be greatly enhanced by the addition of several new diagnostics made during 1992. A toroidal tile current array measuring SOL currents and a second lower divertor IR camera will be particularly important for our continuing studies of these toroidal asymmetries.

\subsubsection{LITHIUM BEAM EDGE DIAGNOSTIC}

Edge diagnostics on DIII-D have continued to be added and upgraded in order to obtain more detailed information from the boundary layer and divertor. During this past year, the lithium beam diagnostic was commissioned on DIII-D and began yielding useful information. The lithium beam diagnostic is designed to yield precise local information about the plasma edge density profile and its associated fluctuations by injecting an atomic lithium beam of ( $5-30 \mathrm{keV})$ energy and observing the plasma-induced collisional fluorescence with a multichord detector. We developed remote control and monitoring of the ion source operation and beam formation and focusing and integrated the control system and data acquisition into the DIII-D operating system. Beam-gas measurements were conducted to analyze the intrinsic beam fluctuations and stability. Fluorescence was obtained for a number of tokamak discharges.

Analysis of the initial lithium beam data has already yielded some interesting features. These include changes in the edge plasma density behavior during the L-to- $\mathrm{H}$ transition, disruptions, and ELMs. Design and construction of the full 16-channel detection system will be completed and ready for experiments beginning next year.

The discussion of other edge diagnostics is in Section 5.5.2.

\subsection{MODELING AND EDGE DATABASE}

\subsubsection{SOL MODELING}

A fully implicit 2-D fluid code (LEDGE) for analysis of the boundary plasma in DIII-D has been developed by Lawrence Livermore National Laboratory (LLNL). This code was developed from the much-used B2 code and contains much of the same physics. In addition to the more robust fully implicit numerical scheme, the code has added the following features: (1) a two-group diffusive model to simulate the existence 
GA-A21186; FY92 DIII-D Research Operations Annual Report

Fig. 22-6. Evolution of the DIIIO divertor target plate heat flux profile during a double-null VH-mode. Once the profile bifurcates and the amplitude of the secondary peak reaches that of the primary poak at 2600 msoc, a global MHD event is triggered and the VH-mode is lost.
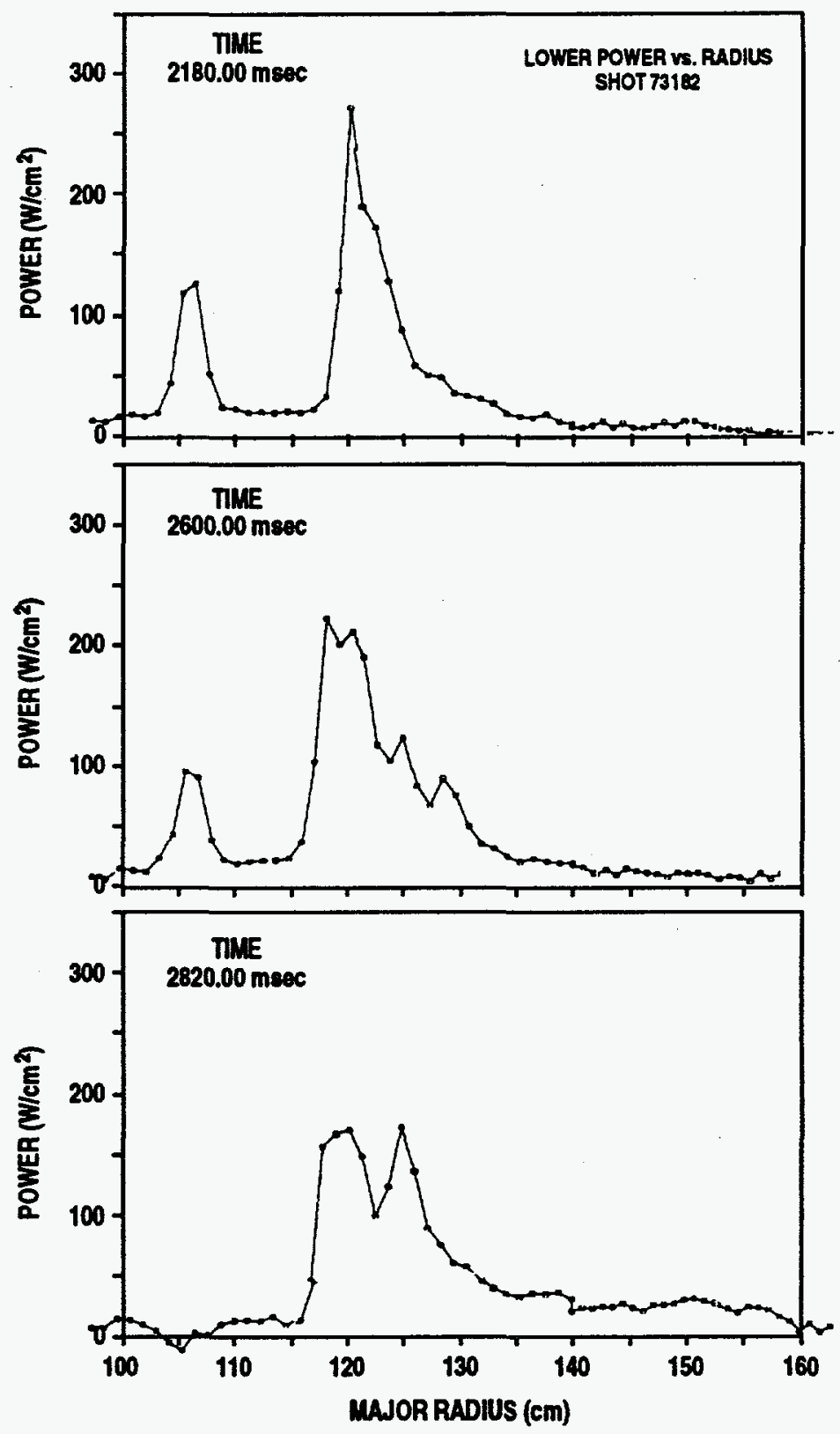

of a fast (charge-exchange) and slow (Franck-Condon) neutral species; (2) a grid generation scheme which utilizes the equilibrium geometry obtained from the EFIT code, permitting easy analysis of DIII-D experimental data; (3) the ability to simulate gas puffing and/or pumping in an arbitrary geometry around the periphery of the plasma; (4) the ability to 
include self-consistent plasma potential and the drifts associated with the resulting electric fields and pressure and temperature gradients; and (5) radiation losses from noncoronal equilibrium radiation of a fixed impurity fraction.

We have applied the LEDGE code to analysis of boundary plasma data obtained from divertor characterization experiments on DIII-D. The purpose of this analysis is to determine the scaling of perpendicular transport coefficients in the boundary plasma. It is assumed that this transport determines the width of the boundary plasma and, hence, the profile of exhaust power at the divertor floor. In this analysis, the particle diffusivity $\left(\mathrm{D}_{\perp}\right)$ and the electron and ion thermal diffusivities, $\chi_{\mathrm{e}}$ and $\chi_{\mathrm{i}}$, are varied to obtain consistency between the calculated density and temperature profiles and the measured profiles. Typical fits we obtain are shown in Fig. 2.3-1(a). In general, we find the transport coefficients to be significantly smaller than the $1-4 \mathrm{~m}^{2}$ assumed in ITER studies.

These studies have identified two radial regions in the plasma outside the last closed flux surface, as shown in Fig. 2.3-1(b). The first, a very narrow region adjacent to the separatrix, is dominated by parallel electron thermal conduction. The power carried across the separatrix by the electrons is rapidly conducted to the divertor floor in this region. This produces the peak power on the divertor. The power carried across the separatrix by the ions cannot be exhausted as rapidly as that carried by the electrons leading to a second radial region. This region has a longer radial scale length, and the power flow is determined by the ions with the electron temperature determined by electron-ion energy exchange.

The edge physics database, BNDY, contains data for the plasma core, the plasma divertor region, and the SOL for the DIII-D tokamak. It has been used to facilitate the analysis of data from experiments which were designed to study the plasma processes and energy and particle flow in the edge and divertor regions of DIII-D.

\subsubsection{EDGE DATABASE}

Initial results using the database indicate that in DIII-D, the ratio of separatrix density to average core density does not agree with the value currently assumed in modeling the ITER. The DIII-D separatrix density [as shown in Fig. 2.3-2(a)] is generally larger than the approximately $30 \%$ of the average core density assumed in ITER modeling. 
Fig. 2.3-1. Radial profile of (a) electron density, and (b) electron and ion temperature. The solid lines are calculated from the LEDGE code using $D_{1}=0.25 \mathrm{~m}^{2} / \mathrm{sec}$ and $c_{e}=c_{i}=0.5 \mathrm{~m}^{2} / \mathrm{sec}$. The ion temperature data points are measured with the CER diagnostic and the electron density and temperature data are measured with the Thomson diagnostic.
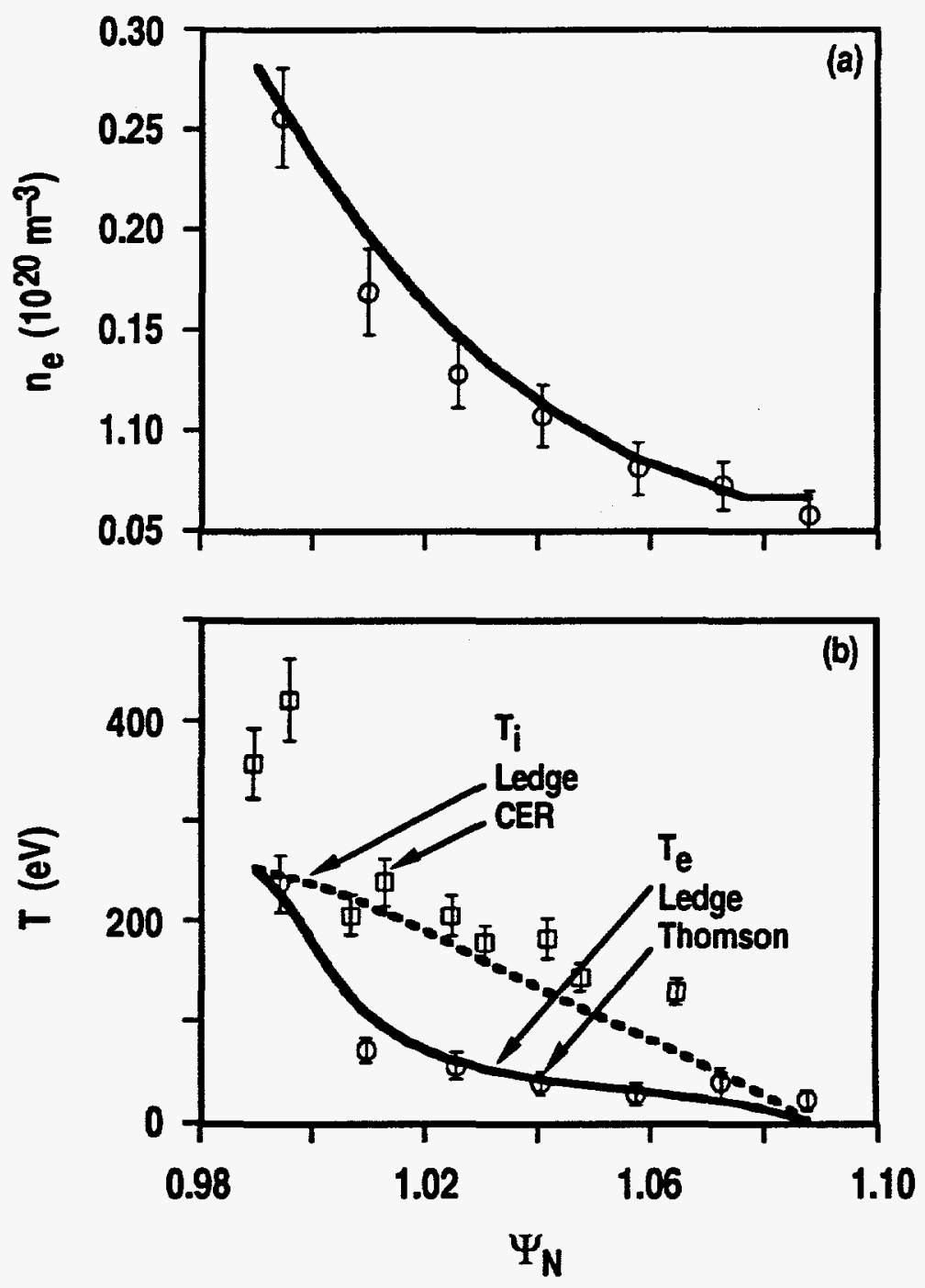

Using the database, we have analyzed the data for the measurements of the heat flux to the divertor tiles. The analysis indicates that the peak heat flux to the outer divertor displays an unexpected dependence on the inner gap distance, Fig. 2.3-2(b) (distance from the inner midplane of the plasma separatrix to the inner post), for gap distances that are much larger than the expected width of the SOL. This suggests that there may be other, as yet unknown, hidden variables that are affecting the heat flux to the divertor plates. 
Fig. 2.3-2. Scaling relationships are extracted from the boundary database BNDY. (a) The separatrix density normalized to the average density versus the separatrix pressure, $n_{e} T_{e}$, is greater than normally assumed for ITER modeling. (b) The peak divertor heat flux versus the inner gap, between separatrix and inner wall, displays an unexpected dependence.

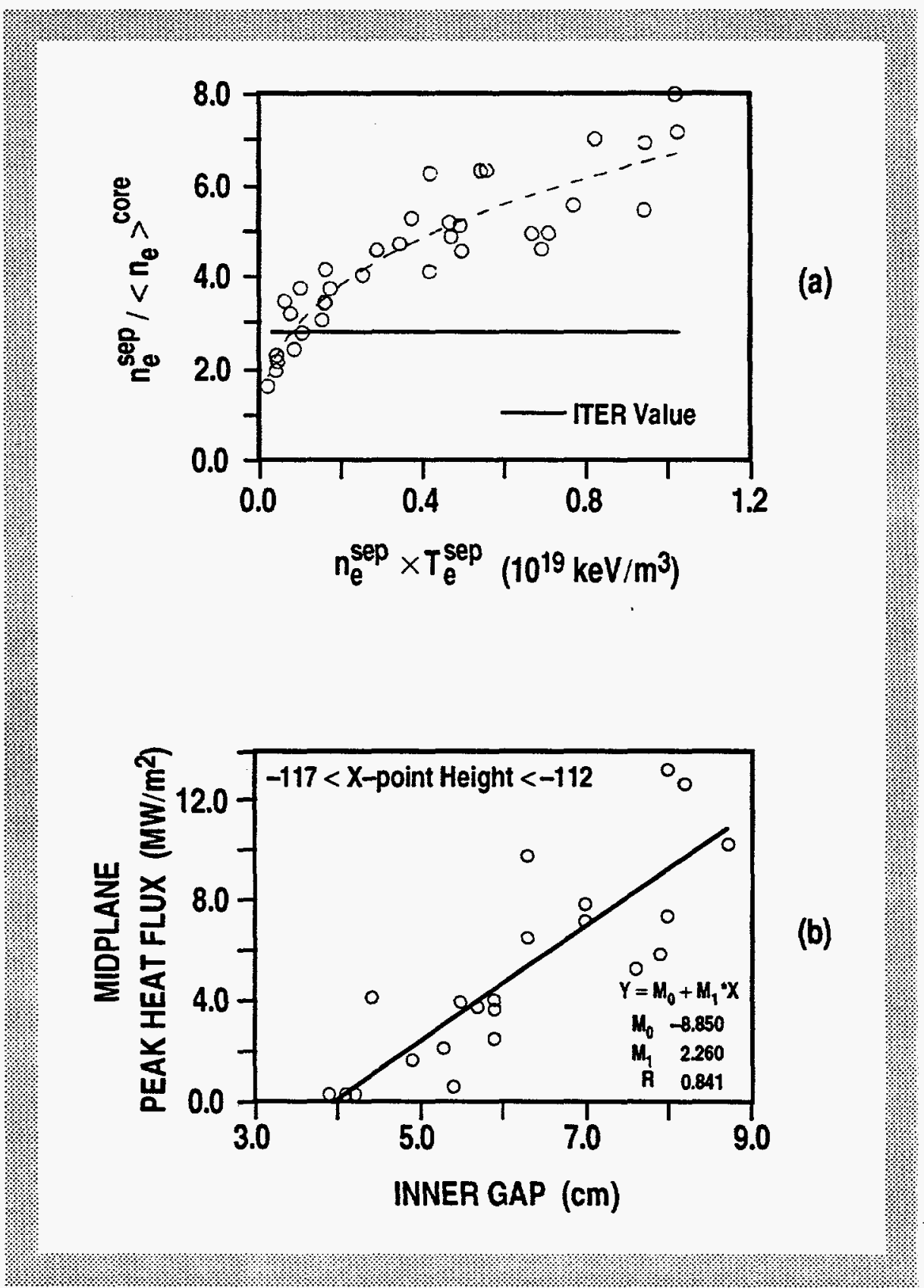

(a)

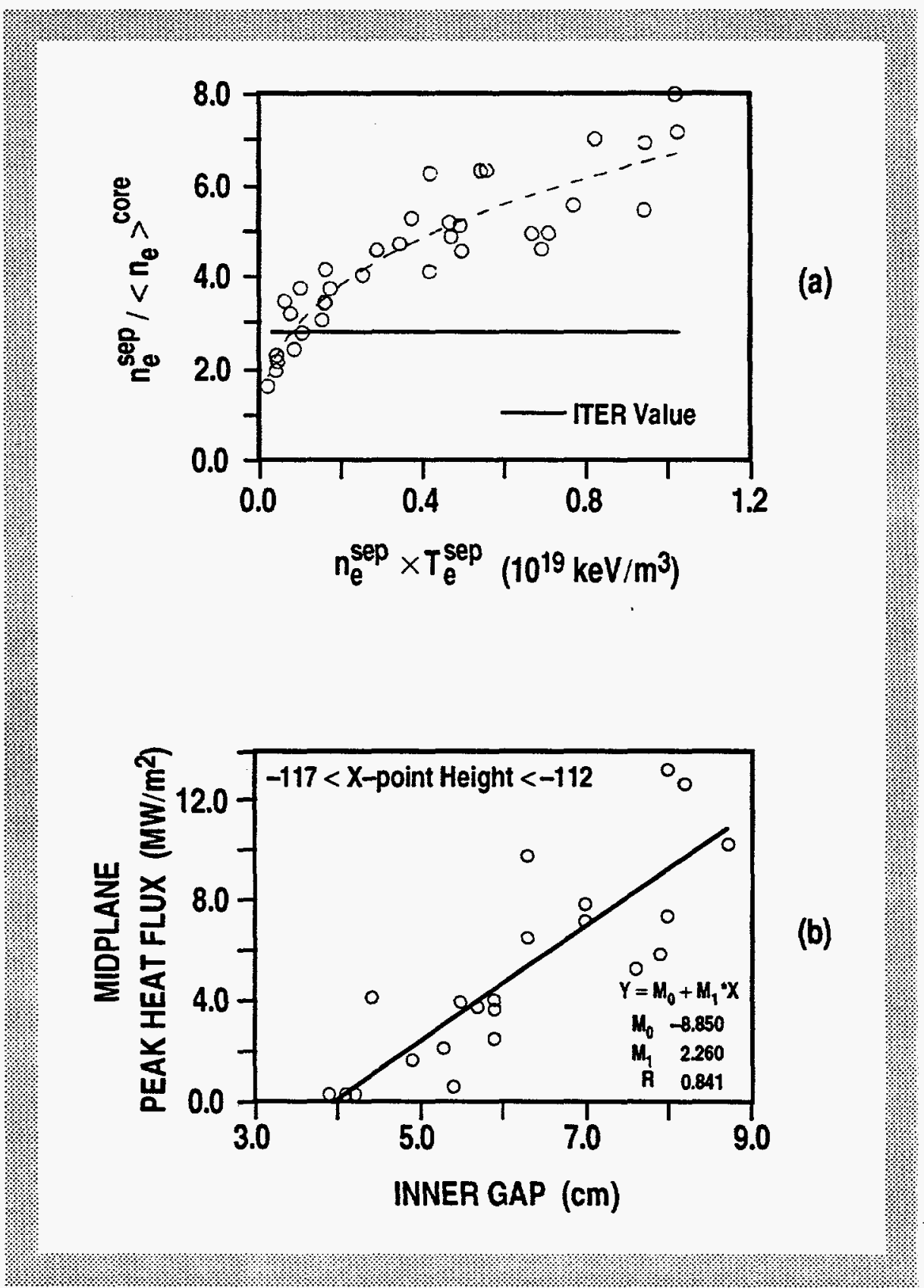

(b)

\subsection{IMPURITY TRANSPORT AND CONTROL}

During 1992, significant progress has been made in the understanding of impurity generation and transport, as well as the development of impurity control techniques to improve tokamak performance. Work this past year includes: studies of improved impurity shielding observed during $\mathrm{VH}$-mode, use of boronization for reducing impurity 
generation and improving high confinement regimes, development of a multichannel divertor spectrometer to study generation of impurities in the divertor, and helium transport studies to understand how tokamak reactors can deal with helium ash. The helium transport studies are part of a collaboration between General Atomics (GA) and Oak Ridge National Laboratory (ORNL). The results from these studies are presented in Section 10.1.2.from ORNL describing the collaboration.

During the VH-mode phase of DIII-D discharges, the impurity radiation is very low compared to standard ELM-free H-mode and does not increase in time, as shown in Fig. 2.4-1. After termination of VH-mode and the development of a more standard ELM-free $\mathrm{H}$-mode, impurity radiation increases dramatically. Early analysis of some of these discharges indicate that these VH-modes exhibit a more dense and hotter SOL than the ordinary $\mathrm{H}$-mode. This implies that the reduction in impurity content and radiation is due to shielding of the impurity atoms sputtered from the wall. An estimate of the effectiveness of the SOL in shielding the plasma from the sputtered impurities was produced by using the EFIT magnetic field mapping routine and the edge channels of the Thomson scattering diagnostic to produce a two-dimensional map of the density and temperature in the SOL, assuming $n_{e}$ and $T_{e}$ are constant on a flux surface. The net particle penetration fraction, i.e., the fraction of neutrals that penetrate through the last closed flux surface, is then estimated by launching particles from a variety of positions and angles from the outer wall. Results of this model are shown in Fig. 2.4-2 for Shot 73182. During the VH-phase, the penetration fraction $\left(R_{p}\right)$ decreases dramatically due to the increasingly robust SOL shown by the $n_{e}$ and $T_{e}$ contours. After termination of the VH-mode, the penetration fraction rises sharply as the SOL virtually disappears. This analysis indicates the importance of impurity shielding in maintaining a clean plasma with low radiation.

Boronization during the 1992 campaign has continued to reduce both particle fueling from the wall and impurity influxes. A pulsed glow discharge in conjunction with boronization has become routine and has allowed the application of a more uniform film. Before pulsed glow, the film thickness varied toroidally with an estimated $1 \mathrm{~m}$ e-folding length. With the pulsed glow, an e-folding length of $2.1 \mathrm{~m}$ has been measured from coupons exposed during four boronizations. This data is in agreement with a characteristic length of $2 \mathrm{~m}$ calculated from the RGA diborane partial pressure measurements. A more uniform boron film means that a larger fraction of the graphite 


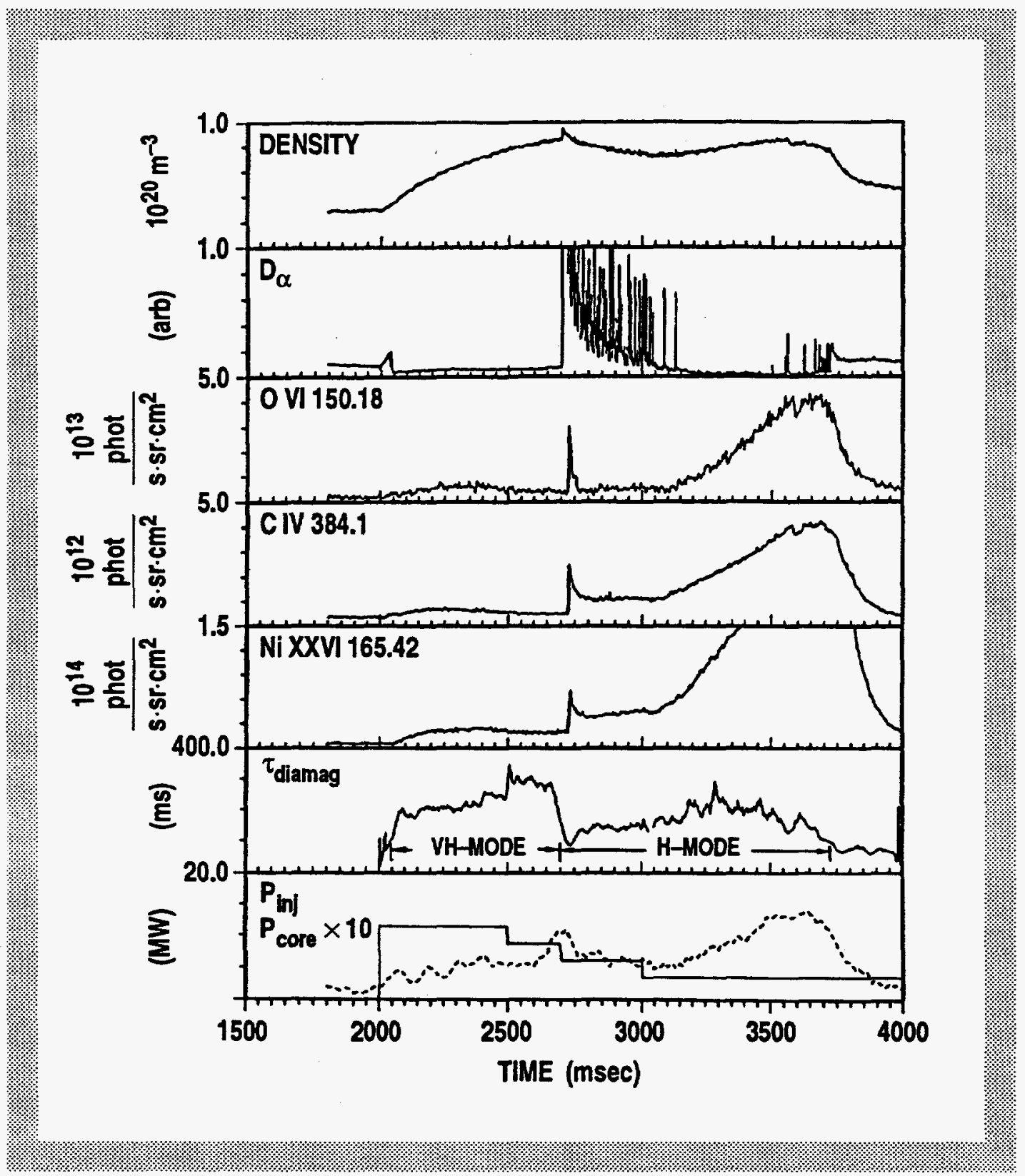

Fig. 24-1. Parameters during a shot which attains VH-mode; interferometric measured $\mathrm{n}_{\mathbf{e}}, \mathrm{D}_{\alpha}$, spectroscopic signals, $\tau_{E}$, and the injected and radiated power.

and Inconel walls have been covered using the two diborane injectors. This, in turn, reduces impurity generation and, in particular, physical and chemical sputtering of carbon.

VH-mode performance has improved in 1992, at least in part due to the improved boron film application discussed above. The power threshold to obtain VH-mode has been reduced as 


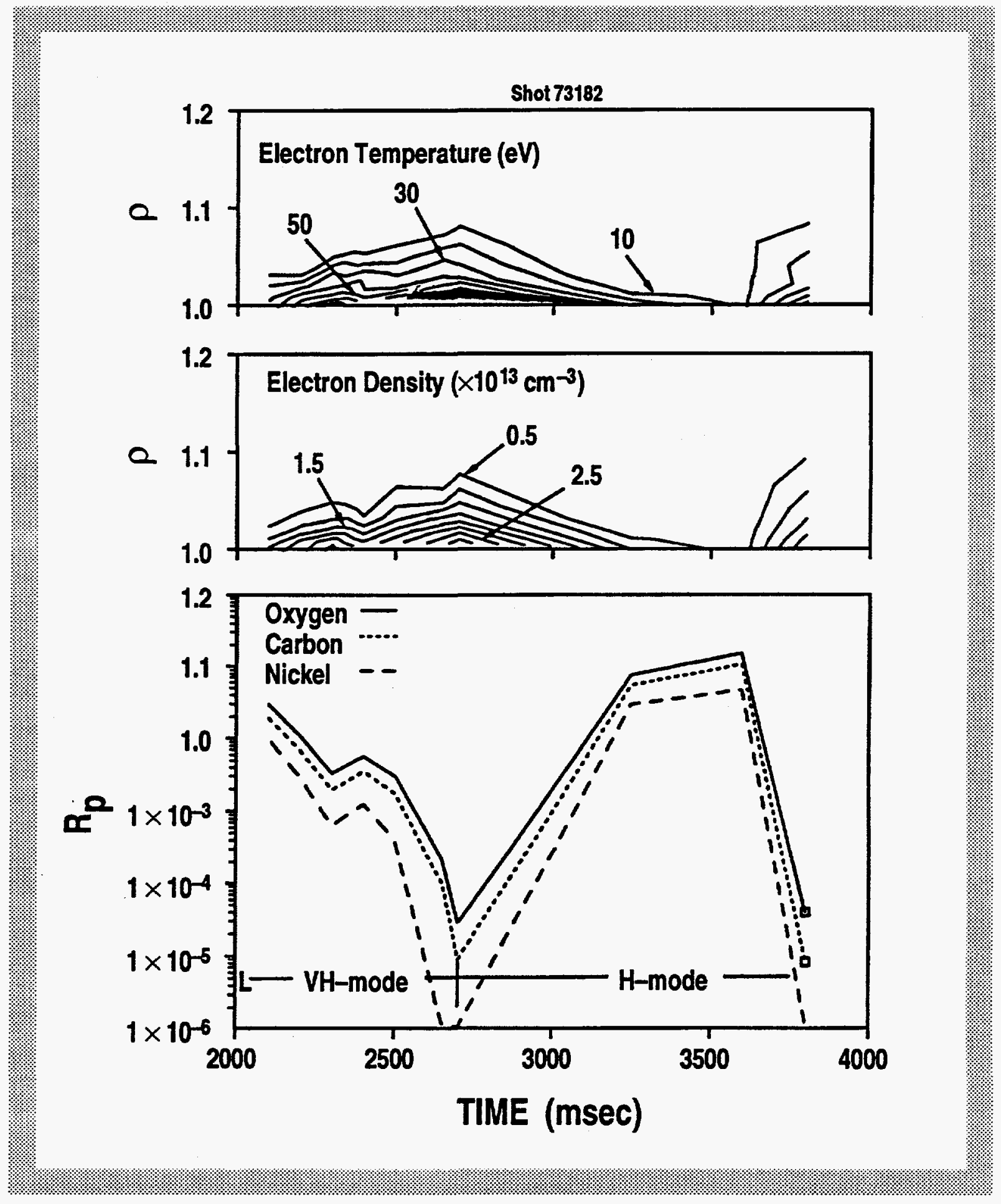

Fig. 2.4-2. Contour plot of scrape-off $T_{e}$ and $n_{e}$ as a function of effective radial localities $r / a=p$ and time, along with the results of the 2D penetration model for carbon, oxygen and nickel, showing the fraction of launched neutral C, 0 , Ni particles that make it past the separatrix. 
shown in Fig. 2.4-3. Plotted in this figure are 1.6 MA VH-mode discharges from the 1991 and 1992 campaigns. The minimum power required to obtain 1.6 MA VH-mode discharges was reduced by more than a factor of two for the 1992 discharges, from 10.5 to $4.3 \mathrm{MW}$.

The wall particle fueling during VH-mode has also been reduced during the 1992 campaign when compared to previous discharges. Particle fueling is calculated from the density rise during the ELM-free portion of $\mathrm{VH}$-mode discharges and is shown in Fig. 2.4-4. The contribution of particle fueling by the neutral beams is low, typically less than $10 \%$ of the total particle fueling. The recycling term, $\mathrm{R}$, is assumed to be 0.5 in this figure. Recycling has not been measured directly, but different assumed values of recycling produce the same qualitative behavior shown in this figure, i.e., there is a general trend of higher energy confinement as the total particle fueling is reduced.

Fig. 2.4-3. The energy confinement time, normalized to JET/OIll-D scaling versus input power. The VH-mode was attained at lower input power following boronization in 1992 (bzen refers to boronization).

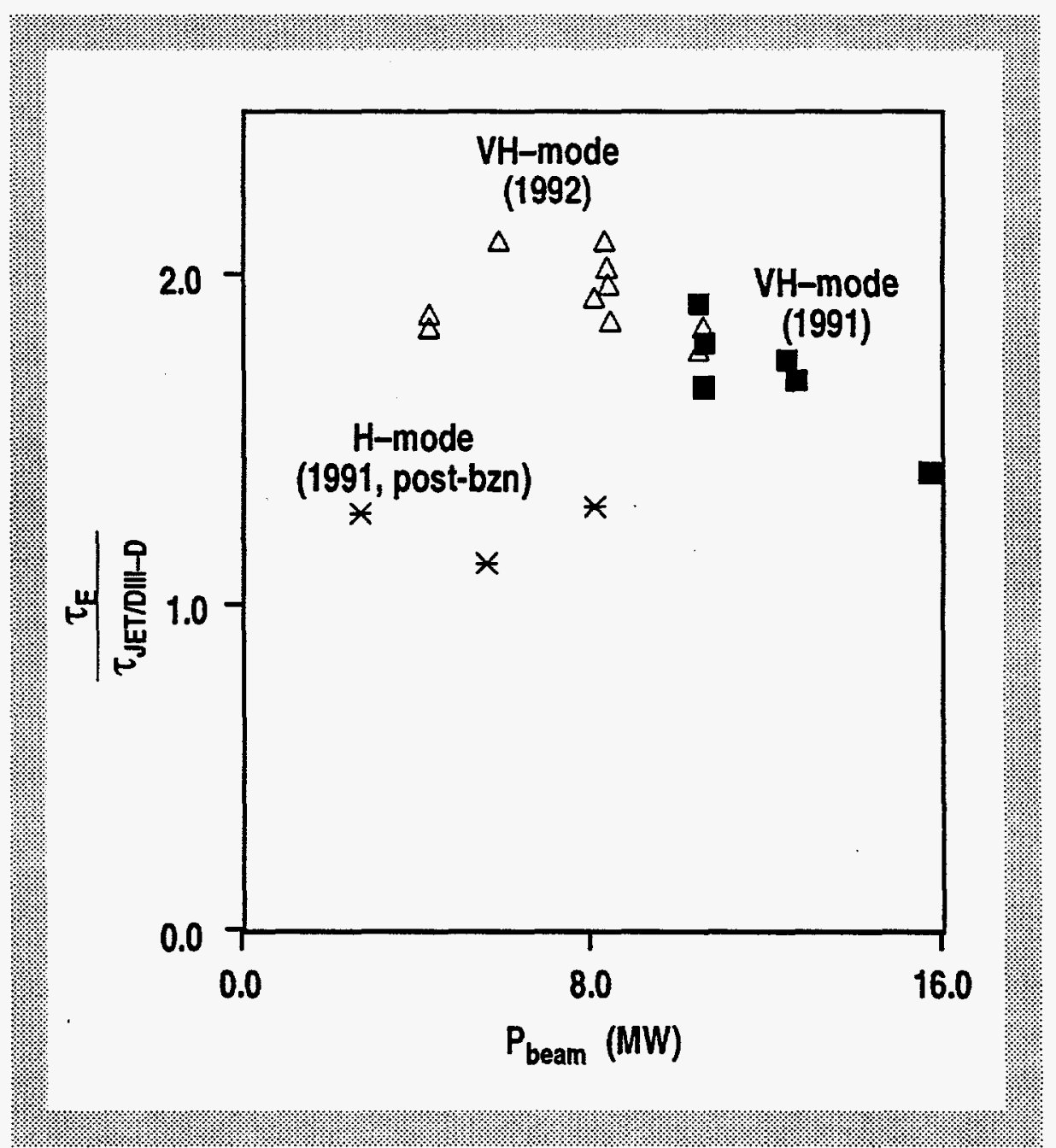


Although impurities have remained low and particle fueling has been reduced in 1992 VH-mode discharges, further confinement enhancements may be possible with boronization improvements and a concomitant reduction in particle fueling and impurity influxes. A new boronization system has been installed to produce a nearly uniform boron film. This system consists of 16 gas injection points on the outer wall. The maximum distance from a gas injector to any plasma-facing surface is approximately $2 \mathrm{~m}$, which is the characteristic length of the boron film using pulsed glow, as discussed previously. This system will be operational for the first boronization in 1993.

Fig. 2.4-4. Energy confinement increases with lower particle fueling influx (czn refers to carbonization).

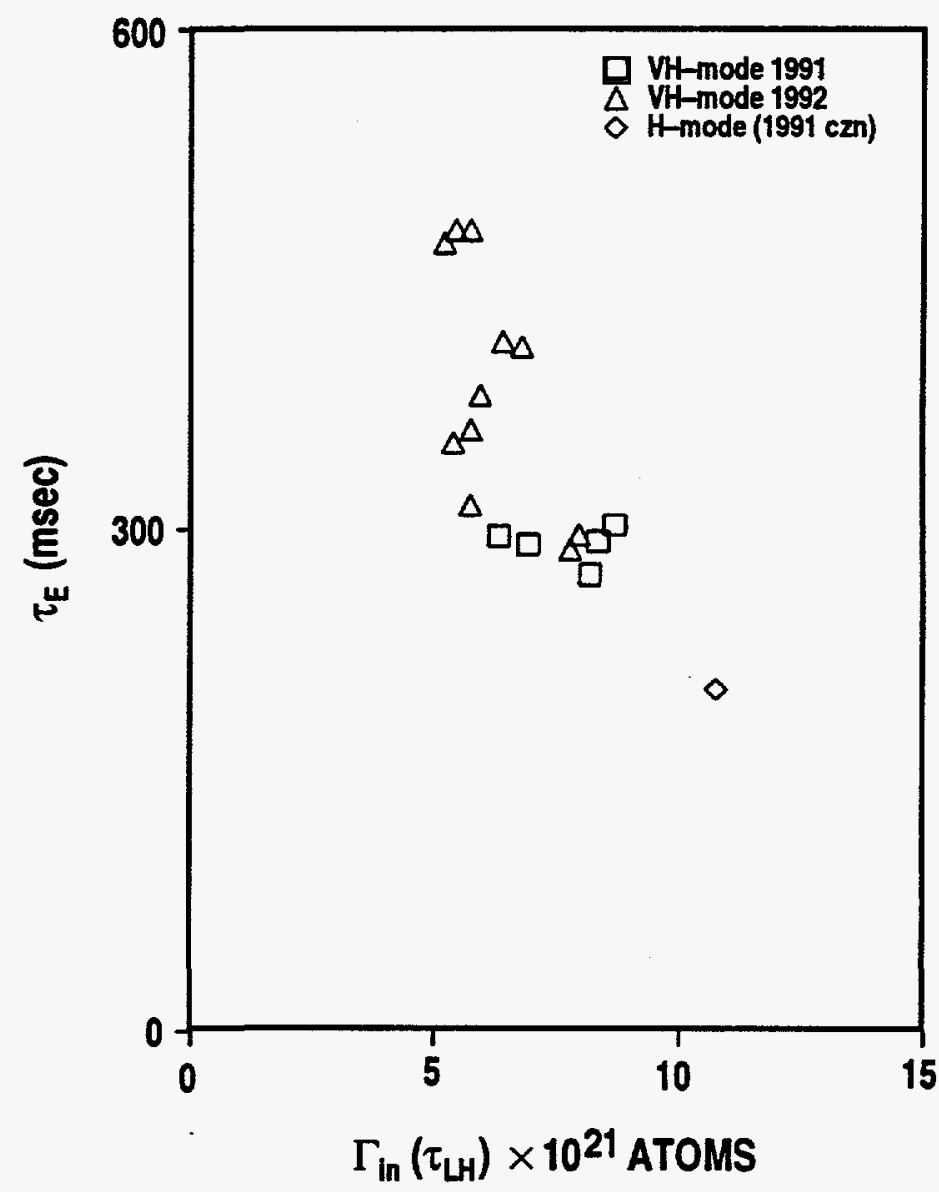



SECTION 3

\section{ADVANCED TOKAMAK STUDIES}





\section{ADVANCED TOKAMAK STUDIES}

\subsection{OVERVIEW}

The objective of the planned research in advanced tokamak development is to establish improved tokamak operation through significant improvements in both the stability factor and confinement quality and bootstrap current fraction. The tools identified for these improvements are localized radio frequency (rf) current profile control, if and neutral beam heating for pressure profile control, and control of plasma rotation and of the plasma boundary conditions. We report here research in $\mathrm{H}$-mode confinement, modifications of the current profile to achieve higher confinement, a new regime of improved confinement (VH-mode), and rf noninductive current drive.

Some key achievements in the study of advanced tokamaks are:

- Time history of the L-to-H transition supporting the theory that gradients in the radial electric field, $\mathrm{E}_{\mathrm{r}}$, lead to decreased turbulence and improved confinement.

- Observations of a correlation between $\mathrm{E}_{\mathrm{r}}$ and fluctuations in the core plasma.

- Measurement of main ion toroidal and poloidal rotation during $\mathrm{H}$-mode.

- Improved understanding of $\mathrm{VH}$-mode.

- Improved confinement by current and elongation ramps.

- Detailed analysis of fast wave current drive (FWCD) experiments with up to $160 \mathrm{kA}$ of noninductive current in a $400 \mathrm{kA}$ discharge, including the identification of differences in the sawteeth behavior, $\ell_{i}$, and the surface loop voltage between the co- and counter-FWCD phasings. 


\subsection{H-MODE CONFINEMENT}

The latest results from DIII-D clearly show that the region just inside the separatrix where the electric field shear develops is also the region where the density fluctuations decrease and where the transport improves. This behavior is illustrated in Fig. 3.2-1. In Fig. 3.2-1(a), $E_{r}$ and the poloidal rotation $v_{\theta}$ for $C$ VII change right at the transition in a localized region just inside the separatrix. The $E_{r}$ value is inferred from the radial force balance equation for C VII. Also shown in Fig. 3.2-1(a) is a curve showing the change in the density fluctuations as revealed by the reflectometer system. The locations of the reflectometer points are determined by using electron density profiles from the multipulse Thomson scattering system. Although interpretation of reflectometer signals can have significant complications, the plot in Fig. 3.2-1(a) indicates a significant change in the density fluctuations in the same narrow region inside the separatrix where the electric field shear develops. Far-infrared (FIR) scattering results have confirmed that the density fluctuations just inside the separatrix decrease right at the L-to-H transition.

As is illustrated in Figs. 3.2-1(b) and 3.2-1(c), both the electron and ion particle and energy confinement improve in the region where the electric field shear is created and where the fluctuations decrease. Density and temperature profiles all steepen in this region after the transition, indicating a confinement improvement. In addition, initial measurements with the fast, moveable Langmuir probe system have shown a definite decrease in the fluctuation-driven particle flux at the separatrix in $\mathrm{H}$-mode relative to L-mode.

Not only is there a spatial correlation between increased shear in $E_{r}$, decreased density fluctuations and improved transport, there is also a temporal correlation. As is shown in Fig. 3.2-2, on DIII-D, at the edge, the poloidal and toroidal rotation speeds of C VII, the inferred $E_{\mathrm{r}}$ and the reflectometer fluctuation power all show dramatic changes at the time that the drop in the divertor $D_{\alpha}$ signal begins. Reflectometer data show that the fluctuation decrease and the beginning of the $D_{\alpha}$ drop are coincident within $0.1 \mathrm{msec}$. Within the $0.5 \mathrm{msec}$ measurement accuracy of the spectroscopic system, the initial step in $\mathrm{E}_{\mathrm{r}}$ also occurs at the same time.

The time sequence of events at the L-to-H transition demonstrates that the change in $E_{r}$ is not a consequence of the change in edge profiles caused by the confinement improvement. As is illustrated in Fig. 3.2-3, a substantial change in the electric field takes 

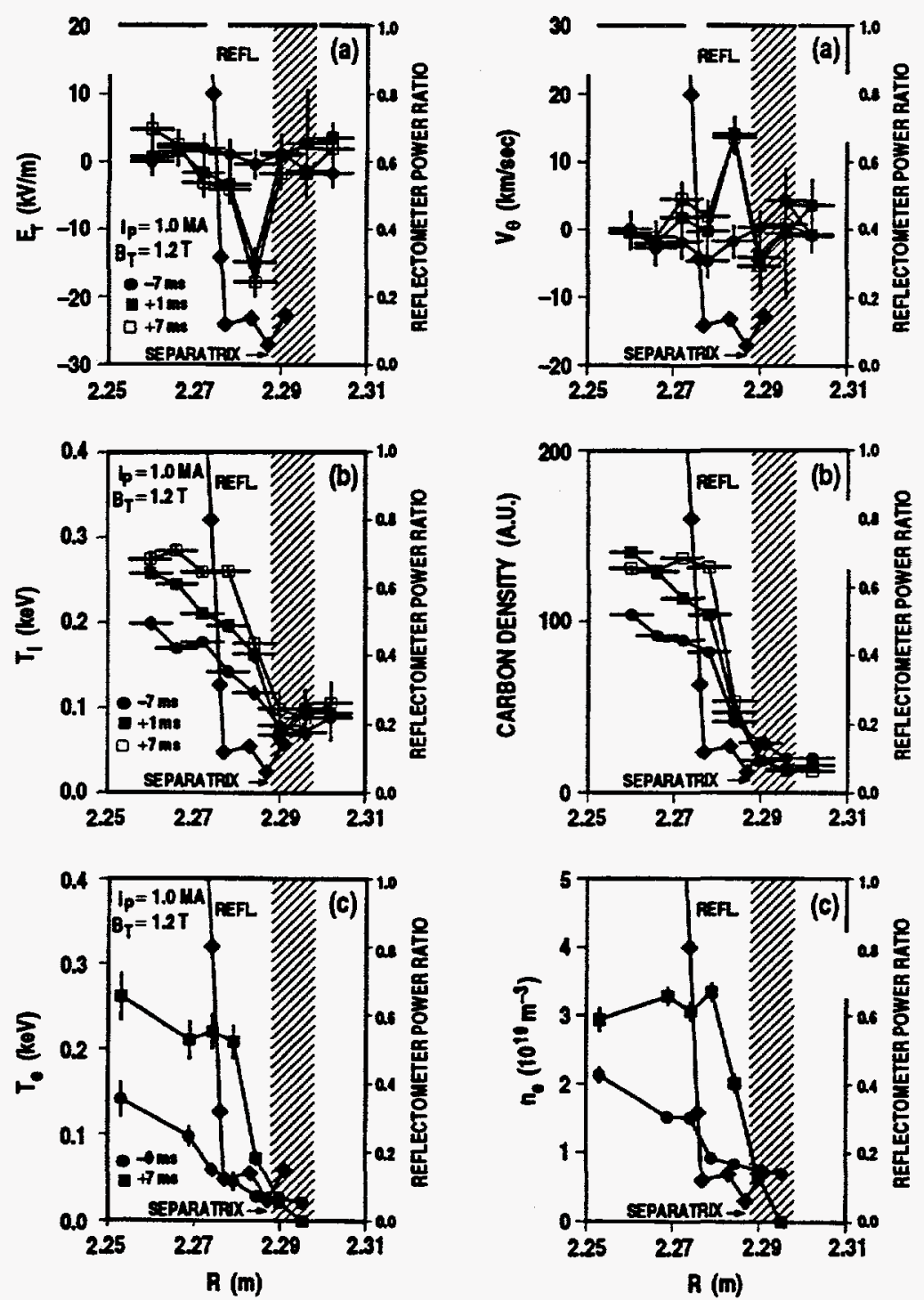

Fig. 3.2-1. Plots of various plasma profiles in DHI-D near the plasma edge at times relative to the L-to-H transition. The shaded region gives the best estimate of the separatrix location as determined from MHD equilibrium analysis. In addition to the profiles labeled on the left-hand vertical axis, each box contains a plot showing the change in the fluctuation power detected by the 0 -mode and $X$-mode reflectometers (righthand vertical scale). This is the ratio of the reflectometer power just after the transition to that measured just before the transition. The reflectometer power is the amplitude of the signal integrated from $75 \mathrm{kHz}$ to the maximum detected frequency ( 400 to $800 \mathrm{kHz}$ ). The frequency integration is based on Fourier analysis over consecutive 0.1 msec intervals. (a) Profiles of poloidal rotation speed of C VII and the radial electric field $E_{\mathrm{r}}$ inferred from the $\mathrm{C}$ VII radial force balance equation as measured by charge exchange recombination spectroscopy. Integration time for the spectroscopic signal was 3 msec. (b) Profiles of the ion temperature and C VII density measured using the same technique. (c) Profiles of electron density and electron temperature measured by Thomson scattering. 


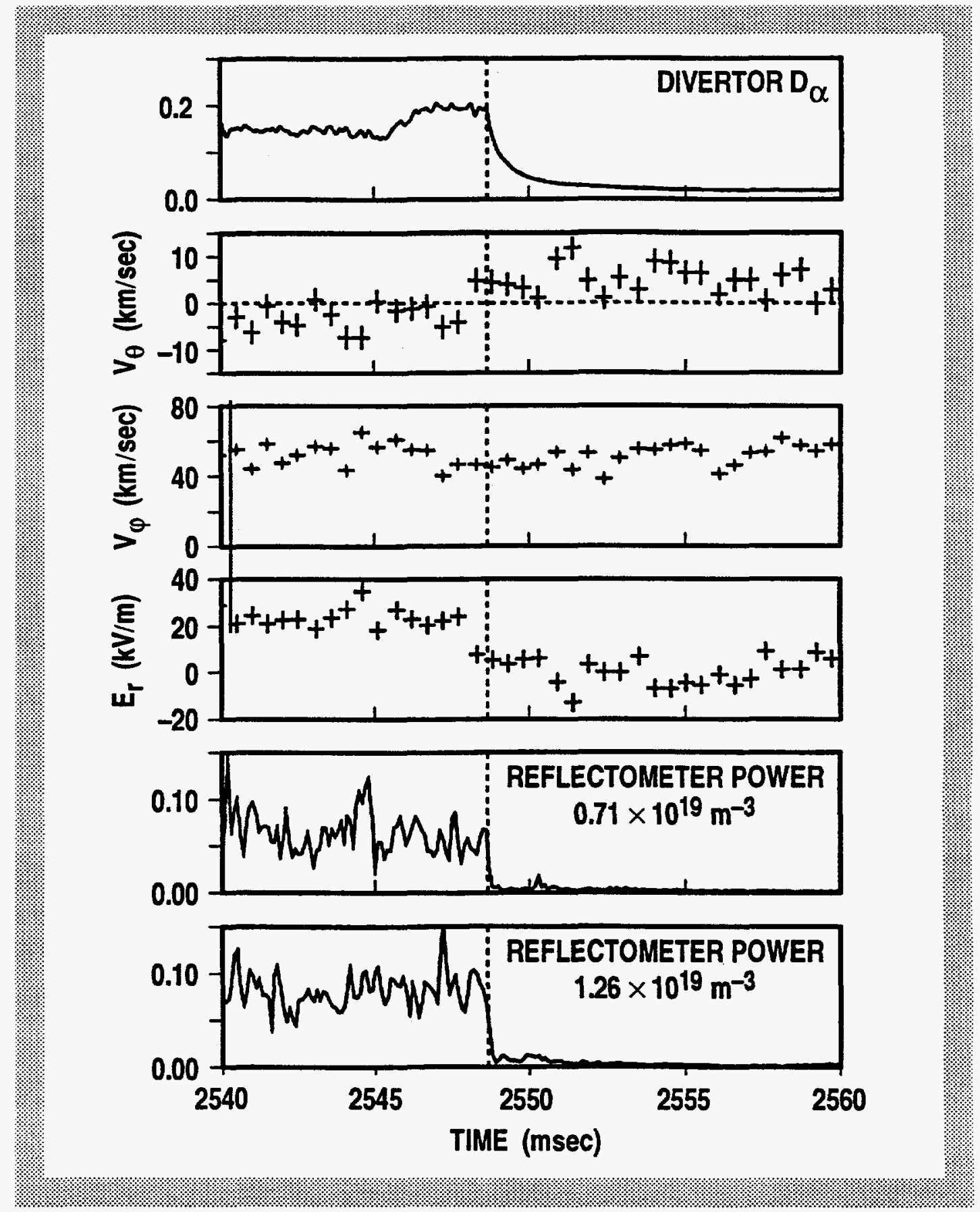

Fig. 3.2-2. Time history across the L-to-H transition in DIII-D of the divertor $\mathrm{D}_{\alpha}$ signal; edge poloidal rotation, toroidal rotation and inferred $E_{r}$ from charge exchange spectroscopy; and reflectometer power. The $D_{\alpha}$ signal and the reflectometer power have effective time resolution of $0.1 \mathrm{msec}$; the spectroscopic signals have a $0.5 \mathrm{msec}$ integration time. The points of the spectroscopic results are plotted at a time which corresponds to the midpoint of the integration time interval. The spectroscopic signals come from the spatial location which corresponds to the minimum of $E_{r}$. 


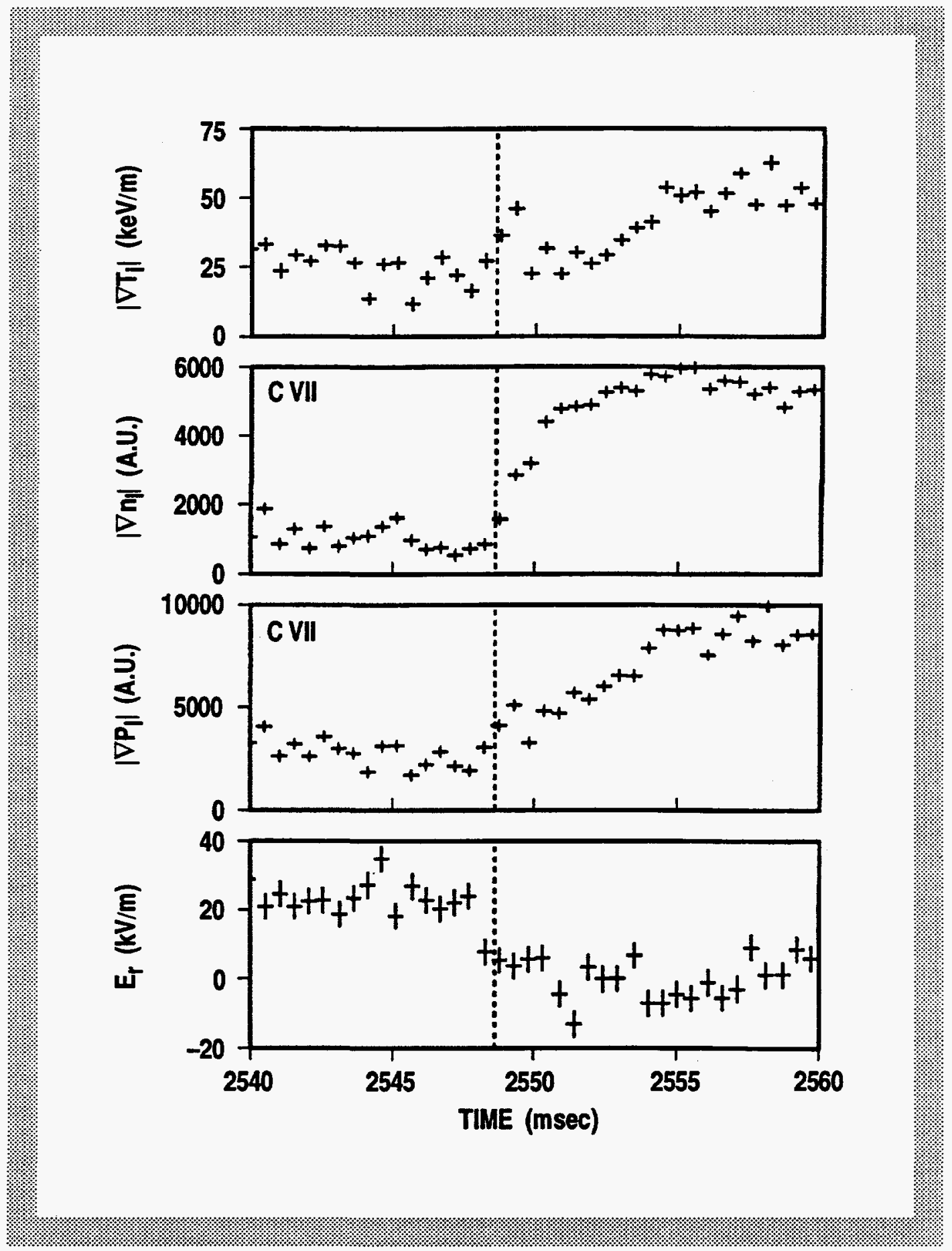

Fig. 3.2-3. Time history across the L-to-H transition in DIII-D of edge ion temperature gradient, C VII density gradient, $C$ VIl pressure gradient and most negative edge $E_{r}$. The spatial location for all the curves is at the point just inside the separatrix where the most negative $E_{\text {, }}$ is seen and where the transport barrier forms. The negative jump in $\mathrm{E}_{\mathrm{r}}$ occurs prior to any significant change in the edge ion gradients. 
place prior to the time when the first change occurs in the local gradients of ion temperature, carbon density, and carbon pressure. In other words, the edge profiles have barely begun to change at a time when a significant $E_{r}$ is already established. This sequence is what one would expect from a model in which the change in $E_{r}$ stabilizes fluctuations, which then leads to a change in the particle and heat fluxes. The usual conservation equations for particle and heat demonstrate that a change in the cross-field fluxes must accumulate for a certain time before the radial profiles exhibit an appreciable response. A model in which the actual changes in the density and temperature profiles produce the change in $E_{r}$ is not consistent with the measured time history. Although the initial, substantial change in $E_{\mathrm{r}}$ seen in DIII-D precedes appreciable changes in the density and temperature profiles, both $\mathrm{E}_{\mathrm{T}}$ and those profiles can continue to evolve for many tens of milliseconds after the transition.

Although it has long been suggested that microturbulence is responsible for anomalous transport, surprisingly little is known about turbulence drive and suppression mechanisms which determine the observed levels of turbulent fluctuations. In DIII-D H-mode discharges, microturbulence has been observed to change in two stages. FIR collective Thomson scattering has confirmed a rapid $(\approx 100 \mu \mathrm{s})$ suppression in edge turbulence at the transition, as well as locally increased edge electric field shear, supporting theoretical models of shear suppression of edge turbulence. During the edge localized mode (ELM) free $\mathrm{H}$-mode, a slower $(20-50 \mathrm{msec})$ reduction of interior turbulence is observed, simultaneous with increased electric field shear and reduced $\nabla \mathrm{n}_{\mathrm{e}}$. In order to separate the roles of electric field and pressure gradients on interior turbulence levels, experiments have been performed whereby the internal electric field is modified while maintaining similar pressure profiles. First, the long-term turbulence reduction observed in $\mathrm{H}$-mode plasmas has been found to be much larger in boronized discharges when strong electric field shear is observed to penetrate deeper into the confinement region than in unboronized discharges. Second, comparison of co- and counter-injection discharges has revealed fluctuation levels increase in co- and decrease in counter-injection L-mode. The reduction is significant at large wave numbers $\left(\mathrm{k}_{\theta} \approx 2 \mathrm{~cm}^{-1}\right)$ and occurs as the internal electric field is driven more negative, while the pressure profile is almost constant. Third, preliminary results have been obtained during experiments where the interior electric field was reduced through the use of "magnetic braking" of the toroidal rotation. As expected, the $\mathrm{E} \times \mathrm{B}$ Doppler shift of the scattered fluctuation spectrum decreased as the electric field was reduced. Additionally, moderate increases in turbulence levels were observed as the internal electric field and 
associated shear was reduced. However, density profiles were strongly modified by ELMs on short time scales, so it is hoped that further analysis will separate these effects.

A recently installed inboard midplane (e.g., high field side) reflectometer system has provided preliminary data indicating a poloidal asymmetric behavior of the density fluctuation suppression at the $\mathrm{L}-\mathrm{H}$ transition. This may have significant implications on both the nature of the transition and the fluctuations as well as the ability to tailor the local transport within a tokamak plasma. Further characteristics of this topic will be actively pursued in more extensive experiments.

Neoclassical theory of ion rotation implies that in the edge region of $\mathrm{H}$-mode plasma, a significant difference in poloidal rotation between the main ion and the impurity ion exists while many theories of $\mathrm{L}-\mathrm{H}$ transition do not distinguish the main ion rotation from the impurity ion rotation. Although it is highly desirable to test the predictions of the neoclassical theory, main ion rotation is usually difficult to measure. By employing helium plasma with modulated deuterium beam injection, however, we were able to probe rotational motion of both the main ion and the impurity ion under the same plasma conditions by means of charge exchange recombination (CER) spectroscopy.

The rotation measurements produced several significant results. Substantial $(\sim 30 \mathrm{~km} / \mathrm{sec})$ main ion poloidal rotation in the direction of ion-diamagnetic drift is observed in the edge region of $\mathrm{H}$-mode helium plasma, while impurity ion poloidal rotation is rather small and in the usual direction of electron-diamagnetic drift, opposite to the main ion poloidal rotation. Toroidal rotation of the main ion remains large $(-100 \mathrm{~km} / \mathrm{sec})$ even to the last closed flux surface, a factor of 2-3 greater than the impurity ion rotation right at the edge of the plasma. Further into the plasma they agree. The deduced radial electric field, consisting of the $\mathrm{V} \times \mathrm{B}$ term and the pressure gradient term, is roughly the same for the main and the impurity ions, exhibiting the usual $\mathrm{H}$-mode negative well in the edge region. It is shown that the neoclassical theory qualitatively predicts the signs and the difference of the main ion and the impurity ion poloidal rotation, but quantitatively does not predict the large main ion poloidal rotation. Temperature profiles of the main ion species $\left(\mathrm{He}^{++}\right)$and the impurity ion species $\left(\mathrm{C}^{+}, \mathrm{B5}^{+}\right)$were shown by measurement for the first time to be quite similar throughout the plasma cross section, as theory predicts they should. 


\subsection{IMPROVED CONFINEMENT THROUGH MODIFICATION OF THE CURRENT PROFILE}

Improverrent in the energy confinement time has been obtained by modifying the current density profile from the relatively broad shape obtained in standard tokamak discharges to a more peaked shape. The peaked current density profile is produced with either a rapid negative ramp in the total plasma current or a rapid increase in the discharge elongation. Using these techniques, up to a factor of 1.8 improvement in the normalized thermal energy confinement time, $\tau_{\mathrm{th}} / \mathrm{I}_{\mathrm{p}}$, has been obtained in both L-mode and H-mode discharges. The highest confinement produced through peaking of the current profile is in $\mathrm{H}$-mode discharges produced with the elongation ramp technique. In these discharges, the thermal energy confinement time is up to 1.8 times the value predicted for $\mathrm{H}$-mode by the Joint European Tokamak (JET)/DIII-D H-mode energy confinement scaling relation and $\tau_{\mathrm{E}}$ is up to three times the value predicted for L-mode by the ITER-89P scaling relation.

In the past year, it has been shown that the observed improvements in confinement are correlated with increases in local values of the average poloidal magnetic field, $\left\langle B_{p}\right\rangle=\int B_{p} d l / \int d l$ where the integral is along a flux surface, and the magnetic shear, $S=\rho(d q / d r) / q$ where $\rho$ is the normalized minor radius, and $q$ is the safety factor. The experimentally determined local single fluid thermal diffusivity, $\chi_{\text {eff }}$, is correlated with the time evolution of the local value of $1 /\left\langle\mathrm{B}_{\mathrm{p}}\right\rangle$. Thermal transport that depends on the local value of $1 /\left\langle B_{p}\right\rangle$ can explain both the commonly observed increase in $\tau_{E}$ with total plasma current and the dependence of $\tau_{E}$ on the shape of the current profile.

Figure 3.3-1 illustrates the time evolution of the local diffusivity in a discharge with a negative current ramp and shows the correlation with the time evolution of the local poloidal magnetic field. The data shown in the figure were obtained in an L-mode, $\kappa=1.7$, inside wall limited discharge with constant neutral beam heating power and a current ramp from 2 to $1 \mathrm{MA}$ during the period 2.5 to $3.2 \mathrm{sec}$. During the current ramp, the internal inductance increases from 1 to approximately 2.2 , and during the $1 \mathrm{sec}$ period following the current ramp, the current profile relaxes and the internal inductance drops to approximately 1.4. During this relatively slow current ramp, and in the following profile relaxation phase, increases in $\chi_{\text {eff }}$ occur across the entire radial profile of the discharge. There is, though, a delay between the increase in $\chi_{\text {eff }}$ in the outer portion of the discharge and the increase closer to the center. This delay is shown in the figure through a 
Fig. 3.3-1. For a discharge with a current ramp, (a) the single fiuid diffusivity and (b) the flux surface averaged poloidal field versus tiime at $\rho=0.5$ (circles) and $\rho=0.8$ (squares). In (a) the dashed line is a best fit to $\chi_{\text {eff }} \propto\langle B p\rangle$.
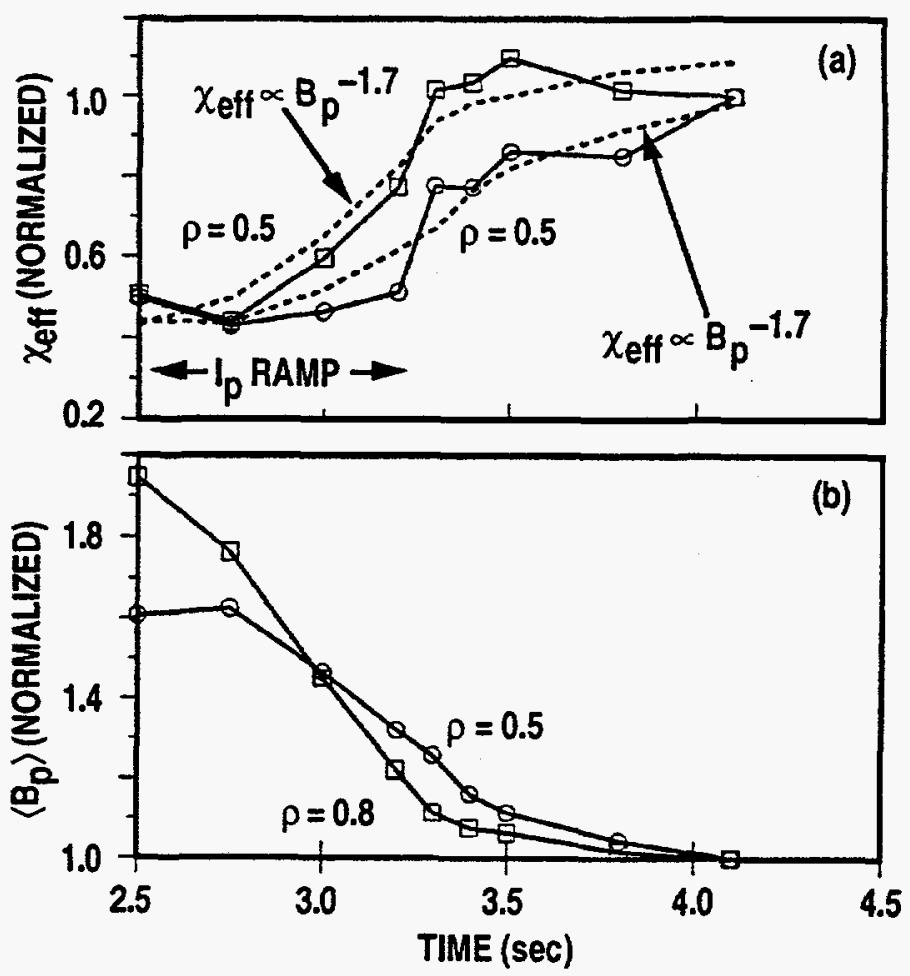

comparison of the time evolution of $\chi_{\text {eff }}$ at $\rho=0.5$ and at $\rho=0.8$. At $\rho=0.8$, most of the change in $\chi_{\text {eff }}$ occurs during the current ramp with little change during the profile relaxation phase, so that $\chi_{\text {eff }}$ has reached approximately the steady-state value by $3.3 \mathrm{sec}$. At $\rho=0.5$, there is little change in $\chi_{\text {eff }}$ during the current ramp and a slow increase during the profile relaxation phase after $3.2 \mathrm{sec}$. At both locations, by the end of the profile relaxation phase, there is a total increase in $\chi_{\text {eff }}$ of approximately a factor of 2 that corresponds to the factor of 2 change in confinement that results from the decrease in the total plasma current.

The value of $\left\langle B_{p}\right\rangle$ has a time evolution similar to that of $\chi_{\text {eff. }}$ At $\rho=0.8,\left\langle B_{p}\right\rangle$ is within $10 \%$ of the steady-state value by $3.3 \mathrm{sec}$ while at $\rho=0.5$, the value of $\left\langle B_{p}\right\rangle$ continues to decrease after that time. The dashed curves in the figure show that $\chi_{\text {eff }} \propto 1 /\left\langle B_{p}\right\rangle \alpha$ provides a good approximation to the value of $\chi_{\text {eff }}$ where the best value of a is between 1.4 and 1.7 depending on the value of $\rho$. 
There is also a change in the radial profile of the radial electric field $\left(E_{\mathrm{T}}\right)$ that is correlated with the changes in the current profile. $E_{r}$ is inferred from the measured profiles of the impurity ion rotation velocity, the local magnetic field strength, and the local impurity ion pressure gradient. Here we consider only the component $E_{r}=v_{T} B_{p}$ where $v_{T}$ is the toroidal rotation velocity. Contributions from the poloidal rotation velocity and pressure gradient terms are believed to be small in the region $0.4<\rho<0.9$. An increase in $\mathrm{v}_{\mathrm{T}}$ is measured during a current ramp and during an elongation ramp, and in both cases $\mathrm{v}_{\mathrm{T}}$ decreases during the profile relaxation phase. Together with local changes in $B_{p}$, this results in a decrease in $E_{r}$ during a negative current ramp and during the current profile relaxation phase of current ramp and elongation ramp discharges. For instance, Fig. 3.3-2 shows that in the current profile relaxation phase of an elongation ramp discharge, as $\ell_{\mathrm{i}}$ decreases, the peak value of $E_{\mathrm{r}}$ decreases from 60 to $35 \mathrm{kV} / \mathrm{m}$ and there is approximately a factor of 4 decrease in the radial derivative, $d E_{\mathrm{r}} / \mathrm{dr}$, in the region $0.4<\rho<0.9$.

The presence of these significant changes in $\mathrm{E}_{\mathrm{T}}$ suggests that sheared flow stabilization of fluctuations is a possible explanation for the observed dependence of confinement on the shape of the current density profile. Stabilization of turbulent fluctuations by spatially varying

Fig. 3.3-2. Radial electric field versus normalized radius for an elongation ramp, $H$-mode discharge at different times during the current profile relaxation phase. The curves are labeled with the values of the intemal inductance at each time.

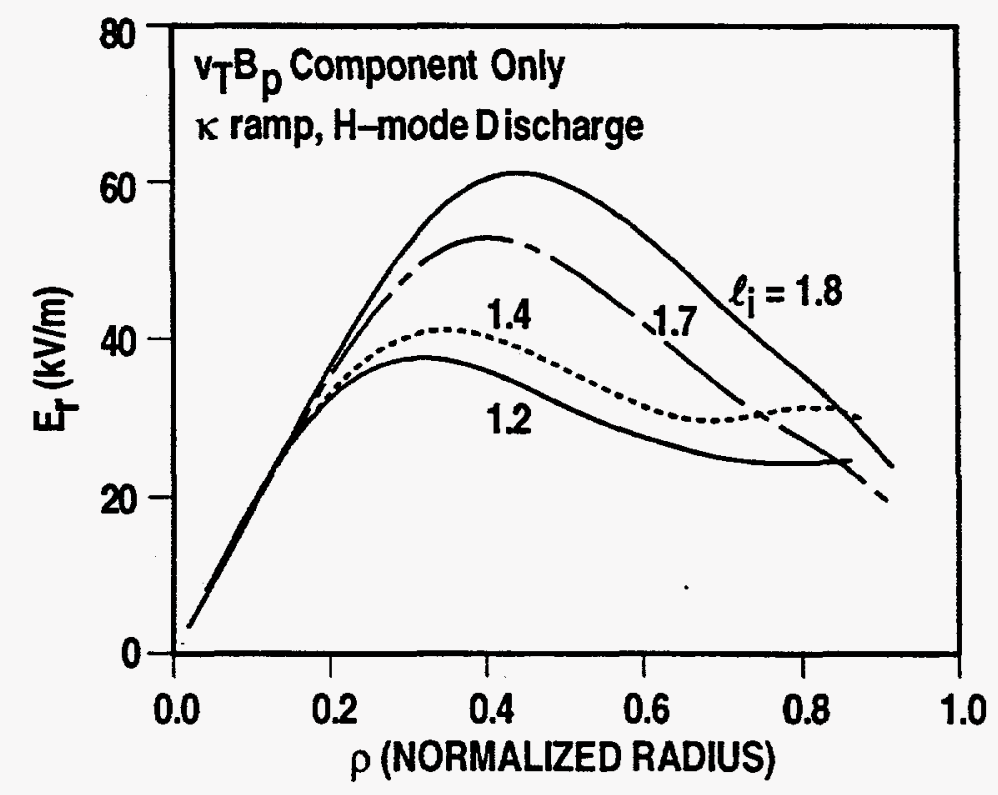


$\mathrm{E}_{\mathrm{r}} \times \mathrm{B}$ flow has been shown to be a good candidate for the cause of the thermal barrier present in $\mathrm{H}$-mode discharges at $\rho>0.9$. It is possible that in the current ramp and elongation ramp experiments, sheared flow stabilization of fluctuations plays a role in the region $0.4<\rho<0.9$. Provided that the magnitude of $d\left(\mathrm{E}_{\mathrm{r}} / \mathrm{B}\right) / \mathrm{dr}$ is larger than the minimum required for stabilization, the variation in $d\left(E_{\Gamma} / B\right) / d r$ observed during changes in the current profile may be sufficient to account for some or all of the observed changes in confinement.

\subsection{VH-MODE}

$\mathrm{VH}$-mode is a regime of improved $\mathrm{H}$-mode confinement which was achieved following boronization of the DIII-D vacuum vessel [3.4-1 through 3.4-3]. Thermal energy confinement times, $\tau_{\mathrm{E}}$, in VH-mode are up to a factor of 2 larger then values predicted by scaling from ELM-free $\mathrm{H}$-mode discharges before boronization [3.4-4], and up to 3.6 times values predicted by ITER-89P L-mode scaling. These high confinement values were obtained at high normalized toroidal beta, $\beta_{\mathrm{N}} \equiv \mathfrak{B}_{\mathrm{T}} /(\mathrm{I} / \mathrm{aB})>3$, and relatively high plasma currents $\mathrm{I} \leq 2 \mathrm{MA}$. This resulted in the highest fusion plasma performance achieved to date on DIII-D, $\mathrm{n}_{\mathrm{D}}(0) \mathrm{T}_{\mathrm{i}}(0) \tau_{\mathrm{E}} \approx 2 \times 10^{20} \mathrm{~m}^{-3} \mathrm{keV}$-sec. Work in 1992 has concentrated on improving the understanding of the $\mathrm{VH}$-mode confinement enhancement and on extending the duration of VH-mode.

To improve our understanding of $\mathrm{VH}$-mode confinement, detailed time histories of profiles and fluctuations were obtained in $\mathrm{H}$ - and $\mathrm{VH}$-mode discharges. The variation of confinement enhancement factor as a function of plasma shape was also explored. Diagnostics integral to these studies were the new eight-laser multipulse Thomson scattering system for $T_{e}$, and $n_{e}$ profiles, charge exchange recombination spectroscopy for $T_{i}, V_{t o r}, V_{p o l}$ and radial electric field profiles, motional Stark effect measurement of field line pitch for determining plasma current density profile, and FIR scattering for density fluctuations.

The operational requirements for $\mathrm{VH}$-mode obtained in previous work [3.4-2] are: (1) boronization, (2) $\mathrm{P}_{\mathrm{NBI}}$ above a threshold level (4 MW in recent discharges), (3) low target density, (4) double-null divertor configuration, (5) $\mathrm{q}_{95} \geq 4$, and (6) moderate plasma current $1.0 \leq I_{p} \leq 2.0 \mathrm{MA}$. Some of these requirements can be understood from the following observations. The energy confinement time increases gradually in VH-mode on a transport time scale. When ELMs begin or a $\beta$ limit is reached, the energy confinement enhancement factor is either clamped or reduced. The rate of increase of the energy 
confinement enhancement factor increases with increasing neutral beam power and also increases as $\mathrm{I}_{\mathrm{P}} / \mathrm{B}_{\mathrm{T}}$ increases (this is known to be true only for double-null divertor discharges). Previous results showed that the duration of the ELM-free period decreased with increasing neutral beam injection (NBI) power and with increasing target density in $\mathrm{H}$-mode. Thus, although it was possible to obtain long ELM-free periods at low power before boronization, the development of the energy confinement enhancement factor may have been too slow to reach significant levels before the first ELM. (There is also some evidence that the rate of increase of the confinement enhancement factor increases with improved boronization quality.) In discharges following boronization, however, the ELM-free period again increases when the neutral beam power is above a threshold level. This effect may be related to the large region near the plasma edge with access to the second stable regime for ideal ballooning modes in these discharges. The effect of discharge shape is shown in Fig. 3.4-1. The ELM-free period in the lowest triangularity single-null discharges (standard single-null shape for DIII-D) was so short that energy confinement enhancement factor was likely limited by the early onset of ELMs. The shortness of the ELM-free period in these discharges may be related to the greater difficulty in obtaining a large, second stable region near the separatrix at low triangularity. With the exception of these lowest triangularity single-null divertor points, all other discharges shown in Fig. 3.4-1 reached saturated levels of energy confinement enhancement factor with no ELMs. Thus, although the absence of ELMs is a necessary condition for obtaining high energy confinement enhancement factors in VH-mode, it is not sufficient. The low current limit, $\mathrm{I}_{\mathrm{P}} \geq 1.0 \mathrm{MA}$, is due to the fact that low current discharges studied to date were at full toroidal field, which resulted in a slow development of the confinement enhancement factor, and to the fact that the ELM-free period decreases as the plasma current decreases (consistent with the change in ballooning mode stability). At low $\mathrm{B}_{\mathrm{T}}$, the global $B$ limit may limit the duration of the ELM-free period. Finally at low q, the energy confinement enhancement factor does not appear to reach as high a level, being only 1.3 at $\mathrm{q}_{95}=3$.

Three hypotheses were considered for the cause of the energy confinement enhancement in VH-mode: (1) the extension of the region of high $\mathrm{E} \times \mathrm{B}$ velocity shear further in from the plasma boundary resulting in a larger region of turbulence suppression, (2) the expansion of the region with access to the second-stable regime for ideal ballooning modes, and (3) the expansion of the region with toroidal drift reversal stabilization of trapped particle modes. The improved confinement in VH-mode is most consistent with the 


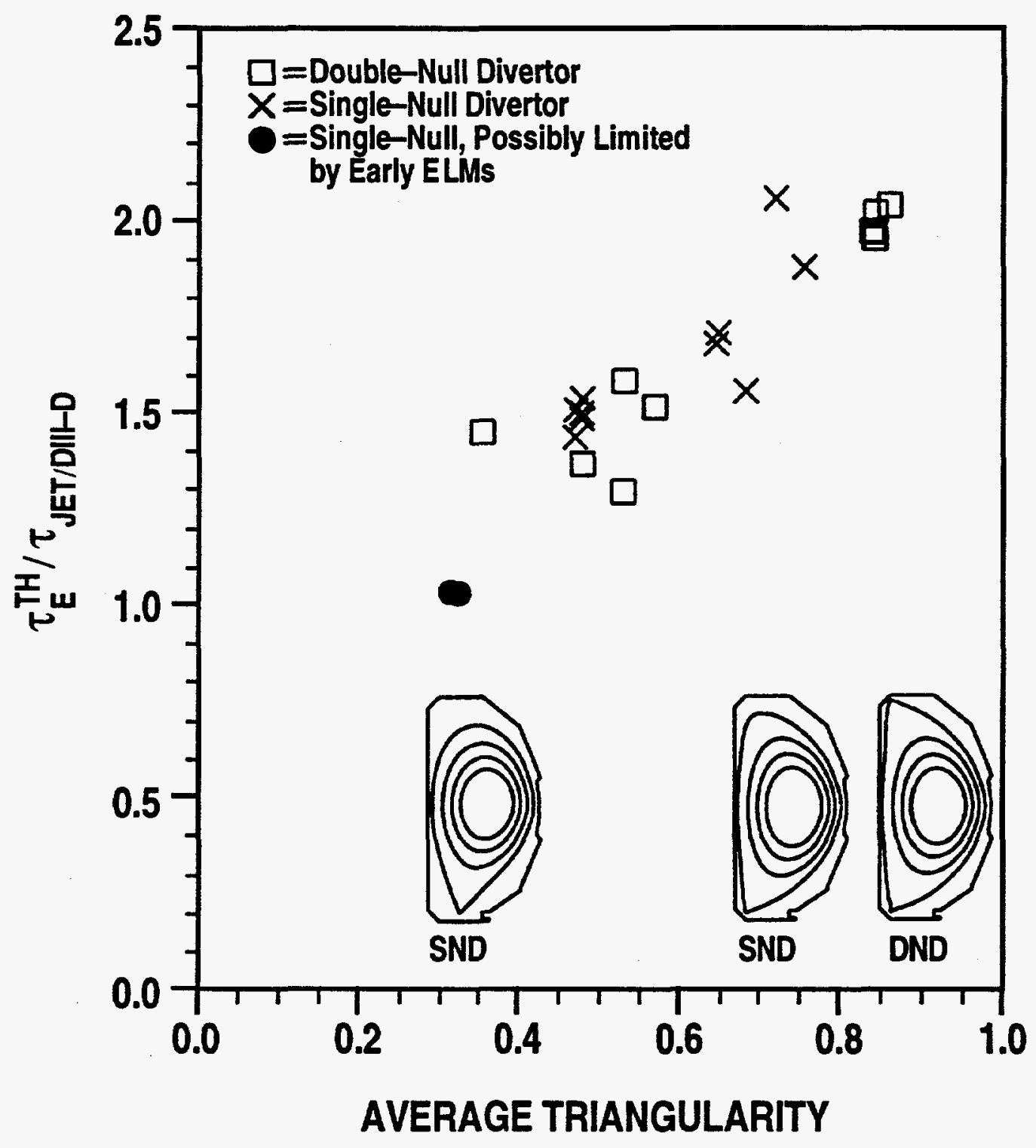

Fig. 3.4-1. Variation of energy confinement time enhancement factor with average triangularity of the separatrix flux surface for single-and double-null discharges. Lowest triangularity single-null enhancement factor may be limited by early onset of ELMs. 
extension of the region of high $\mathrm{E} \times \mathrm{B}$ velocity shear. Figure 3.4-2 shows the penetration of the $\mathrm{E} \times \mathrm{B}$ velocity shear in a $\mathrm{VH}$-mode discharge. The region of increased $\mathrm{E} \times \mathrm{B}$ velocity shear is consistent with the region of reduced effective thermal diffusivity in VH-mode. Also shown in Fig. 3.4-2 is a comparison of the measured $\mathrm{E} \times \mathrm{B}$ velocity shear with the value required for turbulence suppression based on density fluctuation measurements in the DIII-D, L-mode edge region. Figure 3.4-3 shows a comparison of the results of analysis of a 1.6 MA, 2.1 T, double-null divertor $\mathrm{VH}$-mode which reached a confinement enhancement factor of $\mathbf{2 . 0}$ for these three models. In the case of the drift reversal hypothesis, a region of stability near the plasma edge does increase in size during VH-mode; however, the value of the normalized radius, $\rho$, outside of which stability is expected only reaches 0.9 by the end of the VH-mode phase. This is not large enough to cover the region of reduced diffusivity shown in Fig. 3.4-2. Although the calculated region with access to the second regime of ideal ballooning is significant, reaching $\rho=0.78$, there is little change in the volume with second-stable access (from $\rho=0.83$ to $\rho=0.78$ ) while the energy confinement enhancement factor increases dramatically. It should be pointed out that present stability analysis did not include the effects of resistivity and finite $\mathrm{n}$ modes, which might modify the conclusion as to the importance of ballooning stability in VH-mode confinement. As mentioned earlier, edge second stability may be important in obtaining long ELM-free periods at high neutral beam power, which is necessary for VH-mode. The best agreement with the temporal evolution of the energy confinement enhancement factor is with the $\rho$ value outside of which the Biglari, Diamond, Terry criteria for $\mathrm{E} \times \mathrm{B}$ velocity shear turbulence suppression is met. It is also found that discharges in Fig. 3.4-1 with a higher energy confinement enhancement factor have a larger $\mathrm{E} \times \mathrm{B}$ velocity shear penetration.

In VH-mode discharges which exceed a confinement enhancement of 1.5 , there is a rapid jump observed in the confinement enhancement factor as shown in Fig. 3.4-4. To within the accuracy of the $\tau_{\mathrm{E}}$ temporal evolution, this rapid increase in confinement corresponds to the time of increase in the toroidal rotation velocity shear (of impurity ions) which is designated the spin-up time. In the region inside $\rho=0.9$, where the greatest improvement in radial electric field is observed in $\mathrm{VH}$-mode, the toroidal velocity is the primary contributor to $E_{I}\left[E_{T}=v_{I} \times B+\left(1 / n_{I} \rho_{I}\right) \nabla P_{I}\right]$. The change in the toroidal rotation and subsequent penetration of the $\mathrm{E} \times \mathrm{B}$ velocity shear is due to the elimination of a flat spot in the toroidal rotation profile between $\rho=0.6$ and $\rho=0.8$. At the spin-up time, there 


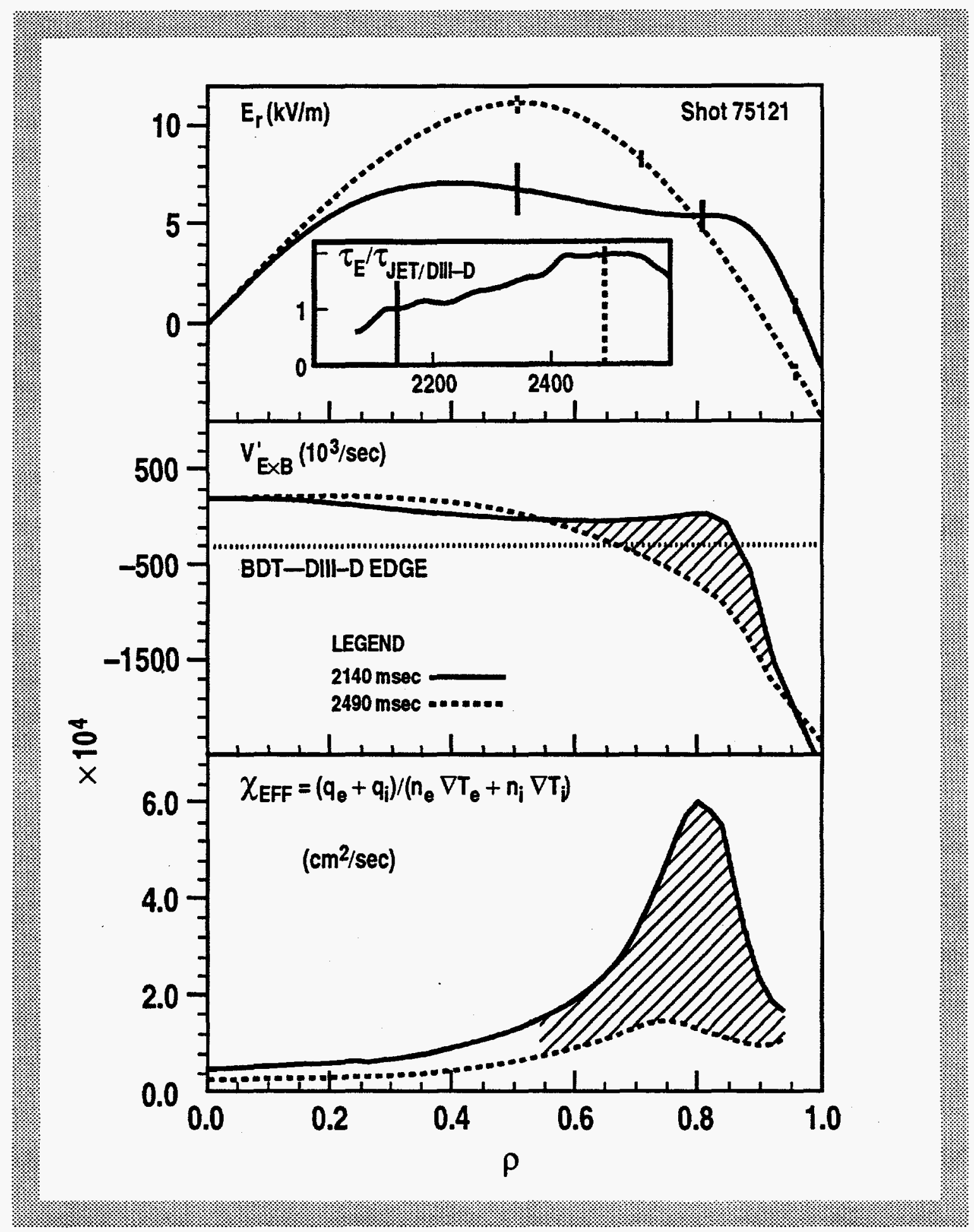

Fig. 3.4-2. Change in the radial electric field, $E_{r} E \times B$ velocity shear, $V^{\prime} E \times B$, and effective thermal diffusivity, $\chi_{\text {eff }}$ during a $\mathrm{VH}$-mode discharge. The $E \times B$ velocity shear increases in the region of large diffusivity reduction. Also shown is the $E \times B$ velocity shear required for turbulence suppression based on turbulence measurements in the DIII-D L-mode edge (dotted line). 


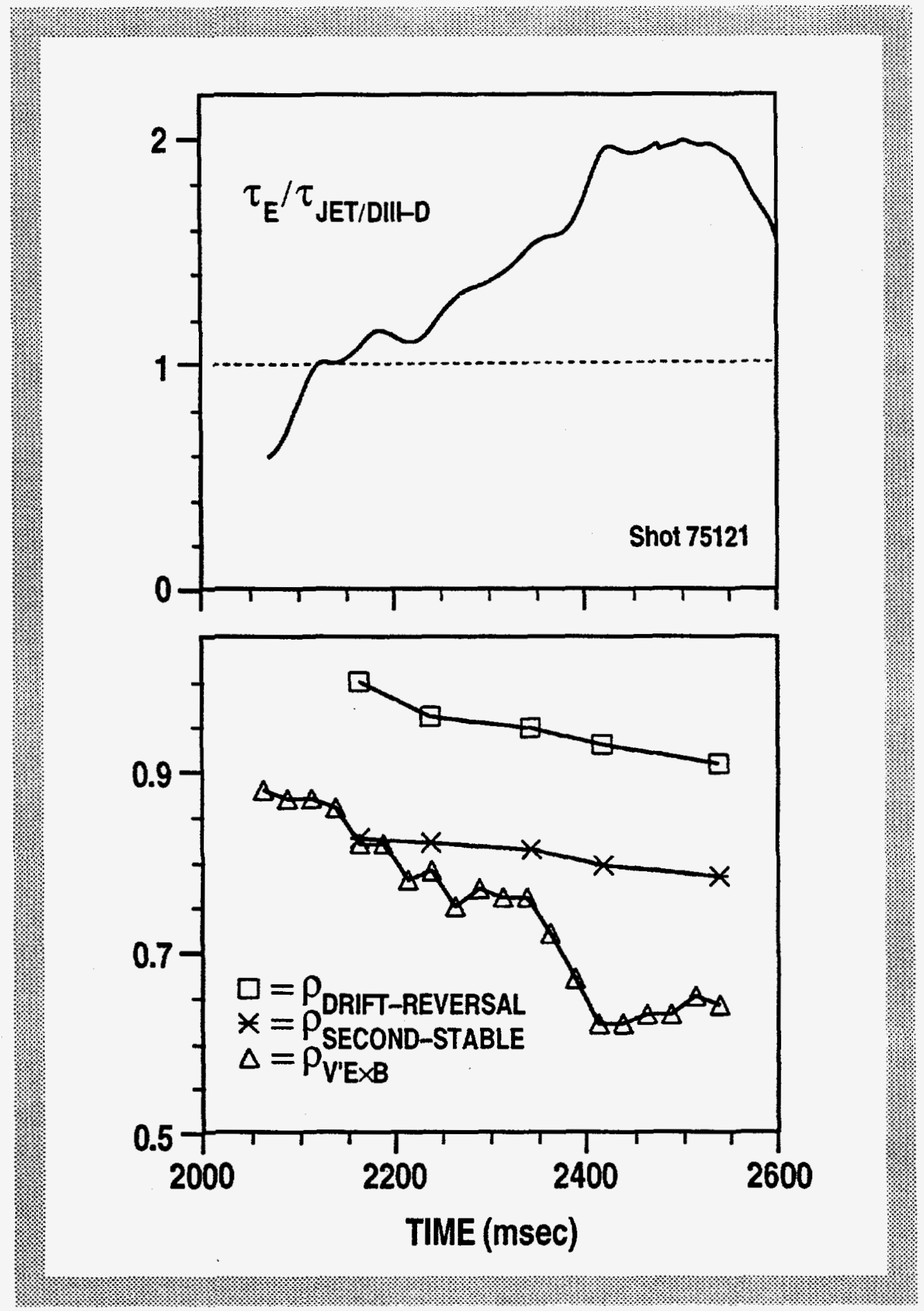

Fig. 3.4-3. Energy confinement time enhancement factor, $\tau_{E} / \tau_{\text {JETIDII-D }}$ and quantities corresponding to the three models for confinement enhancement improvement considered. PDRIFT-REVERSAL is the $\rho$ value outside of which drift reversal stabilization of trapped particle modes is expected, PSECONDSTABLE is the $\rho$ value outside of which the discharge has second stable access, and $P V^{\prime} E \times B$ is the $\rho$ value outside of which the Biglari, Diamond, Terry criteria for $E \times B$ velocity shear turbulence suppression is met. 


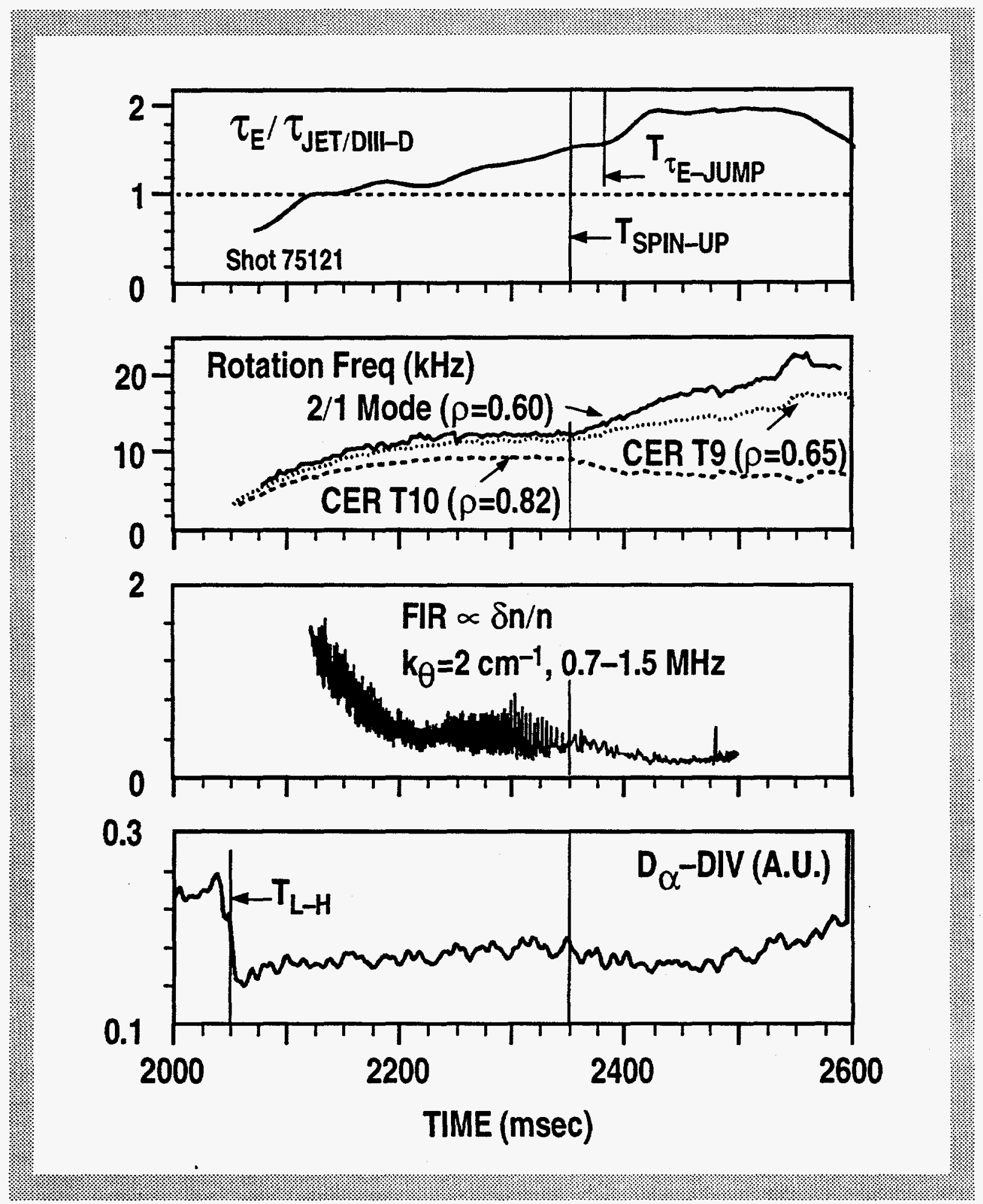

Fig. 3.4-4. An increase in the shear in the toroidal rotation speed occurs near the time of a rapid jump increase in the energy confinement enhancement factor. At this spin-up time, density fluctuations bursts observed on the FIR scattering diagnostic are suppressed, and there is a small drop in the divertor recycling light. 
is an disappearance of bursts observed on the FIR scattering as shown in Fig. 3.4-4. From the measured $\mathrm{E} \times \mathrm{B}$ velocity profile, these bursts are found to originate from the region near the location of the toroidal rotation flat spot. These bursts cause transfer of momentum from $\rho=0.6$ and $\rho=0.8$, and for that reason are designated as momentum transfer events (MTEs). High frequency MTEs may be responsible for the flat spot in the toroidal rotation profile and their elimination may allow the $\mathrm{E} \times \mathrm{B}$ velocity shear to penetrate. For discharges which do not show a spin-up, as for example the medium triangularity single-null discharges of Fig. 3.4-1, which only achieve an enhancement factor of 1.5, the MTEs are continuous and high frequency.

The ELM-free phase of a VH-mode discharge is almost always terminated by a global energy loss event, after which $\tau_{E}$ drops to an $\mathrm{H}$-mode level (given by JET/DIII-D scaling) and ELMs begin. An example of a terminating event is shown in Fig. 3.4-5. The event is initiated by a medium $n$ (in this case $n=5$ ) mode observed on poloidal magnetic field pickup probes at the vessel wall. This mode rotates in the electron diamagnetic drift direction and grows on a time scale of 20 to $50 \mu \mathrm{sec}$. In addition to this rapidly growing medium $n$ mode, low $n$ modes $(n=1$ or 2$)$ are observed growing on a time scale of $10 \mathrm{sec}$ of milliseconds before the termination. These slowly growing modes rotate in the ion drift direction (neutral beam injection direction) and are located on internal low order rational surfaces $(q=1,1.5$, or 2$)$. A coupling of the medium $n$ mode with the internal low $\mathrm{n}$ modes may be responsible for the global nature of the termination. Using experimentally determined equilibria, ideal kink stability calculations using the GATO code show edge localized modes, with $n=2,3,4$, to be unstable just before the termination while these modes are stable shortly after the L-to-H transition. The termination occurs for normalized beta, between 2.5 and 3.5 in agreement with the expected beta limit with high edge current density.

Since the VH-mode termination appears to be a $\beta$ limit phenomenon, an attempt was made to extend the $\mathrm{VH}$-mode period by limiting the $\beta$ value. Using the digital plasma control system, the beam power was feedback controlled to hold the diamagnetic flux constant by changing the chopping frequency and/or number of ion sources. This allowed the extension of the ELM-free period to $1 \mathrm{sec}$ and $\tau_{\mathrm{E}} \geq 2 \tau_{\mathrm{JET} / \mathrm{DIII}-\mathrm{D}}$ for up to $0.6 \mathrm{sec}$. These discharges were still terminated with the global event described above but a a low $\beta_{\mathrm{N}}=2.6$, perhaps due to the lack of control and continual build up of the edge current density. 


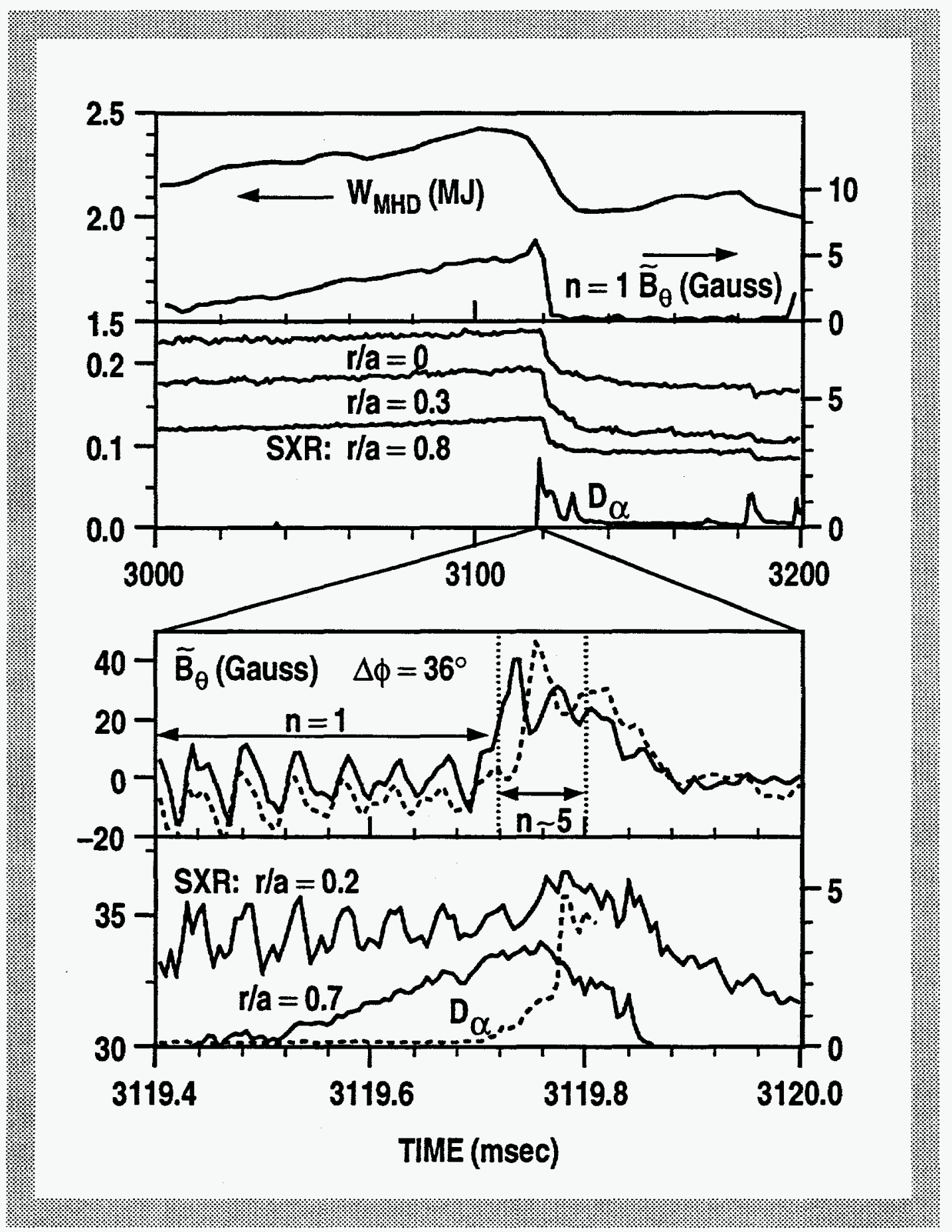

Fig. 3.4-5. Events associated with the termination of ELM-free VH-mode. A rapid drop in plasma energy across the plasma cross section is inferred from the reduction in the soft X-ray, SXR, signals for all radii within a few hundred $\mu \mathrm{sec}$ of the terminating event. An ELM-like spike is seen in the divertor recycling light at the time the SXR signals drop. A slowly growing $n=1$ mode associated with a mode at the at the $q=1$ surface drops rapidly in amplitude at the time of the termination. A rapidly growing $n=5$ mode is observed $100 \mu \mathrm{sec}$ before the termination. 


\section{REFERENCES FOR SECTION 3.4}

[3.4-1] Jackson, G.L., J. Winter, T.S. Taylor, et al., Phys. Rev. Lett. 67 (1991) 3098.

[3.4-2] Jackson, G.L., J. Winter, T.S. Taylor, et al., Phys. Fluids B 7 (1992) 2181.

[3.4-3] Taylor, T.S., T.H. Osborne, K.H. Burrell, et al., in Proc. 14th Int. Conf. on Plasma Physics and Controlled Nuclear Fusion Research, October 1992, Würzburg, Germany, IAEA Report IAEA-CN-56/A-3-1 (1992).

[3.4-4] Schissel, D.P., J.C. DeBoo, K.H. Burrell, et al., Nucl. Fusion 31 (1991) 73. 


\subsection{NONINDUCTIVE CURRENT DRIVE}

\subsubsection{ELECTRON CYCLOTRON HEATING (ECH) FOR FAST WAVE CURRENT DRIVE}

The $60 \mathrm{GHz}$ ECH system was used in the FWCD experiments to heat the electrons to temperatures of $2 \mathrm{keV}$ or greater in order to provide the necessary target conditions for the fast waves (FW). ECH again was launched from the inside of the torus in the $\mathrm{X}$-mode of polarization. Power levels in the range of 0.5 to $1 \mathrm{MW}$ were incident on the plasma. Results from these experiments are described in the next section.

\subsubsection{FAST WAVE HEATING AND CURRENT DRIVE}

FWCD is one of the leading contenders for driving current noninductively near the center of the plasma of the International Thermonuclear Experimental Reactor (ITER) tokamak. Fast waves in the ion cyclotron range of frequencies may be damped directly by the electrons through the processes of electron Landau damping and transit-time magnetic pumping (TTMP). These damping processes are rather weak, but they increase strongly as the electron temperature and beta are raised. This allows the waves to penetrate to and be absorbed near the center, even in a reactor-like plasma of very high electron temperature (unlike the much more strongly damped lower hybrid wave, which is absorbed near the edge under reactor-like conditions). This behavior makes FWs highly suitable for reactor applications, but it makes studies of FW absorption difficult in plasmas with low electron temperature. We take advantage of the ECH system of DIII-D to raise the electron temperature $\left(T_{e}\right)$ and increase the damping strength.

A long-range goal of the DIII-D program is the improvement of the tokamak concept. One possible way to improve the confinement of energy or the beta limit is to optimize the current density profile. Peaking of the current density profile $j(r)$ (as measured by increasing internal inductance $\ell_{\mathrm{i}}$ ) has been shown to accomplish both of the above objectives in nearcircular DIII-D discharges, where the increase in $\ell_{\mathrm{i}}$ is accomplished transiently by ramping the plasma current. This may also be accomplished in steady-state through noninductive current drive techniques like FWCD. Therefore, in order to carry out its long-term mission, an intermediate objective for the DIII-D program is a demonstration of full noninductive current drive with good confinement in a high beta divertor plasma. 
Present experiments represent the first steps of the FWCD program. The experiments are meant to demonstrate the basic physics of the FW interaction with electrons and to advance the numerical models for FW heating and current drive by providing a body of experimental data for comparison. At the low rf powers involved at this stage of the program, large noninductive currents are not expected.

The DIII-D tokamak is well-suited to a study of FWCD. The FW antenna occupies a pair of midplane ports on the outer wall of the vacuum vessel, while the ECH antennas are located on the inboard wall $13 \mathrm{~cm}$ above the midplane of the vessel. The FW antenna has four independently phased current carrying straps spaced $23 \mathrm{~cm}$ between centers. The straps are $11 \mathrm{~cm}$ wide and $45 \mathrm{~cm}$ high. The antenna was made at Oak Ridge National Laboratory (ORNL). The antenna is driven by an rf source with power up to $2 \mathrm{MW}$ and frequency of 30 to $60 \mathrm{MHz}$.

The experiments were performed at $60 \mathrm{MHz}$. With the phasing between straps set to $180^{\circ}$ (" $\pi$-phasing") the power spectrum is symmetric (equal powers are launched in both toroidal directions), and the major peaks are at $n_{\|}= \pm 11$. ( $n_{\|}$is the ratio of the speed of light to the phase velocity of the wave in the toroidal direction so that the smaller $n_{\|}$, the faster the wave.) With $n_{\|} \cong 11$ the wave is resonant with electrons of about $1 \mathrm{keV}$ of energy. For FWCD, the phasing between straps is set to $90^{\circ}$ (" $\pi / 2$ phasing") to generate a toroidally asymmetric spectrum to preferentially drive current in one direction. The forward spectrum peaks at n" $\cong 5.5$ which is resonant with $=4 \mathrm{keV}$ electrons. Accurate numerical models are being developed to understand how the antenna spectrum is transformed into the wave spectrum launched in the plasma.

\subsubsection{FAST WAVE CURRENT DRIVE}

A series of experiments at the end of FY91 and the begining of FY92 with $\pi / 2$ antenna phasing demonstrated for the first time that noninductive FWCD could be obtained. Substantial electron heating and decreases in loop voltage (the total plasma current is held constant by feedback control) were observed during FWCD at toroidal fields of 1.0 and $2.0 \mathrm{~T}$ with or without $\mathrm{ECH}$ preheating. The level of noninductive current drive is measured by comparing the plasma resistance determined from the loop voltage and toroidal current with that calculated from neoclassical resistivity. In the calculation, full measured profiles of $n_{e}, Z_{e f f}, T_{e}$, and $T_{i}$ are utilized. This 
technique was benchmarked by comparing the predicted and observed loop voltages in ohmic and neutral beam-heated discharges. Agreement within $\sim 0.03 \mathrm{~V}$ is typical. During 1992, a detailed analysis of the FWCD data was undertaken. In addition to demonstrating co-current drive in agreement with theoretical models, substantial progress was made in understanding counter-current drive results with FWs.

The largest fractions of noninductively-driven current were observed with $\mathrm{B}_{\mathrm{T}}=1 \mathrm{~T}$, and $\overline{\mathrm{n}}_{\mathrm{e}}=1.0 \times 10^{19} \mathrm{~m}^{-3}$ with ECH preheating. Up to $0.16 \mathrm{MA}$ of noninductively-driven current was observed, with a total $I_{p}=0.40$ MA. A typical discharge of this kind is shown in Fig. 3.5-1(a), along with a comparison of the observed loop voltage with a time-dependent calculation of the loop voltage predicted from the measured profiles and neoclassical resistivity. The calculation includes the bootstrap current, so that the difference between the measured loop voltage and the calculated value indicates the rf-driven current [electron cyclotron current drive (ECCD+FWCD)]. Without ECH preheating, under otherwise similar conditions, a noninductive current (the sum of the FWCD and the bootstrap current) of about $0.135 \mathrm{MA}$ was obtained. Both with and without $\mathrm{ECH}$, the bootstrap current contribution is calculated to be $40 \mathrm{kA}$. The two filled-in squares in Fig. 3.5-1 (a) show the results of a calculation of the loop voltage based only on the observed profiles at those two times. The agreement between the time-dependent and "snapshot" calculations of the surface voltage was found to be usual for co-current FW injection, indicating that the electron temperature and ohmic current profiles evolve slowly enough that the loop voltage at the surface of the plasma. is always equal to the voltage near the magnetic axis. This behavior was found to be quite different in the counter-current drive case, as is shown below. However, for both co- and counter-FWCD, effects resulting from back-emfs have not been taken into account; for coFWCD with ECH preheating, the current drive efficiency may be somewhat underestimated for the relatively short $\mathrm{ECH}$ pulses employed to date.

A discharge with counter-current FW injection is shown in Fig. 3.5-1(b). The ECH antennas are at a fixed angle with respect to the plasma current, so that any ECCD is in the cocurrent direction in both cases. When the FW power is injected after $\mathrm{ECH}$ preheating, the central electron temperature rapidly increases to $4 \mathrm{keV}$. This sudden rise results from partial stabilization of the sawteeth, which was never observed in the co-current drive (CD) cases. Evidence from soft $\mathrm{X}$-ray cameras indicate that the stabilization may result from counter-current drive near the axis momentarily increasing the safety factor above unity near the center of the discharge. The central temperature drops very abruptly at the end of the sawtooth stabilized 


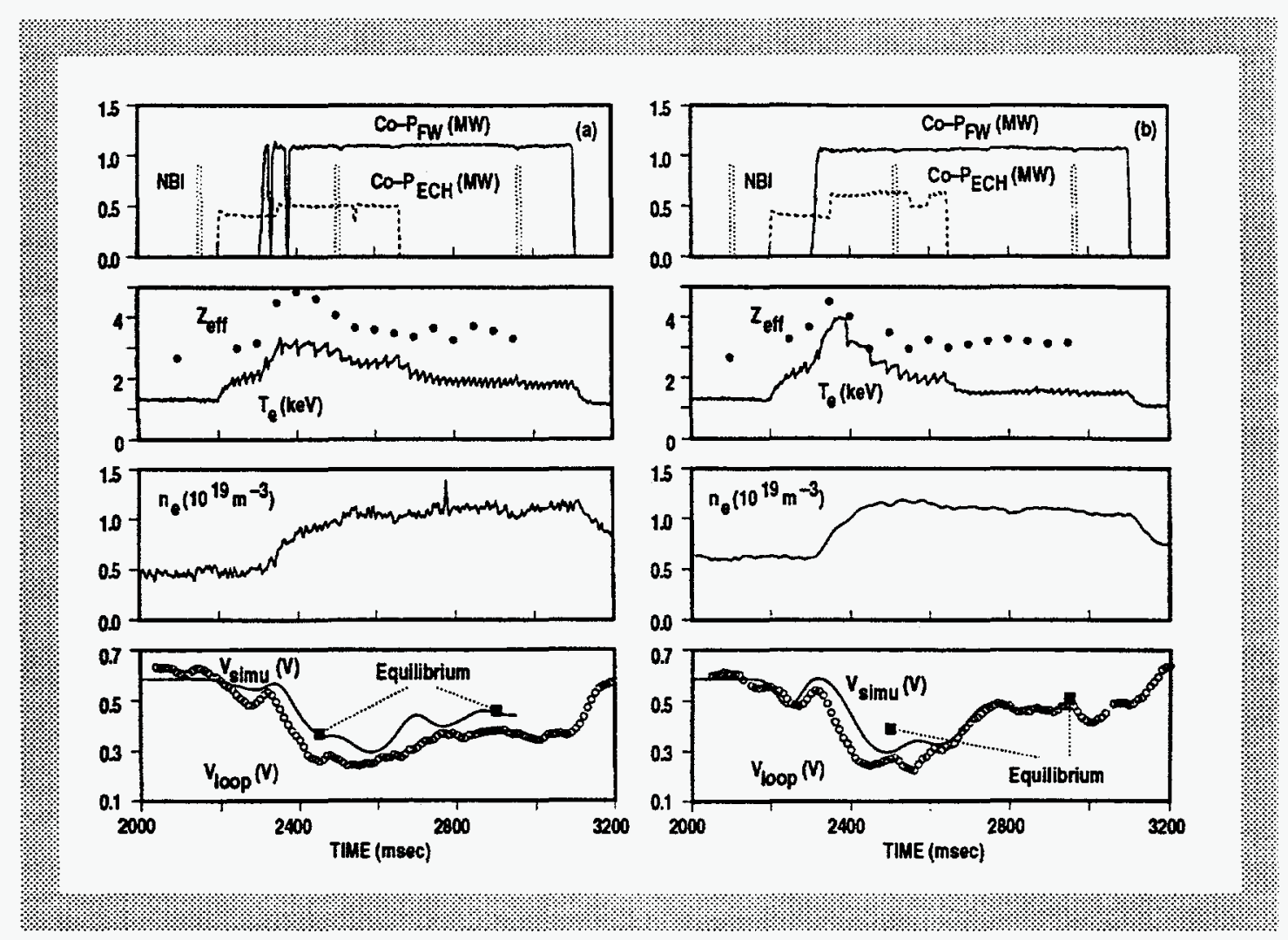

Fig. 3.5-1. (a) Behavior of an ECH-preheated discharge with co-FWCD. $B_{T}=1.1 T, I_{p}=0.4$ MA. Shown are coupled FW and ECH power, along with the timing of short diagnostic neutral beam pulses, the central electron temperature as measured by $2 \Omega_{e}$ electron cyclotron emission, the effective ion charge $Z$, the lineaveraged electron density, the measured surface voltage $(0)$, and the calculated surface voltage assuming no if current drive (solid line). Also shown is the "snapshot" calculation of the surface voltage (filled boxes). (b) Behavior of an ECH-preheated discharge similar to that of (a), except with counter-FWCD.

period. The rapid changes in central temperature cause the loop voltage at the surface not to be equal to the value near the magnetic axis, as is evident from the disagreement between the two methods of calculating the surface voltage in this phase of the discharge. When the timedependence is properly taken into account (solid curve), the net current drive in the combined $\mathrm{ECH}+\mathrm{FW}$ portion of the discharge is found to be $+35 \pm 35 \mathrm{kA}$, the slightly positive value presumably resulting from the co-ECCD; in the FW-only portion of the shot, no net current drive was observed.

Apart from the gross difference in the level of current drive, other, more subtle, differences between co- and counter-FW injection were observed. The difference in sawtooth behavior has already been mentioned. In the co-FWCD cases, the minor radius of the $q=1$ surface, determined from the soft $\mathrm{X}$-ray signals, increases with the level of driven current from about 
$12 \mathrm{~cm}$ in the ohmic discharge to $22 \mathrm{~cm}$ with $0.12 \mathrm{MA}$ of current drive. The behavior of the internal inductance $\ell_{\mathrm{i}}$ is also consistent with significant centrally-peaked current drive in the co-FWCD case and little or no counter-FWCD.

The level of co-FWCD is a strongly increasing function of FW power per particle. This is shown in Fig. 3.5-2, where the noninductive current drive (rf-driven plus neoclassical bootstrap current) expressed as a fraction of the total plasma current is plotted as a function of the FW power divided by the line-averaged density. Both discharges with and without $\mathrm{ECH}$ preheating are included. In the best case, the efficiency in the customary units is $\gamma \equiv \bar{n}_{\mathrm{e}} \mathrm{I}_{\mathrm{rf}} \mathrm{R} / \mathrm{P}_{\mathrm{FW}} \equiv 0.028 \times 10^{20} \mathrm{~A} / \mathrm{W} / \mathrm{m}^{2}$. If the bootstrap current of $43 \mathrm{kA}$ is subtracted

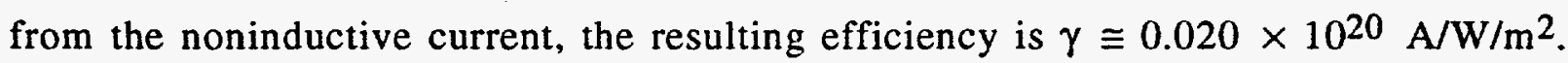

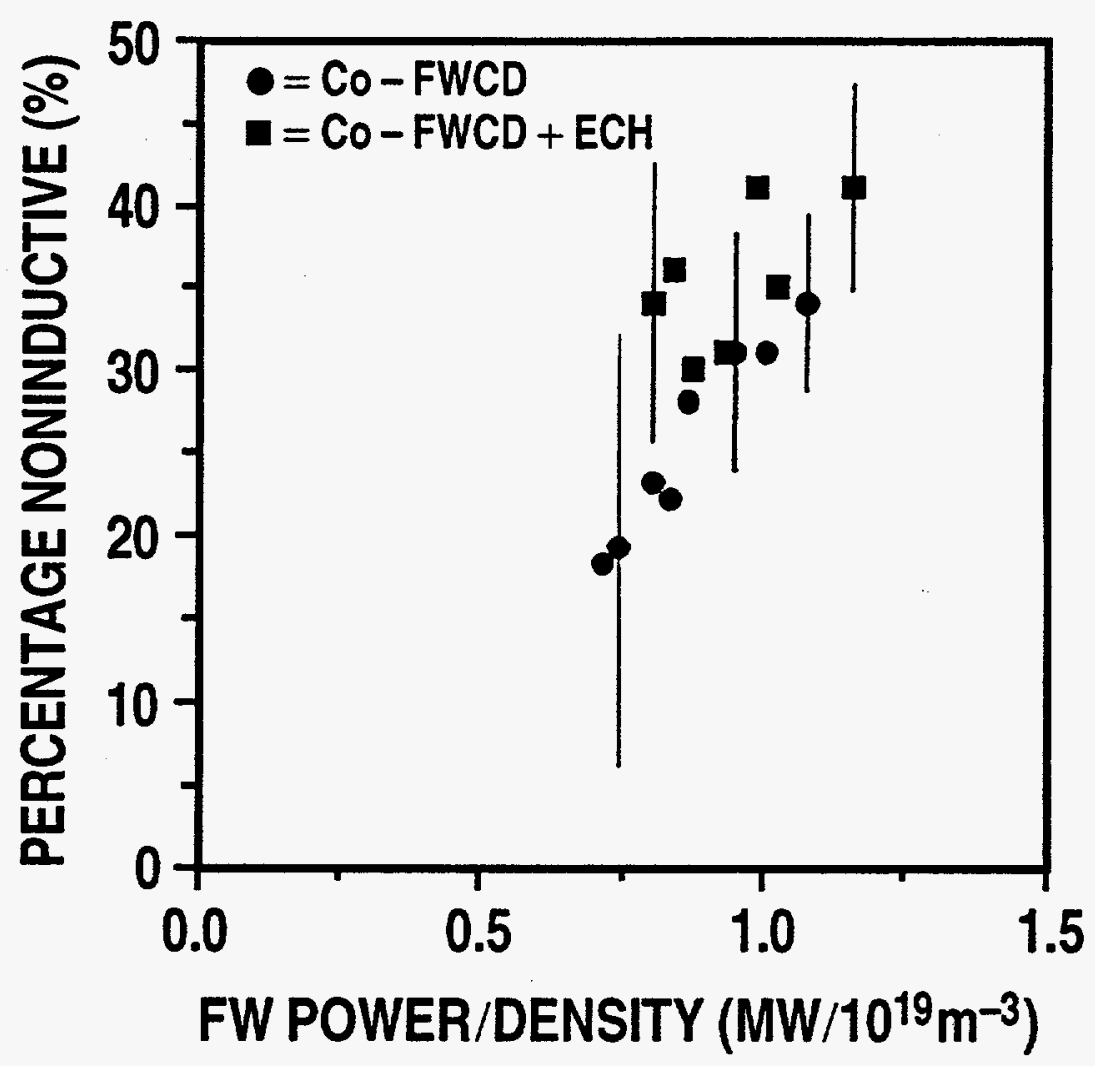

Fig. 3.5-2. Fraction of the total plasma current which is noninductively-driven as a function of the co-FWCD power level divided by line-averaged density. $\left(I_{P}=0.4 M A, B_{T}=1.1 \mathrm{~T}\right.$.) 
Increasing the distance between the antenna and the plasma surface by a factor of two appears to increase the current drive efficiency by about $40 \%$. The level of driven current is in approximate agreement with calculations based on multiple-pass ray-tracing using the measured profiles.

The current drive efficiency is evidently higher for the cases with ECH preheating than those with FW alone. This is primarily due to the higher central electron temperature obtained with preheating. Figure 3.5-3 shows that the current drive efficiency (not including the bootstrap contribution) increases with the central electron temperature for the same set of data in Fig. 3.5-2. The measured efficiency has substantial scatter, but it is not inconsistent with the scaling $\gamma \sim 0.4 \mathrm{~T}_{\mathrm{e}}(0) / 10 \mathrm{keV} /\left(2+\mathrm{Z}_{\mathrm{eff}}\right)$ developed for ITER. Future experiments at higher FW and electron preheating power levels should permit fully noninductive CD in DIII-D at the $0.4 \mathrm{MA}$ level at $\mathrm{n}_{\mathrm{e}} \sim 1 \times 10^{19} \mathrm{~m}^{-3}, \mathrm{P}_{\mathrm{FW}}=2.0 \mathrm{MW}$, and correspondingly larger effects on the current profile.

Fig. 3.5-3. Current drive efficiency $\gamma$ for the same discharges as in Fig. 3.5-2 as a function of central electron temperature. Also shown is the ITER Physics Design Guideline for the FWCD efficiency.

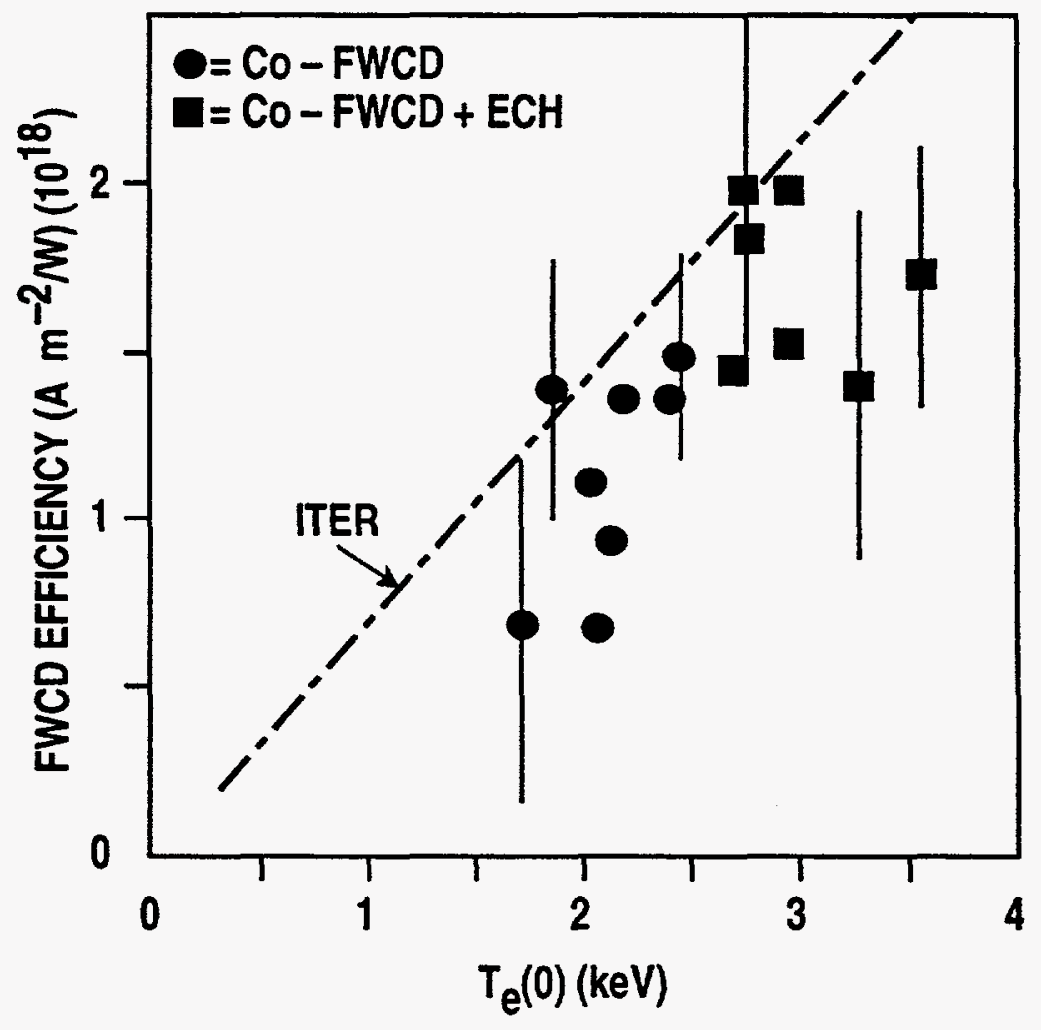


SECTION 4

\section{TOKAMAK PHYSICS}




\section{TOKAMAK PHYSICS}

\subsection{OVERVIEW}

This section describes the results of investigations into the general physics of tokamak plasmas with emphasis on the understanding of particle and energy transport in hightemperature tokamak plasmas and the parameter limits to plasma beta. Areas of special emphasis are: toroidal Alfvèn eigenmodes (TAE), the effect of plasma rotation on transport and stability, comparison of scaling laws between Joint European Tokamak (JET) and DIII-D, heat and particle pinches, and the effects of different types of auxiliary heating on plasma transport.

- Observation and analysis of TAEs.

- The observation of an inward electron "heat pinch" well outside the error bars in off axis electron cyclotron heating $(\mathrm{ECH})$ experiments. Similar results are obtained in off-axis neutral beam injection (NBI) heating experiments.

- The identification of gyro-Bohm-like scaling of the energy confinement time for ECH L-mode discharges.

- The demonstration of efficient direct electron heating by fast waves (FWs) at moderate power levels with the antenna Faraday shield removed.

\subsection{TOROIDAL ALFVÉN EIGENMODES AND OTHER GLOBAL ALFVÉN MODES}

Global Alfvèn modes such as the TAE are of considerable concern to fusion experiments since they can be driven unstable by energetic a particles if the $\alpha$ particles are in resonance $\left(v_{a} \geq v_{A}\right)$ and the energy imparted by the particles to the wave exceeds the energy loss through various damping mechanisms. In DIII-D, TAE modes are excited by simulating the $\alpha$ particles with energetic neutral beams [4.2-1]. The modes are exhibited as finite frequency magnetic 
perturbations on magnetic probes generally in the range 50 to $150 \mathrm{kHz}$ and with toroidal mode numbers in the range $2 \leq \mathrm{n} \leq 10$. The observed TAE modes are important because when the instability threshold is reached, they catastrophically expel the beam ions which causes a saturation in beam $\beta$ not much above the threshold. Beam losses of up to $70 \%$ have been observed in DIII-D [4.2-2].

Careful and detailed analysis of the 1991 experimental data, coupled with theoretical progress, has revealed a number of new and important results. The theoretical predictions and experimental observations of TAE modes have now been shown to be in good agreement [4.2-3,4.2-4]. Analysis of the data in one discharge has now confirmed the presence of the predicted [4.2-5] elongation-induced Alfvèn eigenmode (EAE) in DIII-D. Theoretical calculations have also predicted the existence of a new kind of global mode in finite $\beta$ discharges [4.2-6] — the beta-induced Alfvèn eigenmode (BAE) which, in addition to contributing to the observations at moderate $\beta$, also provides an explanation of the dependence of the observed mode frequencies at high $\beta$. Progress has also been made in the theory of continuum damping, in collaboration with groups at the University of Texas (Austin) and University of California at San Diego (UCSD), as well as in the theoretical description of the nonlinear cycle, in collaboration with the group at the University of California at Irvine (UCI).

The observed scaling of the TAE mode frequencies with the Alfvèn speed $v_{A}$ predicted by the simplest theories has now been shown to be in very good agreement with the more sophisticated predictions from ideal magnetohydrodynamic (MHD) calculations with the GATO code using the reconstructed experimental discharge equilibria. Two discharges were analyzed in detail, 71515 and 71524 . The computed and observed mode frequencies are shown in Fig. 4.2-1. For discharge 71524, three TAE modes were found in the calculations, two of which correspond closely with the two modes that were observed. The computed frequencies are shown in relation to the computed gap structure in Fig. 4.2-2. The computed modes at the higher frequencies in both 71515 and 71524 , which do not appear to correspond to observed TAE modes, are also found to be strongly coupled to the shear Alfvèn continuum near the edge (where they intersect the continuum as in Fig. 4.2-2). Consequently, they are expected to be more strongly damped, thus explaining why they are not observed.

Experimental observations are in good qualitative agreement with theoretical estimates of the stability of TAE modes. The fast particle destabilization and the various contributions to the damping rate were estimated for Discharge 71524 using recent published theories. 


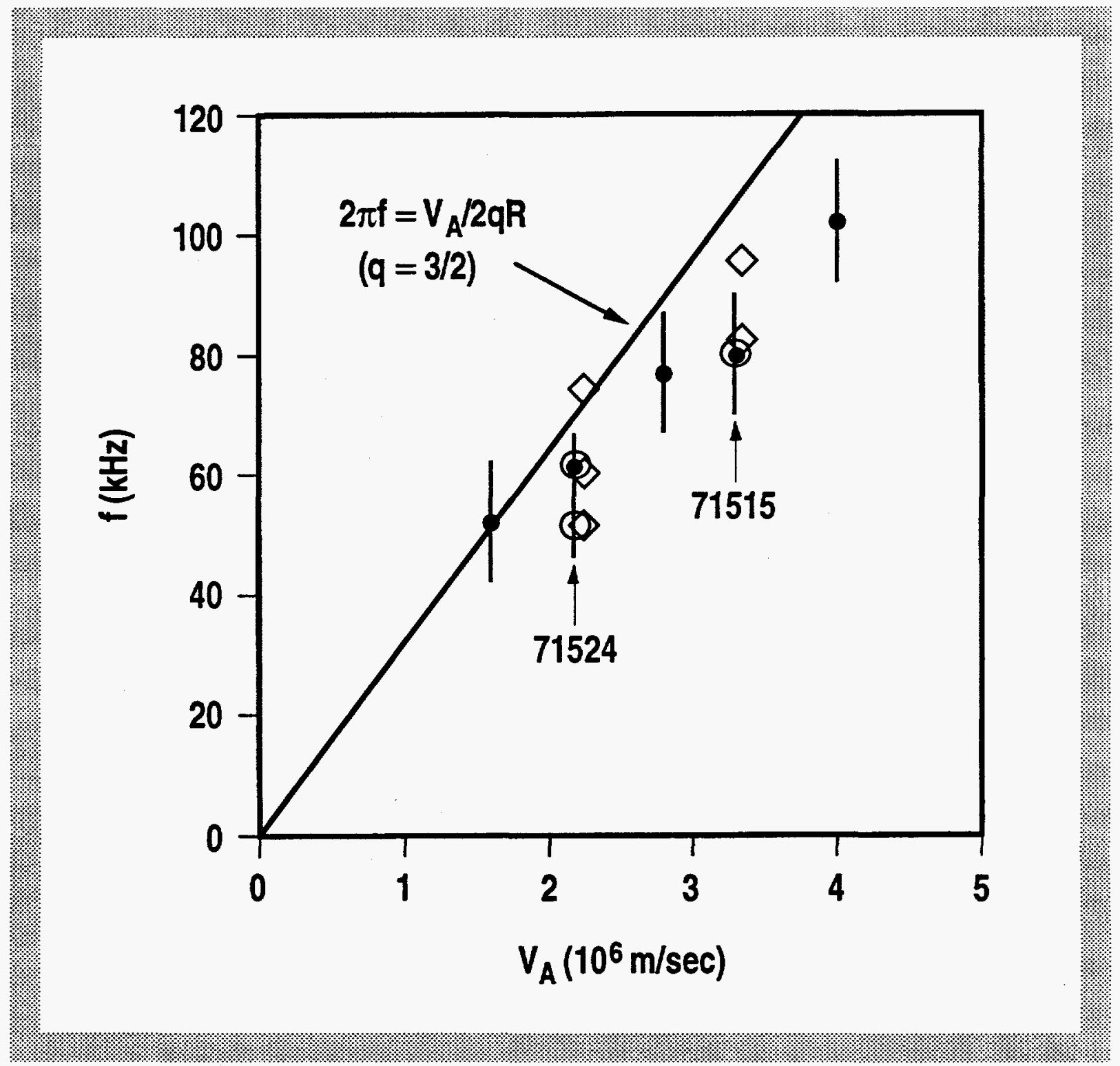

Fig. 4.2-1. TAE frequency dependence on $\mathrm{v}_{\mathrm{A}}$, observed (solid dots) and calculated (diamonds) frequencies at increasing toroidal magnetic field. More refined analysis of the experimental data is shown as the open circles.

Although the available theories make assumptions that are rather gross approximations for DIII-D (circular cross section, large aspect ratio, low $\beta$, for example), the estimates obtained provide an indication of the important physical trends and are actually in good qualitative agreement with the observed thresholds. The computed total growth rate is shown in Fig. 4.2-3 for varying toroidal mode number $n$. Discharge 71524 was close to 


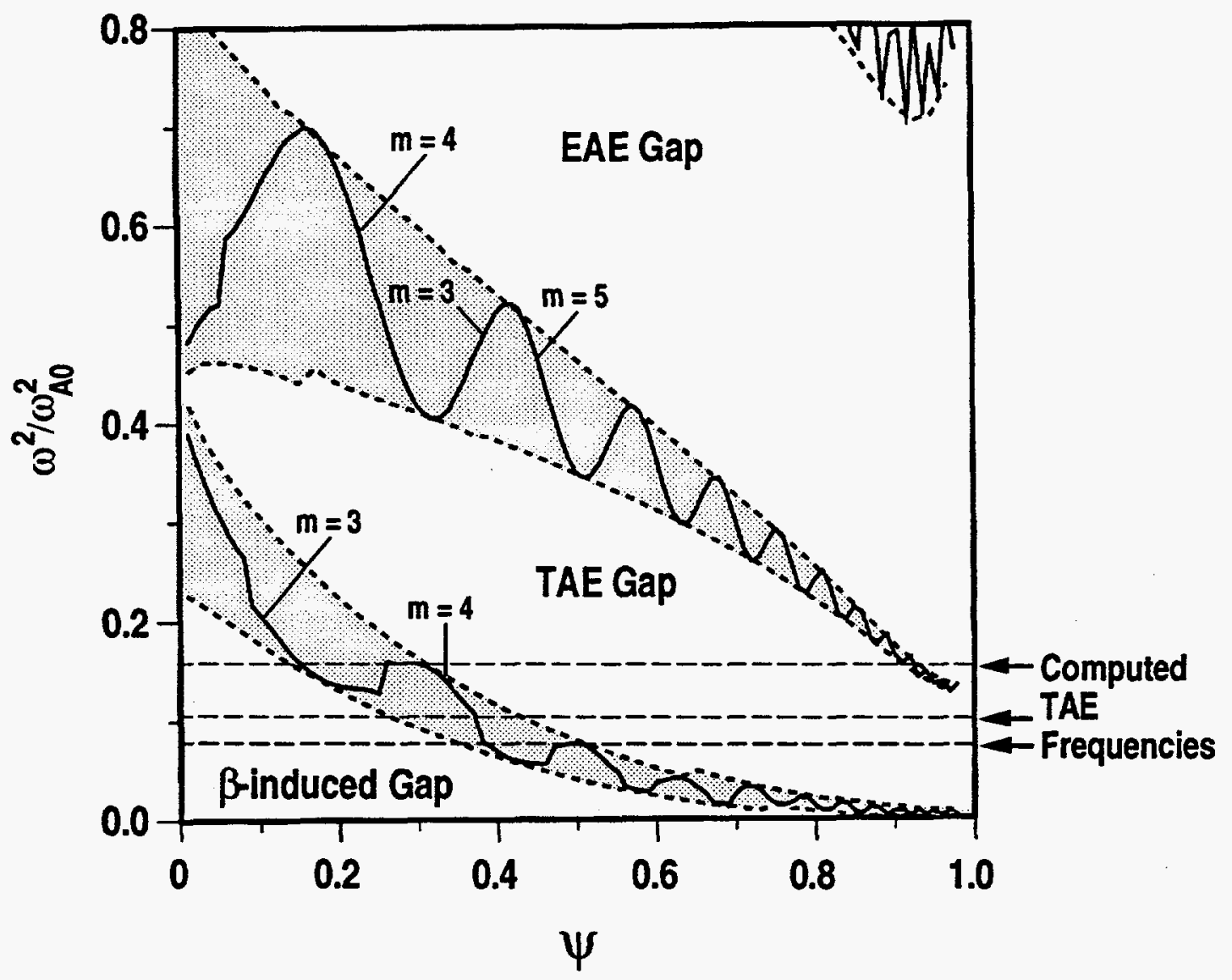

Fig. 4.2-2. Computed Altvèn spectrum for discharge 71524 at 1875 msec.

marginal stability and the computed fast particle destabilization and damping rates are found to be balanced within a factor of two; the damping rate is somewhat overestimated. The predicted most unstable mode numbers are $2 \leq \mathrm{n} \leq 5$ which coincides with the observed range of mode numbers. The upper cutoff in $\mathrm{n}$ is from electron kinetic damping (also called "radiative damping" as in Fig. 4.2-3). The calculations also predict that increased magnetic shear should preferentially stabilize lower $\mathbf{n}$ modes where the so-called continuum damping is dominant. This is confirmed in negative current ramp experiments; after the negative ramp the shear is high and only higher mode numbers are observed $(6 \leq n \leq 8)$, whereas later, after the current profile has relaxed and the shear is reduced, large amplitude, low 


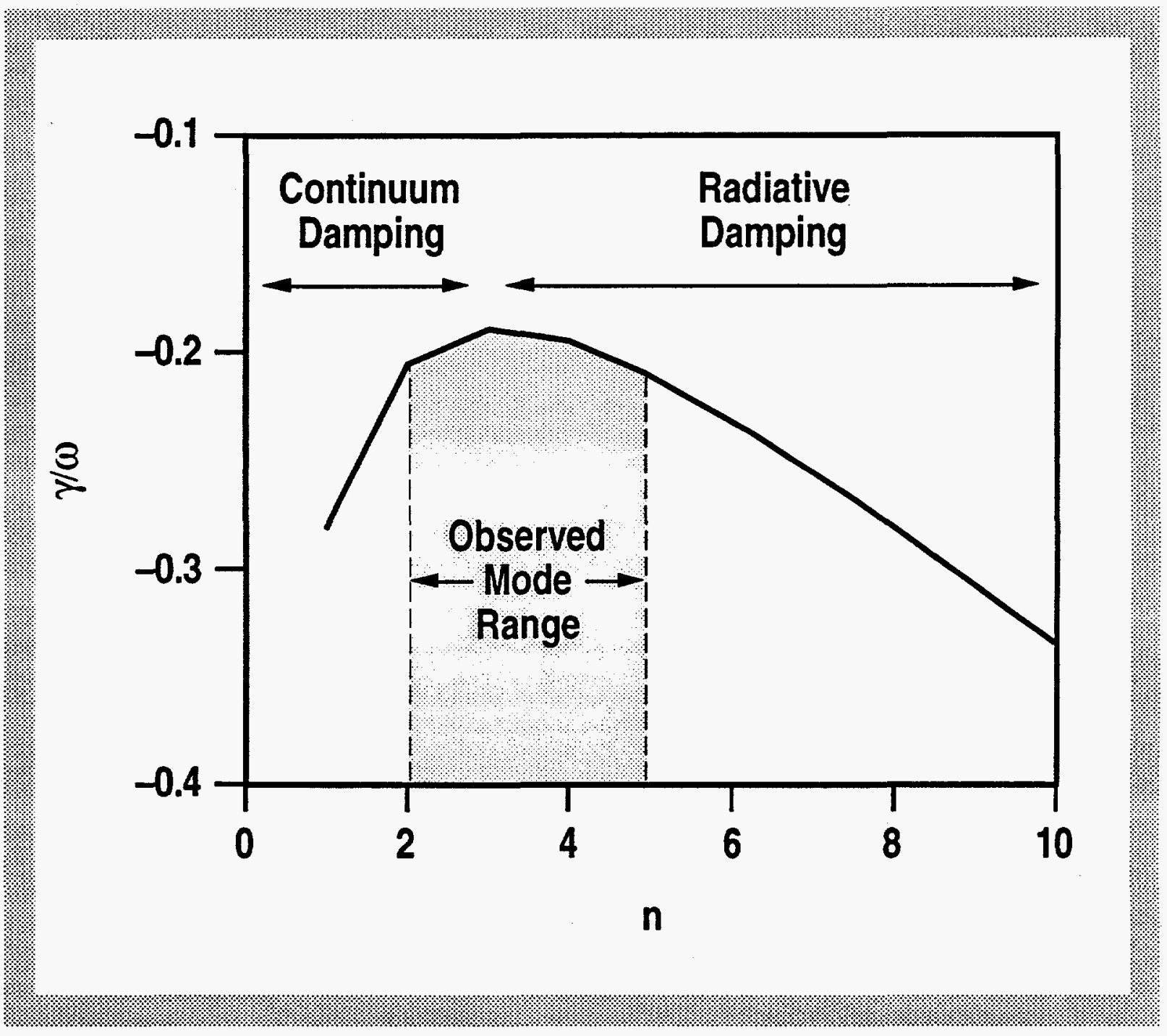

Fig. 4.2-3. Computed growth rate for discharge 71524 at 1875 msec as a function of toroidal mode number $n$.

mode numbers ( $3 \leq n \leq 5)$ are observed. Elongation is also found to suppress the low mode numbers, consistent with the observation that TAE modes in circular cross section the Princeton Tokamak Fusion Test Reactor (TFTR) discharges are generally lower $\mathrm{n}$ than in DIII-D. The underlying reason for this is not yet completely clear but one explanation is that noncircular shaping couples the TAE mode strongly to the continuum, thus enhancing the damping rate.

The existence of the predicted EAE mode has been confirmed in DIII-D experiments. The EAE mode is an isolated global mode created within a higher frequency elongation- 
induced gap from coupling of poloidal harmonics differing by two. In toroidally rotating DIII-D discharges, the expected EAE frequencies are typically above the bandwidth of the magnetic detectors. However, in discharge 75346, an $\mathrm{n}=1$ locked mode developed which reduced the Doppler shift enough so that high frequency EAE modes were observable. The power spectrum for this discharge is shown in Fig. 4.2-4. The lower frequency peaks centered around $120 \mathrm{kHz}$ correspond to TAE modes, whereas the peaks near $175 \mathrm{kHz}$ can be attributed to EAE modes. This is confirmed by comparing the observed mode frequencies with theoretical calculations of the gap structure for reconstructions of the discharge equilibrium. The TAE modes lie within the toroidicityinduced gap whereas the EAE mode frequency falls in the elongation-induced gap. Several magnetic probes with larger bandwidth have been installed in DIII-D which should permit more extensive studies of EAE modes in the future.

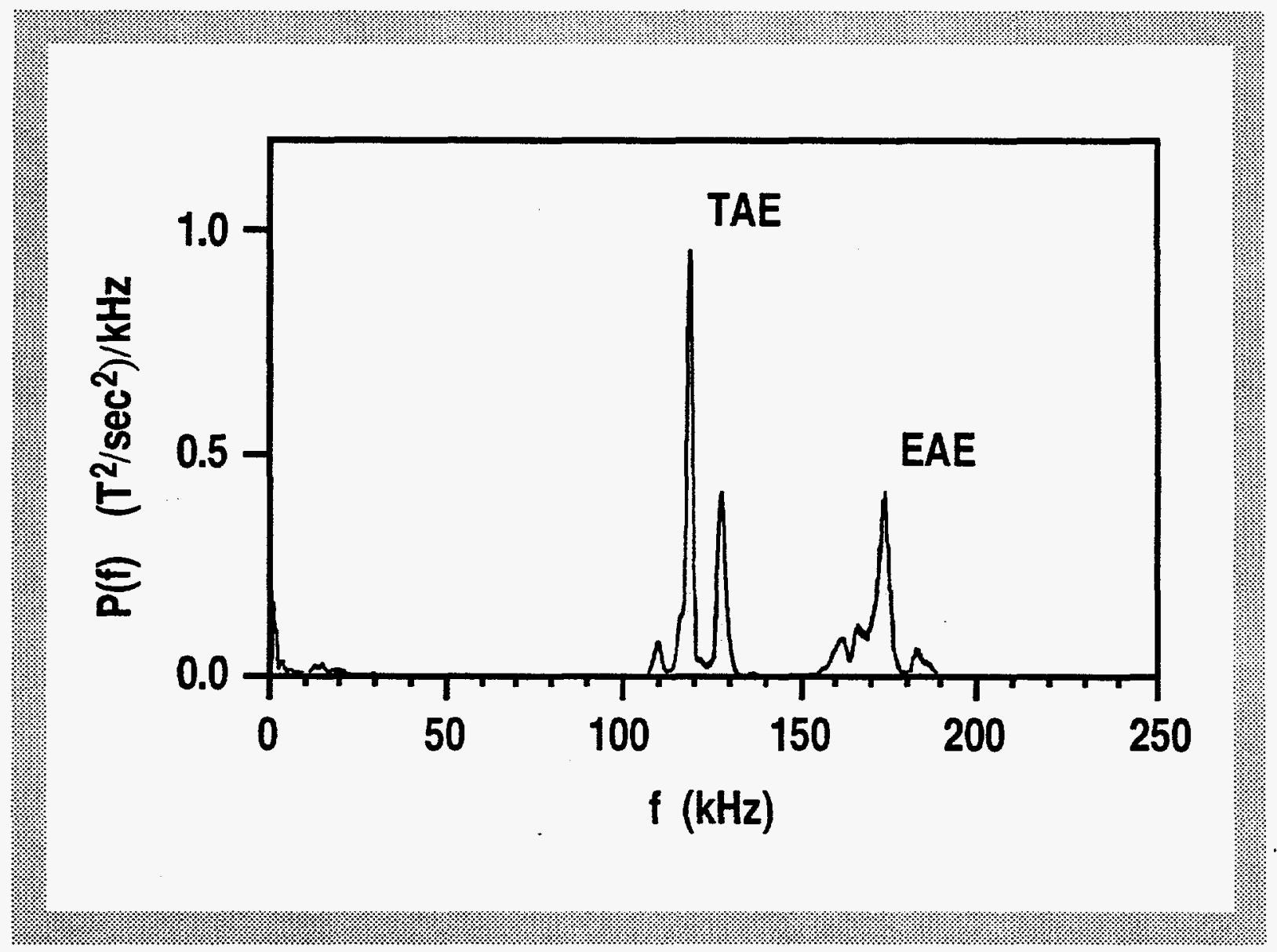

Fig. 4.2-4. Power spectrum for discharge 75346 showing TAE and EAE spectra. 
Calculations with the GATO stability code have predicted the existence of a new type of Alfvèn eigenmode in finite $\beta$ discharges. The TAE mode and gap structure calculations for discharge 71524 shown in Fig. 4.2-2 included coupling between the Alfvèn modes and the acoustic continuum through finite $\beta$ effects. This coupling creates [4.2-5] the $\beta$-induced gap shown in Fig. 4.2-2. The TAE mode calculations also revealed the presence of a new global mode in this gap - the BAE. This mode bears some similarity to the so-called global Alfvèn eigenmode (GAE) in cylindrical models but is a new mode since it and the associated gap are generated by acoustic coupling through finite $\beta$ and toroidicity. Typically, the TAE and BAE modes in DIII-D equilibria can exist in the same frequency range, with the BAE located inside the point where the continuum intersects the mode frequency as in Fig. 4.2-2, and the TAE located outside it. The mode displacements for two typical TAE and BAE modes corresponding to Fig. 4.2-3 are shown in Fig. 4.2-5.

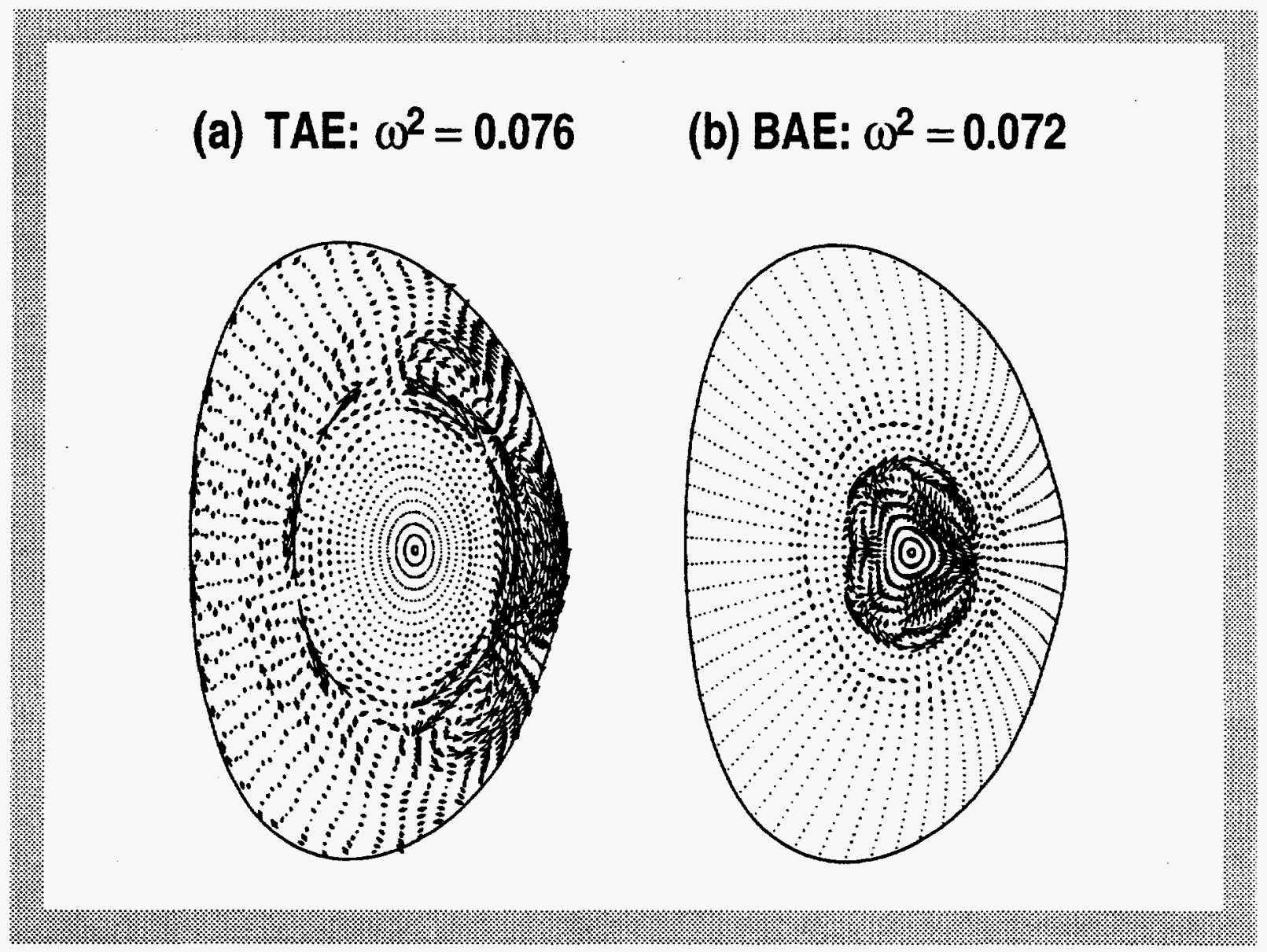

Fig. 4.2-5. Mode displacement plots for (a) TAE mode with $\omega^{2}=0.081$ and (b) BAE mode with $\omega^{2}=0.072$. 


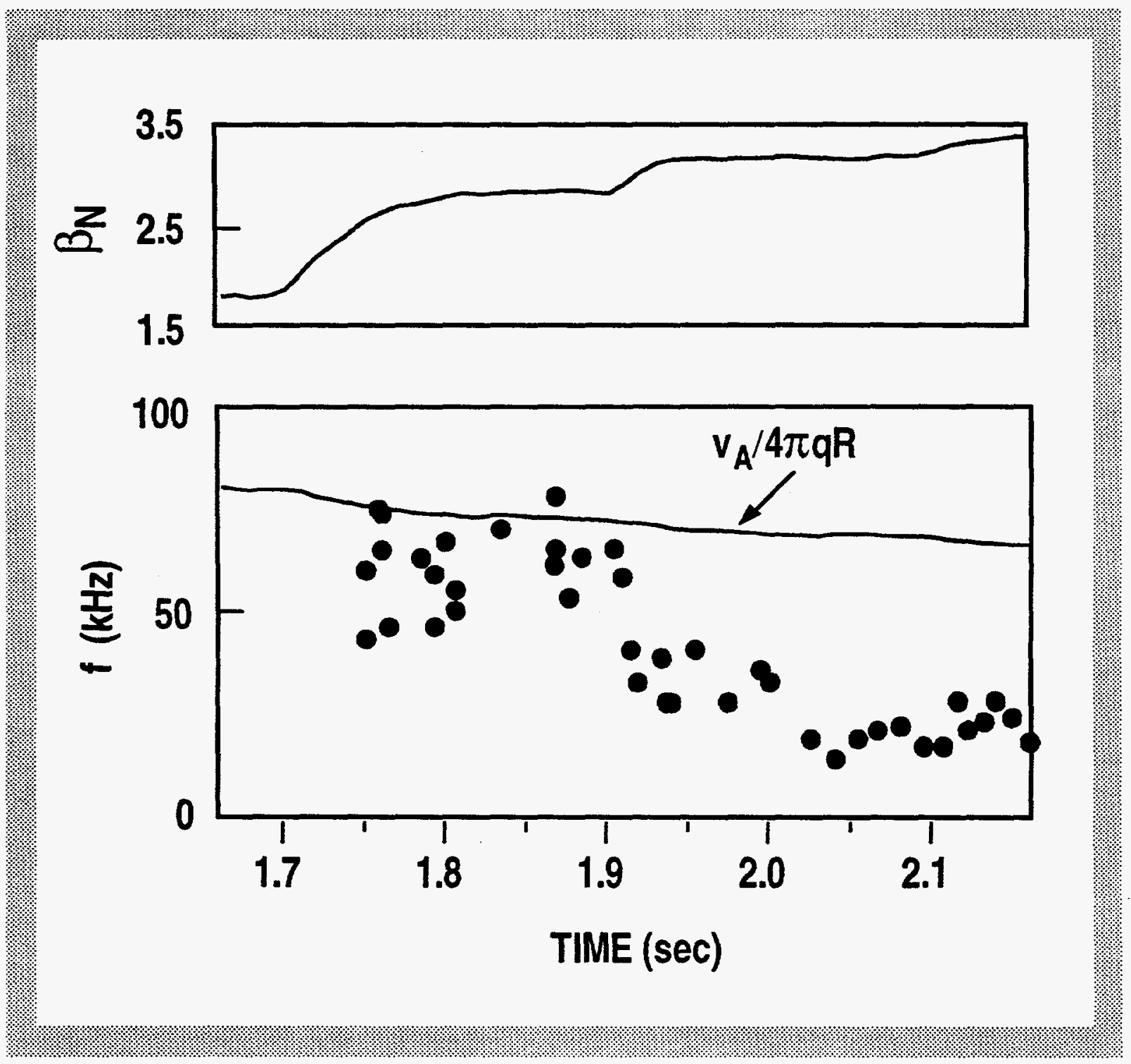

Fig. 4.2-6. Observed frequency dependence with increasing $\beta$ for discharge 71524 .

Experimental observations are consistent with the predicted BAE mode. As $\beta$ is increased experimentally, the observed mode frequency is found to decrease to about one third of the original TAE mode frequency (Fig. 4.2-6). The lower frequency modes at high $\beta$ also scale with the Alfvèn speed as shown in Fig. 4.2-7. However, if these low frequency modes correspond to TAE modes, they would have to be located outside the $\mathrm{q}=3$ surface where the beam drive is negligible. On the other hand, BAE modes are also present in this low frequency range and have their largest amplitude inside $\mathrm{q}=2$ where the beam drive is largest. The BAE 


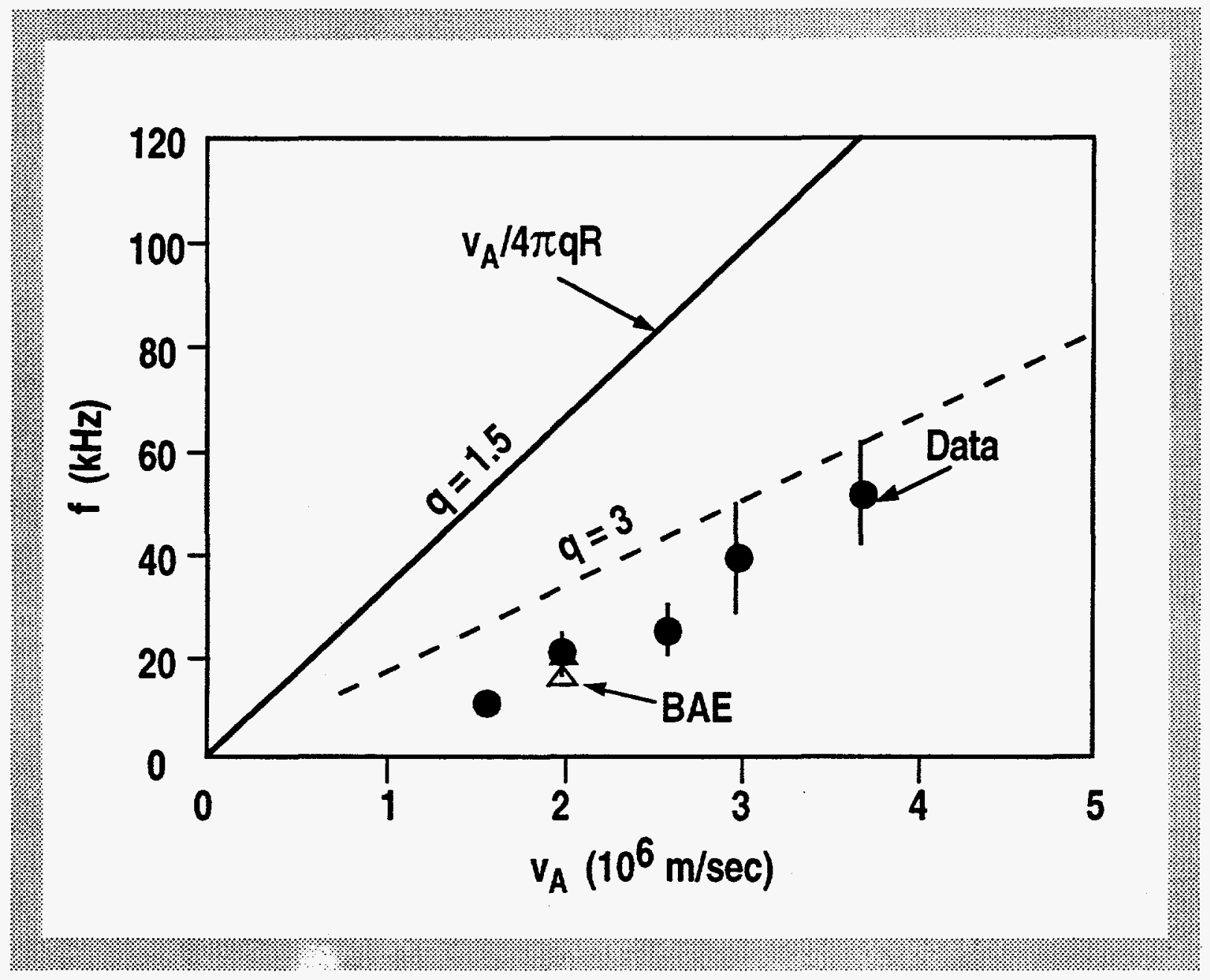

Fig. 4.2-7. Scaling of low frequency mode frequencies with Alfvèn speed $v_{A}$ showing computed BAE frequency for discharge 71524 at 2150 msec. The experimental data is shown as the solid dots, the GATO calculated BAE frequency is the open triangle and the predictions of the modes were kinetically destabilized ballooning modes (KBM) shown as $x$ 's.

and TAE modes can also be coupled which may also explain the fact that there is no obvious change in the characteristics of the observed modes beyond the decrease in frequency.

TAE modes cause large losses of energetic beam ions. Measurements of the $2.5 \mathrm{MeV}$ neutron emission produced by the deuterium beam ions indicate that up to $70 \%$ of the injected power is lost during strong TAE activity. Foil bolometers mounted on the wall of the vacuum vessel were used to corroborate the neutron measurements and to measure the poloidal distribution of the losses. The results (Fig. 4.2-8) indicate that the losses are concentrated near the outer midplane. At $10 \mathrm{~cm}$ outside the limiter surface 


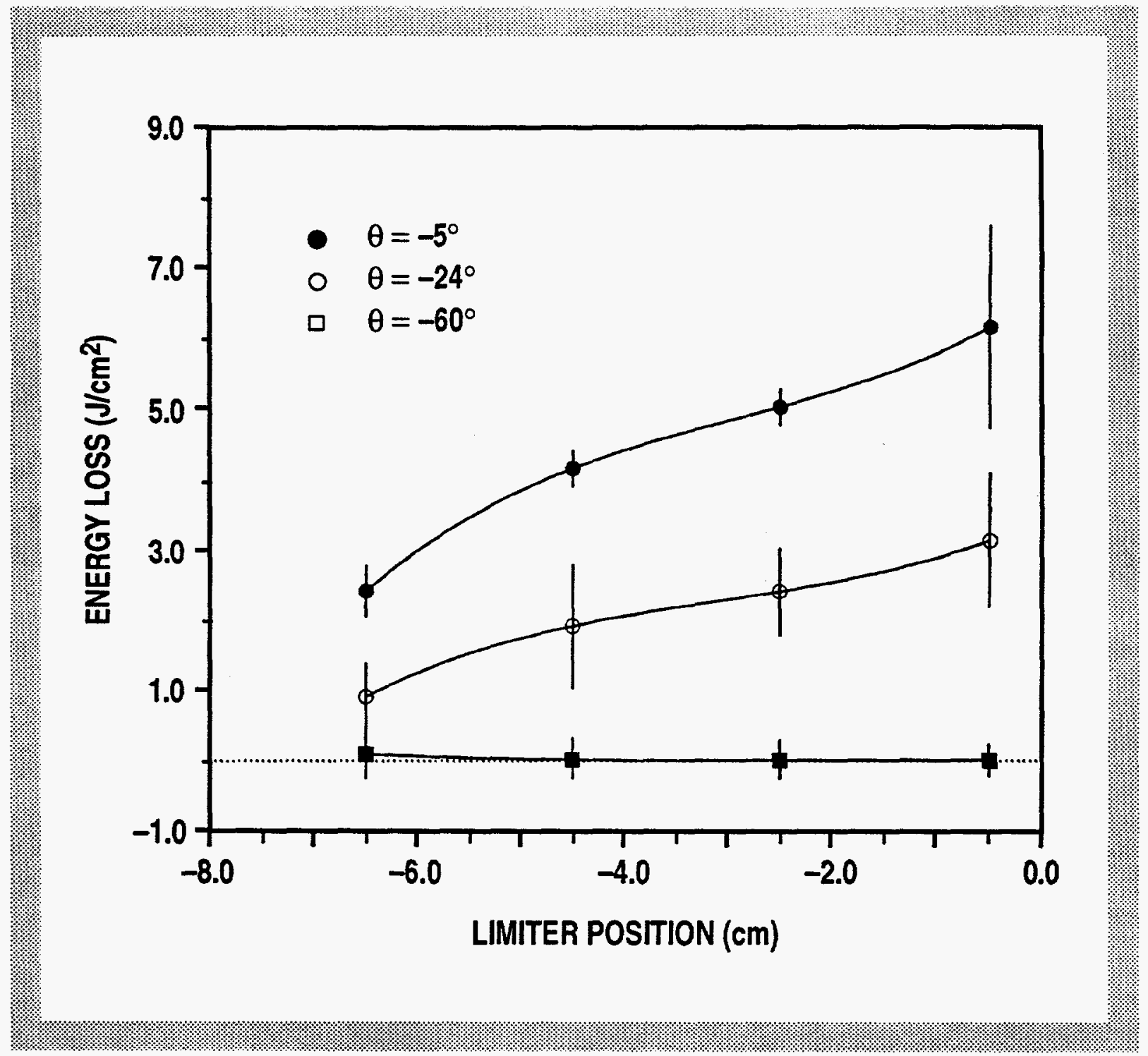

Fig. 4.2-8. Energy deposited in foil bolometers mounted on the vessel wall as a function of the position of an obstacle during TAE activity. The limiter on the opposite side of the machine from the foils was used as the obstacle and the measurements are relative to the first wall. The data are from three poloidal locations, with $-5^{\circ}$ near the midplane and $-60^{\circ}$ representing a foil below the midplane. The background plasma radiation is subtracted from the measured signal to show only the fast ion contribution.

Langmuir probe also detected the escaping heam ions and observed coherent modulation of the signal, suggesting that the fast ions are lost resonantly. An obstacle (the $180^{\circ}$ limiter) was inserted into the scrape-off region in order to obtain further information about the orbits of the escaping beam ions. The neutron, bolometer, and Langmuir probe 
data suggest that the majority of escaping beam ions spiral out of the machine due to a coherent $\mathrm{E} \times \mathrm{B}$ drift caused by the TAE instability.

The loss of beam ions controls the maximum amplitude of the TAE modes. The instability appears in bursts because the fast ions that drive the instability are ejected from the plasma when the amplitude of the mode becomes appreciable. The burst cycle is well described by a simple pair of coupled nonlinear equations. These equations successfully predict all of the gross features of the nonlinear cycle, including:

1. The clamping of the beam beta near the point of marginal stability.

2. The dependence of the fast-ion losses upon the rate of beam fueling.

3. The gradual increase in the period between bursts as the beam power increases.

4. The shortening of the duration of the burst as the beam power increases.

These findings imply that it will not be possible to operate a reactor above the marginal stability point of alpha-driven instabilities.

The global TAE mode can resonantly excite a localized Alfvèn wave in the continuum when their frequencies coincide. This results in phase mixing of the Alfvèn waves near the resonance which channels energy away from the TAE and contributes to its damping - the so-called continuum damping mentioned earlier. A new formulation of this damping process has been developed which is applicable, in principle, to general axisymmetric tokamak configurations, and which is amenable to numerical calculation in the general case. Numerical calculations for simple test cases reveal that the method gives excellent results. Application to more general equilibria is in progress.

The supporting theoretical work is being performed in close collaboration with several groups outside General Atomics (GA), namely at the University of California at San Diego (UCSD) (M.N. Rosenbluth and J. Candy), the University of Texas at Austin (H.L. Berk, R. Mett, and S. Mahajan), Oak Ridge National Laboratory (ORNL) (D. Spong), the CRPP in Lausanne, Switzerland (L. Villard) and at Massachusetts Institute of Technology (MIT) (C.T. Hsu). In the future, we anticipate that the various collaborative efforts will be expanded. 


\section{REFERENCES FOR SECTION 4.2}

[4.2-1] Heidbrink, W.W., et al., Nucl. Fusion 31 (1991) 1635.

[4.2-2] Duong, H.H., et al., "Loss of Beam Ions During TAE Instabilities," General Atomics Report GA-A21038, submitted to Nucl. Fusion; H.H. Duong, W.W. Heidbrink, "Confinement of Fusion-Produced MeV Ions in the DIII-D Tokamak," General Atomics Report GA-A20923, to be submitted to Nucl. Fusion.

[4.2-3] Strait, E.J., W.W. Heidbrink, M.S. Chu, et al., "Stability of TAE Modes in DIII-D," in Proc. 14th ICPP and CNFR (1993), General Atomics Report GA-A21045 (1992); E.J. Strait, W.W. Heidbrink, A.D. Turnbull, et al., "Stability of Neutral Beam-Driven TAE Modes in DIII-D," General Atomics Report, to be submitted to Nucl. Fusion.

[4.2-4] Turnbull, A.D., et al., "Global Alfvèn Modes: Theory and Experiment," General Atomics Report GA-A21138 (1992), submitted to Phys. Fluids B.

[4.2-5] Chu, M.S., et al., Phys. Fluids B4 (1992) 3713. 


\subsection{MAGNETIC BRAKING FOR STUDY OF THE ROLE OF ROTATION ON TURBULENCE AND CONFINEMENT}

The L-to-H transition is associated with a change in poloidal rotation at the edge of the plasma. The development of a radial gradient (shear) in $v_{\theta}$ acts to suppress microturbulence and transport by decorrelating fluctuations of the same frequency at different radii as the Doppler shift $\mathrm{k}_{\theta} \mathrm{v}_{\boldsymbol{\theta}}$ varies radially; $\mathrm{k}_{\theta}$ is the poloidal wave number. The fundamental quantity for shear stabilization of microturbulence is shear in velocity $v_{\perp}=\vec{E}_{\mathrm{r}} \times \overrightarrow{\mathrm{B}} / \overrightarrow{\mathrm{B}} \cdot \overrightarrow{\mathrm{B}}$ (not necessarily $\mathrm{v}_{\theta}$ where $\mathrm{E}_{\mathrm{r}}$ is the radial electric field and

$$
\mathrm{v}_{\perp} \approx \mathrm{E}_{\mathrm{r}} / \mathrm{B}_{\phi}=-\mathrm{v}_{\theta}+\mathrm{v}_{\phi}\left(\mathrm{B}_{\theta} / \mathrm{B}_{\phi}\right)+\partial \mathrm{P}_{\mathrm{i}} / \partial \mathrm{r} /\left(\mathrm{n}_{\mathrm{i}} \mathrm{Zie}_{\mathrm{B}_{\phi}}\right)
$$

where $B_{\theta}$ and $B_{\phi}$ are the poloidal and toroidal fields, $v_{\phi}$ is the toroidal plasma fluid velocity and the last term in Eq. (1) is the ion diamagnetic drift velocity. $E_{\mathrm{T}}$ shear stabilization is not necessarily an edge effect only. While the $v_{\theta}$ and $\partial \mathrm{P}_{\mathrm{i}} / \partial \mathrm{r}$ terms dominate at the edge $(\rho \geq 0.95)$ of $H$-mode plasmas, the $v_{\phi}\left(B_{\theta} / B_{\phi}\right)$ term dominates in the plasma core $(\rho \leq 0.9)$ where $\rho$ is the normalized minor radius.

We use "magnetic braking" of plasma rotation as an independent control of plasma rotation which does not change energy and momentum deposition from the neutral beams. Application of static, resonant low $\mathrm{m}, \mathrm{n}=1$ magnetic field perturbations from an external coil ("the $n=1$ coil") induces toroidally asymmetric eddy currents $(n=1)$ in the plasma at $\mathrm{q}=\mathrm{m} / \mathrm{n}=1,2$, and 3 which produce $\overline{\mathrm{J}} \times \overline{\mathrm{B}}$ forces which act to slow (brake) the plasma rotation. The magnetic braking greatly alters the core rotation and radial electric field. We then investigate the effects on turbulence and confinement.

The magnetic braking has little effect on poloidal rotation (in H-mode plasmas) but reduces toroidal rotation in the core to near zero from the initial $60 \mathrm{~km} / \mathrm{sec}$ rotation speed. As a result, the peak core radial field is reduced from $35 \mathrm{kV} / \mathrm{m}$ (peak $\mathrm{E}_{\mathrm{r}} / \mathrm{B}_{\phi}=30 \mathrm{~km} / \mathrm{sec}$ ) to near zero. The reversed $E_{r}$ and region of high $E_{r}$ shear at the edge $(\rho \geq 0.95)$ are unaffected. There is no significant change in plasma energy, confinement, internal inductance, density profile, electron temperature profile or ion temperature profile. The good confinement $\mathrm{H}$-mode is maintained by the high $\mathrm{E}_{\mathrm{T}}$ edge shear and transport barrier.

Measurements of microturbulence by far infrared (FIR) scattering which is focused at $\rho \approx 0.8$ and detects $\mathrm{k}_{\theta}=2 \sim 4.5 \mathrm{~cm}^{-1}$, show that the magnetic braking downshifts the 
frequency spectrum measured. This is consistent with the reduction in the Doppler shift $\mathrm{k}_{\theta} \mathrm{E}_{\tau} / \mathrm{B}_{\phi}$ which dominates the frequency spectrum. The integrated power (proportional to density fluctuation squared $\tilde{n}^{2}$ ) over positive frequency, i.e., the core, increases by about a factor of two. This is consistent with theoretical predictions that reducing $E_{\mathrm{T}}$ shear will increase turbulence levels. The lack of an effect on confinement can be attributed to the small density gradient in the $\mathrm{H}$-mode core so that if the fluctuations increase there is little density gradient to drive transport along. It is also possible that core transport is due to density turbulence at shorter or longer wavelengths than measured or to magnetic turbulence.

Magnetic braking is a useful new tool for evaluation of the relation between rotation, radial electric field shear, turbulence and confinement. It should prove valuable for the study of the causes of the $\mathrm{VH}$-mode and high $\ell_{\mathrm{i}}$ confinement improvements attained this past year.

\subsection{DIMENSIONLESS VARIABLE SCALING EXPERIMENTS}

Empirical scaling laws for confinement based on statistical data have traditionally been expressed in terms of machine variables. For example ITER-89P L-mode scaling is $\tau \propto \mathrm{I}^{0.85} \mathrm{~B} 0.2 \mathrm{P}-0.5 \mathrm{n}^{0.1} \mathrm{R}^{1.2} \mathrm{a}^{0.3}(\mathrm{~b} / \mathrm{a})^{0.5}$. However to reveal the mechanism causing confinement loss, scaling laws can be re-expressed in terms of global average dimensionless plasma physics variables such as the relative gyroradius $\left(\rho^{*}=\rho / a\right)$, plasma beta $(\beta)$, and collisionality $\mathrm{V}^{*}$. Normalizing the confinement time $\tau$ to the cyclotron frequency $\Omega$, this scaling law can be re-expressed as $\tau \propto \Omega^{-1} \rho^{*-2.05} \beta-0.525 v^{*-0.075} a^{0.075} \mathrm{~F}(\mathrm{q}, \mathrm{R} / \mathrm{a}, \mathrm{b} / \mathrm{a})$ where $F$ is some power law expression and the small dimensional dependence $\mathrm{a}^{0.075}$ indicates some deviation from the constraints of plasma physics. The dependence on $\rho^{*}$ suggests that the macroscopic eddies scaling with the system size are most important ( $\tau \propto \Omega^{-1} p^{*-2}$ or Bohm-like scaling) in contrast to microscopic eddies scaling with the gyroradius (gyroBohm-like scaling $\tau \propto \Omega^{-1} \rho^{*-3}$ ). The unfavorable scaling with $\beta$ suggests an electromagnetic mechanism and the weak dependence on $v^{*}$ suggests a collisionless mechanism.

DIII-D has continued a program begun in 1990 to determine if these dependencies of global confinement scaling are true indicators of the confinement mechanism. Most puzzling is the dependence on $\rho^{*}$, since all theories indicate a gyroBohm-like rather than Bohm-like mechanism. Attempts were made to create dimensionally similar discharges 
GA-A21186; FY92 DIII-D Research Operations Annual Report

which varied only $\rho^{*}$. The first experiments [4.4-1] suggested a local effective diffusivity with gyroBohm. The Bohm scaling of total confinement was blamed on variations in the heating profile. Later experiments [4.4-2] with vertically shifted plasmas keeping the heating profile fixed showed that the local effective diffusion coefficient itself was more dependent on the heating profile than changes in $\rho^{*}$. This seemed to indicate that the beam heated discharges were operating near a critical temperature gradient and, in fact, both gyroBohmlike and Bohm-like diffusion models with critical temperature gradients could equally well fit the discharges even though the global confinement remained Bohm-like in all cases [4.4-3].

During FY92, these $\rho^{*}$-scaling experiments continued in two directions. First, attempts were made to compare dimensionally similar JET and DIII-D discharges. This allowed much larger variations in $\rho^{*}$. Achieving good similarity conditions with one machine has proven to be difficult, and the initial results of a JET/DIII-D comparison proved to be unsatisfactory [4.4-4]. An overriding problem has been the relatively high $Z_{\text {eff }}$ and proton defect for JET compared to DIII-D making the match of dimensionless parameter almost impossible. Although the scaling of global confinement remained Bohm-like, no real conclusion can be drawn until cleaner JET discharges are found.

Secondly, experiments were planned to make dimensionally-similar discharges with of strong central heating, which would stay well away from any critical temperature gradient. In preparation, two such discharges with exceptionally good similarity conditions were found in the existing database. Figure 4.4-1 shows that a ratio of effective diffusivity had a gyroBohm scaling and the global confinement scaling was slightly better than gyroBohm. While these discharges had only a small ohmic heating contribution, they were in a low density regime not far removed from the neo-Alcator scaling regime. Since the later scaling is known to be gyroBohm-like, it is not yet clear if there is a change of mechanism from gyroBohm to Bohm in going deeper into L-mode or if gyroBohm global scaling always results with central heating. Further experiments at higher density are planned for FY93.

First attempts at a controlled $\beta$-scaling experiment were made in FY92. Since most tokamak statistical databases have a collinearity between variations in $\rho^{*}$ and $\beta$, it has been suggested that the unfavorable $\beta$-scaling was a statistical tradeoff for lack of the expected favorable gyroBohm scaling in $\rho^{*}$. Most theoretical models suggest electrostatic processes dominate for at least $\beta<1 / 2 \beta_{\text {crit }}$. Two sets of discharges were made varying only $\beta$ at low $\beta\left(1 / 8 \beta_{\text {crit }}<\beta<1 / 4 \beta_{\text {crit }}\right)$ and moderate $\beta\left(1 / 4 \beta_{\text {crit }}<\beta<1 / 2 \beta_{\text {crit }}\right)$. Preliminary 
Fig. 4.4-1. Ratio of $1.99 \mathrm{~T}$ and $1.07 \mathrm{~T}$ onefluid heat diffusivity versus normalized radius (filled squares).

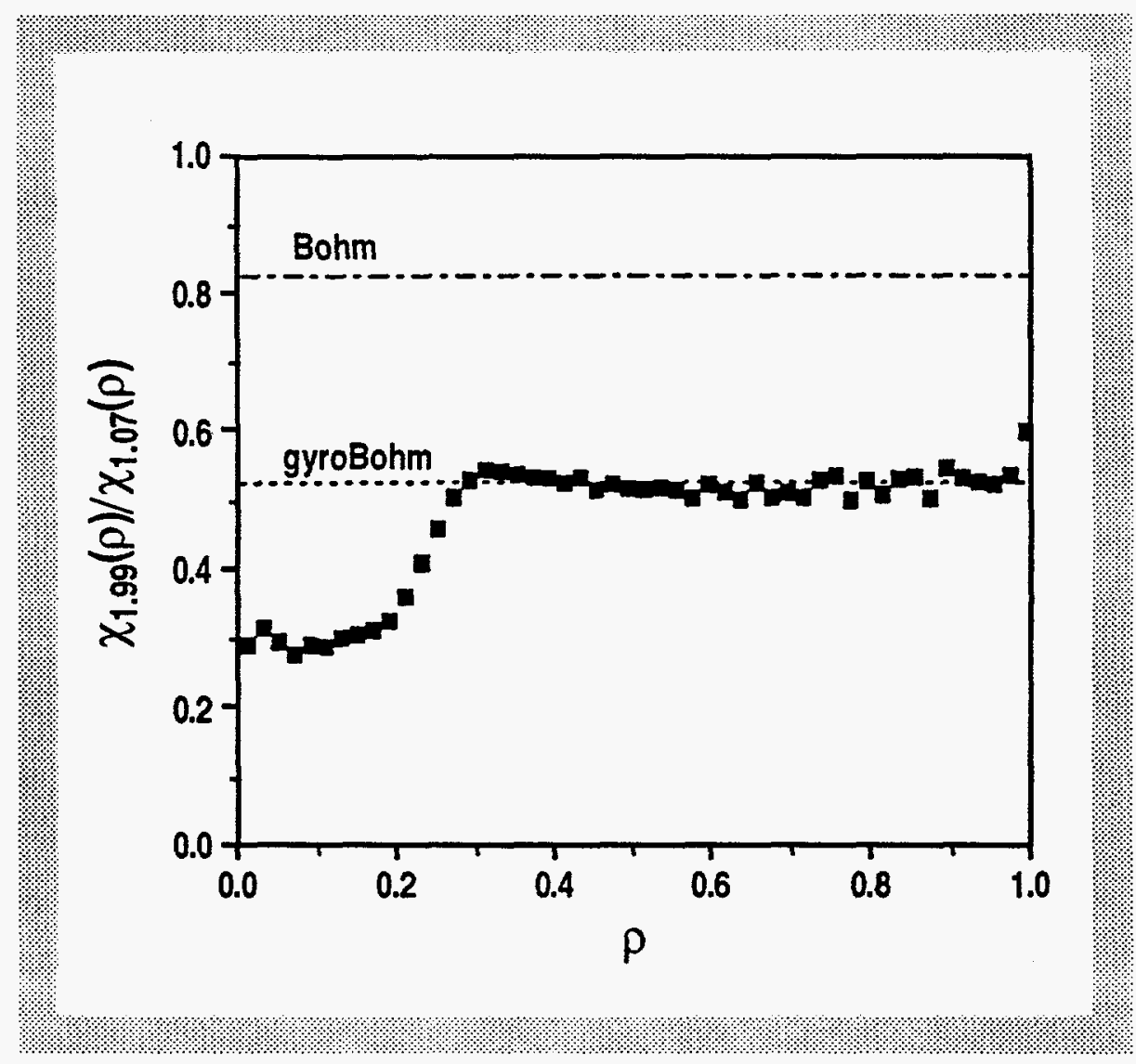

analysis of the global scaling based on magnetic data appear to show little dependence on $\beta$ which is a surprising deviation from the standard empirical scaling. Further analysis is in progress, but problems with radiation blinding of the profile diagnostics may require the experiments to be repeated.

\section{REFERENCES FOR SECTION 4.4}

[4.4-1] Waltz, R.E., J.C. DeBoo, and M.N. Rosenbluth, Phys. Rev. Lett. 65 (1990) 2390.

[4.4-2] DeBoo, J.C., R.E. Waltz, T.H. Osborne, in Proc. 18th EPS Conf. on Controlled Nuclear Fusion and Plasma Physics, June 3-7, 1991, Berlin, Germany, Vol. 15C, Part I (1991) 173; General Atomics Report GA-A20466 (1991).

[4.4-3] Waltz, R.E., J.C. DeDoo, T. H. Osborne, Nucl. Fusion 32 (1992) 1051. 


\subsection{CONFINEMENT SCALING WITH ELECTRON CYCLOTRON HEATING}

The study of confinement scaling with ECH is particularly attractive because the heating profile can be held constant over a wide range of parameters, unlike neutral beam heating. A database of approximately $250 \mathrm{~L}$-mode discharges with $\mathrm{ECH}$ as the only auxiliary heating source has been compared with a number of scaling laws. The data are all for deuterium plasmas with little variation in $\mathrm{R}$ or minor radius a. Both outside launch $\mathrm{O}$-mode and inside launch $\mathrm{X}$-mode data are included, since a direct comparison shows no difference in confinement between these two scenarios. Only shots in which ECH dominates the remaining ohmic power are included in the fit. The total input power $\mathrm{P}$, current $\mathrm{I}$, lineaveraged density $\bar{n}$, vacuum toroidal field $B$, and the elongation of the $95 \%$ flux surface are the variables.

The empirical scaling laws which best predict the ECH confinement times are the ITER-89P and the Rebut-Lallia with an ad hoc $\mathrm{Z}_{\mathrm{eff}}$ model. However, in both cases, the data indicates that one or more of the exponents in the empirical scaling is incorrect for this database. A regression fit to the $\mathrm{ECH}$ database gives

$$
\tau_{\mathrm{E}}=0.0710 .56 \overline{\mathrm{n}}^{0.28} \mathrm{~B}^{0.89} \kappa^{0.91 / \mathrm{P}^{0.61}} .
$$

The correlation matrix shows that $\mathrm{P}, \mathrm{I}$, and $\overline{\mathrm{n}}$ are correlated in the database, so great care must be exercised in interpreting the scaling with these parameters. The main result of the scaling study is that a near linear scaling with toroidal field is indicated. In order to validate this scaling, a reduced data set with on-axis heating and similar elongation $(\kappa>1.5)$ and density $\left(\bar{n}<2.0 \times 10^{13} \mathrm{~cm}^{-3}\right)$ was formed. Figure $4.5-1$ shows the energy confinement time corrected for the "conventional" I/P1/2 scaling plotted against $\mathrm{B}$. There is an obvious upward trend with $\mathrm{B}$.

The conclusion is that there is a nearly linear scaling of confinement with B. This is consistent with the prediction of gyro-reduced Bohm scaling. Virtually all theoretical models for plasma transport can be put into this gyro-reduced Bohm form while none of the empirical scaling laws for $\tau_{E}$ have such a dependence. It is not clear why the B scaling appears in the $\mathrm{ECH}$ database and not in the empirical scalings. One possibility is that the heating in the $\mathrm{ECH}$ database is central electron heating while the other databases are dominated by neutral beam heating where the power is split between electrons and ions. Another possibility is that these other databases are dominated by low q discharges whereas the ECH database has mainly discharges with $\mathrm{q}>4$. 
Fig. 4.5-1. Energy confinement time for fundamental $(2 T)$ and second harmonic (1 T) ECH, corrected for the "conventional" I/P1/2 scaling.

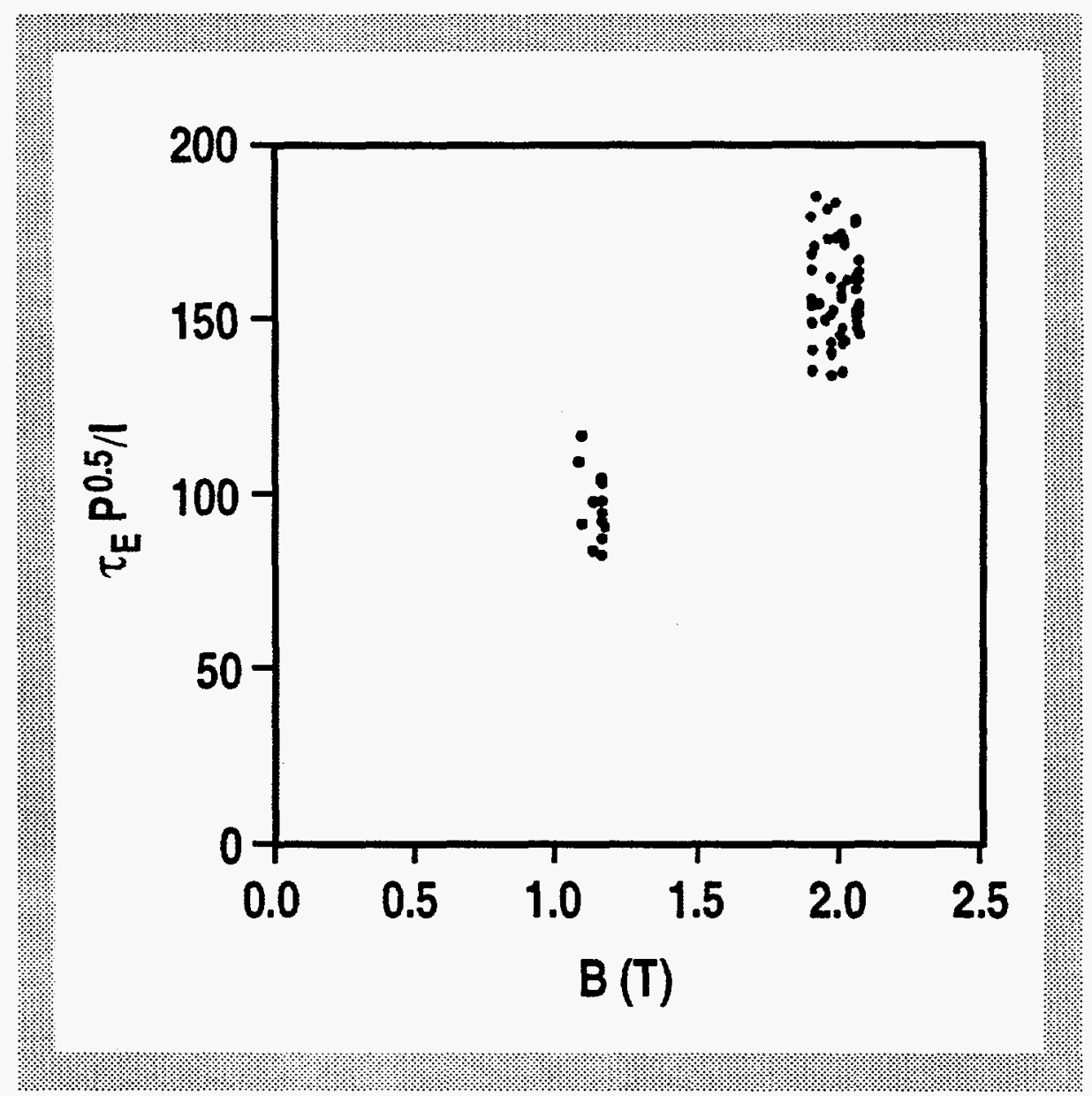

\subsection{THEORY OF PLASMA PARTICLE AND HEAT PINCHES}

The motivation for studying plasma particle pinches comes from the long-known experimental fact that moderate to high density tokamaks are capable of maintaining peaked density profiles in the central plasma region where there are no significant particle sources. Interest in heat pinches has a more recent origin and has been significantly driven by experimental results from the DIII-D tokamak. There is experimental evidence from DIII-D that both electron [4.6-1] and ion [4.6-2] heat pinches can occur; the evidence for the electron heat pinch inferred from ECH experiments, seems particularly compelling at this point, while the evidence for an ion heat pinch coming from off-axis heating NBI experiments is less clear. It is important to realize that the physical mechanisms underlying the heat pinches for each species can differ. 
The important new results summarized here are that the drift wave anomalous transport model can provide an explanation for the existence of both plasma particle and electron and ion heat pinches. This explanation is particularly appealing because the underlying cause of the particle and heat pinches is the presence of electrostatic ion temperature gradient (ITG) mode turbulence, which is also widely believed to be the dominant loss mechanism in the bulk plasma region of a hot tokamak. These conclusions were obtained from extensive numerical work using a simplified sheared magnetic slab model which neglects the effect of linear toroidal mode-coupling that is found in a torus. Accordingly, analogous calculations must be performed in toroidal geometry to validate the present results; some preliminary work in this direction has shown both qualitative and quantitative agreement.

The particle pinch mediated by ITG mode turbulence is predicted from the simplest quasilinear formalism. Previously, self-consistent, normal-mode solutions were not employed in evaluating the quasilinear particle flux and erroneous conclusions were obtained [4.6-3]. A correct evaluation shows that a particle pinch can occur over a wide range of the electron collisionality parameter $v^{*}, 0.2 \leq v^{*} \leq 1.0$, for the experimentally relevant range of $\eta_{e}=\partial \ell_{N} T_{e} / \partial \ell_{N} N_{e}, \eta_{e}=2$ to 3 . Another interesting feature of the particle pinch is that the role of circulating electrons is surprisingly large, generally greater than that of the trapped electrons, a result that is opposite to the "conventional wisdom" in the area of drift wave stability and transport. The justification for the neglect of circulating electrons in previous drift wave stability and transport analyses has almost always been based on simple intuitive arguments and anecdotal information. At the same time, it should be stressed that the importance of the circulating electrons may be peculiar to the slab model because of the small radial width of the ITG mode eigenfunctions; in toroidal geometry the radial mode widths are generally larger.

The strict quasilinear formalism also predicts an ion heat pinch driven by ITG mode turbulence in the range of typical experimental parameters. In this case, an additional feature is added to the sheared magnetic slab model, a light impurity ion species (in the present case, carbon). Impurity ion effects have been recently suggested as a source of isotope scaling in the energy confinement time [4.6-4]. Under certain conditions, impurities can also cause inward primary ion heat flow. Specifically, quasilinear flow calculations show that for $\mathrm{Z}_{\text {eff }}$ profiles that are nearly flat (or slightly hollow) and of moderately low value $\left(Z_{\text {eff }} \leq 2\right)$ a strong primary ion heat pinch occurs. There appears to be a systematic 
variation of the $Z_{\text {eff }}$ profiles between on- and off-axis NBI heating in DIII-D that is consistent with the theoretical predictions for the occurrence of an ion heat pinch, but unfortunately, the $Z_{\text {eff }}$ profile information is not complete so that a definitive conclusion cannot be made.

Finally, let us consider the electron heat pinch. The simplest version of quasilinear theory (with no impurity ion effects) predicts an electron heat pinch driven by ITG mode turbulence, but only at experimentally uninteresting values of $\eta_{e}, \eta_{e}$ « 1 . Motivated by the observation that circulating electrons were crucial to the particle pinch, the circulating electron response for both stability and quasilinear flows, was modified to include turbulent effects, i.e., "resonance broadening," due to a spectrum of ITG mode fluctuations. In a sheared magnetic slab, nonlinearity in the circulating electron response is manifest in two ways. Firstly, there is an $\mathrm{E} \times \mathrm{B}$ fluctuating drift nonlinearity that is also found in a twodimensional shear-free model. A second nonlinearity, that only occurs for nonzero magnetic shear couples fluctuating $\mathrm{E} \times \mathrm{B}$ drifts and parallel motion. The net effect of these two processes is to generate either an effective nonlinear damping or driving mechanism which acts on the circulating electron population. The new feature found here is the possibility of a nonlinear driving mechanism. It is this case that leads to an electron heat pinch (with no particle flow) for reasonable $\eta_{\mathrm{e}}, \eta_{\mathrm{e}} \simeq 1$. This prediction for $\eta_{\mathrm{e}}$ is below the range observed in the ECH experiments, $2 \leq \eta_{\mathrm{e}} \leq 3$, but the qualitative features of the calculation seem to be right. Note that a signature of the theoretical prediction for the electron heat pinch is a relatively small $\eta_{\mathrm{e}}$; if this is correct, then peaking the density profile in an NBI-heated plasma (at fixed electron temperature) should induce an electron heat pinch.

\section{REFERENCES FOR SECTION 4.6}

[4.6-1] Luce, T.C., C.C. Petty, and J.C.M. deHaas, Phys. Rev. Lett. 68 (1992) 52.

[4.6-2] Schissel, D.P., et al., Nucl. Fusion 32 (1992) 689.

[4.6-3] Waltz, R.E., and R.R. Dominguez, Phys. Fluids B 1 (1989) 1935.

[4.6-4] Dominguez, R.R., Nucl. Fusion 31 (1991) 2063. 


\subsection{EXPERIMENTAL STUDIES OF THE HEAT PINCH}

\subsubsection{INTRODUCTION}

Transport of energy and particles across magnetic flux surfaces in tokamaks has been known for many years to be anomalously large when compared with theoretical predictions based upon particle collisions. Models attempting to explain this transport normally assume that the time evolution of the temperature, $T$, of each species in the presence of some form of turbulence is still governed by a fluid-like equation of the form:

$$
\frac{3}{2} \frac{\mathrm{dnT}}{\mathrm{dt}}+\nabla \cdot \overrightarrow{\mathrm{q}}=\mathrm{Q},
$$

where $\overrightarrow{\mathrm{q}}$ is the heat flux. The heat sources and sinks are combined into $\mathrm{Q}$. In the diffusive model, the heat flux $\overline{\mathrm{q}}$ is assumed to be proportional to the gradient in $\mathrm{T}(\nabla \mathrm{T})$ and the proportionality constant is the thermal conductivity $(\kappa)$. The thermal diffusivity $(\chi)$ is related to $\kappa$ by $\kappa=n \chi$ where $n$ is the species density. The diffusivity could be a function of $\mathrm{T}$ and $\nabla \mathrm{T}$, which makes the equation nonlinear; however, net inward transport of energy cannot be explained by purely diffusive models even with $\chi$ a function of $T$ or $\nabla T$.

\subsubsection{OFF-AXIS ECH EXPERIMENTS}

The DIII-D $60 \mathrm{GHz}$ ECH system continued to be a key tool in performing critical experiments required for understanding and advancing the tokamak concept of magnetic confinement. ECH is a unique heating technology in that the energy is coupled to the electrons in a localized spatial region of the plasma. The location is controlled by adjusting the toroidal magnetic field thereby moving the resonance to the desired location. A series of experiments utilizing this capability have clearly demonstrated an anomalous inward flow of the added electron thermal energy, in contrast to the predictions of standard techniques used to model energy transport in the tokamak.

Previous experiments on DIII-D utilizing localized heat deposition with ECH have shown that the heat transport equation coupled with the above diffusion model cannot explain the observations. A term must be added which causes an inward energy transport for the electrons, up the temperature gradient. In these studies, the DIII-D ECH system was configured to launch microwaves from the high magnetic field side of the plasma at $60 \mathrm{GHz}$. Recent experiments in FY92 have further expanded the size of the heat pinch and narrowed the error bars. The 
experimental electron temperature profile shown in Fig. 4.7-1 provides immediate evidence of transport not in accord with purely diffusive models. The temperature profile should be flat inside of the heating location $\left(\mathrm{Q}_{\mathrm{ECH}}\right)$ if the off-axis heating is the only power input. In this case, calculations indicate that $90 \%$ of the input power is deposited outside of $\rho=0.3$ ( $\rho$ is the radial magnetic coordinate), yet the electron temperature profile remains peaked.

A power balance analysis is performed with the ONETWO tokamak transport code using measured plasma density and temperature profiles, the radiated power profile, and the experimental magnetic surface topology. This analysis shows that in order to maintain the measured electron temperature profile shown in Fig. 4.7-1, heat must be transported inward as shown by the negative electron heat flux $\left(q_{e}\right)$ (Fig. 4.7-2). The single-fluid heat flux is positive for all radii, however, and is greater than the neoclassical level. Experiments were done in FY92 to substantially increase the size of the inward electron heat flux by increasing the plasma density and decreasing the central ohmic power by lowering the plasma current. Extensive verification and error analysis of the $\mathrm{ECH}$ deposition profile, electron and ion temperature, $Z_{\text {eff }}$, and current density profiles was done.

Fig. 4.7-1. Electron and ion temperature profiles during offaxis ECH. Also shown are the ECH and ohmic power densities.

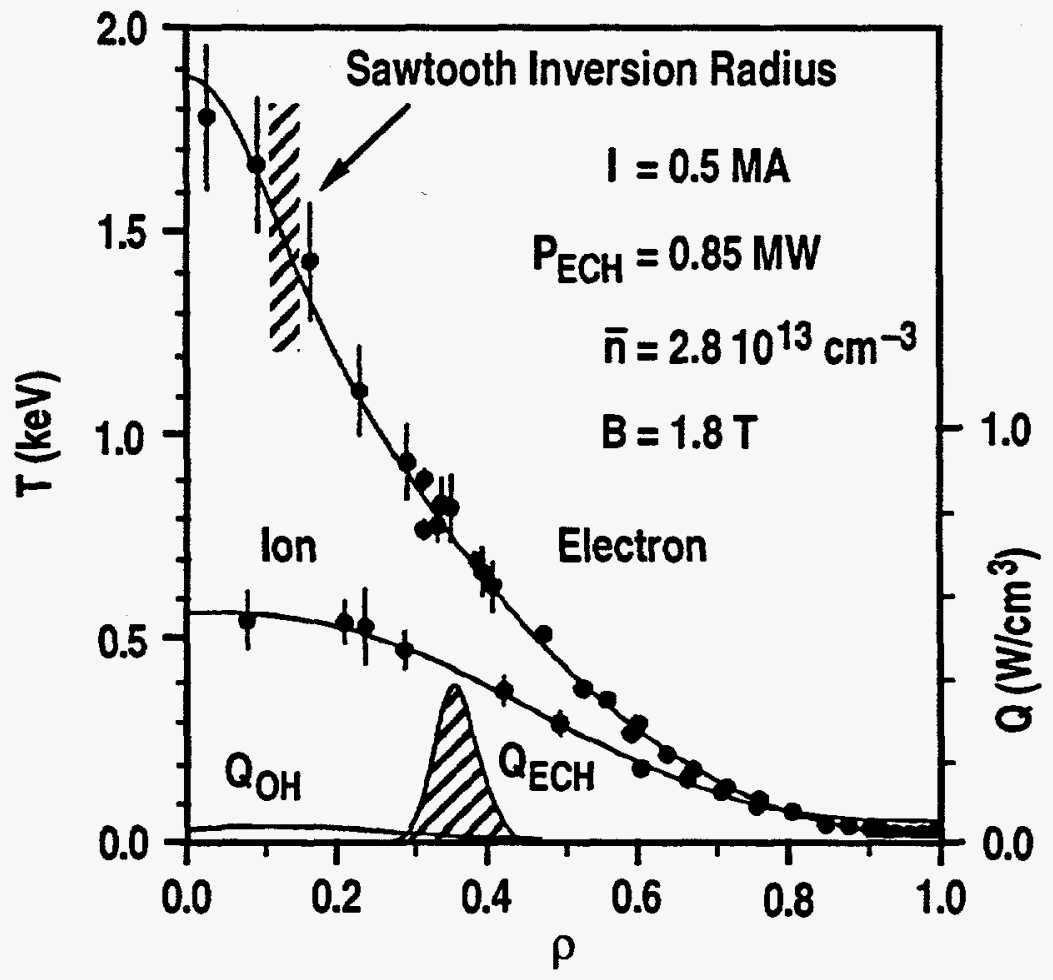


Fig. 4.7-2. Electron and ion thermal heat flux during off-axis ECH. This analysis is for the same data shown in Fig. 4.2-1.

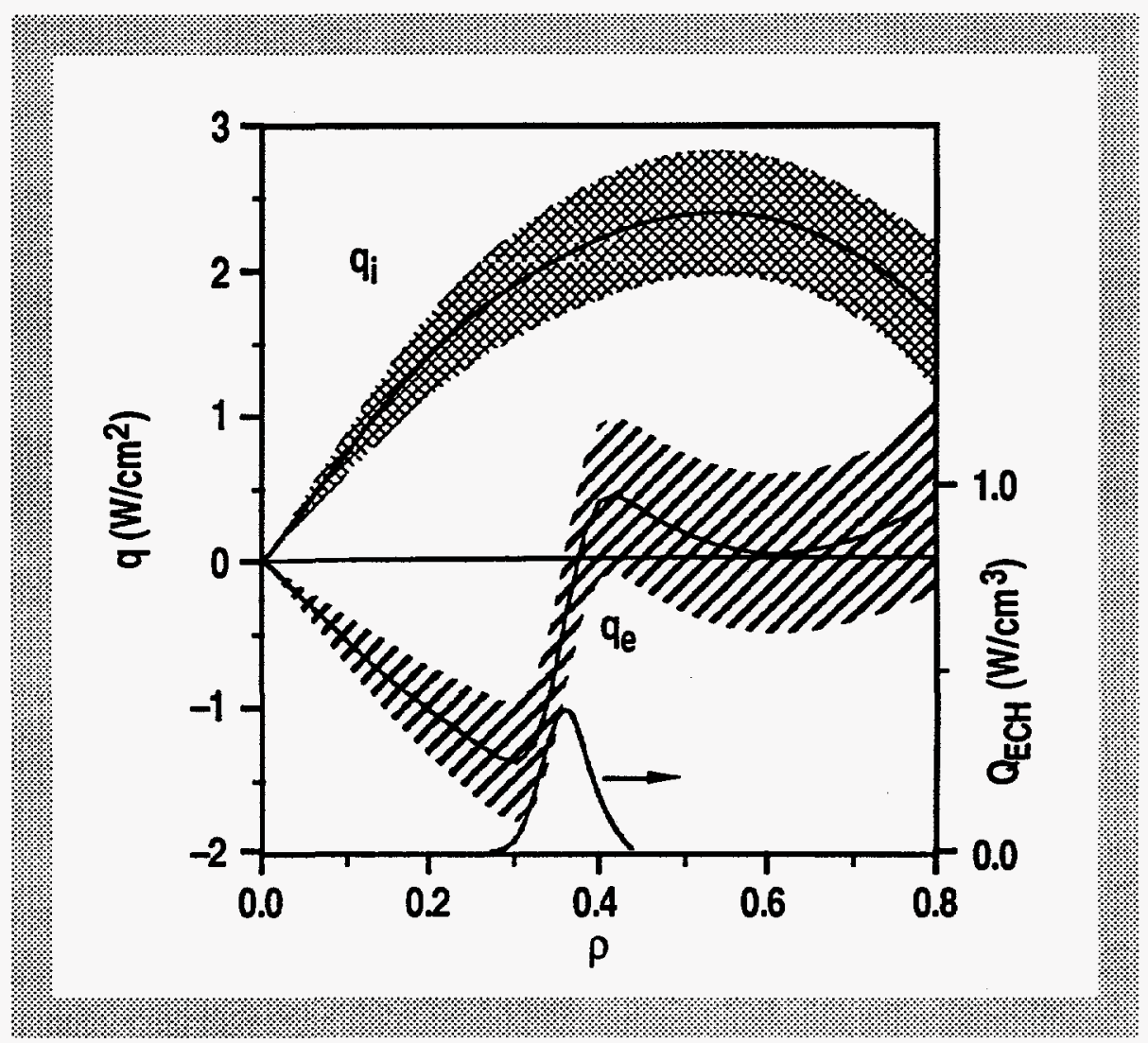

The observation of this electron heat pinch has stimulated theoretical interest. Several possible theoretical explanations are currently being evaluated. Experimental data on the heat pinch dependence on the plasma density, current, and shear have been obtained in FY92 and are being analyzed. This data should help identify the physical mechanism for the electron heat pinch.

\subsubsection{OFF-AXIS NBI HEATING EXPERIMENTS}

Experiments which focused on studying local plasma transport properties as a function of the heating profile were continued this past year. These off-axis neutral beam heating experiments, which are possible due to the flexible shaping capability of DII-D, were made routine with the introduction of the digital plasma control system. The H-mode experiments were conducted in a diverted deuterium plasma with values of plasma current Ip $=0.65 \mathrm{MA}$, toroidal magnetic field $B_{t}=2 \mathrm{~T}$, electron density $\left\langle\mathrm{n}_{\mathrm{e}}\right\rangle=4.5 \times 10^{19} \mathrm{~m}^{-3}$, aspect ratio $\mathrm{R} / \mathrm{a}=1.83 / 0.48=3.8$, and elongation $\mathrm{K}=1.5$. Off-axis heating was accomplished by vertically displacing the plasma $0.30 \mathrm{~m} ; 85 \%$ of the auxiliary heat was deposited outside the normalized 
minor radius of $\rho=0.4$. Application of just $2 \mathrm{MW}$ (one neutral beam source) of deuterium NBI at $75 \mathrm{keV}$ caused an $\mathrm{H}$-mode transition for all vertical positions.

This year's experiments concentrated on examining plasma transport on the long (10s of confinement times) time scale and on the short (fast ion slowing down time) time scale. The neutral beam systems were operated at $4 \mathrm{MW}$, a slightly reduced power level compared to last year's 7.5 MW, for the purpose of ensured plasma survival during several seconds of NBI. However, this operation had the added benefit of creating an electron temperature that was larger than the ion temperature so that two species of energy transport analysis could be performed. Analysis of the long time scale data was performed after $2 \mathrm{sec}$ of $\mathrm{NBI}$; the thermal energy confinement time was approximately $0.05 \mathrm{sec}$. At this time, the plasma density, temperature, and effective charge profiles had been in steady state for over $1.5 \mathrm{sec}$. Furthermore, analysis of the current profile using data from the motional Stark effect (MSE) diagnostic indicates that the current profile had also reached steady state at that time. The result of the analysis is shown in Fig. 4.7-3 which plots the ion and electron power balance diffusivity as a function of $\rho$. The power balance diffusivity was calculated from the 1-1/2 dimension ONETWO transport code assuming purely diffusive heat transport. Both the ion and electron diffusivity decrease dramatically inside the maximum heating zone which was at $\rho=0.5$. The ion diffusivity was reduced down to the neoclassical level which has been observed previously during hot ion $\mathrm{H}$-modes created with central NBI. However, the reduction of the electron diffusivity down to near the electron neoclassical level is new and represents a dramatically reduced level of electron transport.

Figure 4.7-4 shows the plasma profile evolution during the early phases of NBI. These two graphs depict $T_{e}$ as a function of $\rho$ at different times during the beam heating phase; the times are relative to the initiation of NBI. The top graph is a simulation of off-axis heating using the transport code ONETWO but assuming that the ion and electron diffusivities have values that are typically observed during central heating. As is expected assuming classical diffusion, inside of the heating source the electron temperature evolves to a slightly hollow profile with the peak value at the peak heating location. The bottom graph shows the measured value of $T_{e}$ by the Thomson scattering diagnostic. Clearly $T_{e}$ did not evolve as the simulation would predict. Instead, the measured electron temperature increased with time uniformly across the profile. The last profile, at $+56 \mathrm{msec}$, was just after the $\mathrm{H}$-mode transition and exhibits the usual steepening of the gradient near the plasma edge.

An alternate interpretation of the measure plasma profiles is to allow for an inward heat flow to exist during off-axis heating, thereby allowing the ion and electron diffusivities to remain 


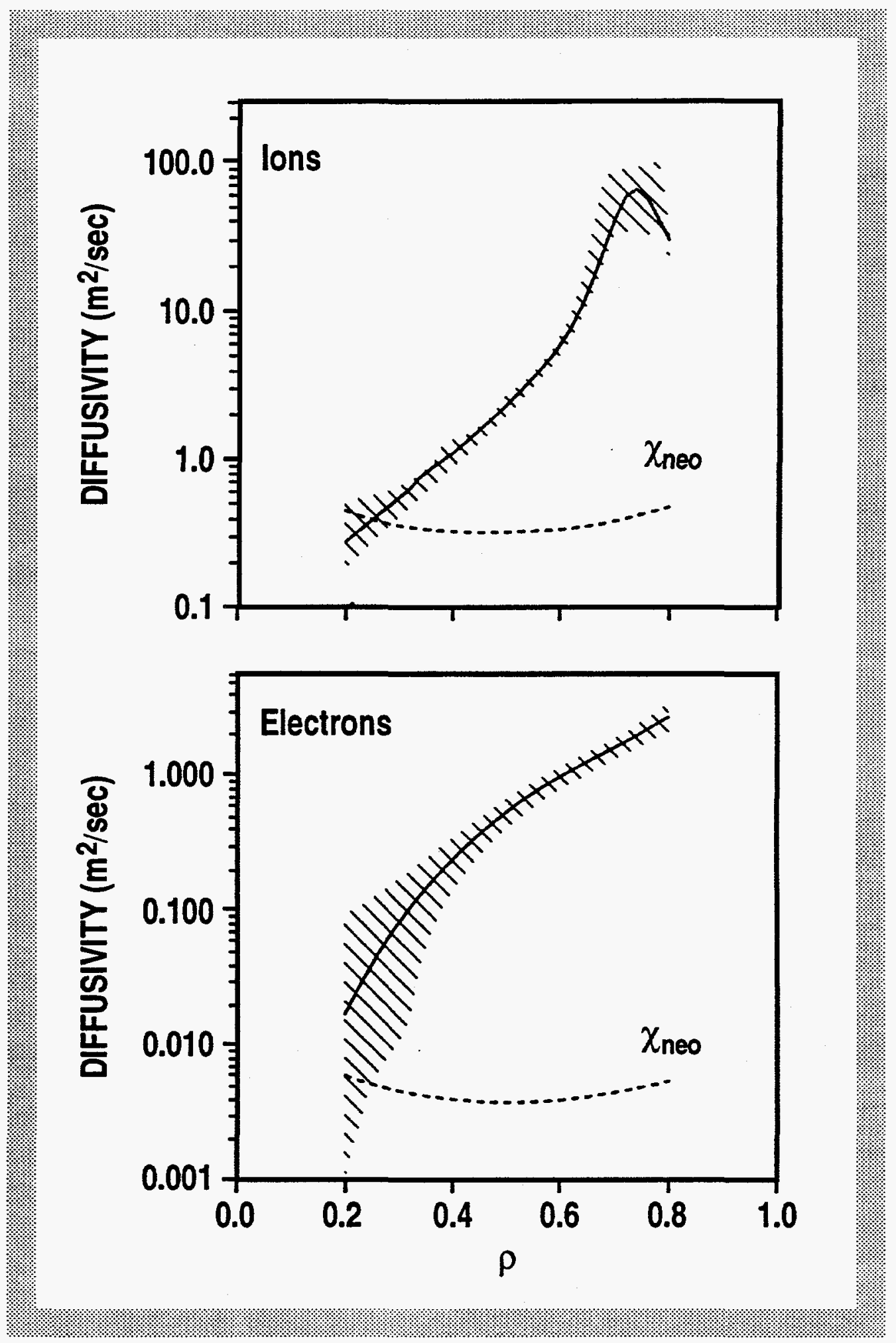

Fig. 4.7-3. Ion and electron thermal heat diffusivities for the case of off-axis NBI heating. 


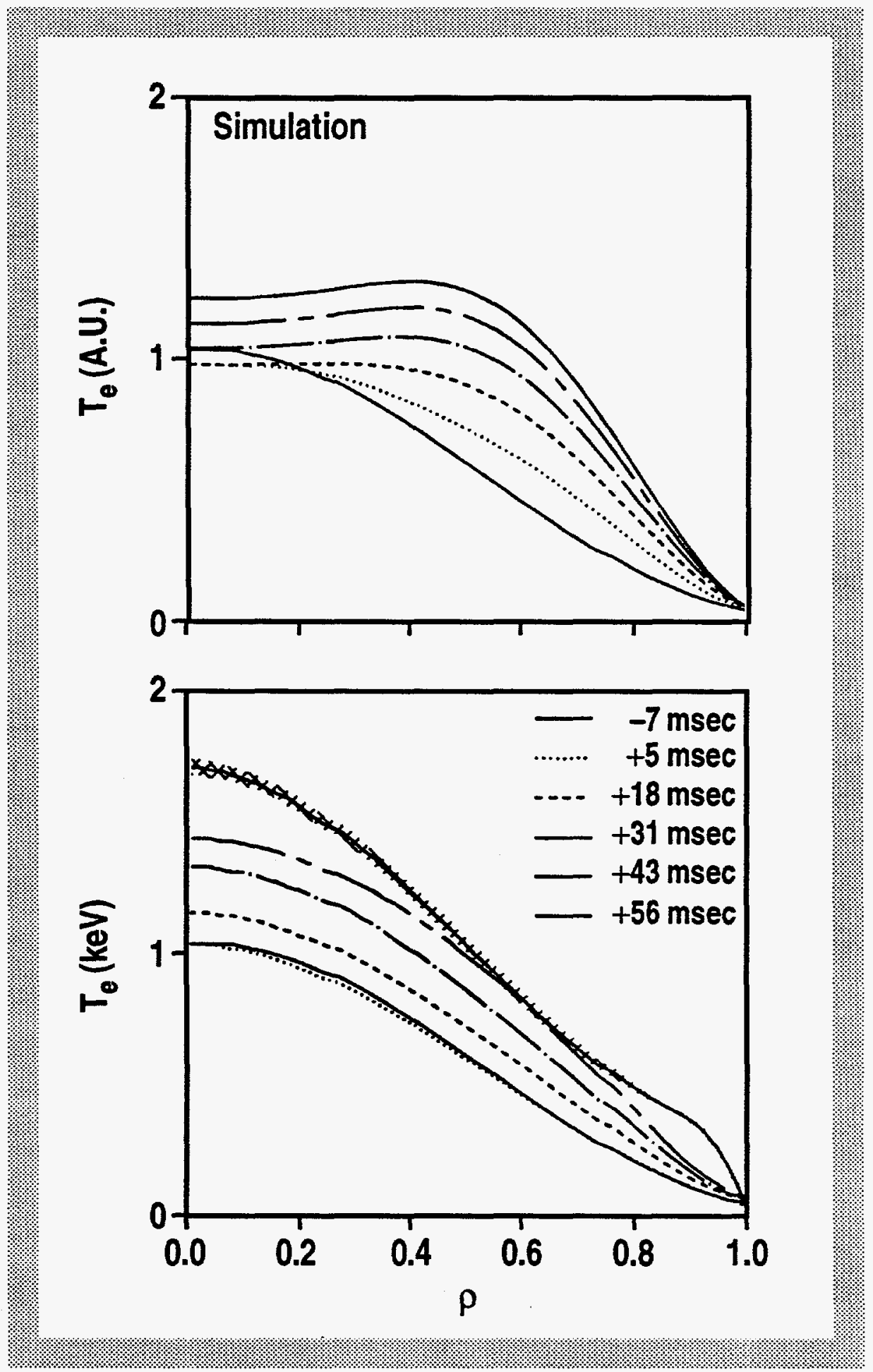

Fig. 4.7-4. Rapid time evolution of the electron temperature during off-axis NBI heating (bottom). Also shown is a simulation of the $T_{\text {e }}$ time evolution assuming diffusive transport with a $\chi_{e}$ determined from an axis heating. 
unchanged. The maximum power flow would be obtained at the peak of the auxiliary heating deposition, which would be similar to what has been observed during discharges with off-axis ECH. It is also possible that the appropriate local variables that describe local transport have not been identified or that local transport is not completely determined by local variables.

Results from DIII-D now demonstrate that the power balance diffusivity changes with the heating location for each of the two different heating schemes: $\mathrm{ECH}$ and NBI. Therefore, we are left to conclude that this plasma transport behavior is a general result and not specific to just one type of heating scenario. Furthermore, in the neutral beam case where heat is applied to both the ions and electrons, both species exhibit a reduced diffusivity inside of the heating zone. This type of transport behavior has been demonstrated during steady-state operation as well as during the highly transient time just after the initiation of NBI. In the future, we propose to operate discharges with a locally negative radial ion heat flux by attempting to create a hot ion $\mathrm{H}$-mode with off-axis heating. The low-target densities required for this experiment were demonstrated this past year and the addition of graphite tiling on the outer wall should help reduce impurity sources associated with large input power.

\subsection{FAST WAVE ELECTRON HEATING WITHOUT THE FARADAY SHIELD}

There are several potential advantages to operating an ion cyclotron radio frequency (ICRF) antenna without a Faraday shield: (1) significant simplification of the antenna design; (2) more reliable for long-pulse operation; (3) removal of a principal source of radio frequency (rf) sheaths, sputtering, and resulting impurities; and (4). significant reduction of the antenna losses. In general, a Faraday shieldless antenna is a much more reactor-relevant structure. However, careful comparison of shielded with unshielded antenna operation in a divertor tokamak is required to validate these advantages.

In a set of experiments performed during 1992, the Faraday shield was removed to compare antenna performance with and without a Faraday shield. The most apparent difference in the antenna performance was the observation of power-dependent antenna loading, which appeared to saturate at around $100 \mathrm{~kW}$. This type of nonlinear loading was not observed with a shielded antenna. To maintain an impedance match with a fixed tuning network during the pulse, a feedback scheme using the DIII-D digital control system was implemented, wherein the antenna plasma distance was feedback controlled so that the antenna loading was constant. The power handling of the unshielded antenna appeared to be lower than in the shielded 
configuration, though extensive conditioning procedures were not performed. No rf-specific impurity influx was observed at power levels up to $0.8 \mathrm{MW}$, except during antenna arcing.

Efficient direct electron heating by the unshielded antenna was obtained at moderate power levels. A comparison of FW heating using the unshielded antenna with $60 \mathrm{GHz} \mathrm{ECH}$ at $2 \mathrm{~T}$, shown in Fig. 4.8-1, shows that efficient heating does not necessarily require a Faraday shield.

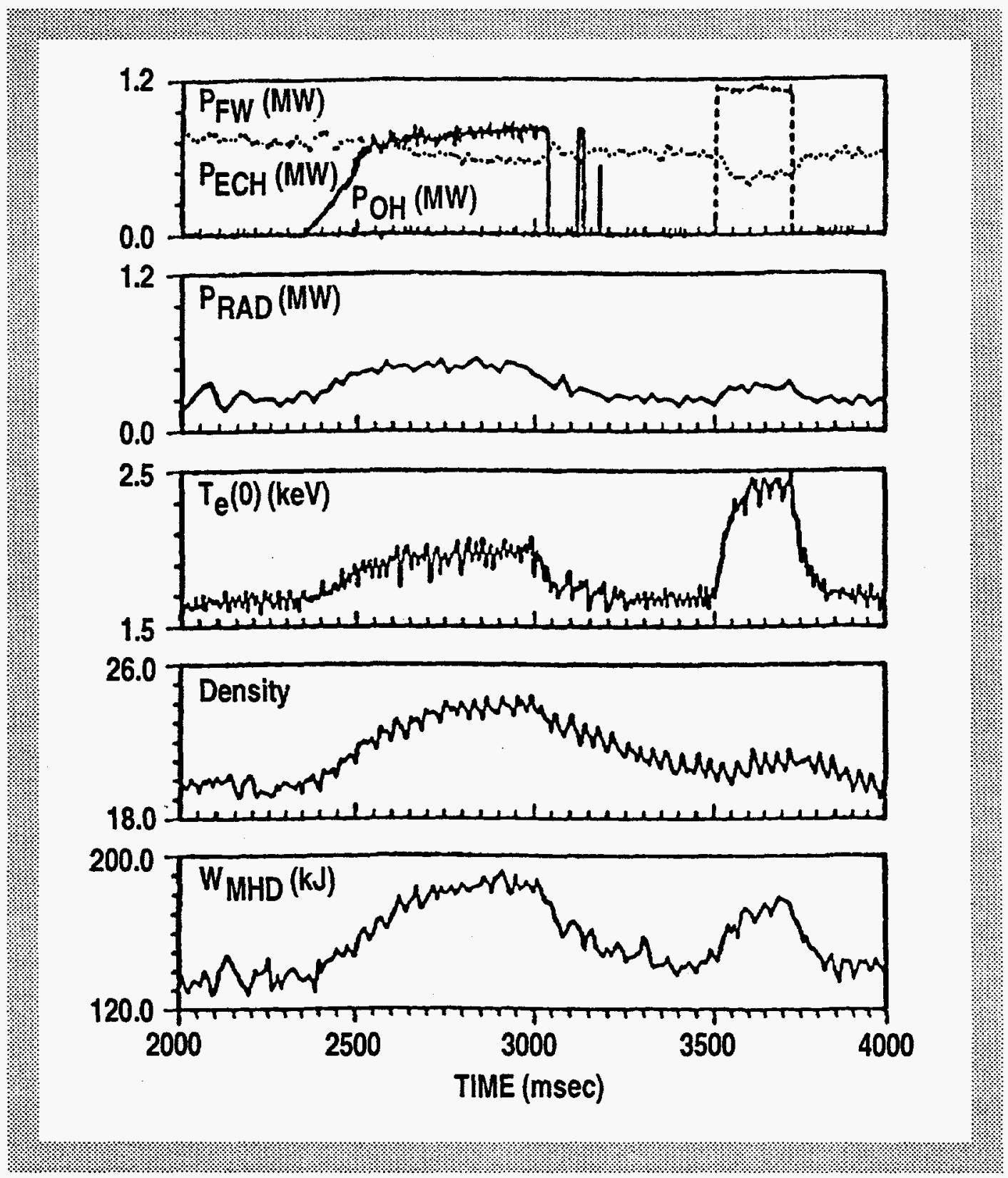

Fig. 4.8-1. Comparison of unshielded fast wave heating (solid line) and ECH (dashed line) for $B_{T}=2 T$. 
GA-A21186; FY92 DII-D Research Operations Annual Report

The antenna phasing was $(0, \pi, 0, \pi)$ for this shot. Compared to the ECH pulse, the FW pulse resulted in a higher density plasma at a lower temperature. The total stored energy for the two forms of rf heating were about the same. Lack of sufficient experimental time prevented a definitive conclusion to be reached concerning shielded versus unshielded operation. fast wave current drive (FWCD) experiments in the FY93 campaign will be performed with a new singlelayer Faraday shield mounted on the antenna. 

SECTION 5

OPERATIONS 



\section{OPERATIONS}

\subsection{TOKAMAK OPERATIONS}

\subsubsection{OPERATIONS SUMMARY}

During FY92, there were two major vents of the vessel for modification of existing equipment and installation of new equipment (Fig. 5.1-1). Between these vents, there was a relatively long period of alternating plasma operation and maintenance periods with only two short vents for minor in-vessel repairs. The total operating time for the year was approximately 14 weeks, during which time the machine availability was $77.6 \%$. A breakdown of the availability and the number of shots fired each month is shown in Fig. 5.1-2. Because of the frequent boronization of the vessel which began in FY91, the recovery from these short vents is rapid and the impact on the operating schedule is minimal. Despite a five-day vent in February to repair a damaged bellows in the fast wave current drive (FWCD) antenna, the start of plasma operations was only delayed by one day and good discharges were obtained much more rapidly than usual after a vent involving personnel entry.

\subsubsection{IN-VESSEL WORK}

After a brief two-week run period at the beginning of the year, the vessel was vented for approximately two months for the primary purpose of installing new and upgraded insulation for the advanced divertor ring and to install additional graphite armor on the outer wall. The fragile, glass-like Nextel fiber insulation that was originally used was replaced with a machinable ceramic material that is installed and fixed in precise locations. The ring successfully hi-potted to $5 \mathrm{kV}$ without arcing. In addition, some components were tested to $8 \mathrm{kV}$ before they arced, which is a significant improvement over the $3 \mathrm{kV}$ standoff capability of the earlier insulation design. A number of additional modifications were made to the surfaces of the plasma-facing insulators in order to prevent coatings deposited during plasma operation from compromising the insulation. During the subsequent quarter, the ring was used successfully for a number of experiments including 


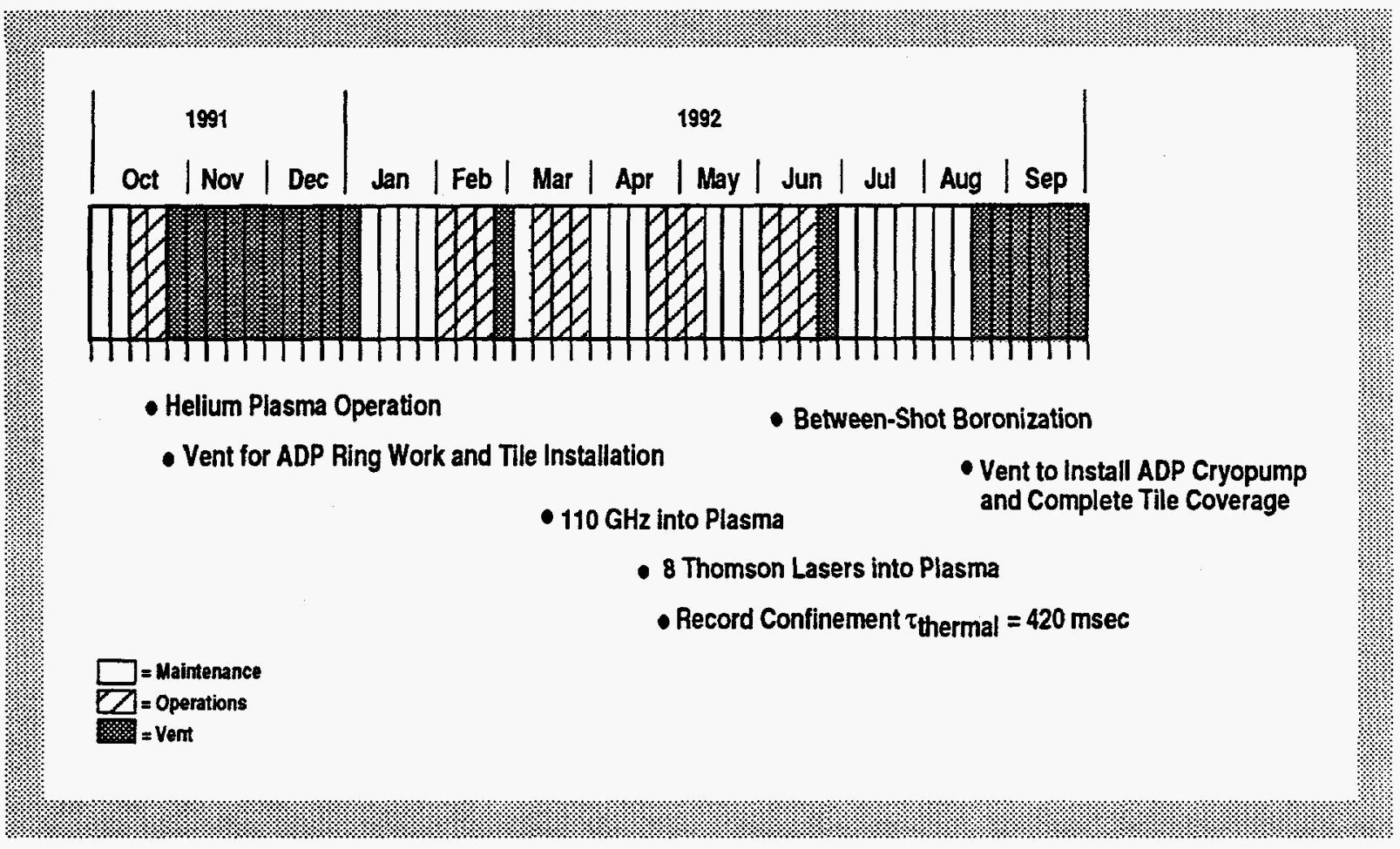

Fig. 5.1-1. DIII-D FY92 weekly operations schedule.

documentation of the scrape-off layer (SOL) characteristics and a novel scheme for inductionless startup using the ring bias. The second major task during this vent was the installation of additional graphite armor in the upper divertor region and in three separate poloidal bands on the outer wall. The purpose of this additional armor was to prevent melting of Inconel armor tiles that were observed in a number of specific locations on the outer wall. Subsequent inspection after the FY92 operating period indicated that the addition of the graphite tiles had successfully prevented further melting.

A second major vent began in August and will continue until the beginning of 1993 . The major tasks include the removal of the metal and bromine deposits from the surface of the existing graphite tiles, addition of new graphite tiles to provide nearly complete coverage of the Inconel wall, and installation of the divertor cryopump. A significant number of new or upgraded diagnostic systems are also being installed during this vent. A list of the major vent-related tasks is listed in Table 5.1-1. In order to remove the metal and bromine deposits from the tiles, all of the graphite tiles were removed from the vessel and have been grit blasted with a boron carbide grit which removes approximately 50 microns from the tile surface. The successful removal of the surface metal contamination was 


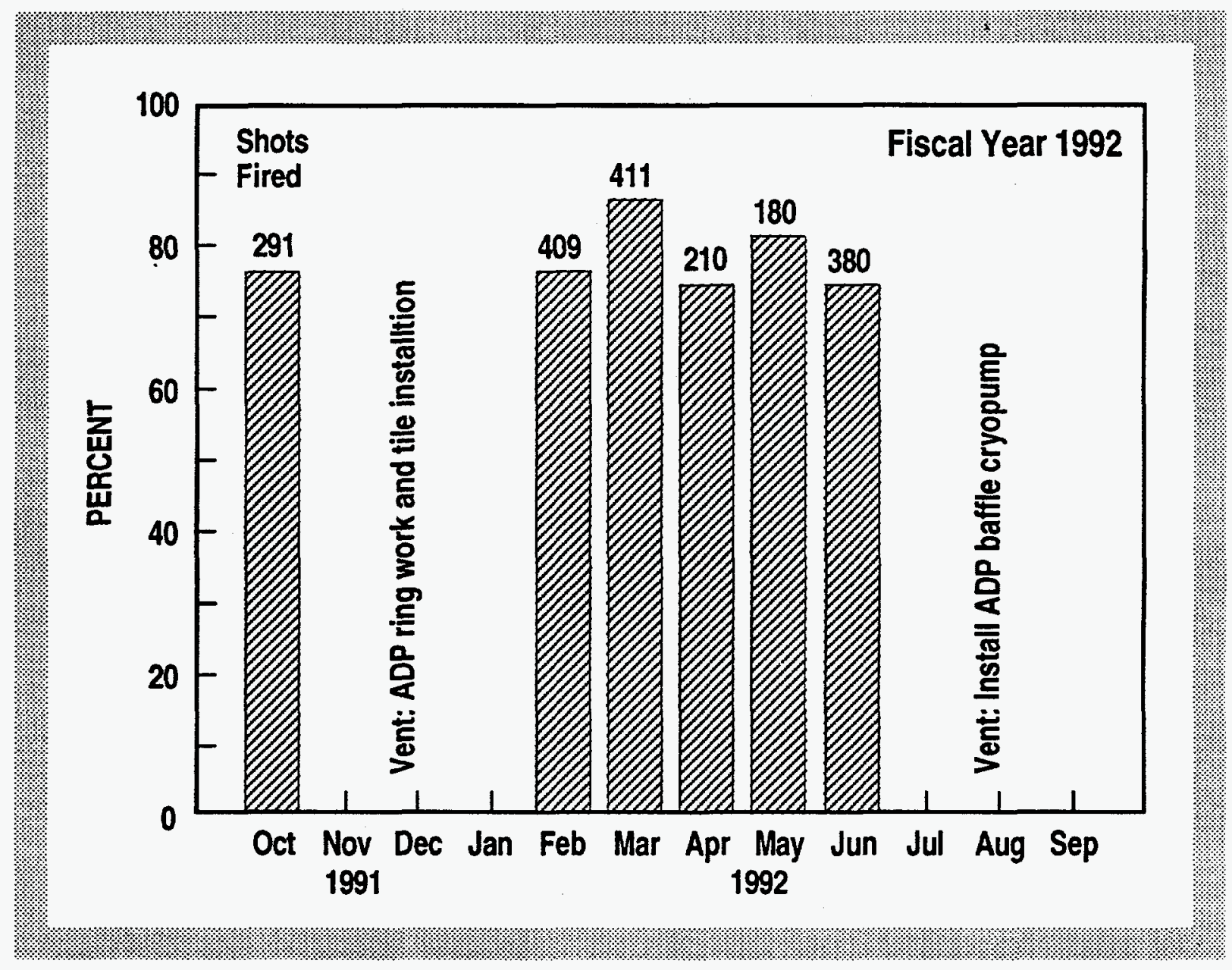

Fig. 5.1-2. DIII-D machine availability.

verified by a beta back-scattering system that was loaned to us by Sandia National Laboratory, Livermore (SNLL).

After grit blasting of the tile surface, the tiles were cleaned in an ultrasonic cleaner using alcohol and then subsequently outgassed to $1000^{\circ} \mathrm{C}$. Extreme care was taken in the specification of the outgassing procedure, qualification of the ovens used for baking, and testing of the finished tiles in order to minimize the recovery time between the end of the vent and the start of experimental operations. The tritium contamination of the tiles complicated the outgassing process and required significant testing before the baking to quantify the total amount of tritium expected, monitoring during the baking to detect and verify that we were below all regulatory limits for tritium release, and testing after the baking to verify that no equipment or personnel were contaminated by tritium above regulatory limits. 


\section{TABLE 5.1-1}

MAJOR VENT TASKS

\section{VESSELAND FiRST WALL}

Tile cleaning

New graphite tiles

Boronization gas line array

Vessel Illumination

\section{In-Yessel Diverton Harowabe}

Helium cryopump

Tile current array

ORNL He pressure gauges and baratrons

ORNL fast pressure gauges

Dlvertor Material Exposure System (DIMES)

\section{IN-VESSEL RF HARDWARE}

Faraday shield and antenna installation

Reflectometer

$180^{\circ}$ limiter removal

RF prove array

\section{Diagmostics}

Bolometer amay

Foil bolometer array

High-frequency magnetic probe array

Upper divertor $\mathrm{H}_{\alpha}$ monitors
Removal of metal and bromine surface layer

Provide nearly complete graphite wall coverage

Provide multiple gas lines to improve toroidal symmetry of boronization process

Expanded system to fully illuminate vessel interior for inspection of vessel under vacuum

Provide particle pumping in the DIII-D divertor

Expanded system to examine toroidal symmetry of SOL currents

Improve diagnostics for He removal studies

Relocate gauges to examine toroidal symmetry under baffle

Provide in-situ material testing capability

New ORNL Faraday shield for FWCD experiments

Measure density at front surface of FWCD antenna

Preparation for installation of additional if antenna

Measurement of the if field across from antenna

Higher resolution, multichord, dual viewport system - improved power balance calculations

Measurement of spatial distribution of radiation and anomalous particle loss

Measurement of high frequency fluctuations (e.g., TAE modes)

Relocate and increase number of views of upper divertor 
To eliminate local areas of high stress on the tiles due to surface irregularities in the vessel surface, copper foam gaskets were previously used to mount the tiles to the vessel. However, small flakes of this copper foam fell into the plasma discharges and became a major impurity source in DIII-D plasmas. An extensive qualification program was performed on a new, all-graphite gasket. This gasket has replaced the copper gasket. This should significantly improve plasma operations this coming year.

\subsubsection{VESSEL CONDITIONING}

There is probably no task more important for successful tokamak operations than conditioning the vessel surfaces. This became even more apparent during FY91 when a new higher confinement mode, $\mathrm{VH}$-mode, was discovered after the surface of the vessel was coated with a thin film of boron. The film reduced the metal contamination, reduced oxygen impurity contamination of the plasma, and reduced recycling of deuterium gas from the graphite tiles. During this past year, a number of modifications were made to the boronization operating system to improve its reliability, effectiveness, and ease of operation. These include additional cooling of the turbopump, operation of the turbo at a lower pumping speed to better match the throughput of the entire pumping system, remote control of the gas flow, a helium purge system for the secondary vacuum pumps, and the ability to operate the glow discharge in a pulsed mode. The modified operation of the turbo should both extend the life of the pump and also significantly improve the ease of operating the boronization system. The purge eliminates the need to measure the secondary leak rates prior to each boronization. The capability of pulsing the glow discharge permits the diborane gas to more uniformly distribute around the vessel before depositing on the vessel surface.

Analysis of the boron deposits on toroidally spaced tabs on the inner vessel surface was performed and indicated a decay length in the boron deposit on the wall of $2.2 \mathrm{~m}$ from the gas inlets. The original boronization gas system had only two inlet ports which resulted in the strong toroidal asymmetry in the depth of the boron layer. Based on this, an array of 16 gas lines were installed inside the vessel to provide a more uniform coating of the plasma-facing surface.

In order to more quantitatively evaluate vessel cleanliness and assess long-term trends in impurity behavior, a set of plasma reference discharges have been established for both single-null and double-null divertor discharges. These reference shots are now routinely taken as the first shot of every day (when compatible with the hardware). Computer programs 
were written to permit rapid reloading of all relevant machine control parameters for these shots and the effect on experimental time has been minimal. The data from these reference discharges will be used extensively in the post-vent period in FY93 to assess the cleaning process and to determine the effect of the addition of the graphite tiles on impurity content and recycling.

In order to minimize the conditioning time required following the installation of the new graphite tiles, extensive testing and optimization of the glow discharge cleaning system was conducted at the end of the operating period in FY92. Based on these tests, a number of modifications are being made to the system hardware and new methods will be utilized in the post-vent period. These changes include the use of the $0^{\circ}$ limiter as an electrode, moving the two existing electrodes closer to the plasma, operation of the glow discharge at higher current and voltage, the use of argon and neon in addition to helium, and the use of radio frequency (rf) assist to permit glow initiation and operation at lower pressure. We are hopeful that the use of these new techniques will result in an efficient recovery and operating period after plasma operation resumes in early 1993.

\subsection{NEUTRAL BEAM OPERATIONS}

\subsubsection{OPERATIONS SUMMARY}

Fourteen weeks of plasma heating experiments were supported by neutral beams in FY92. For all but the last two weeks of the operating year, seven systems were available, with the eighth power supply on loan to the electron cyclotron heating (ECH) system. Two weeks before the end of operations, the eighth system was converted back to neutral beam use, and the ion source was conditioned and injected for the last week of physics experiments. In June, operations ceased for an extended vent and maintenance period while new tokamak armor tile and the advanced divertor cryopump were installed. During this latter period, an aggressive program of ion source and beamline repair and maintenance was undertaken by the neutral beam group, in addition to our personnel supporting many of the tokamak upgrade tasks.

\subsubsection{SYSTEM IMPROVEMENT AND MAINTENANCE}

As foreshadowed by an increase in ion source downtime last year, this year for the first time ion source repairs overtook power supply problems as our leading cause of downtime. 
This was primarily due to two reasons: one being arcing between insulator gaskets in the arc chambers of several ion sources, a relatively minor problem repairable here at General Atomics (GA). The other problem has been of more concern: the failure of internal cooling passages of two separate ion sources. In one case, the $150^{\circ}$ Left ion source was pressurized with water, and the resultant forces distended the ion source mounts and adjustable bellows. The ion source broke loose and came to rest against the rear of the ion source enclosure. This leak was traced to the cooling passages in two molybdenum plates which mask the edges of the accelerator grids from the arc chamber. In the second case, leaks developed in five bellows associated with the $210^{\circ}$ Left ion source grid rails. This repair could not be attempted at $\mathrm{GA}$ and the ion source sent to Burle Industries, the successors to RCA, for repair. Careful investigation of these failures has determined them to have been caused most probably by water hammer effects. The closure of the ion source cooling water return paths can result in transient pressures in some cases approaching $2000 \mathrm{psi}$. To guard against this possibility in the future, we have undertaken a program of replacing all 72 ion source cooling return water valves with multiturn valves, making it virtually impossible to suddenly close the return flow.

Given the opportunity afforded by the tokamak vent period, all ion sources were scheduled for removal and lab inspection during the vent period commencing in July. No major problems were found with the remaining ion sources. One unit with two separate failed components was shipped to Burle, and is due back at GA in early March 1993. In the meantime, the one spare unit will be installed in its place. We will be giving high priority to finding the operational time to condition the repaired ion source upon its return without interfering with tokamak physics operations. This conditioning could take several weeks.

During this same vent period, all beamlines were inspected, along with the ducts between the beamlines and torus. The beamline internals were found to be in good shape, except for a minor water leak in the $30^{\circ}$ Left calorimeter cooling path, internal to the beamline. Inspection of the drift ducts showed that there was little sign of the melting that has previously been observed in these areas. This good news is attributed to our new realtime photodiode interlock, which interrupts the beam if the visible light emitted by runaway reionization in the drift duct is detected. Operational experience has shown now that this condition is rarely encountered during regular operation, but is a serious problem to be monitored closely during startup periods after dirty vents. The problem is also encountered 
when the tokamak toroidal field direction is changed, changing the areas in the duct which are impinged by reionized particles.

During the vent period, an inspection was made of the sealing surfaces of the $330^{\circ}$ and $150^{\circ}$ beamline-torus isolation valves (TIVs). The remains of a ball bearing cage was found in the trough of the $150^{\circ}$ valve and was determined to have come from the paddle guides of the valve itself. Visual inspection of the remaining bearings revealed galling and wear. Based on this evidence, the paddles of all four TIVs were removed from the valve bodies to the high-bay work area for inspection - a major operation. New bearings have been ordered from the Swiss manufacturer of the valves, and the valves will be back in service by the end of CY92.

Improvements to the Doppler Shifted Spectroscopy system have resulted in more reliable and consistent measurements of the ion species, which has provided us with more accurate beam power measurements.

A new ion source waterflow calorimetry system measures the power deposited on the accelerator grids for better power accountability. This grid power measurement also verifies that we are operating well within the designed grid power loading limit. Longer range plans call for using this system to provide a realtime beam interrupt capability to prevent damage during longer pulse lengths.

\subsubsection{SYSTEM AVAILABILITY}

Overall average availability for the year is very good at $91.5 \%$, which compares to 91.1\% for FY91. Availability of the neutral beam systems by month is shown in Fig. 5.2-1. Since often the emphasis is on providing beam for tokamak physics diagnostics, or providing limited comparable power levels to $\mathrm{ECH}$, there are times when only a small fraction of the available beam power is being used. This explains the difference between the "Available" and "Injecting" categories.

In November 1991, there was a tokamak vent for the installation of new advanced divertor ring power and cooling feedthroughs and to repair a damaged ECH waveguide. Neutral beams came up well after this vent with high availability. July marked the beginning of an extended vent period. The various causes for downtime are shown in Fig. 5.2-2. Vacuum, cryogenic, and computer problems each count for less than $1.5 \%$ of the total. 


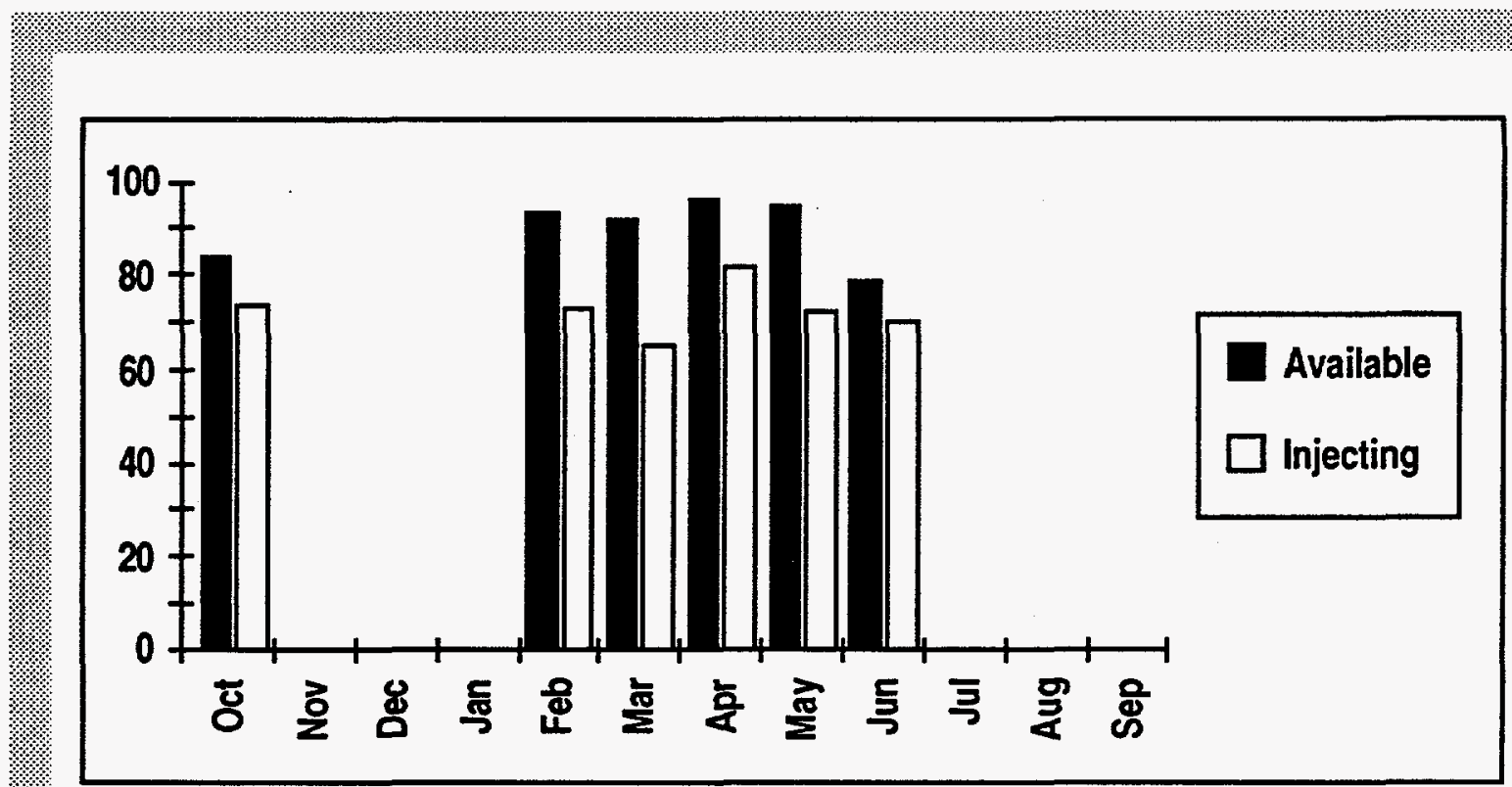

Fig. 5.2-1. Neutral beam availability by month.

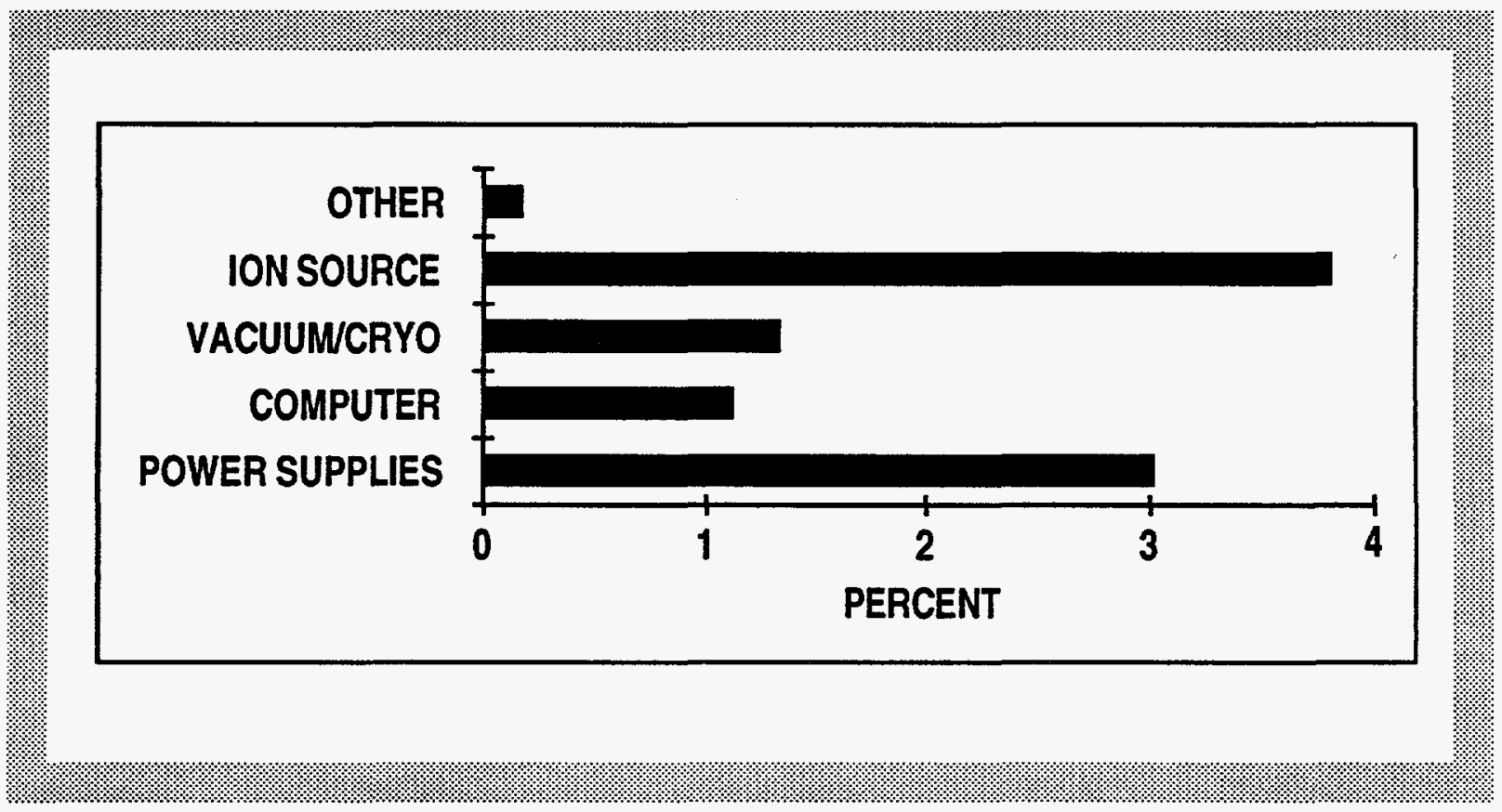

Fig. 5.2-2. Causes of downtime by category (percent of total operating time). 
In 1992, a power supply reliability task force was established and has made recommendations which are expected to improve power supply reliability further. The group is making a concerted effort to make hardware and operational changes to preserve the huge investment present in the common long-pulse ion sources. We have had some repair parts machined locally and are engaged in ongoing discussions with the manufacturer to try to provide for spares and service in the future.

\subsection{ELECTRON CYCLOTRON AND ION CYCLOTRON HEATING OPERATIONS}

\subsubsection{ELECTRON CYCLOTRON HEATING OPERATIONS}

During FY92, the $60 \mathrm{GHz}$ ECH system was used to support 13 days of plasma experiments. The use ranged from plasma startup to assisting the ion cyclotron heating (ICH) system in current drive experiments. Even though the year started out with eight operating gyrotrons, one of the gyrotrons developed a small water leak that made it inoperable. The cost of repairing this gyrotron was outside the available budget and, therefore, the repair of this unit was not pursued.

During the fall 1991 DIII-D vent, the waveguide sections between the wall and the section known as the "hockeystick" were discovered damaged. In four of the eight operational guides, holes melted through the walls. It was felt that this damage was caused by plasma streaming behind the tiles. New tiles have been installed which should eliminate this problem. When the DIII-D vessel was vented in June 1992, additional melting was observed in the throughport extension at $255^{\circ}$. This melting was not a repeat of the past problem but was attributed to the use of a thin wall bronze section, which overheated during use. These bronze pieces have been replaced with thicker wall copper sections.

The $110 \mathrm{GHz}$ system was successfully operated with a DIII-D plasma to demonstrate the total system performance. The shutdown of the machine to perform in-vessel modifications have put off any further $110 \mathrm{GHz}$ experiments until next year.

\subsubsection{FAST WAVE HEATING AND CURRENT DRIVE OPERATIONS}

Transmitter and transmission line problems throughout the year severely limited the amount of operating time of the ion cyclotron radio frequency (ICRF) system. Nine 
days of heating experiments and one day of current drive were achieved this year. The major events were an open circuit in one of the antenna feeds caused by the braze on an inner conductor bellows failing due to excessive rf heating. The phase shifter in Line A failed to respond to adjustment commands. Upon inspection, it was found that the insulating rod connecting the drive to the phase shifting stub had been cut in half by rf arcing. Inspection of Line B showed similar arc damage but the rod had not yet separated. It was determined that the $G 7$ material used for the insulating rod was overheating. These rods were replaced with Delrin rods which have a lower rf loss factor. An arc-detecting system has been added to all phase shifters and stub tuners to protect the system from arc damage in the future.

With the help of ORNL rf engineers, the Final Power Amplifier (FPA) was modified to take advantage of the full $2.25 \mathrm{MW}$ that the new X-2274 Tetrode would provide. Tuning was performed to reach the $2+\mathrm{MW}$ level; however, $\mathrm{rf}$ breakdown in the system prevented the achievement of this goal. After inspection, damage was found in the driver harmonic trap where arcing was taking place across a Teflon capacitor. A new Teflon capacitor was fabricated with a longer creep path. An optical arc detector was added in this section for added protection.

\subsection{DISRUPTION STUDIES AND PLASMA CONTROL}

\subsubsection{INTRODUCTION}

Significant progress has been made in advancing the disruption studies program on DIII-D. The major emphasis has been on analysis and simulation of DIII-D disruption processes that can have an impact on the International Thermonuclear Experimental Reactor (ITER) design. To this end, the work has involved collaborative efforts with other laboratories on experiments and on data analysis, disruption simulation, and code development. The major efforts have included: (1) computer simulation and analytic modeling of plasma motion during disruptions; (2) the development of an operations and disruption database to provide statistical information and guidance for the next generation machines; (3) locked mode studies and extrapolation of results to ITER for ohmic, rf, and beam-heated discharges; and (4) analysis of density and temperature profiles during disruptions. 


\subsubsection{Disruption Modeling and Plasma Motion}

An understanding of the plasma trajectory during a major disruption is essential for the proper design of the divertor and vacuum vessel in large, high current tokamaks such as ITER. Through operating space restriction or active control, such an understanding may allow the large magnetic forces and material erosion associated with the disruption to be confined to vessel and divertor plate regions which have been designed with appropriate resiliency. Disrupting single-null diverted discharges in DIII-D generally move toward the X-point during the current quench, coming in contact with the divertor plate until the plasma current is entirely lost. However, some disrupting single-null discharges move away from the $\mathrm{X}$-point, and double-null cases are often seen to move in a predominantly radial direction following disruption. In order to understand the origin and nature of these different motions, we have been developing a fast plasma simulation code and an analytic model of the vertical motion of disruptive single-null plasmas.

During FY92, there has been a collaborative effort with the TRINITI laboratory in Russia on the continued development and benchmarking of the DINA 1-1/2 D resistive magnetohydrodynamic (MHD) and transport simulation code. The code has been configured to simulate steady-state discharges and disruptions in DIII-D. Extensive development and benchmarking has been done to validate the resulting DINA-DIII code version against DIII-D experimental data. Performance of shape control algorithms, equilibrium calculations, and evolution of shape parameters during shot simulations have been shown to produce good agreement with experimental data. During this process, the user interface and the efficiency of various algorithms have been significantly improved. A several millisecond disruption simulation or a several second-shot simulation can be calculated in minutes of CPU time on a VAX or workstation.

A new version of the DINA plasma simulation code with a basic halo current model, a new current profile mixing model, and subroutines with improved convergence characteristics is now running at GA. Using the new model, simulations were performed for cases with various changes in internal inductance during the disruption and for cases with and without active feedback control of the plasma position. The simulations confirmed previous results with a simple current mixing model: sufficiently large changes in the internal inductance cause motion toward the $\mathrm{X}$-point, and active control does not significantly affect the plasma motion for disruptions with a current decay of several milliseconds. 
Work is continuing on the development of an enhanced halo current model in DINA which is fully self-consistent. The basic implementation of this enhanced model has now been completed, including the full algorithm and a detailed poloidal current path. Testing of this new version is now in progress. Work has also begun on applying the validated DINA-DIII code development to a new version, DINA-ITER, which will include ITER geometry, a front end for accepting proposed ITER equilibria, and a back end for interfacing with structural stress analysis codes. The goal of this work is to develop a fast, user-friendly simulation code which can be used for the ITER design effort. To provide faster response time in the DINA calculations, the DINA code is being converted from the VAX operating system to the UNIX operation system for use on an HP730 workstation.

To better understand the plasma motion, an analytic model of the vertical motion of disruptive single-null diverted plasmas has been developed. The model indicates that plasma disruptions during the current flattop will tend to ultimately move away from the $\mathrm{X}$-point if following the thermal quench both the change in internal inductance is sufficiently low and the plasma current centroid is sufficiently far from the midplane and located on the opposite side of the midplane from the $\mathrm{X}$-point. Calculation of forces acting on the plasma during a disruption for the DINA code are consistent with the model.

In a related effort, benchmarking of the Tokamak Simulation Code with a plasma halo current model has been completed by calibration against a DIII-D vertical displacement episode (VDE). The VDE consists of vertical drift, thermal quench, and current quench. A complete dataset for the DIII-D discharge was provided to R. Sayer of Oak Ridge National Laboratory (ORNL) who performed the simulation. Inclusion of a $1-4 \mathrm{eV}$ halo surrounding the main plasma was found to be necessary to match simulation and experimental results for the plasma current decay rate, trajectory, toroidal and poloidal vessel currents, and magnetic probe and flux loop values for the entire VDE. Simulation of more recent DIII-D discharges with measurements of the electron temperature in the halo region are now beginning.

\subsubsection{Disruption Database}

In order to provide statistics which can be used to characterize disruptions, their type and frequency, and help identify disruption-free operating regimes, a database of operational parameters and disruption data has been created. Data can be analyzed directly from the database or displayed graphically. Presently, 4000 shots have been analyzed from 
the existing DIII-D database and both old and new discharges continue to be added. The database contains a large variety of disruption-specific parameters including peak and average current decay rates, loop voltage, peak density during the disruption, vessel motion during disruptions, safety factor, plasma shape, and all equilibrium parameters immediately preceding the disruption and during the nondisruptive phase of the discharge and vertical stability parameters.

Using the database, various dependencies have been examined. The fraction of shots that disrupt on DIII-D is typically $10 \%-20 \%$ of all plasma shots; at currents above 2.5 MA the disruption rate rises to $50 \%$; the disruption rate increases at low $\mathrm{q}$; the peak vertical vessel displacement, the peak current decay rate, and the amplitude of the "bump" or rise in current following the thermal quench scales with the predisruption plasma current.

Preliminary analysis also indicates that a significant fraction of the plasmas do not disrupt at the peak stored energy of the discharge or at the maximum plasma current. Many of the disruptions occur either during the rampdown, after the auxiliary heating power has been removed or reduced, or after the thermal energy has decreased due to slow, nondisruptive loss mechanisms in the plasma. These factors will be included in the analysis to provide more meaningful statistics and guidance.

In order to understand the apparent lack of runaway electrons in the DIII-D disruptions, a survey of hard X-ray emission following disruptions was conducted on 102 shots. The disruption distribution was: $11 \%$ disrupted in current rampup, $64 \%$ disrupted in flattop, and $25 \%$ disrupted in rampdown. The survey failed to indicate the presence of any runaway electron production, in agreement with earlier statements concerning the lack of runaways in DIII-D disruptions. Although hard X-ray bursts were observed in many of the disruptions, the toroidal symmetry of these signals and their appearance significantly before the current quench indicates that they are not a signature of disruption-generated runaways. Rather, they are likely related to the loss of fast electrons at the onset of the thermal quench.

\subsubsection{Locked Modes}

Irregularities in the winding or alignment of poloidal or toroidal magnetic field coils in tokamaks make resonant low $\mathrm{m}, \mathrm{n}=1$ static error fields. Experiments performed on DIII-D and other tokamaks have clearly shown that otherwise stable discharges can become 
nonlinearly unstable resulting in locked modes and subsequent disruption when subjected to these modest $m=2, n=1$ external perturbations. Using both theory and results of a coordinated set of error field/locked mode experiments on DIII-D, JET, and COMPASS, the critical $m=2, n=1$ applied error field for the locked mode instability in ITER was calculated for discharges with ohmic, neutral beam, or $\mathrm{rf}$ heating. The results indicate that ITER should be considerably more susceptible to locked modes than smaller devices because of the slower rotation speed. Ohmic discharges are predicted to be most sensitive. Although there is no momentum input during rf heating, the critical error field requirements are less severe than with ohmic heating because the higher electron temperature during rf heating increases the diamagnetic drift velocity which should reduce the sensitivity to locked modes. Because of the high momentum input during neutral beam injection, sensitivity to error fields is lower than in either rf or ohmic discharges. However, there is a strong decrease in the allowable error field as the normalized beta increases because the current profile becomes more unstable to tearing modes as beta increases. Even co-injected neutral beam discharges (at a normalized beta of 3 ) in ITER will require keeping the relative 2,1 error field, $B_{21} / B_{T}$, well below $1 \times 10^{-4}$. Experiments are planned in the upcoming year to further investigate the role of error fields on discharge reliability at high beta and low safety factor and during the current rampdown phase of the discharge.

\subsubsection{Profile Measurements}

Data has been obtained and analyzed on the behavior of the density and temperature during disruptions using the multipulse Thomson scattering system and the fast time response of the $\mathrm{CO}_{2}$ interferometer. A rapid rise (appoximately $200 \mu \mathrm{sec}$ ) in density occurs toward the end of the thermal quench and the density peaks at the edge of the plasma with a hollow profile in the main body of the plasma. The peaking of the density on the edge will strongly inhibit the generation of runaway electrons and should provide valuable data to improve the modeling of the disruption runaways. In the discharges examined, the temperature in the main body of the plasma varies from 50 to $200 \mathrm{eV}$ and appears to correlate with the rapid density increase. The measured electron temperature is consistent with the rapid density increase being due to carbon influx from the wall. Measurements of the electron temperature in the SOL have also been obtained and are in the range of 10 to $20 \mathrm{eV}$. In FY93, additional experiments are planned to take systematic measurements during both the thermal and current quench phases of the disruptions. 


\subsubsection{PLASMA CONTROL}

An advanced plasma control system has been implemented for the DIII-D tokamak using digital technology [5.4-1,5.4-2]. This control system is designed to provide feedback control of all aspects of the tokamak discharge including the discharge position and shape, the total plasma current, the electron density, and the total stored energy. The system is expandable to allow future control of current density and plasma pressure radial profiles. Development of this system is expected to lead to control system technology appropriate for use on future tokamaks.

The advanced plasma control system was made operational on DIII-D during the spring of 1992 [5.4-3] using prototype hardware. It gained rapid acceptance due to ease of use and the ability to quickly make plasma shape changes. Examples of five different discharge shapes that were produced in the first few weeks of operation with the new system are shown in Fig. 5.4-1. Improvements were noted in the reliability and reproducibility of discharges. During this initial period of operation of the digital system, the shape control algorithms that had been in use on the previous analog control system were used. In the next stage of work, advanced shape control methods will be implemented that will provide increased precision in shape control through real-time adjustment of the control algorithm to changes in the shape and discharge parameters.

Two new feedback control capabilities were easily implemented with the new control system: (1) control of the total stored energy, and (2) control of the ICRF antenna load resistance [5.4-3]. Feedback control of the total stored energy was implemented through pulse width modulation of the injected neutral beam power. This is the first time that stored energy control of this type has been accomplished in a large tokamak. An example is shown in Fig. 5.4-2. In the discharge shown in Fig. 5.4-2, the feedback parameter was the plasma diamagnetism which is proportional to the total stored energy. By varying the total input power, as shown in this figure, the control system maintained the diamagnetism at a preprogrammed level as a function of time, even in the presence of several large bursts of edge localized instabilities (shown by the spikes in the photodiode signal) which cause a loss of stored energy. This capability for feedback control of stored energy was used to significantly improve the performance of VH-mode discharges. In ICRF heating experiments, the control system was used to maintain the load resistance of the rf antenna at a constant, preprogrammed level. During the discharge, the control system continuously calculated 


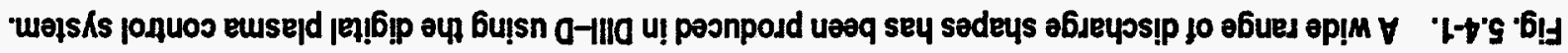

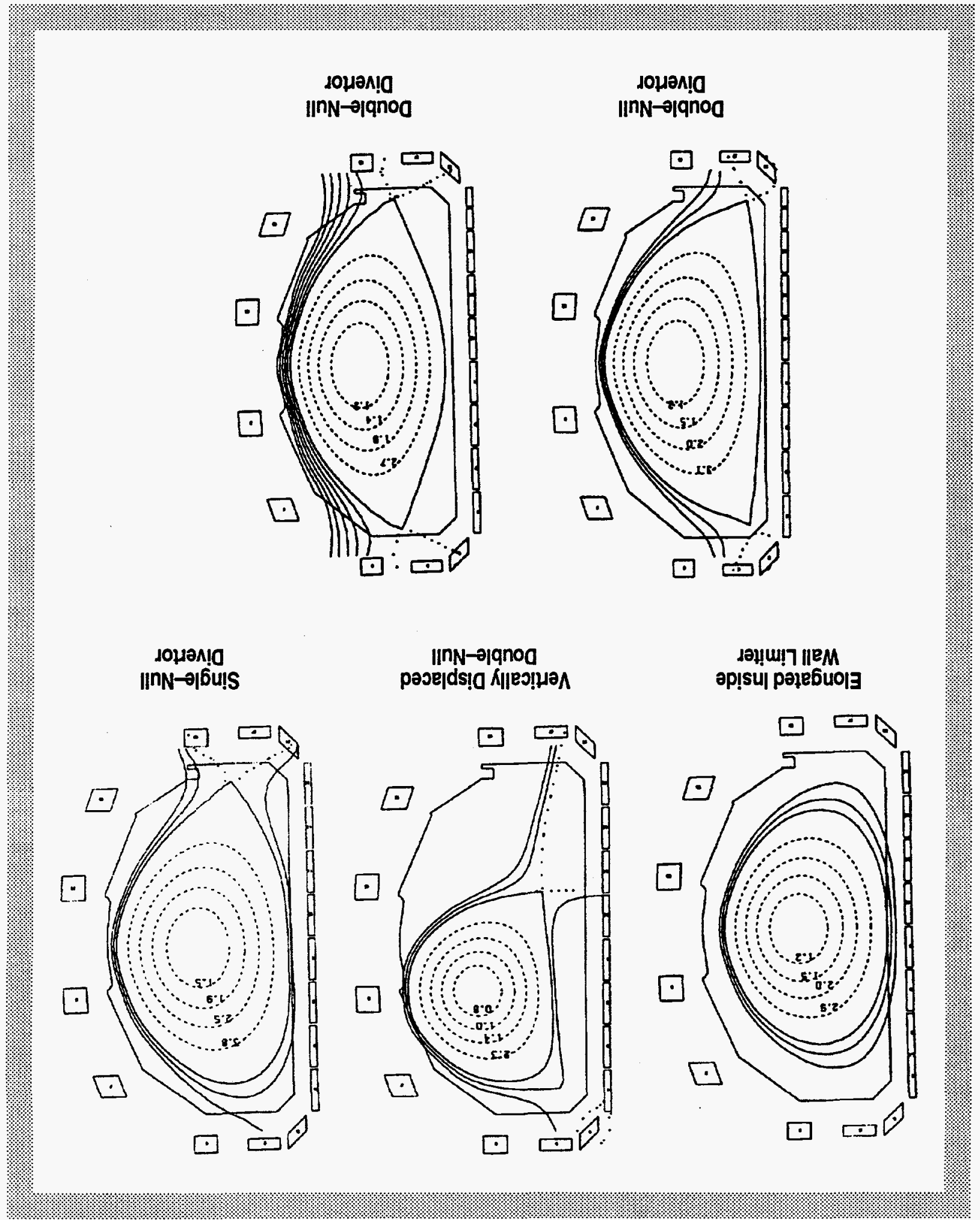




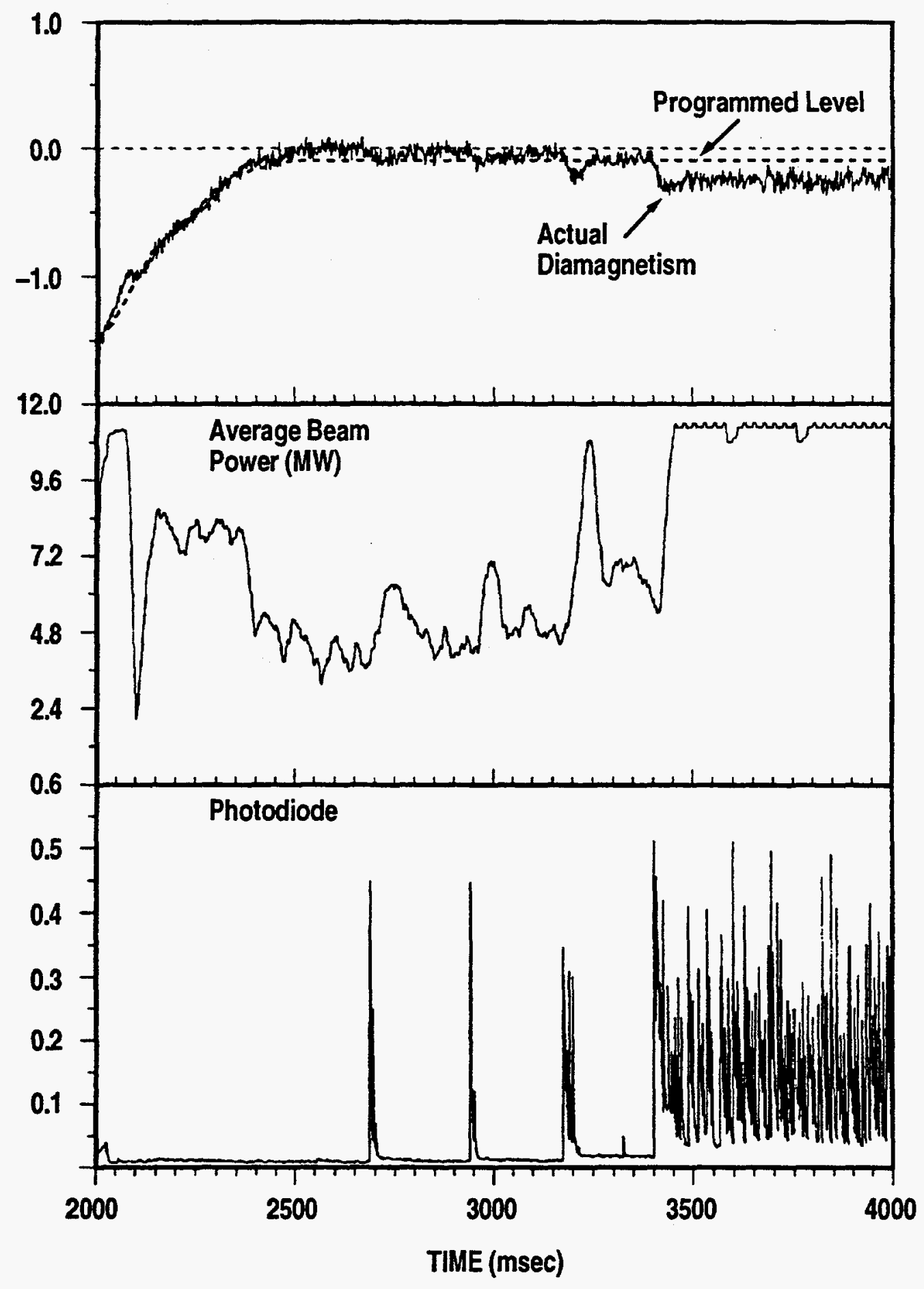

Fig. 5.4.-2. Controlling plasma stored energy with the advanced control system. 
the load resistance from antenna forward and reflected voltage diagnostic measurements and adjusted the gap between the antenna and the plasma in order to keep the load resistance constant.

The printed circuit boards for the digital control system circuits have been released for fabrication and will be available for use in the control system when operation is resumed in FY93. The digital data acquisition and processing hardware developed by the DIII-D team for use in the advanced control system also has applications in the realtime reduction of diagnostic data [5.4-2,5.4-4]. This is useful for diagnostics in present experiments that produce too much data to store during a shot or for long-pulse or steadystate tokamak experiments where data are produced continuously. A good example in present experiments is the set of Mirnov probes, which produces tremendous amounts of data in a short time through high frequency sampling of the diagnostic signals. A proof-ofprinciple software development project demonstrated that the available hardware had the computing power for realtime spectral analysis of two Mirnov probe signals [5.4-4]. This realtime analysis could be used to provide a continuous record of the frequency and toroidal mode number of the highest amplitude peaks in the frequency spectrum.

\section{REFERENCES FOR SECTION 5.4}

[5.4-1] Ferron, J.R., et al., in Fusion Engineering (Proc. 14th Symp. San Diego, 1991) Vol. 2, IEEE, New York (1992) 761.

[5.4-2] Ferron, J.R., Rev. Sci. Instrum. 63 (1992) 5464.

[5.4-3] Campbell, G.L., et al., "New DIII-D Tokamak Plasma Control System," submitted to Fusion Engineering (Proc. 17th Symp. Rome, 1992).

[5.4-4] Ferron, J.R., and E. J. Strait, Rev. Sci. Instrum. 63 (1992) 4799. 


\subsection{DIAGNOSTICS}

\subsubsection{OVERVIEW}

The DIII-D diagnostic effort in FY92 was aimed at continued improvement and expansion of the divertor diagnostic set and strengthening of profile and fluctuation measurements. Ten divertor or boundary physics diagnostic projects were either completed or initiated during the year. These diagnostic improvements will greatly enhance our ability to understand and exploit the new pumped divertor. Major upgrades to the multipulse Thomson scattering system and the motional Stark effect (MSE) diagnostic along with new fluctuation diagnostics will play a key role in the continuing effort to improve and understand the advanced tokamak plasmas developed on DIII-D. There are 41 major diagnostic systems operating on DIII-D with an additional nine diagnostic systems under development or undergoing major improvements. Table 5.5-1 lists the major diagnostic systems along with the quantities measured. As can be seen from the table, many of the diagnostic systems involve national or international collaborative efforts. The diagnostics that are built and operated in conjunction with collaborators have become an essential element in the successful operation of the DIII-D program.

\subsubsection{NEW OR UPGRADED DIAGNOSTICS}

In FY92, a number of improvements and upgrades to existing systems were completed and a large number of new diagnostics projects were initiated, principally in the area of divertor diagnostics.

A $\mathrm{Li}$ beam diagnostic became operational in FY92 and represents a major addition to the fluctuation measurement capabilities on DIII-D. A high-speed magnetic probe array was installed and produced magnetic fluctuation data with better frequency response and spatial resolution then was previously available. The data from the fast magnetic probes has been useful in a number of stability experiments.

The multipulse Thomson scattering system was upgraded to include eight lasers each operating at $50 \mathrm{~Hz}$. The system now provides a complete 40-point electron temperature and density profile every $6.25 \mathrm{msec}$ during the entire plasma pulse. This has greatly improved the efficiency of tokamak operations since there is less need for repeat plasma discharges to get time-dependent kinetic profiles. In collaboration with Lawrence Livermore National Laboratory (LLNL), an upgrade to the MSE diagnostic that measures the poloidal magnetic 
TABLE 5.5-1

PLASMA DIAGNOSTICS

\begin{tabular}{|c|c|c|}
\hline Diagnostic & Quantity Measured & Comments \\
\hline \multicolumn{3}{|c|}{ Operations-Related Diagnostics } \\
\hline Magnetics & $\dot{B}$, Magnetic flux, $I_{p}$ & $\begin{array}{l}2 \text { poloidal (58), } 1 \text { toroidal (8) mag. probe arrays with } \\
50 \mathrm{kHz} \text { response; } 41 \text { flux loops, } 9 \text { diamagnetic loops, } \\
30 \text { saddle loops, } 3 \mathrm{I}_{\mathrm{p}} \text { Rogowskis }\end{array}$ \\
\hline Hard X-rays & & 2 toroidal locations, 4 detectors, $1 \mathrm{kHz}$ response \\
\hline Plasma TV & Visible TV & Radial view, $180^{\circ}$ limiter, divertor \\
\hline IR cameras & Heat load to armored surfaces & $\begin{array}{l}180^{\circ} \text { limiter, upper } \\
\text { divertor; inside wall lower divertor (LLNL) }\end{array}$ \\
\hline$H_{\alpha}$ monitors & $H_{\alpha}$ radiation, recycling & $H_{\alpha}$ filtered, 16 locations, $10 \mathrm{kHz}$ response \\
\hline Neutron detectors & Fusion and photo-neutrons & 3 toroidal locations, $200 \mathrm{~Hz}$ response \\
\hline Soft $X$-ray arrays & Internal fluctuations & $\begin{array}{l}1 \text { vertical, } 1 \text { horizontal, } 32 \mathrm{ch} \text {. ea. }>250 \mathrm{kHz} \text { response, } \\
\sim 4 \mathrm{~cm} \text { resolution, } 4 \text { toroidal locations, } 12 \mathrm{ch} \text {. ea. } \\
100 \mathrm{kHz}\end{array}$ \\
\hline
\end{tabular}

\section{Electron Profiles}

$\begin{array}{ll}\text { ECE grating radiometer } & T_{e}(r, t) \\ \text { Multipulse Thomson profile } & T_{e}(r, t), n_{e}(r) \\ \mathrm{CO}_{2} \text { interferometers } & \bar{n}_{e} \\ \text { ECE Michelson } & T_{e}(r, t)\end{array}$

Radial profile, 10 ch., 0.1 msec

8 lasers, $6 \mathrm{msec}$, vertical profile, 40 pts., $<1 \mathrm{~cm}$ in edge resolution, $20 \mathrm{keV}>T_{e}>10 \mathrm{eV}$

Vertical, 3 chords; radial, 1 chord

Radial profile, each $25 \mathrm{msec}$ 
TABLE 5.5-1 (Continued)

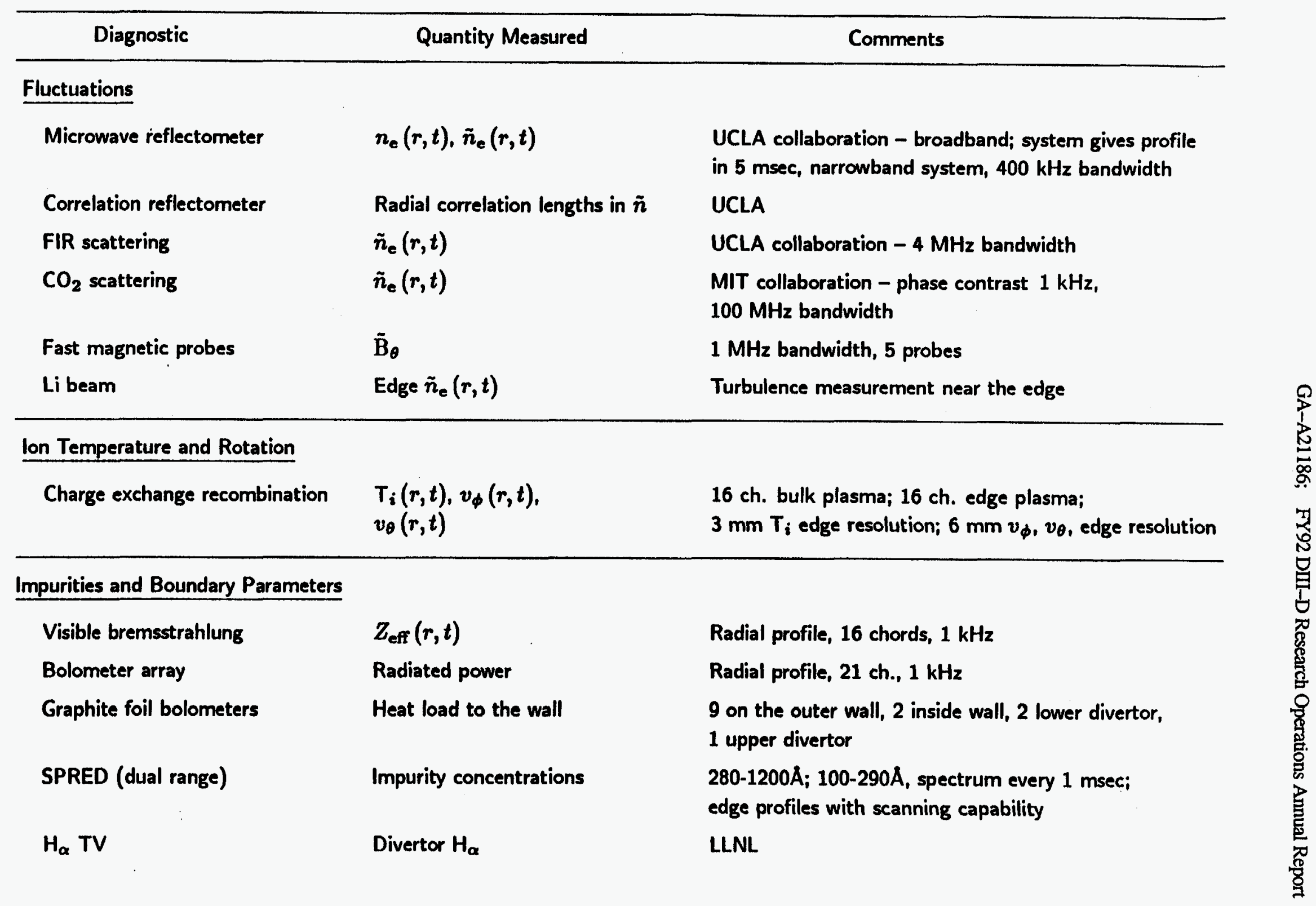


TABLE 5.5-1 (Continued)

\begin{tabular}{|c|c|c|}
\hline Diagnostic & Quantity Measured & Comments \\
\hline \multicolumn{3}{|c|}{ Impurities and Boundary Parameters (Cont.) } \\
\hline Divertor IR camera & Heat load to divertors & LLNL, GA, 125 msec for a profile \\
\hline Langmuir probes & Edge $T_{e}(t), n_{e}(t)$ & On divertor tiles \\
\hline Laser blowoff system & Impurity injection & Impurity transport \\
\hline Fast stroke Langmuir probe & Edge $T_{e}(t), n_{e}(t)$ & SNL, UCLA \\
\hline DIMES & Surface erosion & Divertor Material Exposure System \\
\hline Penning gauges & He pressure & ORNL - He pressure under the divertor baffle \\
\hline Fast pressure gauges & Neutral pressure & Near divertor region (ASDEX, LLNL), 2-3 msec response \\
\hline Tile current monitors & Poloidal tile currents & Includes tile currents in the ADP \\
\hline $\begin{array}{l}\text { Multichannel divertor visible } \\
\text { spectrometer (MDS) }\end{array}$ & Divertor impurity concentrations & ORNL - $7 \mathrm{ch}$. \\
\hline Divertor Baratron gauge & Neutral pressure & ORNL - verified ASDEX gauge measurements \\
\hline
\end{tabular}

\section{Fast Ion Diagnostics}

\section{Scintillator \\ E\|B charge exchange}

Fusion products

\section{Neutron fluctuations}

Beam-ion density profile

Fast neutrons, tritons

$\mathrm{He}^{3}$, other fast ions

\section{$50 \mathrm{kHz}$ response time}

Conventional charge exchange analyzer coupled with $H_{\alpha}$ detectors used to measure beam neutral density profile, scannable, spectrum in $1 \mathrm{msec}$

$\mathrm{UCl}$ collaboration; movable probe assembly 
TABLE 5.5-1 (Continued)

\begin{tabular}{|c|c|c|}
\hline Diagnostic & Quantity Measured & Comments \\
\hline \multicolumn{3}{|l|}{ Current Drive Diagnostics } \\
\hline Motional Stark effect (MSE) & $\mathrm{B}_{\mathrm{p}}(r)$ & 6 radial ch.; $2 \mathrm{~cm}$ resolution, $5 \mathrm{msec}$ response \\
\hline ECE Michelson & Microwave emission & $\begin{array}{l}\text { Detects tail population in the electron distribution } \\
\text { function, profile in } 25 \text { msec (LLNL and Maryland) }\end{array}$ \\
\hline SXR pulse height & High energy $X$-ray spectrum & $\begin{array}{l}\text { Russian collaboration; detects tail population in } \\
\text { electron distribution; spectrum every } 100 \mathrm{msec}\end{array}$ \\
\hline RF probes & RF radiation & Phase and amplitude of ICH of on inside wall \\
\hline \multicolumn{3}{|l|}{ In Development } \\
\hline Graphite foil bolometer & Radiated power & 2 poloidal arrays - 20 ch. ea., $1 \mathrm{kHz}$ \\
\hline ADP RGA & Neutral gas mass spectrometer & ADP physics RGA (ORNL) \\
\hline Second lower divertor IR TV & Heat load to divertor & Designed to measure toroidal asymmetries (LLNL) \\
\hline $\begin{array}{l}\text { Second fast magnetic } \\
\text { probe array }\end{array}$ & $\tilde{\mathbf{B}}_{\boldsymbol{\theta}}$ & $1 \mathrm{MHz}$ bandwidth \\
\hline Toroidal fast pressure gauges & Neutral pressure & Toroidal pressure asymmetries (ASDEX, ORNL) \\
\hline Toroidal tile current monitors & Tile current & Complete toroidal array in the divertor \\
\hline Upper divertor $\mathrm{H}_{\alpha}$ monitor & Visible radiation & Expanded array ( $8 \mathrm{ch}$.) viewing upper divertor \\
\hline
\end{tabular}


field $B_{p}$ was completed in time for the FY92 operation campaign. The upgraded system now has six spatial channels, provides toroidal plasma current profiles for each shot and has greatly improved the quality of the magnetic equilibrium calculations.

In the rf area, a reflectometer incorporated into the ICH antenna has been installed in collaboration with ORNL and the University of California at Los Angeles (UCLA). This new reflectometer will be used to study the local density profile directly in front of the antenna. An rf probe array has been installed on the inside wall of the torus directly across from the fast wave (FW) antenna in order to determine the current drive efficiency.

The boundary physics diagnostic set was improved with the addition of a set of Penning gauges that will be used to measure the He pressure under the divertor baffle plate. These gauges were installed in collaboration with ORNL and will be used in He transport studies in FY93. Plasma surface interactions in the divertor region will be studied in FY93 with the newly installed DIvertor Material Exposure System (DIMES). In conjunction with the dimes installation, and in collaboration with ORNL, the divertor spectrometer that views the area around the DIMES was upgraded to seven spatial channels.

The visible spectrometer brought to GA for divertor studies under the ORNL collaboration has been converted into a multichord system viewing seven different locations simultaneously in the lower divertor. As shown in Figs. 5.5-1(a) and 5.5-1(b), the collection optics provide 15 possible view chords arranged in two orthogonal vertical fans: one in the poloidal plane, the other in the toroidal plane. Each view chord intercepts a $2 \mathrm{~cm}$ diameter circle on the vessel floor, with the central chord in the crossed array aligned with the sample head of the DIMES. Light from the collection lens on the tokamak is conveyed via monofilamentary quartz fiber guide optics outside the biological shield wall to the entrance slit of the Czerny Turner spectrometer.

This multichord diagnostic became operational in May 1992, near the end of our experimental campaign for the year. For the poloidal array of seven view chords centered on the DIMES port, survey data was accumulated for the entire spectral range $350-720 \mathrm{~nm}$ by shot-to-shot scanning of the central wavelength. Spectral lines were identified from neutral and low charge states of $\mathrm{D}, \mathrm{He}, \mathrm{B}, \mathrm{C}, \mathrm{O}, \mathrm{Cr}, \mathrm{Ni}$ and a pair of current drive molecular bands. Utilizing radial scans of the $\mathrm{X}$-point position, the intensity distributions of the various atomic and molecular species were mapped relative to the intersection points of the separatrix with the floor; marked differences in radial distribution were observed. 
Fig. 5.5-1. The fan of view chords in the poloidal plane spans the interaction region with the vessel floor of the single-null divertor configuration. (b) The solid black circles show the locations of the 15 fiber optic views on the concentric rings of graphite floor tiles; the central circle in the crossed pattern intersects the head of the DIMES probe. (a)

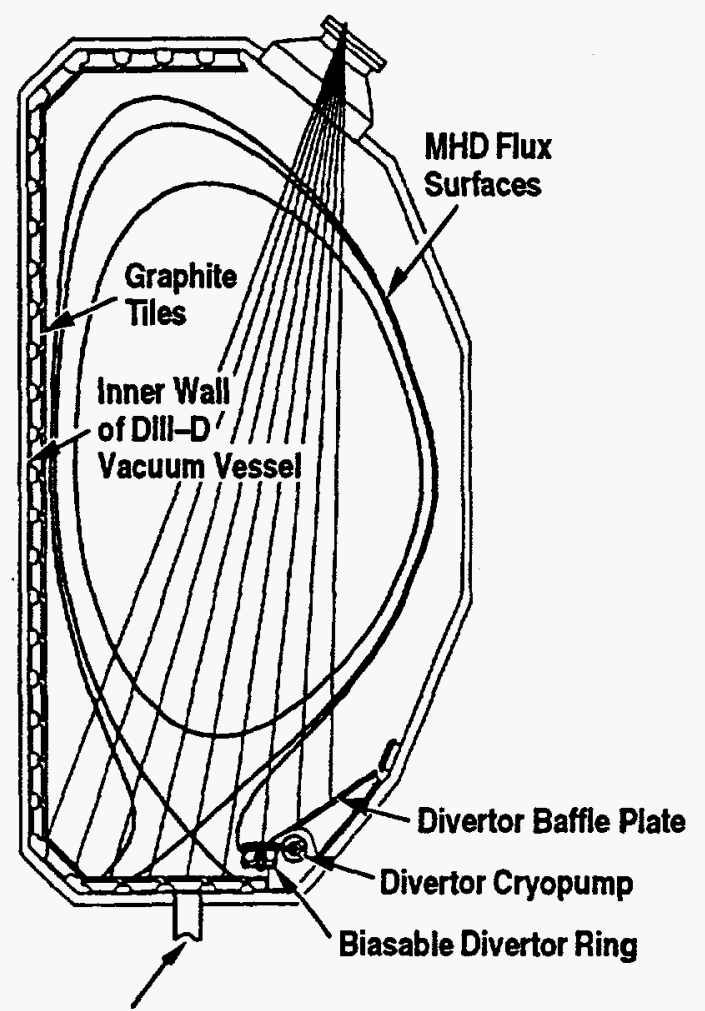

Port Containing DIMES Probe

(b)

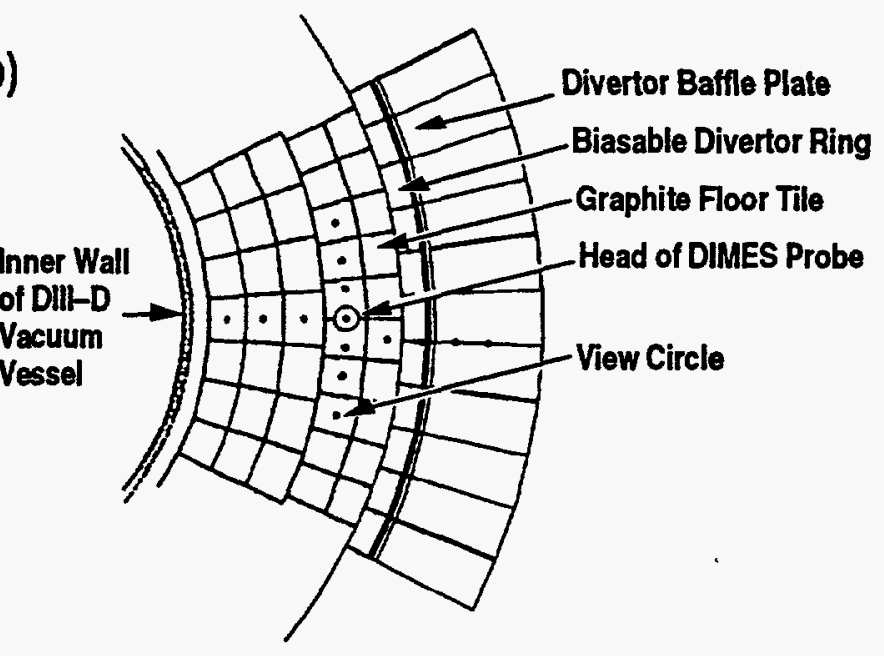


GA-A21186; FY92 DII-D Research Operations Annual Report

The Phase Contrast Interferometer, became operational on DIII-D in early 1992. This diagnostic produces a spatial image of the density fluctuations near the outer edge of the plasma with high speed and excellent radial resolution. Long wavelengths, up to $7.6 \mathrm{~cm}$, can be resolved. Data were collected throughout the 1992 campaign in a wide variety of experimental conditions. The analysis of those data is underway and is already providing new insight into the phenomenology of the $\mathrm{L}-$ to $\mathrm{H}$-mode transition and of the edge localized modes (ELMs).

\subsubsection{DIAGNOSTICS UNDER DEVELOPMENT}

Our FY93 diagnostic plans emphasize expanding and improving the divertor diagnostics both for the new pumped divertor and in anticipation of the planned radiative divertor. Two new bolometer arrays will be completed and installed in early FY93. New graphite foil bolometers will be installed in the divertor and the inside wall. These diagnostics will improve our ability to determine radiated power profiles, particularly details of the radiated power from the divertor region.

Toroidal asymmetries associated with divertor operation will be addressed with a new toroidally separated divertor infrared (IR) camera (LLNL), additional fast pressure gauges (ORNL), and an extensive toroidal tile current array. Depending on funding levels, additional divertor diagnostics are planned. A more complete list of diagnostics under development can be found in Table 5.5-1.

An upgraded, eight-channel, MSE diagnostic was built and installed on the DIII-D tokamak. The local magnetic field tilt angle in the plasma interior is deduced from the polarization direction of $D_{\alpha}$ line emitted by one of the heating neutral beams. Thorough calibrations of the diagnostic were conducted, with special emphasis on the effect of the tokamak magnetic field on the polarimeter optics and on the measured magnetic field tilt angle. The diagnostic was operated routinely during the spring/summer campaign of 1992 and the MSE data were used in the analysis of current profiles for a variety of plasma discharges including VH-mode, current ramp, and elongation ramp experiments.

A study of sawtoothing discharges indicated that the value of central safety factor is usually close to 1.0. This result is in an apparent disagreement with the results obtained previously with a single channel system where $q_{0}$ " 1 (e.g., 0.7 ) was deduced for low-q discharges. The discrepancy is attributed to a better accuracy of the current 
profile reconstruction possible with the presently available multiple measurements in the plasma center.

\subsection{RELIABILITY AND AVAILABILITY}

\subsubsection{INTEGRATED PREVENTIVE MAINTENANCE PROGRAM}

The DIII-D Project implemented an Integrated Preventive Maintenance Program (IPMP) in March 1991 which put all equipment requiring preventive maintenance into a common computerized database. The IPMP used a database program named MAINTSTAR developed by Bender Engineering Inc. In 1992, the preventive maintenance database was transferred from MAINTSTAR to a GA Fusion-developed software program called PACMAIN. PACMAIN will provide easier access to preventive maintenance data by Fusion personnel via their VAX terminals, thus reducing the number of hard copy reports currently being generated and distributed. Software support will be provided in a more timely manner. Also, the customization of reports will be made a lot easier.

During this reporting period, two additional technical groups (RF Systems and Safety) have started to utilize the computerized preventive maintenance program. The list of systems now integrated into the program includes:

- Vacuum Systems.

- Water Cooling Systems

- Air Systems.

- Cryogenic Systems.

- Prime Power/Motor Generator (MG)

- B, E, \& F Coils Power Systems.

- Neutral Beamlines Power Systems.

- Neutral Beamlines Mechanical.
- Diagnostics.

- Mechanical Systems.

- Electronic Systems.

- Operations.

$\therefore$ Facilities.

- RF Systems (added).

- Safety (added). 
At the start of FY92, there were 861 pieces of equipment in the IPMP database. Equipment is continuing to be entered into the database, and as of the end of FY92 there are 1079 pieces of equipment in the IPMP; an increase of 25\%. During FY92, 2900 work orders were issued or an average of 242 per month. Of these, 513 were not completed due to equipment not operating during the vent period. At this point, the bulk of the obvious DIII-D equipment has been entered into the IPMP. Equipment will continue to be entered though at a reduced rate from the previous two years.

The last major group of equipment, which is presently being entered into the IPMP database, is cyclic dependent equipment such as valves, shutters, bellows, etc. The IPMP will issue a work order for the procurement and/or fabrication of cyclic dependent equipment prior to their end-of-life estimates. Installation of the equipment can then be completed without any disruption of the DIII-D machine operation. Additionally, critical end-of-life actuals can now be recorded and maintained for future use.

\subsubsection{SIGNIFICANT EVENT REVIEW}

The purpose of the Significant Event Reviews (SERs) is to learn from past events with the objective of determining the root cause of problems and, thus, eliminate the reoccurrence of those events which result in unanticipated costs or expended effort which could have been avoided with better design, procedures, etc. The SER brings together key personnel involved in the event with key people from related parts of the organization to discuss the event and propose courses of action. Further, the SER addresses the application of the proposed solutions to similar types of activites in other systems. The SER is followed up with a Significant Event Report.

There have been seven SERs held during FY92 on the following issues:

1. RF bellows failure.

2. Advanced Divertor Program (ADP) ring inductor support stand collapse.

3. Plasma operations shutdown due to false smoke detector signal.

4. Overpressurization of the $150^{\circ}$ Left neutral beam ion source.

5. ICRF stub tuner failure. 
6. Debonding of boron carbide coating on FWCD antenna Faraday shield.

7. Proposed use of pure oxygen in DIII-D gas system.

The course of action followed for these SERs resulted in changes to documentation such as procedures, drawings, check lists, etc., to reduce the likelihood of future reoccurrences.

\subsection{RADIATION MANAGEMENT}

The total neutron radiation at the site boundary for FY92 was 2.8 millirem, the total gamma radiation was 1.4 millirem, giving a total site dose for the year of 4.2 millirem. [This is below the Department of Energy, San Francisco Operations Office (DOE SAN) annual guideline limit of 20 millirem and the California annual limit of 500 millirem)]

The total dose exposure personnel received was kept below the DIII-D procedural limits of 25 millirem per day, 100 millirem per week, and 300 millirem per quarter (1200 millirem per year). The highest dose accumulated by an individual from pit runs and vessel entries (but not operations) for FY92 was 365 millirem. A total of 129 individuals received such doses as follows:

\begin{tabular}{cccc}
\hline $\begin{array}{c}\text { Dose Range } \\
\text { (millirem) }\end{array}$ & $\begin{array}{c}\text { Number } \\
\text { of People }\end{array}$ & $\begin{array}{c}\text { Dose Range } \\
\text { (millirem) }\end{array}$ & $\begin{array}{r}\text { Number } \\
\text { of People }\end{array}$ \\
\hline $0-25$ & 104 & $201-250$ & 0 \\
$26-50$ & 11 & $251-300$ & 0 \\
$51-100$ & 9 & $301-350$ & 2 \\
$101-150$ & 2 & $351-400$ & 1 \\
$151-200$ & 0 & & \\
\hline
\end{tabular}

All doses were logged in the database of personnel radiation doses.

The periodic (every two years) survey of the site boundary radiation continued. There were no unexpected differences from past measurements. 
GA-A21186; FY92 DIII-D Research Operations Annual Report

The annual radiological safety audit of the DIII-D facility was conducted by the manager of GA Health Physics. Nineteen action items were identified and all but one were completed in a timely matter. The outstanding action item concerns renewal of a work authorization.

Two radiation safety training classes were held for new personnel.

Preliminary analysis of shielding requirements to obtain a factor of 10 additional shielding for use in future upgraded operations was made. The results indicated that an additional 8 in. of poly would be needed. A detailed plan would also require structural analysis.

The DIII-D work authorization (WA) was reviewed, the radiation procedures were modified slightly, the authorized users list was updated, and the completed WA was approved by GA Health Physics. One improvement incorporated in the new WA is the ability to set up a hot machine area in the Building 34 machine shop instead of being restricted to the diagnostics lab.

The vessel was vented five times: (1) for 50 days starting October 31 , (2) for one day on January 16, (3) for six days starting February 27, (4) for ten days starting June 29, and (5) for the vent starting August 17 (45 days in the fourth quarter) which continues into next year. Radiation monitoring was performed for all vessel entries.

A large number of items removed from the machine pit have been checked for activation, and those with radiation levels above background are in storage. A barrel of waste trash and a barrel of contaminated oil were picked up by the waste yard.

During the February vent entry, four tritium wipe samples of the carbon tile surfaces were taken for the first time. The limiter read high $\left(140,000 \mathrm{DPM} / 100 \mathrm{~cm}^{2}\right)$ compared to the other surfaces (up to $2200 \mathrm{DPM} / 100 \mathrm{~cm}^{2}$ ). The higher level of tritium on the limiter was surprising but may be due to beam target interactions from impinging beam ions. A survey of tritium inside the vessel was performed during the July vent. A total of 24 wipe samples were taken from various locations including: wipes from the carbon tiles on the floor, ceiling, inner wall, outer wall, and limiters, and wipes of the Inconel outer wall. Results ranged from 100 to $6200 \mathrm{DPM} / 100 \mathrm{~cm}^{2}$. The highest concentrations of tritium were on the limiters followed by the carbon tiles on the outer wall. 
The carbon tiles removed from the machine during the August vent for cleaning were sampled for activation, removable alpha, removable beta, and removable tritium. The samples were taken from the approximately 1600 tiles at a sampling rate of 1 sample for every 10 tiles. The tiles showed no radiation above background for the activation, removable alpha, and removable beta. The 173 tritium samples had contamination levels on 163 tiles of less than $500 \mathrm{DPM} / 100 \mathrm{~cm}^{2}$, and on 10 tiles the contamination levels ranged up to $2500 \mathrm{DPM} / 100 \mathrm{~cm}^{2}$. Califomia regulations allow a release for unrestricted use for levels below $4000 \mathrm{DPM} / 100 \mathrm{~cm}^{2}$, but the DOE release limit is $1000 \mathrm{DPM} / 100 \mathrm{~cm}^{2}$; the DOE limit is followed.

\subsection{ELECTRICAL ENGINEERING}

\subsubsection{OVERVIEW}

Electrical engineering support of the DIII-D experimental program continued routinely throughout the operating period. Significant progress was made in implementing the new Advanced Plasma Control System for the DIII-D tokamak. Initial operation with prototype circuit cards in this plasma control system started just prior to the last shutdown period for the year and showed very encouraging results. Several upgrades were made to the electrical power systems. The most noteworthy of these were the upgrade of the control circuits for one of the dc power supplies used in the chopper system (D1-supply), installation of a new uninterruptible power supply (UPS) for the toroidal and poloidal field power supply controllers, and the upgrade of the control circuits for the toroidal field (B) power supply. Other electrical engineering activities during this period centered on preventative maintenance of transformers and switchgear components which require power shutdown for access.

\subsubsection{OPERATION SUPPORT}

A comprehensive study was performed to establish the present operational limits of the DIII-D electrical systems. Upgrades needed to the electrical systems to support future long-pulse experiments were identified and costed. This data was used in preparing the new DIII-D Long Range Plan.

Several cost-saving measures were also implemented during the year. A new startup schedule for the motor generators used to power the coil power supplies results in significant savings in power demand charges. A plan to install four new power supplies (from LLNL) to be 
used in the $\mathrm{n}=1$ coil and future $\mathrm{C}$-coil systems has been approved. Work was started to remove/relocate some power system components in the poloidal power supply area in order to make room for the new power supplies. These power supplies will arrive during the first quarter of FY93.

\subsubsection{COIL POWER SYSTEMS}

Failure reports and associated downtime records were used in planning upgrades to the electrical systems. These records provided information which allowed significant improvements in the operation of the D1 and B power supplies which had been the main contributors to the electrical system downtime.

\subsubsection{HIGH VOLTAGE SYSTEMS}

Many of the high-power tetrode vacuum tubes used in the modulator regulator subsystems of the neutral beam power supplies are approaching the end of their useful service life. We have identified a vendor who may be able to rebuild these tubes at substantial savings. Two tubes are presently being rebuilt and will be tested as soon as we receive them back from the vendor.

Modifications to the control and protection circuits for the ECH No. 1 high-voltage power supply were completed, and this power supply can now be used for both the 60 and $110 \mathrm{GHz}$ gyrotron systems. This modification enables operation of both the $\mathrm{ECH}$ gyrotron systems while all eight beam systems are being used. Previously, one beam power supply had to be converted to negative polarity operation in order to support $\mathrm{ECH}$ experiments.

\subsubsection{AC SYSTEMS}

The extended shutdown periods enabled maintenance of switch gear and transformers that are normally in service. The 138 and $69 \mathrm{kV}$ oil circuit breakers were disassembled, cleaned, adjusted, and placed back in service. The $138 / 12.47 \mathrm{kV}$ transformer oil was processed to remove sludge. The roof of the $4160 \mathrm{~V}$ switchgear was replaced to prevent moisture from entering the enclosure. Transformers T9 and T11 were oil processed after finding unacceptable levels of combustible gas in their oil samples. Two transformers (T2 and T3) were found to contain excessive PCB amounts. These were oil processed by a PCB removal certified contractor to meet the acceptable criteria ( $<50 \mathrm{ppm}$ PCB content). Maintenance of the high current switches was started and will be completed before the startup of operation. 
Ground fault detectors were installed in all the major motor feed circuits to avoid tripout of the main circuits when motor ground faults occur. The fifth Toshiba motor drive for the $\mathrm{ECH}$ water pump motors was installed and tested. Control wiring of a new reduced voltage motor starter for the Sullair Helium Compressor was completed and tested.

\subsubsection{INSTRUMENTATION AND CONTROL SYSTEMS}

Several new diagnostic, vacuum, and cryogenic system-related tasks were completed by the instrumentation and control system group:

1. Thomson Profile System. Design and fabrication of various electro-optical systems.

2. Li-Beam System. Design of a low noise amplifier for the Li-beam diagnostic system was completed.

3. Vacuum and Cryogenic Systems. All of the solenoids for the valves in the vacuum control system were replaced and moved to a central location for improved reliability and ease of maintenance. The ADP cryostat was instrumented and tested. Instrumentation and control interfaces were designed and installed for the new $\mathrm{He}$ liquifier and Sullair compressor.

\subsubsection{PREVENTIVE MAINTENANCE}

The electrical systems are now fully embraced by the preventive maintenance (PM) program, and 1128 PM tasks were completed in FY92. Major preventive maintenance tasks were performed on transformers, switchgear, and power systems as previously described.

\subsection{MECHANICAL ENGINEERING}

\subsubsection{TOKAMAK SYSTEMS}

\subsubsection{Outer Wall Tile Upgrade - Limited Installation}

A limited series of graphite and Inconel tiles was installed on the outer wall of the vessel in December 1991. These tiles are intended to support machine operations for the remainder of the fiscal year while the project to place tiles on the entire outer wall surface 
is scheduled for completion by the end of calendar year 1992. A total of 291 new tiles were required for the limited series. This included 277 graphite and 14 Inconel tiles fabricated in $\mathbf{4 7}$ different styles to account for the numerous special needs and irregularities of placement on the vessel wall.

\subsubsection{Fast Wave Current Drive Antenna}

In February 1992, one of the inner conductors feeding the No. 2 current strap, internal to the antenna, was determined to have failed due to a local concentration of high current at one of the internal bellows. The machine was vented, and the antenna was partially disassembled to effect repair/replacement of the damaged bellows. The entire procedure was completed in less than two days thus creating minimal impact on both the vessel and subsequent machine operations.

\subsubsection{Support of Neutral Beam Operations}

For the past year, mechanical engineering support has been provided to the neutral beams operating group. This included internal inspections of the beamlines for mechanical problems, analysis of accumulated residue material found in the lines, plus followup of fabrication and procurement of hardware and equipment for the beam system. Engineering support was also provided to assist with repair, redesign, and reinstallation of the ion sources due to a mechanical failure attributed to an over pressurization and the use of nonspec parts in the original mechanical supports for these sources.

\subsubsection{Engineering Work Procedures - Tokamak Operations Division}

Development of engineering work procedures for use within the DIII-D has been in progress for the past year. These procedures cover policies by which projects can be initiated, controlled, and implemented to support the DIII-D tokamak and its associated systems. A total of eight procedures have been completed.

\subsubsection{Correction Coil for the Tokamak}

Studies were completed to evaluate the scope of work and conceptual design for a new correction coil system (C-coil) to replace the existing correction coil ( $\mathrm{n}=1$ coil). These coils would be oriented in a vertical plane and would each cover a $60^{\circ}$ sector toroidally 
around the vessel. The project would be quite complex due to the myriad of diagnostics and interferences located near the vessel. Budgets and schedules have been prepared to support this project which is under consideration for future funding.

\subsubsection{B-Coil Prestressing}

The vertical prestress loads on the B-coils were checked early this year to measure the level of relaxation that had occurred since the original installation several years ago. Virtually all of the 48 prestress loads were found to be out of specification. A program was established to reset these loads and to develop a procedure for incorporation into the preventive maintenance program so that these loads can be monitored on a routine basis in the future.

The horizontal prestress on these coils was checked and reset last fiscal year. In keeping with the ongoing program to monitor key elements of machine performance, a procedure was developed to perform a reset of these prestress loads as well. The procedure and the routine performance of this task has also been incorporated into the DIII-D PM program.

\subsubsection{Mechanical Stiffening of the B-Coil Power Feed Point}

Deflection measurements were taken during machine operation to determine the amount of flexure occurring at the $30^{\circ} \mathrm{B}$-coil power feed point. From these data, the developed forces applied to this point during routine machine operation could be determined. A support structure was then designed to provide the correct amount of stiffening to prevent excessive movement, flexure, and loosening at this feed point.

Temperature measurements taken in conjunction with the flexure data indicated that a need also exists to provide water cooling in the connecting elements at this point. The bus bars have been modified to provide cooling paths for connection to the facility cooling water system.

\subsubsection{Removal of the $180^{\circ}$ Pumped Limiter}

The pumped limiter assembly located on the vessel midplane at $180^{\circ}$ has been removed from the vessel. This was completed in support of the installation of a second FWCD antenna planned for the next fiscal year. Removal was effected via written procedure and was executed without problem. The port area has been reworked and modified to accommodate a new port flange designed to accept installation of the new antenna. 


\subsubsection{Solenoid Valve Replacement - Air Operated Valves}

A new array of solenoid valves has been installed in the pit area to consolidate the many solenoid valves previously scattered at various locations near the air operated valves they serve. This also allows the air lines between the solenoids and the valves to be routed in organized groups for a more efficient installation. This work is currently in progress with completion expected early in FY93.

The existing solenoid valves that control the tokamak's vacuum valves and shutters are badly leaking and require replacement. A prototypic bank of solenoids was successfully tested. The solenoids were purchased and installation is in progress.

\subsubsection{B-Coil Belt Bus Joints}

The belt bus joint currently installed on DIII-D has been redesigned and prototype hardware has been procured. These new joints are scheduled for testing next year to evaluate capability and performance.

\subsubsection{Diagnostic Support Activities}

Mechanical Engineering continues to provide design and analytical support for a number of diagnostics.

1. New Bolometer Array. A development program has been carried out to evaluate insulating materials, signal connectors, and outgassing properties of the bolometer sensors. Final design drawings have been completed and fabrication of the parts is in progress as required to support installation during the vent period early next fiscal year.

2. High Frequency Magnetic Probe Array. A group of five magnetic probes has been designed and installed in the vessel at $150^{\circ} \mathrm{R}-1$. This array uses fine copper wire wound on a macor machinable ceramic core. Fabrication was completed and the array was tested for thermal cycling and vacuum integrity before being installed in the vessel. A second set of high frequency probes based on the same design is being fabricated for installation on DIII-D later in FY93. 
3. Lithium Beam Injector. Engineering support was provided to the physics program responsible for developing an operating lithium beam injector on DIII-D. Hardware elements of the injector system were designed and fabricated along with equipment supports capable of providing three-dimensional adjustment capability.

4. Modified Tile Current Monitors. Several prototype tile current monitors have been designed and fabricated for installation during the year-end vent. These monitors are intended to be an improvement on the existing installation. They draw current from two tiles and have been designed with a heavier support structure compared to previous designs.

5. Reflectometer (FWCD). In conjunction with the ORNL FWCD rf antenna, a small reflectometer array designed and fabricated by the ORNL rf physics group has been installed immediately adjacent to the existing antenna. The reflectometer interface to the plasma consists of four horns fabricated from graphite.

6. Toroidal Soft $\mathrm{X}$-ray_ $\mathrm{A}$ toroidal soft $\mathrm{X}$-ray diagnostic has been redesigned to accept the installation of a new 20 detector array. The diagnostic has been installed in the vessel in preparation for next year's operations program.

\subsubsection{FLUID SYSTEMS}

\subsubsection{New Water System}

Construction of the low-pressure cooling water system was completed and a successful hydrostatic test performed. Electrical service including controls and interlocks was connected to the two installed pumps and the pumps operationally checked out.

\subsubsection{Cooling Water Deionization}

Arrowhead, Polymetrics, and other companies will no longer regenerate the DIII-D demineralizers due to the copper ion content present on the resin. Reverse osmosis was selected as the most promising means to control the conductivity of the circulating cooling water. A small reverse osmosis system is currently in test. Alternate filtration methods as a means to control conductivity are also being investigated. 


\subsubsection{New Helium Liquefier}

Design and manufacture of the Sulzer liquefier continued on schedule. Delivery is scheduled on or before November 1, 1992. Installation and startup testing are expected to be completed in late January 1993.

\subsubsection{New Helium Compressor}

Installation and initial startup of the new 400 horsepower Sullair helium compressor was completed. The field service engineer from Sullair was present to perform initial startup. No significant problems were encountered. The new compressor is required as part of the cryogenic system upgrade to provide the needed capacity to operate the advanced divertor cryopump and the four neutral beam cryopanels simultaneously. This addition nearly doubles helium gas compression capacity.

\subsubsection{Increased Helium Gas Storage Capacity}

A 20,000 gal gaseous helium storage tank was received at DIII-D and installed. This tank will upgrade helium gas storage capacity by $50 \%$ and allow storage of the entire cryosystem helium inventory as gas during cryosystem shutdown periods. This will result in cost savings since helium boil-off gas will not have to be vented to atmosphere.

\subsubsection{6000 Turbopump Operation}

The failed Varian 6000 turbopump was rebuilt on site by Varian personnel. A defective bearing was found to be the cause of the pump failure and it showed evidence of having been heated to very high temperature.

\subsubsection{Boronization Program}

A helium purge system was installed on all DIII-D secondary vacuum spaces. This purge will enable the pressure in the secondary vacuum spaces to be controlled so that it will always be higher than the vessel pressure during the boronization process to ensure that no diborane gas will migrate from the vessel into the secondary space. 


\subsection{FY92 COMPUTER DATA SYSTEMS}

\subsubsection{DIII-D}

Fiscal year 1992 was busy for the DIII-D computer systems group. Work fell in the area of supporting operations, upgrading hardware, upgrading and improving existing software, and bringing new diagnostics on-line to enhance the operation of the tokamak. Some of the software tasks were performed to facilitate remote collaboration. Programming tasks initiated by other groups were also reviewed and support was given where there were specific needs - Thomson laser development and plasma control system.

Although the operations period was short, the number of diagnostics and amount of data taken per shot increased during the year. During FY92, 2353 shots totaling 84.64 Gbyte of data were taken. During the last regularly scheduled run period, a new largest shot size was recorded of 69.8 mbytes. The MODCOMP data acquisition computer load became significant and fueled the investigation into replacement of the existing systems.

\subsubsection{COMPUTER HARDWARE UPGRADES}

All VAX computers were upgraded to VMS 5.4-2 as was the latest FORTRAN compiler. Multinet communications software was also installed. The Multinet software is needed to support the access of DIII-D computers by the collaborators. A VAX 8650 was installed in the cluster and the VAX 11/785 moved to the User Service Center (USC). Six Systems Industries 1.3 Gbyte disks were acquired. Four of the new disks were installed at DIII-D and two were installed in the USC. The addition of those new disks provides a total of nearly 13 Gbyte of on-line shot data at DIII-D and USC. New high-density 5 Gbyte $8 \mathrm{~mm}$ tape drives were put into production during this period. They are being used to archive all of the DIII-D shot data files and for backup media. The USC VAX Cluster performance was enhanced with upgrades which give full Cluster functionality to the VAX4000-300s, reducing the length of time needed to boot and increasing the overall availability of the Cluster.

Part of the long-range fusion computing plan is to incorporate high powered workstations into areas of identified need. Three workstations were purchased from Hewlett Packard (HP) and one from Silicon Graphics (SGI). The HP workstations (all Model 730) will be used for general physics code development and computations. The SGI Indigo will be used on the Fokker Planck code CQL3D for if and neutral beam heating simulations. 
GA-A21186; FY92 DII-D Research Operations Annual Report

A SUN Unix system was added to the DIII-D local DECnet network. This SUN is collecting Langmuir data in a collaboration with SNL. Modifications were made to local VAX software, so that data generated on the SUN is now collected directly to the VAX and added to the shot database. This is a significant event, since it is expected that other types of CPUs will be desirable to use in the realtime environment.

Another goal of the long-range fusion computing plan is to make all devices in the LAN accessible through ethernet. In support of this goal, several network compatible printstations were procured from Talaris and installed on the LAN ethernet.

\subsubsection{SOFTWARE IMPROVEMENTS}

The neutral beam operating software was improved. The neutral beam systems now provide more capabilities to the operators and can have their parameters changed more quickly to meet the demands of the experimentalist from shot to shot.

All MODCOMP computers were upgraded to the latest operating system version K.02. InterCPU software was upgraded to the latest version, and the database system was upgraded to the latest version.

The acquisition system software was modified so that clock modules can be located on any CAMAC highway of the two CPUs. This required changes to the clock synchronization software. This gives us more flexibility in location of CAMAC modules on the various highways and allows for a more even spread of data amounts per CPU.

The TCP/IP protocol has been incorporated into the subroutine used to access DIII-D data, which now allows the data to be accessible to any UNIX workstation on the national network.

The USC assumed responsibility for the Cray code conversion task, either participating directly in the conversion or coordinating others in their efforts. This task was brought about by the decision made by NERSC to switch from the proprietary Cray operating system (CTSS) to the open system (UNICOS). All the major GA fusion production codes were converted by the USC assisted by temporary staff. The USC authored and will maintain FORTLIB, a library of routines which translates CTSS calls to UNICOS calls. The USC continues to provide local support for all NERSC Cray users, including maintenance updates to the Transport family of codes. 
The DIII-D staff was increased by one FTE, who is responsible for the computer system backups and for Macintosh support, including procuring and installing both hardware and software. Other DIII-D staff members are assisting with the Thomson diagnostic system and the plasma control system. Many changes have now been implemented in REVIEW, the major plotting and quick view program for DIII-D data. This code is now being maintained by the computer group.

\subsubsection{REMOTE ACCESS FOR OFF-SITE COLLABORATION}

The ability to perform routine experimental tasks from a remote location was implemented at DIII-D during this time. Network packages and local network software was added to the machines. Access of the data via the local subroutine was modified so that access can be done over long distances. LLNL SXR diagnostic was able to access DIII-D shot data between shots, run analysis on computers located at LLNL, and plot results on screens in the DIII-D control room. This is done quickly between shots and uses the computer resources which are supplied by the collaborator, with minimal overhead on the DIII-D systems.

Support is provided for the many collaborators currently visiting GA. The support extends from simple consulting to hardware installation and testing. A DECstation 3100 workstation from Oak Ridge was installed and integrated into the LAN for one of the ORNL collaborators. XTerminals have been installed for SNL and UCLA. The Interprocess Communication System (IPCS) from Livermore was installed on the VAXes both in the USC and at DIII-D for LLNL. IPCS is a message passing system that allows tasks distributed over a network to communicate with each other. It connects GA's VAXes with several of LLNL's computers. It is being used, in part, to analyze and display data at LLNL between tokamak shots at DIII-D.

A new version of the VAX networking software, Multinet, and the associated Client software, was installed. The Client software, which enables the VAX to mount disks from remote systems, was needed specifically for the IPCS. It also was used to mount the National Storage Lab (NSL) testbed. This is a prototype and demonstration testbed system at NERSC being developed to investigate technology and architecture for future storage systems.

\subsubsection{COMPUTER REPLACEMENT PLAN}

A project to investigate the feasibility of updating the existing MODCOMP systems for experimental control and data acquisition was begun. Various vendors were contacted and 
given a list of our proposed needs, all of which focus on open systems and standards. Vendors include Digital Equipment Corp., HP, IBM, MODCOMP, Harris, Encore and Sun Microsystems. Visits were made to the testing lab at NASA Langley, where a group of people have spent considerable time looking into open systems. CEBAF was also visited, since this is one of the few sites planning a new system from the ground up, based on currently available hardware. A detailed plan for system installation, software implementation, and costs was written. An request for quotation (RFQ) based on this plan was sent to seven vendors with five responding. In analyzing the responses, the major concerns were to have hardware and software which preserve our large investment in CAMAC modules and to purchase a computer which is upgradable and conforms to OPEN system standards. Hardware costs, software costs and development cost have also been considered. The decision to proceed with the acquisition has been made, and some equipment will be in-house during FY93.

A workshop was held with ORNL, Princeton Plasma Physics Laboratory (PPPL), LLNL, and GA. At issue are a common database so that the various codes (for example a transport code) can access GA tokamak data, and PPPL tokamak data, without having to know specific information about the file format where the data is stored. Another issue is the use of remote computers to collect tokamak data. This data may be scattered over many locations and be collected by different computers with different formats, and it needs to be gathered into a collection of files which are accessible on a national level. Many of the thoughts from this workshop will be included in the development of the new systems at DIII-D.

\subsubsection{PREVENTIVE MAINTENANCE TRACKING PROGRAM IMPROVED}

The USC assumed all support responsibility, including vendor contact, for the DIII-D maintenance tracking program, MAINTSTAR. A Novell network was installed between two maintenance stations allowing input from more than one terminal. Subsequent analysis of this system indicated that an in-house version running on the central computers under S1032 would be more cost efficient, allow for wider data access, allow for more timely problem response, give a greater user flexibility to the reports, and ensure backup. Thus, MAINTSTAR has been replaced by a similar program (PACMAIN) developed on the VAX. This has allowed a larger number of users to access the data than was possible with the old system. Interfaces to other DIII-D data, such as the TROUBLE database, are also planned. 


\subsubsection{NEW CAD SYSTEM BETTER SUPPORTS DIII-D}

Intense effort went into an upgrade of the existing Computervision (CV) CAD system at GA. A new CAD system was specified and bid, and all of the vendors were consulted. The system installed, which was most cost effective, was an upgrade of the existing CV systems to their new software (parametric) and SUN Sparc 2 hardware. The new CAD systems have also been integrated with the Fusion ethernet, thus making existing peripherals available to the $\mathrm{CAD}$ systems. This integration allowed two of the CAD stations to be installed in the DIII-D building.

\subsubsection{PLAN FOR COMPUTER CONNECTION TO RUSSIA}

A network meeting was convened by GA on June 10 to discuss the feasibility of establishing a realtime computer network connection to Russia. There were 27 people in attendance from many Fusion and High Energy Physics sites, as well as representatives from ESnet, NASA Sciences Internet, National Science Foundation network, DOE, SDSC, CERFnet, and visitors from the Confederation of Independent States (CIS). Considerable information was exchanged. There is a very strong desire across the scientific community to establish realtime computer connectivity with the CIS. This meeting was the impetus for a white paper written by the ESCC and presented to the DOE seeking officially sanctioned connectivity. 



\section{PROGRAM DEVELOPMENT}

\subsection{PROGRAM DEVELOPMENT OVERVIEW}

During FY92, an extensive effort was devoted to developing a new DIII-D Tokamak Long Range Plan for the next seven years, during which time DIII-D will be the largest operating U.S. tokamak in the post-tokamak fusion test reactor (TFTR) era. This plan was developed in collaboration between General Atomics (GA), Lawrence Livermore National Laboratory (LLNL), Oak Ridge National Laboratory (ORNL), and University of California at Los Angeles (UCLA) with numerous inputs from Sandia and other laboratories and universities. Advice and recommendations were incorporated from a National Workshop held in September with input from over a hundred participants, half of whom were collaborators from laboratories and universities other than $\mathrm{GA}$.

The long-range DIII-D program plan is aimed at the long-term objective of developing the foundation for an attractive DEMO reactor and in the near term supports the International Thermonuclear Experimental Reactor (ITER), tokamak physics experiment (TPX), Transport Task Force (TTF) and the development of an advanced tokamak concept. To carry out this program requires upgrades of DIII-D tokamak subsystems to perform divertor development and advanced tokamak experiments. DIII-D is the only tokamak in the world proposing to investigate these two elements in a coherent integrated program. The major elements of this program are schematically illustrated in Fig. 6.1-1 and the needed upgrades in facility capabilities is shown in Fig. 6.1-2.

There were two main DIII-D program development activities in FY92. These were the Advanced Divertor Program (ADP) and the $110 \mathrm{GHz}$ Electron Cyclotron Heating $(\mathrm{ECH})$ and Current Drive program. These programs are targeted at providing new capabilities which will facilitate DIII-D experimental programs oriented toward the development of steady-state current drive and boundary layer control technologies called for in the DIII-D Long Range Plan. Since these areas are recognized as important points 


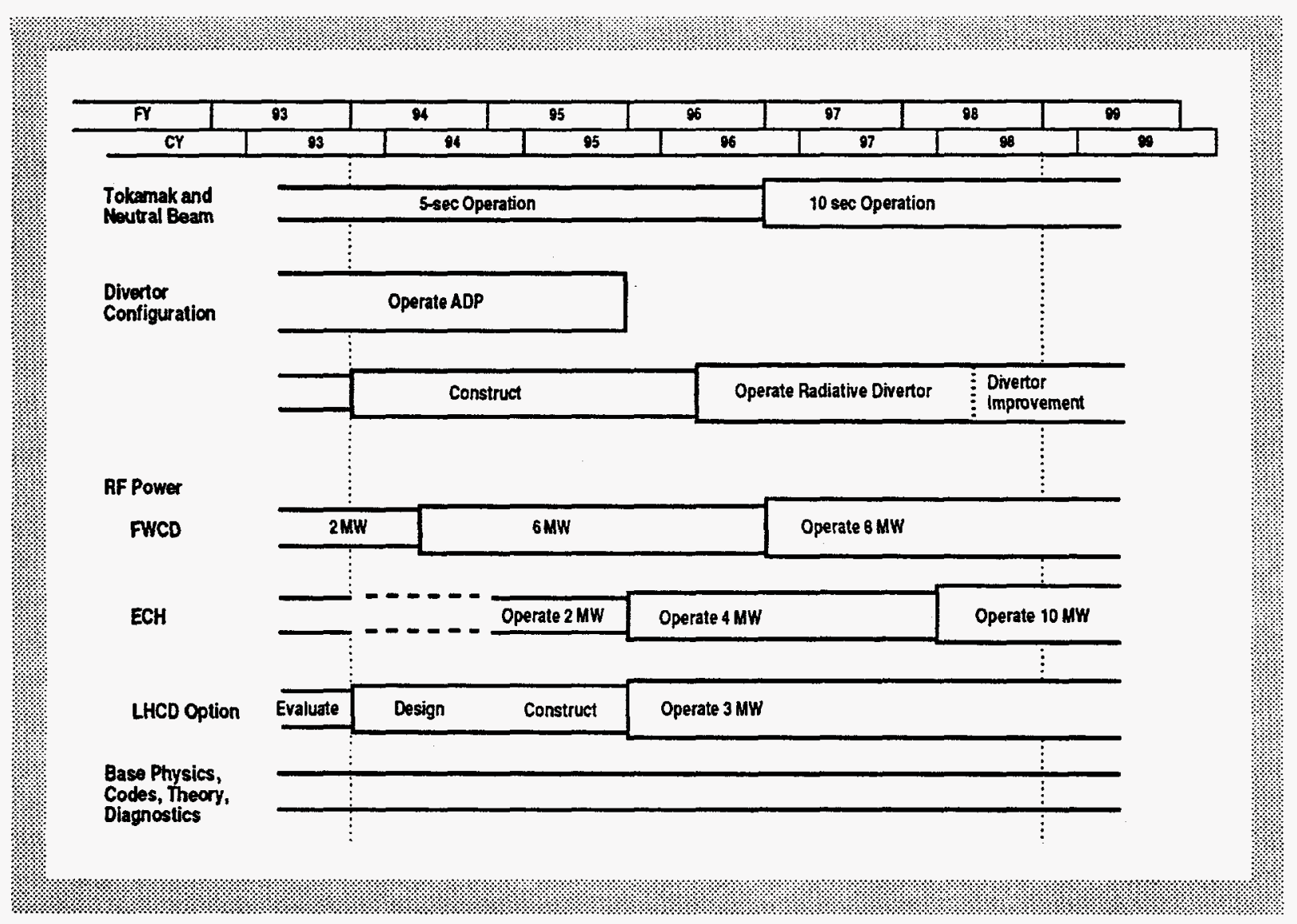

Fig. 6.1-1. Dill-D facility capabilities and upgrades.

in the development of ITER and TPX designs, we expect that results from these DIII-D activities will be of particular interest during the next several years.

\subsection{ADVANCED DIVERTOR PROGRAM}

\subsubsection{OVERVIEW}

The implementation of hardware supporting the ADP provides a unique opportunity to carry out DIII-D experiments aimed at developing new boundary layer control techniques. Results from these experiments are expected to open up improved performance parameter regimes and lead to an overall reduction in critical component failure risks. The principal experimental attributes associated with the ADP are: (1) provide density control during the high confinement $\mathrm{H}$-mode phase of the discharge for transport and current drive studies, (2) produce low collisionality plasmas for current drive experiments, (3) break the linkage which is typically 


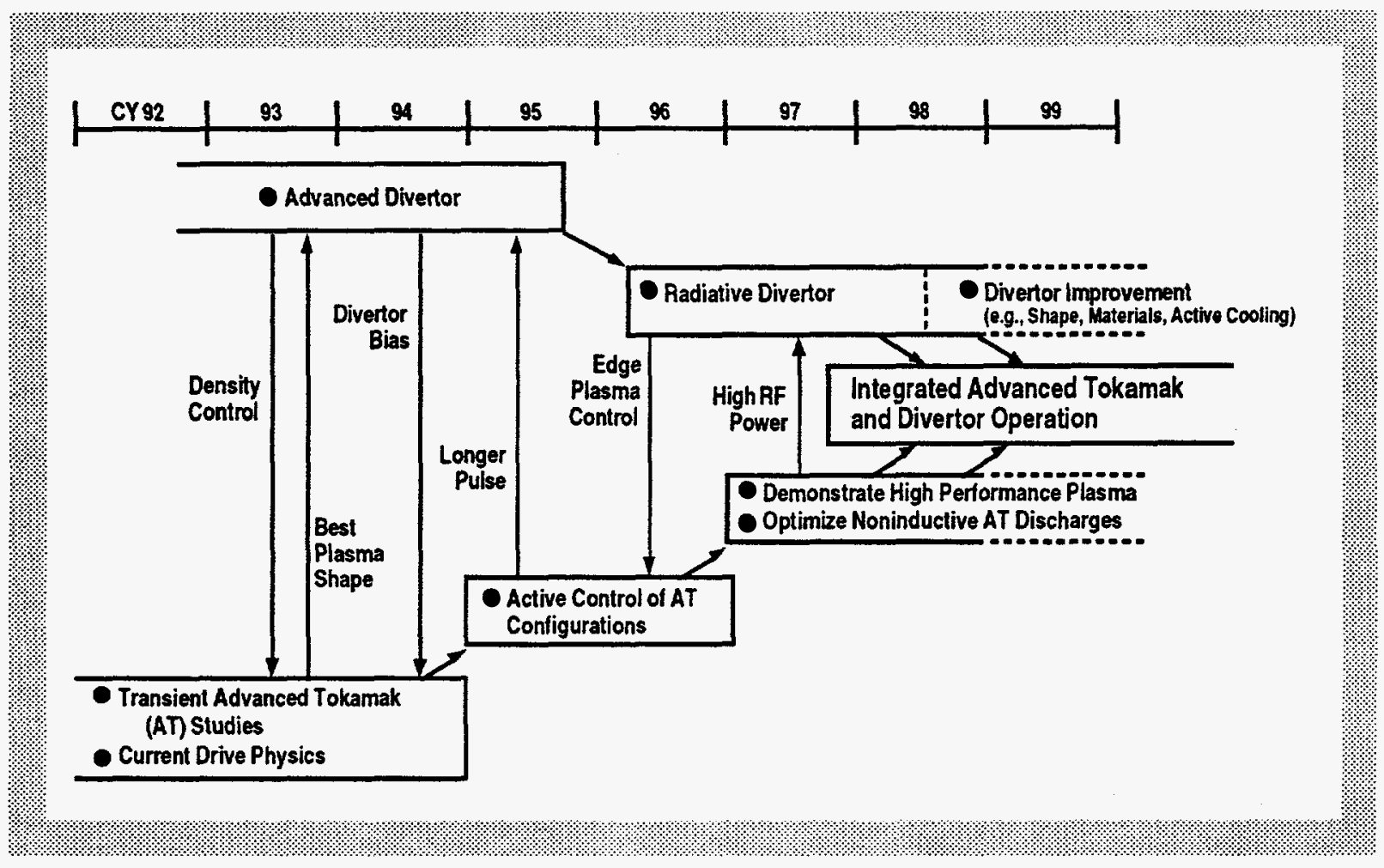

Fig. 6.1-2. The DIII-D long range plan is a coherent integrated program.

found to exist between the plasma current and the electron density, (4) provide a means of injecting helicity into the discharge for steady-state current drive, (5) to control and minimize energy deposition profiles on the divertor target plates by sweeping the bias ring voltage, and (6) study the effects of edge currents on the plasma stability.

The ADP work in FY92 began by completely rebuilding the electrical insulation of the ADP ring electrode and its electrical and cooling water leads to a new design, in order to overcome damaging electrical arcs that plagued the original design. In the new design, the lead geometry was simplified, and only hard ceramic insulators with long electrical paths were used. The new insulation performed well and vastly improved the reliability of the electrode system, and noninductive tokamak startup experiments were performed with up to $750 \mathrm{~V}$ applied to the electrode.

Due to limited DIII-D run time in FY92, only four experimental days were dedicated to ADP experiments. Considerable attention was given to the response of the scrape-off layer (SOL) to divertor bias. Biasing was observed to affect both the SOL density and the impurity influx. The two effects are believed to be linked, and thus divertor bias offers a way to control 
SOL impurity shielding. First, divertor bias increased or decreased the SOL density, depending on the sign of $\mathrm{E} \times \mathrm{B}_{\mathrm{T}}$. The bias-induced SOL density increase was as much as twofold. Second, increased SOL density was associated with reduced impurity influx and vice versa, for all $\mathrm{B}_{\mathrm{T}}$ and bias sign combinations. This behavior is consistent with unbiased plasmas, where thicker and denser SOLs also afford greater shielding against entering impurities.

Furthermore, a preliminary measurement of the plasma potential in the SOL during divertor bias was made with the fast-insertion Langmuir probe. Although the interpretation of the probe data is sensitive to the secondary emission model employed, it appears that the midplane SOL plasma potential decreased by a few tens of volts during the application of $-140 \mathrm{~V}$ to the divertor electrode, relative to an unbiased case. If true, the data then indicate that most of the applied potential drops across a cathode sheath.

A "bias-sustained guard plasma" concept was proposed, and a preliminary experiment was attempted. In this concept, Ohmic heating of an outer layer of the SOL by electrode-driven current would sustain the guard plasma layer. The experiment achieved small modifications to the selected SOL layer. However, other data have shown formation of bias-sustained-like plasmas in the divertor private flux region. Recent analysis has clarified the conditions needed to obtain strong SOL Ohmic heating, and this experiment will be repeated.

A tokamak plasma was formed and sustained noninductively by the combined action of the divertor electrode and ECH. The plasma was formed in a strong toroidal and weak vertical magnetic field by ECH while the electrode drove electric current along the magnetic lines. As a result of the tightly helical magnetic lines, up to $10 \mathrm{kA}$ circulated toroidally for $100 \mathrm{~A}$ leaving the electrode poloidally. When the toroidal current was sufficiently large, the plasma bulged outward and reconnected into a torus, which then remained in a steady state as long as power was maintained. The steady-state toroidal current appears to have been driven by electron cyclotron current drive, but the torus formation can be interpreted as magnetic helicity injection. Ideas are being developed to extend this technique to higher levels.

\subsubsection{THERMAL ANALYSIS OF THE DIVERTOR CRYOPUMP}

The helium panel of the cryopump has been designed to minimize the radiation and conduction heat loads on the helium panel. The radiation heat load on the helium panel was determined by using FACET code to compute the view factors between cryopump surfaces and vessel walls, followed by finite element calculation by using the code TOPAZ2D. As a result of 
this analysis, a design with a very small radiation heat load on the helium panel has been achieved. The conduction heat load was minimized by providing a long conduction path between nitrogen and helium panel. The steady-state heat load on the helium panel due to conduction and radiation is estimated to be less than $10 \mathrm{~W}$.

In addition to the steady-state heat load, the helium panel will be subjected to resistive heating during plasma operation. This heat load is equivalent to an average $46 \mathrm{~W}$ over $13 \mathrm{sec}$. An additional particle heat load of $10 \mathrm{~W}$ is expected. Thus, during plasma operation, the helium panel will be subjected a heat load of $66 \mathrm{~W}$. Analysis shows that a flow rate of $10 \mathrm{~g} / \mathrm{sec}$ of liquid helium will limit the temperature rise to less than $1 \mathrm{~K}$ under these conditions. However, experiments showed that the flow to the helium panel is reduced during the transient if the panel is subjected to the expected heat load pulse. This happens because the production of helium gas in the panel increases the pressure in the panel and reduces the flow into the panel. This problem can be remedied by increasing the flow velocity of the helium through the panel and by reducing the resistance to flow out of the panel. This was confirmed by experiments. Hence, the helium panel geometry was modified to an annular geometry. Also, a provision has been made to include a Dewar in the outlet circuit of the helium panel to relieve the pressure increase inside helium panel. The present design is now capable of a heat load of more than $100 \mathrm{~W}$ for more than $10 \mathrm{sec}$ with very small surface temperature rise.

Another matter of concern is the possible temperature gradients in the cryopump components during $400^{\circ} \mathrm{C}$ bakeout of the DIII-D vacuum vessel. A computer program was developed to analyze this. The components are subjected to radiation and conduction heat transfer from vessel surface and in between each other. In addition, a resistive heating occurs because of induced currents. As a result of this analysis, it was concluded that the temperature gradients were within acceptable limits.

The ADP 50,000 $\mathrm{v} / \mathrm{sec}$ divertor cryopump design was completed. A full-scale liquid helium test loop was used to study the two-phase cryogenic fluid and thermal dynamics. Stagnated He gas bubble problems were identified and overcome, and the final design exceeds the original specifications. The divertor pump will be installed in early FY93.

\subsubsection{ADP Structural Analysis}

The decision to design a toroidally continuous cryopump for the DIII-D advanced divertor greatly reduced the risk of electrical breakdown during plasma disruptions. 
However, this required that the mechanical design of the cryopump be structurally designed to withstand the inductive generation of toroidal currents in the pump. The mechanical loads caused by these currents were the primary challenge in the design of the cryopump.

The loads on the pump were extrapolated from magnetic measurements from actual DIII-D plasma disruptions and scaled for a worst-case 3 MA disruption. Since the magnetic measurements may not encompass the disruption loads which will produce the maximum structural response, a slow-pulse and fast-pulse load time history was developed based on the conservation of plasma momentum. The slow pulse was obtained from the fast pulse measurements by doubling the time duration of the load and halving the load amplitude. The set of slow pulse loads produced larger displacements since the resonant period of the pump is close to that of the slow pulse time length.

Extensive dynamic analyses were performed to design supports for the cryopump to preclude impact between components and high stresses following disruption. Initial sizing of the vertical spring rates for the supports between the helium tube and the nitrogen shield and the support to the vessel wall was performed using spring-mass models. These models simulated the dynamic behavior of the helium tube, nitrogen shield, and the coolant feed lines. A sensitivity study was performed to determine that a manufacturing variation of $20 \%$ of the optimum stiffness values could be allowed without violating the design criteria for displacements and stress. Testing of the manufactured supports verified that the actual spring rates for the supports were within $6 \%$ of the optimum design values.

Stress analyses of the cryopump due to dynamic and thermal loads and buckling analysis of the cryopump were performed using a detailed finite element model of a $360^{\circ}$ model input to the COSMOS code. Dynamic load factors computed from this model were then used to perform detailed stress analysis of a $15^{\circ}$ sector of the pump. A fatigue stress analysis of the welded connections was performed to estimate fatigue life of the pump and is summarized in Table 6.2-1. The buckling safety factors of the major cryopump components is also presented in Table 6.2-1.

\section{3. $110 \mathrm{GHz}$ ECH SYSTEM}

The DIII-D radio frequency ( $\mathrm{rf}$ ) heating program is targeted to address specific needs not being considered on other devices and fosters DIII-D programmatic goals in the areas of 
confinement improvement, high beta operation, and long-pulse operation. A unique application of the DIII-D ECH system is to raise the temperature and beta of the electron component so that the damping of the traveling fast wave is strengthened. It is expected that $10 \mathrm{MW}$ of ECH $110 \mathrm{GHz}$ with $8 \mathrm{MW}$ of fast wave current drive at $120 \mathrm{MHz} 2 \mathrm{MA}$ of noninductive current at 5\% volume-averaged beta in DIII-D. The first phase of this program is to develop and operate a system with $2 \mathrm{MW}$ at $110 \mathrm{GHz}$ for up to $10 \mathrm{sec}$. The ECH system consists of four $500 \mathrm{~kW}$ gyrotrons to generate $110 \mathrm{GHz}$ microwave power with pulse widths up to $10 \mathrm{sec}$. Low-loss transmission systems transmit this power to the DIII-D tokamak.

TABLE 6.2-1

Cryopump fatigue Life and Buckling Loads

FOR 3 MA "SLOW" DISRUPTIONS

\begin{tabular}{lllll}
\hline $\begin{array}{c}\text { Cryopump } \\
\text { Component }\end{array}$ & $\begin{array}{c}\text { Maximum } \\
\text { Alternating Stress } \\
(\mathbf{M P a})\end{array}$ & $\begin{array}{c}\text { Fatigue Life } \\
\text { at R.T. } \\
\text { (Cycles) }\end{array}$ & $\begin{array}{c}\text { Buckling } \\
\text { Load } \\
\text { (N/m) }\end{array}$ & $\begin{array}{c}\text { Buckling } \\
\text { Safety } \\
\text { Factor }\end{array}$ \\
\hline He line & 175 (weld at feed) & $>10^{6}$ & 12,260 & 40 \\
Outer $\mathrm{N}_{2}$ shield & 127 (at weld) & $>10^{6}$ & 245,180 & 34 \\
Inner $\mathrm{N}_{2}$ shield & 101 (at weld) & $>10^{6}$ & 90,018 & 25 \\
$\mathrm{~N}_{2}$ shield plug weld & 455 & $4.5 \times 10^{4}$ & - & - \\
He feedline weld & 346 & $3.5 \times 10^{5}$ & - & - \\
Pump support & 673 (Inconel 718) & $5.0 \times 10^{5}$ & - & - \\
\hline
\end{tabular}

In late FY91, a gyrotron undergoing long pulse testing at Varian failed when the pulse length was extended to $2 \mathrm{sec}$ at $300 \mathrm{~kW}$. An in-depth review of the damage led to the concern that the design of the $110 \mathrm{GHz}$ gyrotron was marginal in several components (e.g., collector heat dissipation, the output mode sensitivity, and the beam tunnel and cavity thermal capacity). Work on the production gyrotrons was put on hold and the Development and Technology (D\&T) program took over responsibility to solve the technical issues.

Since the prospect of having usable gyrotrons in FY92 was low, the project redirected its efforts to validate the design of the waveguide components and to investigate the mode purity of the gyrotron output. In testing the mode converter and down tapers, the effective conversion efficiency of the mode converter was demonstrated to to be at the $86 \%$ level with $350 \mathrm{~kW}$ output and a $405 \mathrm{~kW}$ input assumed to be in the current mode. It was also demonstrated that the presence of the down taper had a major effect on the power transmitted. When the down taper is 
measured separately (mounted between the gyrotron and the dummy load), a 10\% drop in throughput power was observed; when the taper was further reduced to $1.6 \mathrm{in}$., the throughput power dropped by $25 \%$ (almost identical to that measured at the University of Kyoto). This behavior indicates the output power may have a large fraction in undesired modes, but the cause is unknown.

In February 1992, the $110 \mathrm{GHz}$ ECH power was used for the first time to heat a plasma. The system was configured to provide a pulse train of $\mathrm{ECH}$ power with a duty cycle of $5 \mathrm{msec}$ "ON" and $10 \mathrm{msec}$ "OFF." Two such pulse trains were launched into a plasma with a density of $1 \times 10^{13} \mathrm{~cm}^{-3}$ and a toroidal field of $2 \mathrm{~T}$. One pulse train had nine pulses and the second had twelve pulses (see Fig. 6.3-1). In both shots, the effect of the pulses was clearly observed on several plasma diagnostics, and it is estimated that the temperature of the electrons was

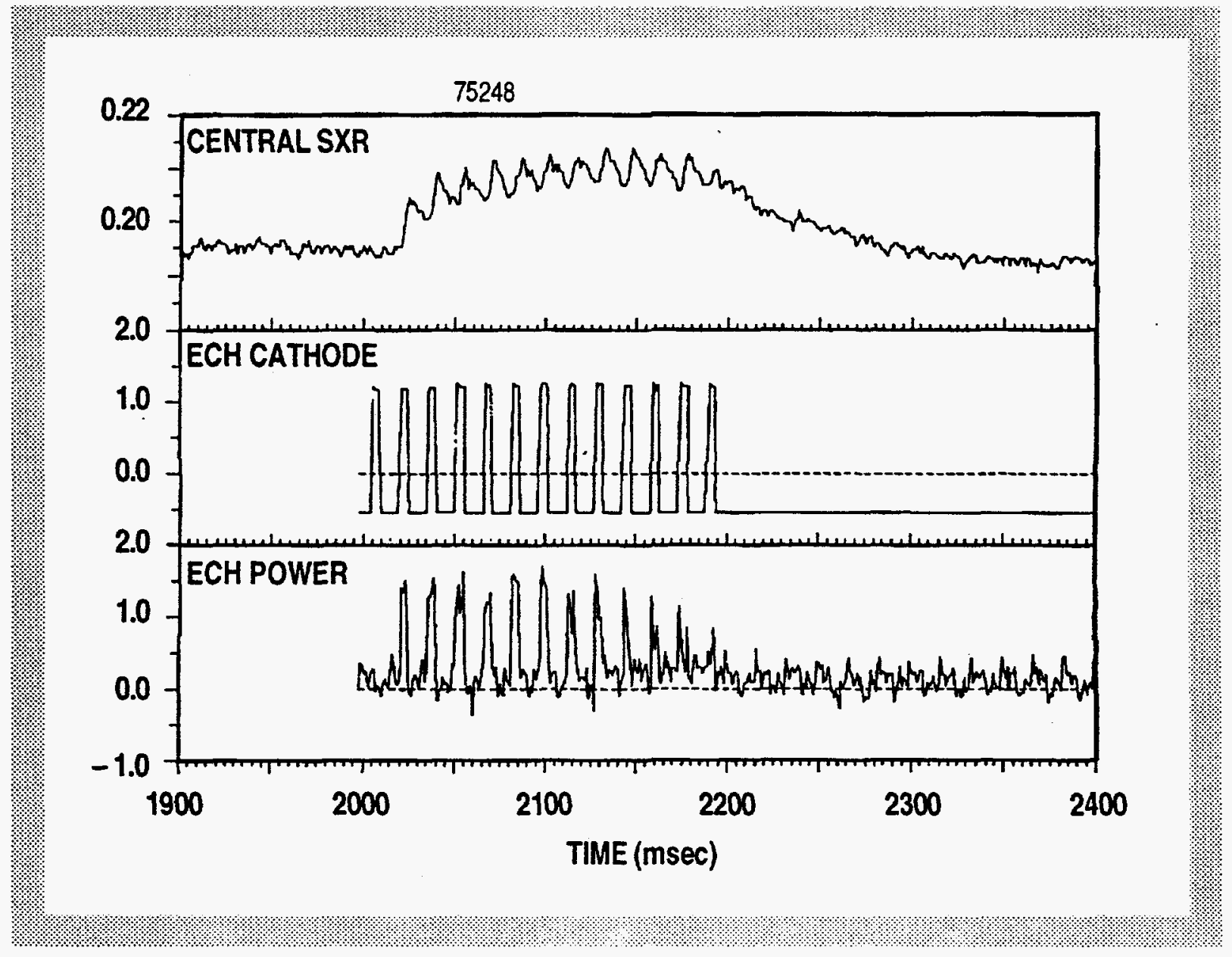

Fig. 6.3-1. Initial $110 \mathrm{GHz}$ heating on DIII-D. 
raised $100 \mathrm{eV}$ during the pulse duration. This value is consistent with the expectations considering the power levels ( $100 \mathrm{~kW}$ average) and density achieved.

The controls and tanks for System Nos. 2, 3, and 4 were completed and installed; the waveguide components for these three systems were also completed but were not installed. Work on water cooling the mode converters was stopped pending the successful testing of the redesigned gyrotron. The development of a water-cooled mode converter was also deemed questionable in light of new results from the University of Wisconsin which reported a Vaslov converter design with an efficiency greater than $90 \%$. This design would be much easier to cool. The University of Wisconsin will make its mode converter available for high power testing in FY93. 

SECTION 7

SUPPORT SERVICES 



\section{SUPPORT SERVICES}

\subsection{QUALITY ASSURANCE}

Fusion Quality Assurance (QA) engineers, inspectors, and support personnel maintained a high level of activity during 1992. Significant projects were the $110 \mathrm{GHz}$ Electron Cyclotron Heating (ECH) Systems, the Advanced Divertor Program (ADP), the DIvertor Material Exposure System (DIMES), and the DIII-D Graphite Tile Upgrade project.

\subsubsection{DESIGN SUPPORT}

1. Performed a physical layout in support of design engineering for the $110 \mathrm{GHz}$ waveguide run at the DIII-D $255^{\circ}$ azimuthal location. A compact HeNe laser was utilized in lieu of optical instruments due to space constraints.

2. Measured linear accuracy of the waveguide machining device stepping motors utilizing optical tooling instruments and provided data to DIII-D engineering.

3. Performed measurements of a neutral beam adjustable base support and provided data to engineering. This task was required as a result of a rod end failure.

4. Measured orientation of three DIII-D port flanges for the Fusion Diagnostics Group.

5. Reviewed and approved all DIII-D design drawings, specifications, procedures, and purchase requests. Participated in design reviews and chaired the Material Review Board (MRB).

\subsubsection{INSPECTION SUPPORT}

1. The majority of Quality Control $(\mathrm{QC})$ activities during the year involved receiving and source inspections of purchased and fabricated parts, subassemblies, and assemblies. Inspection activity was particularly heavy for the ADP, the $110 \mathrm{GHz}$ ECH System, the DIMES Project, and the Graphite Tile Upgrade Project. 
2. QA witnessed hydrotests of the Ion Cyclotron RF Heating (ICRH) pressurized waveguide tee to assure safe operation of the system. QA also witnessed hydrotests of an ECH tee and gas break and pneumatic tests on high-pressure He lines. At the request of the DIII-D Safety Committee, QC inspectors completed a comprehensive survey of potential hazards to operating and maintenance personnel. Thirty-six hazards were identified and corrected. QC also performed hardness tests; performed vacuum leak investigations; witnessed crane and lifting fixture proof-testing; monitored removal and replacement of neutral beam components; witnessed hydrotests of FRP 12 and 16 in. ECH water cooling lines; verified machine setups prior to machining operations; performed internal inspections of waveguides using bore scopes; and performed dimensional, fitup, alignment, and weld inspections on the $180^{\circ} \mathrm{R}-0$ bellows assembly installation.

Quality Assurance Representatives (QAR) completed over three weeks of support at the supplier's facility on the DIMES double-acting telescopic cylinder. In conjunction with DIII-D engineers, the General Atomics (GA) QAR assured that the supplier completed the cylinder in accordance with design requirements. GA's team presence prevented this complex system from being scrapped.

\subsubsection{AS-BUILT MEASUREMENT SUPPORT}

1. Measured and documented alignment variations of the neutral beam adjustable base support rod end.

2. Documented as-built locations of the recently installed cryostat for input into the CAD database.

3. Performed numerous dimensional inspections of the neutral beam ion source before removal and after reinstallation.

4. Documented as-built flatness variations of a neutral beam source bucket assembly.

5. Performed in-process inspections of the prototype advanced divertor outer shell to document the change in curvature after the internal cryogenic transfer line was welded.

6. Measured and documented ball raceway depth and stop screw height on four source isolation valves undergoing renovation. 


\subsubsection{OPTICAL TOOLING/AYOUT/ALIGNMENT SUPPORT}

1. Developed special tooling for the DIMES drive tube offset measurement task.

2. Completed a layout of the $110 \mathrm{GHz} \mathrm{ECH}$ waveguide run at the DIII-D $255^{\circ}$ azimuthal location.

3. Performed the semiannual building subsidence survey using optical instruments.

4. Checked alignment of the waveguide machining fixture rail guides-to-waveguide restraint supports utilizing optical tooling instruments.

5. Performed many internal bore scope inspections of $110 \mathrm{GHz}$ corrugated waveguides.

6. Verified linear accuracy of the waveguide stepping motors using optical tooling instruments.

7. Completed elevation measurements of the translating roof rails and girders in the high bay area to determine the amount of subsidence.

8. Optically aligned the $150^{\circ}$ neutral beam adjustable base front flange and bellows to the beam centerline before welding the assembly.

\subsubsection{QA SYSTEM IMPROVEMENTS}

1. Participated in a Fusion Group effort to improve engineering procedures. This effort is continuing.

2. Participated in initial efforts to adopt Total Quality Management (TQM) principles in the Fusion Group. Initial training has concentrated on continuous improvement procedures.

3. Fusion QA has been accepted as an authorized user of the Safety Performance Measuring System (SPMS)/Occurrence Reporting and Processing System (ORPS) which will allow visibility of equipment and system problems experienced by the Fusion community. 
4. Fusion QA now inputs inspection and acceptance information into the Dun \& Bradstreet Millennium Accounts Payable/Purchase Order System. In addition to providing a database of inspection activities, the system requires QA acceptance of articles to be inspected before the supplier is paid, which is expected to decrease the number of submitted parts not meeting design requirements.

\subsubsection{OTHER SUPPORT}

1. Conducted the quarterly visual inspections of all DIII-D hoisting and rigging equipment.

2. Monitored numerous high consequence lifts.

\subsection{PLANNING AND CONTROL}

The Planning and Control Group supported operation and maintenance of the DIII-D facility. Planning and control provided long-term program planning, as well as day-to-day scheduling (cost control, preparation of Field Task Proposals and Cost and Fee Proposals), processing of purchase requests, expediting and reporting of status. These support activities are essential to the performance of the program within prescribed budgets and schedules. These planning activities (budget, schedule, resource) enabled us to maximize the utilization of available resources for accomplishment of program goals.

Major planning activities during FY92 included the Advanced Divertor, vessel outer wall tiles, diagnostic upgrades, machine operations and maintenance, and a major five-month vent (beginning in August).

\subsection{ENVIRONMENT SAFETY AND HEALTH}

\subsubsection{FUSION AND DIII-D SAFETY}

The Fusion safety program provides for the safe operation of the DIII-D facility and for a safe working environment for employees and visitors. Special programs address high voltage and high current, high vacuum systems, ionizing radiation, microwave radiation, cryogenics and the use of power equipment and machine tools. The "DIII-D Safety Procedures for Facility and Equipment Operation" contains policies and procedures that 
specify safety rules and procedures that must be adhered to while working at the DIII-D site. Fusion works closely with GA's Licensing, Safety and Nuclear Compliance organization in areas such as health physics, industrial hygiene, environmental permitting and industrial safety.

Fusion has established a Safety Committee in accordance with company policy as a means of focusing on and addressing both the numerous safety issues faced daily and longer range safety needs and goals. The Fusion Safety Committee is comprised of Fusion representatives from various Departments within the Fusion group, including top management, supervisors and technicians. The safety Committee chairman is the DIII-D Program Director and the assistant chairman is the Fusion Safety Officer. The Safety Committee meets twice a month to address safety activities and concerns of the Fusion group such as: hazardous work requests, radiation work authorizations, accident/incident reports, near misses, equipment malfunctions, accident avoidance programs, supervisor involvement, training, inspections, access control procedures and high voltage hazards. The Safety Committee also solicits specialized help from any one of the five Fusion Safety Subcommittees during reviews of lasers, electrical systems, vacuum systems, the use of cryogenic or the use of chemicals.

In addition to the Fusion Safety Committees oversight of activities at DIII-D, two individuals are dedicated full-time for onsite "preventive" safety involvement. Their activities include writing and reviewing procedures, developing and conducting special training classes, conducting inspections and follow-up, interfacing and coordinating with GA's Licensing, Safety and Nuclear Compliance organization and providing continuous oversight to assure compliance with established safety policies and procedures.

The DIII-D Emergency Response Team consists of individuals involved directly with maintenance and operation of the DIII-D equipment. They are trained in CPR, first aid, SCBA and the use of fire extinguishers, evacuation and crowd control and facility familiarization. The team can respond within seconds to provide immediate assistance until the company Emergency Medical Technicians (EMTs) arrive.

Safety Inspections are conducted throughout the year to promote an active Hazard Prevention Program. The inspections are conducted by a combination of Fusion, GA Licensing Safety and Nuclear Compliance personnel and outside consultants. 
A report is provided to the Fusion Safety Committee where corrective action assignments are made. DIII-D inspections include:

Inspection

Site Inspection (GA Personnel)

Electrical Consultant

CAL/OSHA Consulting Service

Insurance Carrier Inspection

S.D. City Fire Department CEDMAT

GA Safety Committee Hazards Survey

DOE-SF Safety Review Inspection

Frequency
Monthly
Yearly
Yearly
Yearly (Multiple)
Yearly
Yearly
Semi-Annual

The Fusion Safety Officer is responsible for tracking the progress of all discrepancies and ensuring resolution.

All new employees and collaborators must go through a thorough and comprehensive safety indoctrination by the Fusion Safety Officer and Pit Coordinator. They are informed of the specific potential hazards that are present daily at DIII-D and the special safety precautions and rules that apply, with specific emphasis on the areas where they will be working. Subcontractors also receive a similar indoctrination.

\subsubsection{FY92 SAFETY}

\subsubsection{Training}

Training is all-important to the safety of both personnel and equipment. Due to the complexity of the DIII-D site and its operation, numerous safety training classes are conducted. Subjects of the classes include, but are not limited to: confined space entry, back injury prevention, radiological safety, laser salety, hazard communication, cryogenic safety, crane and forklift operation, lockout/tagout, the national electrical code, machine shop tool usage and basic industrial safety requirements.

The Fusion Safety Officer attended a week-long OSHA Training Institute Workshop covering 29 CFR 1910 "Welding Regulations" and the National Safety Council Annual Conference on Safety and Health and attended two seminars "Beyond Behavior Modification: What's New in Safety Motivation", and "The Psychology of Accident Prevention." 
The yearly DIII-D Emergency Response Team training sessions were presented by the GA Emergency Services Department in a joint training effort with the Fusion Safety Department. They include the following: Facility Familiarization Training; Fire Extinguisher Training; Hydrant Hook-Up Training; SCBA Training; CPR Training.

A fire and emergency evacuation drill was conducted by GA Emergency Services Department which exercised the DIII-D Emergency Response Team as well as the emergency preparedness of all DIII-D personnel.

A vessel extrication training session was conducted where the DIII-D Emergency Response Team and Emergency Services personnel practiced removing a simulated person from inside the DIII-D vessel. Procedures and emergency equipment were modified due to lessons learned during this training session.

A new "Working Safely and Effectively in the DIII-D Pit" training class was presented to the Fusion employees who work in the DIII-D pit. This class is now mandatory for all employees who will be working in the DIII-D pit with retraining required once a year.

Numerous Confined Space Entry Classes were provided prior to and during the vent to enable the safe entry into the DIII-D vessel confined space. Every individual who enters any confined space must have attended this one-hour training class. At the close of FY92, we had 125 confined space trained individuals.

A six-hour radiation training course was offered to all new DIII-D employees and Job Shoppers to fill the Health Physics/Nuclear Regulatory Commission (NRC) radiation training requirements. Three radiation refresher courses were also given by the Fusion Safety Officer to all Fusion employees who have previously had the complete course and only needed the yearly update.

Hazard Communication Training was offered by GA's Industrial Hygiene Department covering Material Safety Data Sheets, container labeling and hazard identification.

There were two "Backs-to-School" classes presented for DIII-D employees by a private consultant. These three hour classes emphasized how to care for your back and avoid injury and included both classroom studies and an active participation exercise session. 
The Fusion Safety Office has started the design of a new Stationary Power Tool training program. The program would require that each individual have the proper training on the safe use of each stationary power tool prior to operation.

During FY92, 40 new employees and long-term visitors received a safety indoctrination from the Fusion Safety Officer.

\subsubsection{Inspections}

As stated above, a number of DIII-D inspections occurred during the year. In addition to these, the following inspections were conducted. A DOE Multidiscipline Safety Review follow-up audit was conducted by representatives from DOE/SF. The overall comments from the representatives were positive. They stated how impressed they were with our safety program and the importance we placed on resolving the action items that were noted from the previous inspection. They appreciated our pro-active approach to safety concerns.

A complete gas bottle inspection was conducted by our gas bottle vendor and a representative from Facilities Engineering. Out of 160 bottles inspected, none were unchained or improperly stored. The vendor was impressed with our high-pressure gas bottle safety program.

A representative from American Nuclear Insurers toured the DIII-D facility to inspect and check for unusual fire hazards and other areas that might put the facility at risk. His emphasis was the disposition of surplus equipment and materials. A chemical inspection and inventory was conducted of the entire DIII-D facility, cataloging all hazardous substances. Missing Material Safety Data Sheets (MSDSs) were requested and have now been cataloged for employee access in the Safety Library and the Fusion Safety Office. These data sheets have also been cataloged in the computer database system for easy reference and back up.

\subsubsection{Other Activities}

The Fusion Safety Officer participated in the 14th Annual Symposium on Fusion Engineering (hosted in San Diego) as a co-chairman of the Environment, Safety and Health session.

As a result of an accident involving a Japanese scientist at Lawrence Livermore National Laboratory (LLNL) in February 1992, the U.S. DOE and Japan formed a Joint Working Group (JWG) to address the safety issues in interinstitutional collaborations. Representatives 
GA-A21186; FY92 DIII-D Research Operations Annual Report

in both Japan and the United States realized that safety issues needed to be examined more closely in order to increase the level of safety assurance. The JWG consists of five representatives from the U.S. and five from Japan. The Fusion Safety Officer was selected to represent both GA and the U.S. industrial contractors. This group is supported by DOE and is led by Steve Rossi of the Office of Fusion Energy. The first meeting was held October 23, 1992 in Germantown, Maryland.

The Fusion Safety Officer also visited the University of Wisconsin for two days in an interlaboratory safety assessment/collaboration visit.

A Chemical Safety Subcommittee was established to assist the Fusion Safety Committee. Their charter is to discuss the chemical hazards and safety procedures to be included in applicable Hazardous Work Authorizations (HWAs) and to advise the Fusion Safety Committee of potential chemical safety concerns. This subcommittee joins those already established for lasers, cryogenics, electrical systems and vacuum systems.

The DIII-D Safety Committee Membership was reviewed, brought up to date, and temporary rotating members were replaced by new members. This committee met a total of 25 times. Listed below are some of the tasks that were accomplished:

1. Reviewed and approved 35 HWAs after appropriate recommendations and changes by the Safety Committee and select Safety Subcommittees.

2. Reviewed eight minor first aid incidents which involved no lost time or property damage, five accidents that required minor medical treatment, and one lost time accident.

3. Reviewed the monthly inspection discrepancy reports of the DIIl-D site. These include a request from the Fusion Safety Officer for the CAL/OSHA Industrial Hygiene consultation services, an Electrical Consultant Inspection, a GA Hazard Survey Inspection, monthly Fusion Safety Committee inspections, daily walkaround inspections, and follow-up visits from the San Diego Fire Marshal's Hazardous Materials Inspection Team and the DOE Multidisciplinary Review Committee. In all, a total of 472 safety discrepancies were noted, and at the close of FY92, all of these discrepancies had been corrected. 


\subsubsection{RADIATION SAFETY}

The State of California, NRC, and DOE set criteria for the maximum radiation exposures for the general public and employees. [Refer to "Radiation Aspects of DIII-D," by Project Staff; J. Kim, J.L. Luxon (eds.), February 1989.] Section 30268 of the State of California regulations state: No user shall possess sources of radiation in such a manner as to create in any uncontrolled area, from such sources, radiation levels which could cause any individual to receive a dose to the whole body in excess of:

1. Two (2) millirem in any hour.

2. One hundred (100) millirem in any seven consecutive days.

3. Five hundred (500) millirem in any one year. (The State of California limit will decrease from 500 to 100 millirem per year in 1994.)

GA operates DIII-D according to the general requirement that the exposure of the public to radiation be kept as low as reasonably achievable (ALARA). In the spirit of this goal, GA and DOE now operate DIII-D at a guideline of $20 \mathrm{mRem} / \mathrm{yr}$, at the DIII-D site boundary, with a DOE administrative allocation of $5 \mathrm{mRem} /$ quarter. An important consideration is the radiation level experienced by members of the staff, either in carrying out device operations or in working in the facility, especially near or in the tokamak after operations in the presence of residual radiation due to activation of device materials. All GA DIII-D personnel, visitors, collaborators, and contractors who are in the DIII-D facility for more than $18 \mathrm{hr} / \mathrm{qtr}$ during operations are classified as radiation workers. They receive radiation training and carry radiation badges during operations. The present limiting criteria is that worker exposure be less than $1250 \mathrm{mRem} / \mathrm{qtr}$. The DIII-D program presently applies a limit of $300 \mathrm{mRem} / \mathrm{qtr}$ for individuals. The two most restrictive conditions for DIII-D are the exposure of individuals who work in the vessel for prolonged periods of time and the site accumulated radiation at the boundary limit.

Radiation levels for all proposed DIII-D experiments are estimated and approved in accordance with the ALARA principal before authorization is given to conduct any experiment. Due to the present equipment limitation on pulse length, the available heating power, and the budgetary limitation on operating weeks, the actual site boundary exposure has been only 3.7 to $5.5 \mathrm{mRem} / \mathrm{yr}$. 
GA-A21186; FY92 DIII-D Research Operations Annual Report

In DIII-D operation through 1992, the highest D-D neutron rate produced was $5.8 \times 10^{15}$ neutrons $/ \mathrm{sec}$, and the largest number of neutrons produced on a single shot was $5.7 \times 10^{15}$ neutrons. For the present DIII-D radiation shield, $1.5 \times 10^{17}$ neutrons is the equivalent of $1 \mathrm{mRem}$ at the site boundary. The highest site-boundary radiation rate produced is $0.037 \mathrm{mRem} / \mathrm{sec}$, and the maximum site radiation produced per shot was $0.038 \mathrm{mRem}$.

The neutron and gamma radiation produced at DIII-D is constantly monitored during operations at the site boundary with the level kept below $20 \mathrm{mRem}$ per year. Personnel dosimeters are worn by all individuals while on the DIII-D site and if entrance into the machine pit is required between shots, an alarming dosimeter is worn in addition to the TLD badge. Prior to unrestricted machine pit access, the pit is surveyed and the areas requiring monitoring before entry are cordoned off until radiation levels fall below the DIII-D procedural control limits. Workers in the vessel carry counters and each entry is controlled, monitored, and recorded.

\subsubsection{FY92 RADIATION SAFETY}

Radiation management tasks include monitoring the site boundary radiation, monitoring the dose exposures of individuals from pit runs and vessel entries, ensuring compliance with legal limits, DOE guidelines and DIII-D procedures, monitoring material for activation, maintenance and operation of the radiation monitoring detectors (neutron and gamma), and maintaining a database of radiation measurements and of personnel dose exposures.

The total neutron radiation at the site boundary for FY92 was 2.8 millirem, the total gamma radiation was 1.4 millirem, giving a total site dose for the year of 4.2 millirem. (This is below the SAN DOE annual guideline limit of 20 millirem and the California annual limit of 500 millirem.)

The total dose exposure personnel received was kept below the DIII-D procedural limits of 25 millirem per day, 100 millirem per week, and 300 millirem per quarter (1200 millirem per year). The highest dose accumulated by an individual from pit runs 
and vessel entries (but not operations) for FY92 was 365 millirem. A total of 129 individuals received such doses as follows:

\begin{tabular}{rcccc}
$\begin{array}{c}\text { Dose Range } \\
\text { (millirem) }\end{array}$ & Number of People & & $\begin{array}{c}\text { Dose Range } \\
\text { (millirem) }\end{array}$ & Number of People \\
\cline { 2 - 3 } $0-25$ & 104 & $201-250$ & 0 \\
$26-50$ & 11 & $251-300$ & 0 \\
$51-100$ & 9 & $301-350$ & 2 \\
$101-150$ & 2 & $351-400$ & 1 \\
$151-200$ & 0 & &
\end{tabular}

All doses were logged in the database of personnel radiation doses.

The periodic (every 2 year) survey of the site boundary radiation continued. There were no unexpected differences from past measurements.

The annual radiological safety audit of the DIII-D facility was conducted by the manager of GA Health Physics, 19 action items were identified and all but one were completed in a timely matter. The outstanding action item concerns renewal of a work authorization.

Two radiation safety training classes were held for new personnel.

An 11 inch square hole was made in the west pit wall for the ADP cryolines and the remaining voids filled with neutron shielding material. The site boundary radiation was unchanged by this modification within the error bars of the measurements (30\%); calculations had predicted a $2 \%$ increase. The neutron radiation at the nearby walkway (15 feet from the wall) was $20 \%$ higher but within the error bars of the measurements (25\%); calculations had predicted an $18 \%$ increase. The gamma radiation at the walkway increased by a factor of 3 but is only $10 \%$ of the neutron radiation.

Preliminary analysis of shielding requirements to obtain a factor of 10 additional shielding for use in future upgraded operations was made. The results indicated that an additional 8 inches of poly would be needed as opposed to the 4 inch answer arrived at by extrapolating the old 12 inch calculation. A detailed plan would also require structural analysis. 
The DIII-D work authorization (WA) was reviewed, the radiation procedures were modified slightly, the authorized users list was updated, and the completed WA was approved by GA Health Physics. One improvement incorporated in the new WA is the ability to set up a hot machine area in the Building 34 machine shop instead of being restricted to the diagnostics lab.

A barrel of waste trash and a barrel of contaminated oil were picked up by the waste yard.

The vessel was vented five times: for 50 days starting October 31 , for one day on January 16, for six days starting February 27, for 10 days starting June 29, and for the vent starting August 17 (45 days in the 4th quarter) which continues into next year. Radiation monitoring was performed for all vessel entries.

Many radiation monitoring activities were required for vent related tasks. Items removed from the pit were checked for activation and a large number of radiation work permits were written for work on activated items. A "hot" shop was set up in the diagnostics lab for machining of activated material and decontaminated afterwards. Activated items were also sent to the GA hot shop and to other vendors for modifications.

A large number of items removed from the machine pit have been checked for activation and those with radiation levels above background are in storage.

During the February vent entry four tritium wipe samples of the carbon tile surfaces were taken for the first time. The limiter read high $\left(140,000 \mathrm{DPM} / 100 \mathrm{~cm}^{2}\right)$ compared to the other surfaces (up to $2200 \mathrm{DPM} / 100 \mathrm{~cm}^{2}$ ). The higher level of tritium on the limiter was surprising but may be due to beam target interactions from impinging beam ions. A survey of tritium inside the vessel was performed during the July vent. A total of 24 wipe samples were taken from various locations; including wipes from the carbon tiles on the floor, ceiling, inner wall, outer wall, and limiters, and wipes of the Inconel outer wall. Results ranged from 100 to $6200 \mathrm{DPM} / 100 \mathrm{~cm}^{2}$. The highest concentrations of tritium were on the limiters followed by the carbon tiles on the outer wall. The Inconel tiles, Inconel outer wall and non-outer wall carbon tiles had lower concentrations of tritium.

The carbon tiles removed from the machine during the August vent for cleaning were sampled for activation, removable alpha, removable beta, and removable tritium. The 
samples were taken from the approximately 1600 tiles at a sampling rate of one sample for every 10 tiles. The tiles showed no radiation above background for the activation, removable alpha, and removable beta. The 173 tritium samples had contamination levels on 163 tiles of less than $500 \mathrm{DPM} / 100 \mathrm{~cm}^{2}$, and on 10 tiles the contamination levels ranged up to $2500 \mathrm{DPM} / 100 \mathrm{~cm}^{2}$. California regulations allows a release for unrestricted use for levels below $4000 \mathrm{DPM} / 100 \mathrm{~cm}^{2}$ but the DOE release limit is $1000 \mathrm{DPM} / 100 \mathrm{~cm}^{2}$; the DOE limit is followed.

Discussions and plans for the carbon tile cleaning procedure and required radiation monitoring were held. An estimate was made of the tritium which may be released in the grit blasting of the tiles. Based on a worst case assumption (that $40 \%$ of all the tritium generated in 1991 and 1992 was retained in the tiles) the tritium concentration level in the grit blaster output would be at the level which allows unrestricted access $\left(2 \times 10^{-13} \mathrm{Ci} / \mathrm{ml}\right)$ and would be below the level allowed for occupational exposure $\left(5 \times 10^{-12} \mathrm{Ci} / \mathrm{ml}\right)$. The release requirements for sending the tiles to an outside vendor for baking were discussed with GA Health Physics but a plan had not yet been finalized.

\subsection{VISITOR AND PUBLIC INFORMATION PROGRAM}

Tours of the DIII-D facilities are open to organizations and institutions interested in fusion development (colleges, schools, government agencies, manufacturers, and miscellaneous organizations). These tours are conducted on a noninterference basis and are arranged through the DIII-D tour coordinator whose responsibilities include security, arranging tour guides, and scheduling tours. During the year, 1629 people toured DIII-D to give a total of 9468 during the last eight years.

Special tours during the year included:

- Harvard Business School Club of San Diego

- Contractor: Snyder \& Langston

- UC Irvine Physics Students

- Monte Vista High School Physics Students

- Pacific Beach Presbyterian Mens Club

- Northrop Corporation Representatives

- Electric Car Club of San Diego
- Outreach Program

- La Jolla Education Association

- MODCOMP User Exchange Conference

- Director of Naval Research Laboratory

- ASME ANS Tour

- SDSU IEEE Student Club

- DIMES Workshop Participants 
- FBI Personnel

- Director of the U.S. Consul for Energy Research

- Association of American Geographers

- Torrey Pines High School Physics Class

- Santa Fe Christian High School Physics Class

- Cal Tech Physics Students

- Tierra Bonita Elementary School

- President of JAERI, Mr. Shimomura

- Santana High School Physics Class

- Point Loma Nazarene College Physics Class

- San Diego City Environmental Biology Class

- American Institute of Plant Engineers

- UCSD Nuclear Energy Class

- Cuyamaca College Engineering Class

- Association of Computerized Machinery
- Electric Power Research Institute (ERPI)

- Hoover High Calculus/Physics Class (LA)

- SDSU Geography Class

- ICF Workshop

- Chula Vista and Morse High School Science Students

- Bird Rock Elementary (5th Grade)

- Alcott Elementary (5th Grade)

- UCLA Engineering Students

- USD Physics Students

- Southwest and Montgomery High School Science Students

- Mira Costa College Physics Students

- Director of the NRC

- ECH Transmission Line Workshop

- UCLA/Japan on M/W Diagnostics

- Senator J. Bennett Johnston 

SECTION 8

\section{CONTRIBUTION TO ITER PHYSICS R\&D}





\section{CONTRIBUTION TO ITER PHYSICS R\&D}

The DIII-D Research Program has made substantial contributions to the Physics Research and Development (R\&D) requirements put forward by the International Thermonuclear Experimental Reactor (ITER) program. The most recent statement of these needs is made in the ITER Physics R\&D Needs for 1991, 1992, and Beyond. These needs are identified in terms of 51 topics in five major categories. The DIII-D program is contributing in 43 of these areas. These areas of contribution are summarized in Table 8-1.

There has been no formal report required in FY92 during the transition from the Conceptual Design Activity (CDA) to the Engineering Design Activity, but considerable progress has been made nonetheless. These contributions are summarized in Table 8-2.

Major contributions are being made in the areas of Power and Particle Exhaust Physics and Enhanced Confinement where the DIII-D program has been particularly productive. The radio frequency ( $\mathrm{rf}$ ) program is anticipated to provide results on fast wave current drive (FWCD) and electron cyclotron heating (ECH) and current drive (CD). The DIII-D program of disruption studies has been strengthened to provide a stronger database for ITER.

The DIII-D program has also contributed significantly to the organizational activities of the U.S. ITER Home Team. J. Luxon has served to coordinate the Physics R\&D Program, and Punit Gohil has coordinated these activities within the DIII-D program. Arnie Kellman has been Task Leader for Disruption Studies in the U.S. program. Robin Snider has been particularly active in diagnostic activities for ITER. 
TABLE 8-1

Planned Dill-D Contributions

TO ITER LONG-TERM PHYSICS R\&D

DIII-D

1. Power and Particle Exhaust Physics

1.1. SOL and Divertor Physics
a. Divertor Conditions
b. Geometry Variations
c. Hot Spots
d. CD Divertor Conditions
e. Impact of Fueling
f. Edge localized modes (ELMs)

1.2. Impurity Radiation and Transport

a. Radiating Plasma Edge

1.3. He and $H$ Exhaust

1.4. Divertor Control

1.5. Plasma-Facing Materials
a. Conditioning Methods
b. Conditioning Between Shots

1.6. Alternative Divertors

2. Disruption Control and Operational Limits

2.1. Disruption Characterization
a. Runaway Electrons
b. Soft Current Quench

2.2. VDES

2.3. Disruption Avoidance and Control
a. Disruption Pre-Cursors

2.4. Beta Limits
a. Profile Effects
b. Equilibrium Inductive Operation
c. Sawteeth Effects
d. Fast lon Effects

2.5. Density Limit 
TABle 8-1 (CONTINUed)

\begin{tabular}{lc}
\hline & DIII-D \\
\hline 3. Enhanced Confinement & \\
3.1. Steady-State Operation & $\checkmark$ \\
a. Energy Confinement Scaling & $\checkmark$ \\
b. Plasma Particle Transport & $\checkmark$ \\
c. Momentum Transport & $\checkmark$ \\
3.2. Control of magnetohydrodynamics (MHD) Activity & $\checkmark$ \\
3.3. Transport Mechanisms & $\checkmark$ \\
a. Plasma Turbulence & $\checkmark$ \\
Optimization of Operation Scenario and & \\
Long-Pulse Operations & \\
4.1. Long-Pulse Operation & $\checkmark$ \\
a. Bootstrap Current & $\checkmark$ \\
b. Lower Hybrid Physics & \\
c. FWCD & $\checkmark$ \\
d. Electron cyclotron current drive (ECCD) \\
e. Neutral beam current drive (NBCD) \\
f. Advanced CD \\
4.2. Optimization of Startup \\
a. LHCD Ramp-up \\
4.3. Plasma Shutdown \\
4.4. lon cyclotron RF heating (ICRH) \\
4.5. Pellet Ablation Models \\
a. Compact Toroids \\
Physics of a Buming Plasma \\
5.1. Single Particle Effects \\
a. Ripple Fast lon Losses \\
5.2. Collective Effects \\
5.3. DT and Alpha Particle Physics \\
\hline
\end{tabular}


TABLE 8-2

SYNOPSIS OF DIII-D CONTRIBUTIONS

TO ITER LONG-TERM PHYSICS R\&D NEEDS

\author{
January 1993 Report \\ (Institutions Collaborating with GA are Noted in Parentheses)
}

\title{
1. Power and Particle Exhaust Physics
}

PH 1.1a Divertor Power Load Profiles. A database of divertor plasma parameters (heat flux, plasma temperatures, and densities, etc.) has been constructed using about $200 \mathrm{ELM} \mathrm{H-mode} \mathrm{discharges.}$ Correlations with heating. power, plasma current, toroidal field, and magnetic geometry have been obtained. Modeling these data with the LEDGE scrape-off layer (SOL) simulation code shows that the heat profile widths are consistent with $\chi_{e}=0.2-0.5 \mathrm{~m}^{2} / \mathrm{sec}$, while the peak values suggest $\chi_{e}$ is about an order of magnitude larger (more like that used in the ITER CDA). Using standard ITER assumptions in the code yields a predicted heat flux scaling which is more pessimistic than that obtained from the database. (LLNL, ORNL, SNLL, SNLA, UCLA)

PH 1.1b Impact of Divertor Geometry Variation. A scan of the X-point height was carried out and the variation of peak divertor heat flux and electron temperature was measured. We observed that $T_{e, \text { div }}$ decreased as the $X$-point was raised, but the peak heat flux increased due to compression of the magnetic flux surfaces away from the field null. These results agreed with predictions from the LEDGE code using the standard ITER CDA assumptions. (LLNL, ORNL, SNLA)

PH 1.1c Hot Spots On Plasma Facing Components. A careful study of peak heat flux onto divertor tile edges was carried out, and our results were compared with those from Joint European Tokamak 
GA-A21186; FY92 DIII-D Research Operations Annual Report

(JET). It was found that carbon blooms should not be expected in DIII-D given the good alignment of the tile edges ( $\leq 1 \mathrm{~mm}$ step heights) and the relatively low fraction of heating power reaching the target plates ( $\leq 25 \%)$. A design model for optimal shaping of divertor tiles was obtained. (LLNL)

$\mathrm{PH}$ 1.1e Impact of Fueling. Experiments with the divertor baffle bias ring show that particle flows to the divertor and pumping plenum can be strongly affected by driving currents in the SOL plasma. Biasing the strike point can increase the pressure in the divertor pumping plenum by as much as an order of magnitude. Operating with the divertor strike point under the baffle can inhibit the core plasma fueling by recycling and lower the line-average density. (LLNL, ORNL, SNLA)

PH 1.1f ELMs and Other Edge Transients. The effect of ELMs on radiative divertor operation have been examined. In cases where the plasma is nearly detached from the divertor in the quiescent period between ELMs, the strong increase in parallel power flow is sufficient to reattach the plasma to the target plates. This lasts for only a few milliseconds, however. In general, the gas puff used to make the divertor highly radiative increases the ELM frequency and lowers the impurity content in the core plasma. (LLNL, ORNL, SNLA, UCLA)

PH 1.2 Impurity Radiation and Transport in the Bulk. SOL, and Divertor Plasmas. The question of whether it is the SOL or divertor which is the major source of core impurities has been partly answered by the fact that even in the VH-mode discharge, there remains a certain concentration of impurities in the plasma core which can be attributed to the cross-field transport of impurity ions from the divertor target plate. This fraction is relatively low, but without sufficient SOL screening, the wall source can surpass the divertor source and lead the plasma to a radiative collapse. (LLNL, ORNL, SNLL, SNLA, UCLA) 
PH 1.2a Powerfully Radiating Plasma Edge. More radiative divertor experiments have been carried out and analyzed. We find that divertor gas puffing can cool the plasma at the strike points to less than $10 \mathrm{eV}$ and reduce the peak heat flux by a factor of two or more. Analysis shows that this reduction is the result of increased radiative losses from the divertor region. Further cooling and heat flux reduction (up to factors of five or more) is associated with MARFE formation near the X-point. (LLNL, ORNL, SNLA)

$\mathrm{PH}$ 1.3 Exhaust of Helium and Hydrogen. Time-dependent $\mathrm{He}^{2+}$ density profiles have been measured in DIII-D plasma discharges during short He puffs into the plasma. Results from ELMing $H$-mode discharges show that giant ELMs purge helium from the plasma edge $(\rho>0.5)$, with the helium being then observed in the divertor region. (ORNL)

PH 1.4 Active Control and Optimization of Divertor. Divertor bias was used to increase the density of the SOL, which reduced the influx of impurity atoms across the separatrix. It was also found that the very high confinement $\mathrm{VH}$-mode has a natural dense $\mathrm{SOL}$, which was identified by modeling as the most likely explanation of the low impurity content of VH-mode plasmas. It appears that the divertor target peak heat flux can be reduced by local recycling at the gap between the plasma and the inner wall, which might offer a simple power management technique. An open radiative divertor was produced by deuterium puffing alone, and peak divertor power was reduced several fold. Radiation is from a MARFE near the X-point. The MARFE absorbs most of the energy burst of repetitive ELMs, thereby further protecting the divertor. The divertor electrode initiated and sustained a low-current tokamak plasma noninductively. (LLNL, ORNL, SNLA)

PH 1.5 Wall Conditioning Methods. Analysis using the REDEP code indicated that redeposition would reduce the peak erosion of the DIII-D divertor tiles by a factor of 6 at the outer strike point and by a factor of 9 at the inner strike point. Analysis of 12 DIII-D divertor tiles, exposed to the 
plasma for eight months, indicated that the magnitude of net erosion was comparable to the modeling predictions. (SNLL)

\section{Disruption Control and Operational Limits}

PH 2.1 Characterization of Disruptions. A disruption database has been created for DIII-D which presently contains 3400 shots. The database contains data on the current decay rates, density behavior, vessel motion, and loop voltage during disruptions as well as extensive information on plasma shape, safety factor, vertical stability parameters, and other equilibrium parameters immediately preceding the disruption and during the nondisruptive phase of the discharge.

PH 2.1a Disruption-Produced Runaways. A recent survey of hard $X$-ray production during DIII-D disruptions has failed to indicate the presence of any runaway electron production, in agreement with earlier statements conceming the lack of runaways in DIII-D disruptions. Previous simulations performed by Sandia National Laboratory, Albuquerque (SNLA) indicated that the high density in DIII-D disruptions was a significant factor in reducing the runaway production. Recent data indicates that the large density increase observed in DIII-D disruptions precedes the end of the thermal quench and should have the expected effect of inhibiting runaway production. (SNLA)

PH 2.2 Plasma Motions During Disruptions. Benchmarking of the Tokamak Simulation Code (TSC), including the plasma halo model, against a vertical displacement event in DIII-D has been successfully completed. DIII-D disruptions have also been simulated using the DINA 1-1/2 D resistive magnetohydrodynamic (MHD) simulation code in a collaborative effort with scientists from TRINITI laboratories in Russia. The code has been applied to the analysis of plasma motion during disruptions and has been instrumental in developing a predictive model for vertical motion of singlenull plasmas during disruptions. (ORNL, Kurchatov Inst.) 
PH 2.3 Disruption Avoidance and Control. Using both theory and results of error field/locked mode experiments on DIII-D, JET, and COMPASS, the critical $m=2, n=1$ applied error field for locked mode instability in ITER was calculated for ohmic, neutral beam, or if heated discharges. Ohmic discharges are predicted to be most sensitive because of their low rotation speed, but even co-injected neutral beam discharges (at normalized beta $=3$ ) will require keeping the relative error field well below $1 \times 10^{-4}$.

PH 2.4a Profile Effects on the Beta Limit. Highly peaked current density profiles with high internal inductance, $\ell_{j}$, have been produced by either a fast change in the plasma poloidal cross section or by a rapid change in the total plasma current. Both the confinement and the maximum achievable $B$ increase with $\ell_{i}$ and decrease as the current profile relaxes. Normalized toroidal beta, $B_{N}$. greater than $6 \%$ m-T/MA and values of the product $B_{N} \tau_{E} / \tau_{\text {ITER-89P }}$ greater than 15 have been obtained. (ORNL)

PH 2.4b Steady-State Pressure and Current in Inductive Operation Profile. DIII-D plasmas are more robust to external nonaxisymmetric error fields as the plasma fluid rotation is increased, but more sensitive as beta is increased. A larger critical error field is needed to nonlinearly induce instability in discharges rotating more rapidly. The beta limit in tokamaks can be reduced by locked modes nonlinearly induced by modest error fields. (ORNL)

PH 2.4c MHD Impact on High Beta Operations. Experimental studies of toroidal Alivén eigenmodes (TAE) modes indicate that the threshold value for fast ion beta for destabilization is an order of magnitude larger than initial estimates which considered only electron Landau damping. Theory and experimental results indicate that current profile control can help to stabilize the TAE modes through the influence of the magnetic shear on the continuum damping and electron kinetic damping. (UC Irvine) 
GA-A21186; FY92 DIII-D Research Operations Annual Report

PH 2.5 Density Limit. For a large range of DIII-D Ohmic, L-mode and ELMing H-mode discharges, the DIII-D density limit is well described by the Greenwald density scaling limit. Density limits in Ohmic and L-mode discharges are "hard" disruptions, which are typically triggered by the locking of a 2/1 MHD mode. In ELMing H-mode discharges, the density limit does not directly lead to a plasma termination, but instead is characterized by a return to $\mathrm{L}$-mode, with the $\mathrm{H}-$ to $\mathrm{L}$-mode transition occurring at densities at or slightly lower than the L-mode density limit. (LLNL, ORNL)

\section{Enhanced Confinement}

PH 3.1 Steady-State Operation with Enhanced Confinement. Further experiments and analysis of $\mathrm{VH}$-mode plasmas indicate that the improved confinement in $\mathrm{VH}$-mode is consistent with the increased penetration of the region of $E \times B$ velocity shear into the plasma from the plasma boundary, which then results in a larger region of turbulence suppression. (UCLA, Jülich GmBH)

PH 3.3 Transport Mechanisms. The presence of net inward electron energy transport during offaxis ECH was demonstrated under a variety of conditions. The error analysis in the best diagnosed shots gives an inward flux to an accuracy of three standard deviations. A working database has been compiled to search for dependence of the inward flux and to test theoretical models. (LLNL, ORNL)

\section{Optimization of Operation Scenarios and Long-Pulse Operation}

PH 4.1 Long-Pulse Operation. Current drive experiments were performed with fast wave power levels up to $1.2 \mathrm{MW}$ with co-current drive. Up to $40 \%$ of the $0.4 \mathrm{MA}$ plasma current is sustained noninductively. Counter-current drive produces highly peaked electron temperature profiles $\left[T_{e}(0) \leq 6 \mathrm{keV}\right]$ but much smaller noninductive currents. (ORNL) 


\section{Physics of a Burning Plasma}

PH 5.1 Fast lon Single-Particle Physics. The absolute magnitude of the fusion-product burnup is consistent with classical theory in high field $\left(B_{T} \gtrsim 1 \mathrm{~T}\right)$ discharges. However, large sawteeth can cause measurable reductions in burnup, although the net transport associated with the sawtooth is relatively modest $\left(\sim 0.1 \mathrm{~m}^{2} / \mathrm{sec}\right)$. Fishbones and TAE modes can effectively eliminate the confinement of $\mathrm{MeV}$ ions (equivalent diffusion coefficients $\$ 1.0 \mathrm{~m}^{2} / \mathrm{sec}$ ). (UC Irvine)

PH 5.2 Fast lon Collective Effects. In power scans at various values of toroidal field, the fast ion beta is found to saturate at $B_{f} \sim 1.2 \%$. This saturation is accompanied by a rapid rise in the observed TAE mode amplitude, suggesting that the instability has a threshold in $B_{f}$ and expels fast ions to maintain $B_{f}$ near the threshold value. (UC Irvine) 
SECTION 9

\section{TPX SUPPORT}





\section{TPX SUPPORT}

During 1992, General Atomics (GA) and the DIII-D staff provided support for the Tokamak Physics Experiment (TPX) preconceptual design effort on a wide variety of subjects. In addition, early in the year, staff members participated in the New Initiatives Task Force process which led to the definition and selection of the steady-state TPX concept. The technical support provided to TPX can be separated into the categories of physics and machine operations, divertor engineering and diagnostics, and component engineering (other than divertor). At the end of FY92, GA was awarded the contract for engineering of the divertor and plasma-facing components, and work in these areas has been transferred to that contract.

\subsection{PROGRAMMATIC ACTIVITIES}

Early in the year, J. Wesley served as an active member of the national New Initiative Task Force, assessing and evaluating proposed options for the New Initiative facility. After consideration of a range of options, including advanced tokamak physics and high performance options (as well as copper and superconducting coil variants), the task force recommended proceeding with the SSAT (now called TPX), a superconducting coil tokamak intended to address issues of steady-state, advanced tokamak operation. R. Callis also contributed to the review of the last surviving $5 \mathrm{~T}$ copper-coil proposal. P. Politzer participated as a GA representative in the SSAT definition and development activities and coordinated the GA contributions to these studies. GA hosted a number of meetings and workshops during the year.

\subsection{PHYSICS AND MACHINE OPERATIONS}

A. Turnbull carried out magnetohydrodynamic (MHD) stability calculations for nonsymmetric (single-null) TPX equilibria, taking advantage of the unique capability of the GA stability codes which do not assume up/down symmetry. An informal workshop on the 
problems of vertical stability was held at GA covering questions of approaches to plasma control, calculation techniques, and requirements for stability.

G. Jackson and W.P. West made recommendations on the wall conditioning techniques and scenarios for TPX. Besides $350^{\circ} \mathrm{C}$ bakeout, it was recommended that helium conditioning between discharges should be incorporated and that the tokamak should have the capability to be boronized. We also recommend that "real-time" impurity control be tried by injection of diborane during a plasma shot.

P. Politzer developed a four-year operating plan for TPX, incorporating the initial machine startup and the first advanced tokamak and divertor study campaigns. This was used to determine a $2.45 \mathrm{MeV}$ neutron production profile for input into the activation calculations.

\subsection{DIVERTOR STUDIES}

The principal contributions were definitions of the required divertor diagnostics and of diagnostic access requirements for the divertor. W.P. West developed a list of proposed divertor diagnostics needed to ensure safe operation of the TPX machine on the basis of GA's experience with the DIII-D systems. A cost estimate of these systems was also prepared. N. Brooks, W.P. West, and A. Colleraine made a preliminary estimate of the required space allocation for diagnostic devices within the TPX vacuum vessel.

In order to improve the stability of the TPX plasma, the in-vessel passive plate structures were modified, resulting in a major impact on the installation, removal and servicing of the divertor modules. P. Anderson, L. Sevier, and others initiated work on conceptual schemes to accomplish these tasks. Ways of breaking a divertor module into two pieces, or alternately "folding" it up for removal from the vessel were investigated. C. Baxi, R. Boonstra, and K. Redler did a hypervapatron analysis to determine the feasibility of using such devices in the high heat flux regions of the divertor. C. Baxi and R. Boonstra also examined various tile attachment schemes, for mounting the graphite tiles to the passive plate structures within the TPX vacuum vessel, showing that the Compact Ignition Tokamak (CIT)-type concept will not work unless the footprint of the tile in contact with the cooled backing plate is significantly enlarged in area. In the present configuration, tile surface temperatures would reach around $1500^{\circ} \mathrm{C}$ compared with the required design limit of $1000^{\circ} \mathrm{C}$. 


\subsection{COMPONENT ENGINEERING}

R. La Haye provided specifications for the field error tolerance and for the error correcting coils on the basis of DIII-D experience and independent calculations.

E. Reis and H. Shatoff analyzed three possible support structures for the TF coil system. These would have to provide all seismic and load supports and also serve as the thermal insulators between liquid helium and room temperatures. They concluded that the heat leaks, seismic and load support problems are very modest, and that the optimum choice of support structure will be determined by space allocation problems.

J. Leuer carried out a study of the use of ferromagnetic shims to reduce the TF ripple in the TPX $2.25 \mathrm{~m}$ design. Use of iron shims would allow a reduction from 16 to $12 \mathrm{TF}$ coils.

A. Colleraine, R. Gallix, and others made extensive contributions to the cost review for the TPX baseline configuration, particularly in areas where we have direct experience from the construction of Doublet III and DIII-D, such as coil systems, vacuum vessel construction, plasma-facing components, and neutral beam modifications. 




\section{COLLABORATIVE EFFORTS}

\subsection{DIII-D COLLABORATION PROGRAMS OVERVIEW}

Fusion collaboration programs at General Atomics (GA) encompass a broad spectrum of activities ranging from those which result directly in work on the DIII-D tokamak to those in which DIII-D staff members travel to other fusion facilities in order to acquire new technical information or carry out specialized experiments required for the development of advanced concepts. These collaboration activities are not only economically effective but provide a tremendous degree of technical support for development and execution of DIII-D programs while encouraging exchanges which are needed to stimulate new ideas necessary for solving difficult technical problems. It is clear from the new DIII-D results presented in preceding sections of this report that collaborations with other fusion research laboratories, both in the United States and in foreign countries, have played a key role in the internationally acknowledged success of the DIII-D program.

\subsubsection{JAPAN ATOMIC ENERGY RESEARCH INSTITUTE (JAERI)}

JAERI scientists continued to participate in the DIII-D program. H. Matsumoto worked on the perturbative particle transport using modified ONETWO code during giant edge localized modes (ELMs). The analysis indicated that inward pinching has been found to be insignificant in the edge region of the H-mode plasma. H. Kawashima improved the Russian X-ray diagnostics and found that high energy electrons extend above 50 to $100 \mathrm{keV}$ during electron cyclotron current drive (ECCD) and fast wave current drive (FWCD) experiments. S. Konoshima joined DIII-D near the end of May 1992 as a participant in the long-term assignment of JAERI personnel at DIII-D. He is analyzing the data of plasma shaping experiments on VH-mode and found the energy confinement more seriously affected by nickel contamination in lower triangularity case due to the interaction with outer walls. 


\subsubsection{NATIONAL LABORATORIES}

\subsubsection{LLNL/DIII-D Collaborations in FY92}

Lawrence Livermore National Laboratory (LLNL) personnel participated in both the edge physics and the advanced tokamak areas of research on DIII-D during FY92. Three LLNL scientists and two technicians resided in San Diego.

LLNL work in the edge physics and divertor area has included both experiments and modeling. In the area of basic divertor physics studies, scalings of scrape-off physics parameters have been developed and published. LLNL has continued to operate several divertor diagnostics, and we have started construction of a second infrared (IR) camera location to measure the toroidal symmetry of the divertor heat flux. LLNL participated in the design and installation of a new divertor bolometer system. The multicamera video digitizing system is operational and data analysis routines are being written. Work on the BNDY edge database has been progressing well, and over 350 time slices of DIII-D data are currently in the database. This has proved to be an important tool in developing the scaling studies discussed above.

In the area of modeling, substantial progress has been made in the development of the LLNL LEDGE code. The goal of this work is to develop a fully benchmarked edge code which can guide future experiments and the design of advanced divertors. A computational grid generator now exists called LGRID which can set up the mesh for LEDGE or DEGAS directly from the equilibrium codes EFIT or TEQ. Thus, LEDGE can easily be used for calculations on a particular DIII-D shot. The LEDGE code uses an implicit numerical approach which should be numerically stable; this enables the addition of cross field drifts and impurities to the model. During the last year, we have included drift terms such as the $\mathrm{E} \times \mathrm{B}$ drift in LEDGE; these seem to at least partly explain the in/out asymetries in the divertor heat flux measured by the IR camera. Edge transport coefficients have been derived from comparisons of the LEDGE model with experimental data; we find that $\chi_{\mathrm{e}}$ is in the range of 0.2 to 0.5 , and the perpendicular diffusion coefficient $D_{\perp}$ is 0.03 to 0.25 . We have also added improved models of the hydrogenic neutrals in LEDGE; two species are included: (1) a charge-exchange component (with energy comparable to the ion temperature), and (2) a Franck-Condon component (a few electron volts). In the future, we plan to link the DEGAS Monte-Carlo neutrals code with LEDGE for even more precise treatment of the neutral transport in a particular geometry. 
To more effectively model radiative divertor experiments, impurity radiation has been added to the LEDGE code. This was performed in two steps: first, a post-processor where the radiation was not coupled into the electron power balance; and second, a self-consistent treatment where the radiation is part of the power balance as the LEDGE code is being run. The impurity radiation is calculated with a version of MIST that accounts for chargeexchange recombination from neutrals and the impurity confinement time. The normal DIII-D divertor conditions (high $\mathrm{n}_{0}$, short impurity lifetime) cause substantial deviations from coronal equilibrium, resulting in enhanced radiation. An impurity density of a few percent can explain the observed 2 to $3 \mathrm{MW}$ reduction in divertor heat flux for radiative divertor experiments on DIII-D.

In the advanced tokamak area, LLNL has concentrated on the study of the plasma current density profile $j(r)$ with the motional Stark effect (MSE) diagnostic. This multichannel system is now operational and has obtained data during several DIII-D experimental runs. Calibrations to correct for the toroidal and vertical fields have been obtained. We observe that the on-axis safety factor $q_{0}$ is nearly unity, in contrast to results on other machines. MSE data from current-ramp and other confinement experiments is in progress. In the area of transport, LLNL has continued to develop tools to analyze heat propagation experiments with electron cyclotron heating $(\mathrm{ECH})$ and sawteeth.

\subsubsection{ORNLDIII-D Collaborations in FY92}

During FY92, Oak Ridge National Laboratory (ORNL) has made contributions to the DIII-D program in the following areas: high-beta tokamak optimization studies, experimental scaling studies of the divertor baffle pressure and preparation of the divertor cryopump, helium transport studies in various confinement regimes, disruption studies, fast wave heating and current drive, and preparation of the Joint European Tokamak (JET) pellet injector for operation on DIII-D. C. Klepper and D. Hillis completed one-year assignments in April and August 1992, respectively. R. Maingi began a one-year assignment in September 1992. Present onsite staff members are E. Lazarus, M. Menon, M. Wade, and R. Maingi. The radio frequency (rf) and pellet programs are covered by short-term visits.

\section{Advanced Divertor Program}

For more detailed pressure measurements, a set of ASDEX gauges and baratrons has been prepared for operation with the divertor cryopump. In 1993, there will be five ASDEX 
gauge heads mounted on the machine: three under the baftle (two at separated toroidal locations), one in the private flux region, and one on top of the baffle via a chimney. There are also two baratrons on the machine: one in the private flux region and one under baffle as part of the Penning gauge helium detection system. The ASDEX gauges will provide fast time response (several milliseconds).

Also in preparation for the pumped divertor experiments, the divertor spectrometer was upgraded to a multichord system. With the newly calibrated photodiode array, simultaneous measurements of hydrogen and helium recycling in the divertor have been obtained. Absolute photon fluxes have been obtained which will be used to improve the results of divertor modeling.

The neutrals model in the standard version of the edge plasma transport code B2 has been improved with the addition of molecular desorption at the neutralizer plates. The model now includes both the back-scattered atomic component and the desorbed molecular component with the ratio of the two computed from the Eckstein-Verbeeck reflection coefficients. The molecular physics in the model includes the Franck-Condon processes as well as the particle and energy sources and sinks for the electron and ion fluids. The new model includes very good approximations for the electron power loss based on data of Janev. The ion power source associated with charge exchange and the contribution of the Franck-Condons is also included. Comparison of the results of the new B2 neutrals model with coupled B2/DEGAS calculations show very good agreement in simulations that use the flux surface geometry from DIII-D shot 70619. The new model is presently being compared in detail with B2/DEGAS in various parameter regimes. In particular, the analysis of DIII-D baffle pressure data will require the improved neutrals model and the speed of the B2 stand alone code.

A track length estimation technique has been developed and implemented in the DEGAS code. It significantly reduces the time required to track neutral particles (particularly wall temperature molecules) in vacuum regions having low conductance to the plasma or to pumps. The new technique eliminates short track lengths when the particle is in vacuum. Typically, a factor of 5 to 6 improvement in running time has been observed in baffle neutral pressure simulations. This improvement will permit a much more rapid analysis of pressure and pumping experiments with the Advanced Divertor Program (ADP) cryopump. 
The collaborative program on He transport and exhaust provides critical data on the removal of He ash for the International Thermonuclear Experimental Reactor (ITER). Experimental studies on DIII-D in the past year have focused on determining the transport properties of helium during a wide variety of diverted discharges with special emphasis placed on ELMing $\mathrm{H}$-mode discharges and the scaling of these properties with energy confinement. Transport coefficients are determined by measuring the He profiles and modeling the transport with the MIST code (developed by R. Hulse, Princeton Plasma Physics Laboratory). The transport coefficients that reproduce the data taken during L-mode discharges agree reasonably well with those found in low confinement regimes in TEXTOR, Tokamak Fusion Test Reactor (TFTR), and JT-60. Since it has been observed that ELMs purge a significant amount of helium from the plasma edge, experiments have been conducted over a wide range of ELM frequencies ( 0 to $120 \mathrm{~Hz}$ ) at constant plasma current to determine the effect of ELM frequency on helium transport. The MIST modeling results indicate that the quantity $1 /\left(D_{\alpha} \tau_{\mathrm{E}}\right)$ (which is an approximate measure of $\tau_{\mathrm{He}} / \tau_{\mathrm{E}}$ ) decreased by a factor of two as the ELM frequency increased from 0 to $120 \mathrm{~Hz}$. This result indicates that increasing the ELM frequency is certainly beneficial in increasing helium transport relative to energy transport though conclusions on the level of ELM frequency that is sufficient to avoid quenching of ignition in ITER cannot be made at this time.

An example of a radial He profile just before and during a giant ELM event for a typical DIII-D plasma with giant ELMs is shown in Fig. 10.1-1. For the period just before the ELM, the He and electron density profiles are that near the plasma center and steep near the separatrix. During the giant ELM, the He density profile indicates that $\mathrm{He}$ is lost from the edge plasma for radii of $p>0.5$, while the profile is not affected for $p<0.5$. The He photomultiplier signals in the divertor show that when the He is purged from the plasma edge, the $\mathrm{He}^{+}$line emission from the divertor increases abruptly, suggesting a sharp increase in the divertor He content. This is a clear demonstration that ELMs purge He from the edge of the core plasma. If pumping were provided in the baffle region of DIII-D, this He would most likely be removed. As part of the ADP on DIII-D, a cryopump will be installed at the end of 1992 which can be conditioned with an argon frost layer to provide He pumping.

ORNL participated in the divertor cryopump optimization which is discussed in Section 6.2.2. 


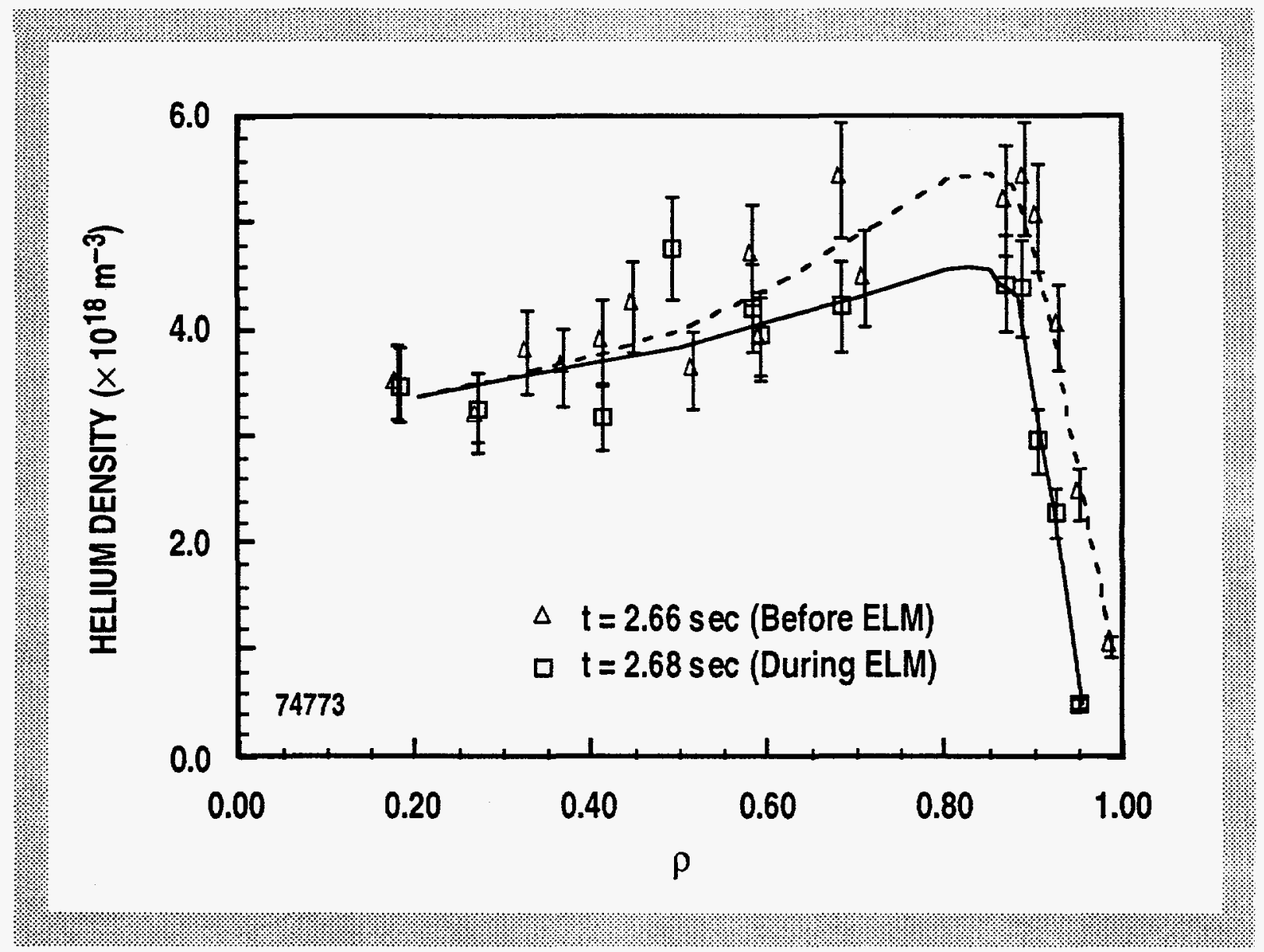

Fig. 10.1-1. Helium density profile as measured by CER just before and during a giant ELM.

\section{Plasma Disruption Studies}

Two different issues were investigated: (1) distribution of poloidal current flow in tokamak vessel walls during plasma disruptions, and (2) voltage transients induced on the torus as well as internal components of the tokamak during plasma disruptions.

Large currents, estimated to be up to $20 \%$ of the plasma current, flow through the tokamak vessel walls during vertical displacement episodes (VDEs) and during major disruptions. In order to predict with more confidence the resulting maximum local forces on the vessel which are likely to occur, more detailed information on the spatial distribution of the currents is necessary. Direct measurements of current flow through the walls were carried out at different poloidal and toroidal locations on the tile current arrays that were 
installed in DIII-D. A study of the signals suggests that, on a submillisecond time scale, the currents are not toroidally symmetric. The asymmetry is, in general, greater for major disruptions than for VDEs.

Based on measurements using flux loops and magnetic probes, the voltage transients at different locations of the tokamak resulting from VDEs and major disruptions were estimated. The voltages produced under a wide range of plasma conditions, comprising 216 major disruptions and 34 VDEs, were examined. The loop voltages induced at the top and bottom of the vacuum vessel and on a toroidal cryopump loop, planned to be located adjacent to the lower outer divertor strike region, were estimated. The induced voltage was a function of: (1) predisruption plasma current, (2) the toroidal field, (3) the maximum rate of change of plasma current, and (4) the proximity to the region where plasma contacts the wall. A scaling law that predicts an upper limit for the disruption-induced voltage in DIII-D was obtained. Since the induced voltage is affected by the proximity to the plasma wall contact region, in general, there is large asymmetry between the voltages induced at the top and bottom regions of the tokamak. This asymmetry is largest in the case of VDEs (up to about $400 \%$ ) while radial disruptions, where the movement of the centroid of the plasma is radial, exhibit good up/down symmetry.

\section{RF Heating and Current Drive}

There were three main activities in the FWCD collaboration during 1992: (1) operation of the existing four-strap antenna in Faraday shieldless configuration, (2) fabrication of a new single-tier $\mathrm{B}_{4} \mathrm{C}$ coated Faraday shield, and (3) design of two new long-pulse FWCD antennas. The DIII-D FWCD program is described in Section 3.3, and the operation in shieldless configuration is described in Section 3.3.1.

The new Faraday shield for the existing four-strap antenna should improve its performance substantially. The new shields have only a single tier of rods, tilted at $12^{\circ}$ to match the pitch of the magnetic field lines at the antenna, for increased plasma loading and, hence, higher power handling capability. The new shield has a plasma-sprayed coating of boron carbide to reduce impurities and incorporates a microwave reflectometer in collaboration with the University of California at Los Angeles (UCLA) to provide edge density profiles in front of the antenna. The shield was sent to Tore Supra (Cadarache, France) for plasma spraying with $\mathrm{B}_{4} \mathrm{C}$. 
Conceptual design of two new long-pulse $120 \mathrm{MHz}$ four-element antenna arrays for installation in the $0^{\circ}$ and $180^{\circ}$ ports began in March. Each antenna will consist of four independent modules, each of which is designed to fit through a standard DIII-D midplane port. A conceptual design review was held on April 29, and an intermediate design review was held on September 17 at GA. The final design review is scheduled for January 1993 at ORNL.

\section{Pellet Injector}

A project to install a pellet injector on DIII-D was initiated this year. The primary goals of the experiments using the injector are continuous fueling inside the separatrix with the pumped divertor, increased plasma performance (PEP mode) as found on JET, and repetitive perturbations to the density profile to investigate plasma transport. Installation on DIII-D will occur in FY94.

The injector will be the three-barrel, repeating pneumatic injector installed on the JET tokamak in 1987. The injector is capable of injecting more than $852.7 \mathrm{~mm}$ pellets at up to $1.3 \mathrm{~km} / \mathrm{sec}$. Deuterium will be used for the pellets and the propellant gas. A conceptual design review of the DIII-D pellet injector was successfully completed at GA in September 1992.

\section{High Beta Tokamak Optimization Studies}

Over the past year, there has been a substantial attempt to characterize the role of plasma shape in determining the quality of confinement. In part, this work was motivated by our inability to obtain $\mathrm{VH}$-mode levels of confinement time in the usual single-null plasma shape. This difference correlates well with (up/down) averaged triangularity, rather than whether the plasma is single or double null. This difference will remain a topic of research on DIII-D.

A more general measure of tokamak performance is the product $B \cdot \tau$. An examination of the DIII-D database indicates that the highest $B \cdot \tau$ values occur in double-null plasmas where $B \cdot \tau$ reaches $1.4 \% \cdot \mathrm{sec}$, about twice those values occurring in single-null plasmas. In double-null diverted ELM-free plasmas, we find $\tau_{\mathrm{E}}$ values of approximately three times ITER-89P scaling are maintained by $B_{N}$ values greater than 3 . However, for single-null diverted ELM-free plasmas, we find that confinement degradation beginning at $B_{N} \approx 2$ limits the achievable $B \cdot \tau$. For both shapes, we find that $B \cdot \tau$ values decrease for $q_{95}>3.3$. 
GA-A21186; FY92 DII-D Research Operations Annual Report

\subsubsection{UNIVERSITY PROGRAMS}

University collaborations continue to play an important role in the DIII-D research program. A number of the important diagnostic systems on DIII-D were designed and installed as a result of these collaborations. In addition, university collaboration coordinators, along with their post doctorates and graduate students, support DIII-D's research goals by operating their diagnostics and analyzing the data. In addition, university collaborations continue to provide strong numerical and theoretical support for the DIII-D experimental program.

During FY92, the UCLA maintained the largest university collaboration program on DIII-D. Other significant university collaborations included: the University of California at Berkeley (UCB), the University of California at San Diego (UCSD), the University of California at Irvine (UCI), the University of Maryland (UM), and Massachusetts Institute of Technology (MIT).

UCLA collaborations with the Institute for Fusion Plasma Research (IFPR) are organized into two research areas. Professor R. Conn's group works with the fast stroke Langmuir probe, in conjunction with Sandia National Laboratory, Albuquerque (SNLA), making measurements of the boundary and scrape-off layer (SOL) turbulence. These measurements are done in collaboration with DIII-D's divertor physics and L-H transport groups. A second UCLA group, coordinated by T. Peebles and supported under DOE-APP and a DIII-D subcontract, works with an far-infrared (FIR) laser scattering diagnostic and an rf reflectometer studying turbulent transport in DIII-D. This group is responsible for the reflectometer and the FIR scattering system.

Professor T.K. Fowler's UCB group worked on L-H transport modeling, a drift orbit code, LEDGE code support for boundary and SOL modeling, and helium transport studies. Their staff supported DIII-D Boundary Layer Division experiments with their work on L-H transport modeling and worked on a drift orbit code and the implementation of new effects in the LEDGE code. D. Finkenthal worked on helium transport.

UCSD collaborations involving Profs. M. Rosenbluth and P. Diamond continued to support the DIII-D program with numerical and theoretical studies of Toroidal Alfvén Eigenmodes (TAE) and trapped ion turbulence modeling. Graduate students worked on a fluid-like moment representation of turbulence and on TAE theory with M. Rosenbluth.

UCI collaborations have continued with Prof. W. Heidbrink and his graduate student on TAE experiments and triton burnup. From MIT, Prof. M. Porkolab participated in the DIII-D Electron 
Cyclotron Heating (ECH) and Fast Wave Current Drive (FWCD) programs with a graduate student working on the Phase Contrast Imaging System. A University of Maryland (UM) collaboration, under the direction of Prof. R. Ellis, continues to be responsible for the DIII-D vertical electron cyclotron emission (ECE) diagnostic. A UM post-doctorate has operated the ECE system since May of 1992. Prof. A. Hassam, of the University of Maryland, arrived at GA late in FY92 for a one-year assignment involving work on an L-H transition theory.

\subsection{INTERNATIONAL COOPERATION}

GA's International Collaboration Program continues to provide a broad source of innovative new ideas and opportunities which support the DIII-D research program. Major collaborations are underway with JET in England, Tore Supra in France, ASDEX-U and TEXTOR in Germany, and JT-60U in Japan. In addition to the benefits gained from DIII-D staff assignments in these laboratories, foreign scientists visiting GA have made significant contributions to the DIII-D program goals.

There were a total 35.5 man months of onsite foreign collaborator months at DIII-D in FY92. Contributions to the DIII-D program goals were made by; H. Zohm (3-month assignment) from ASDEX-U working on ELM studies, C. Lowery (5-month assignment) from JET working on an analysis of carbon bloom thresholds, F. Allais (11-month assignment) from the University of Paris working on magnetic island effects, L. Langyel (1-month assignment) from ASDEX-U working on pellet ablation modeling, S. Konoshima (4.5-month assignment) from JT-60U working on DIII-D confinement studies, A. Astapkovich (3-month assignment) from the Efremov Institute in Russia working on numerical simulations of disruptions, V. Lukash (3-month assignment) from the Troitsk Institute working on disruption studies, O. Sauter (1-year assignment) from Switzerland developing a Fokker-Planck code, and Y. Martin (1-year assignment) from Switzerland working on charge exchange recombination (CER). In FY93, foreign collaborators are scheduled for more than 78 man months of onsite time.

A summary of the progress made by DIII-D staff members in support of the International Collaboration Program is given below. 


\subsubsection{JET}

JET collaborations continue to be the largest part of GA's international fusion research activities making up 36\% (20.4 JET assignment months) of the total International Collaboration Program off-site work. Tasks within the JET Collaboration centered primarily around advanced divertor design issues. C. Baxi and T. Hodapp supported this work by sharing a year-long assignment at JET. Hodapp worked on an advanced divertor water cooling system which is relevant for future divertor designs. C. Baxi carried out a detailed analysis of the water-cooled hypervaportron design used as a neutral beam dump in JET and developed a method of predicting its thermal performance, which was in good agreement with the experimental results.

Physics collaborations connected with the assignments of T. Carlstrom, R. La Haye, and C. Greenfield were primarily oriented around improved confinement issues. T. Carlstrom, who worked principally on $\mathrm{H}$-mode issues, was present for the initial D-T operations in JET. R. La Haye worked on error field and locked mode characteristics in JET and compared them with the known DIII-D error fields. C. Greenfield arrived in August to study VH-mode discharges in JET. Fifty-nine interesting discharges were identified. These discharges, which range in confinement from 1 to above 2 times JET/DIII-D H-mode confinement scaling, occur over a wide range of parameters and in configurations where VH-mode had not previously been observed on DIII-D. These studies have proven helpful in elucidating our understanding of $\mathrm{VH}$-mode physics.

\subsubsection{ASDEX-U}

ASDEX-U was just starting initial operations in FY92. Consequently, there were no significant long-term DIII-D staff member assignments on ASDEX-U. T. Luce and S-C. Chiu spent several months at the Max Planck Institute working on rf heating and current drive issues. P. Gohil spent the month of September in Garching working on boundary physics comparisons, and R. Waltz spent several months making theoretical comparisons between tokamaks and stellerators.

\subsubsection{TORE SUPRA}

The Tore Supra collaboration represents an opportunity for DIII-D personnel to study long-pulse, high-power if current drive and boundary control issues. In the area of if physics, 
R. Harvey spent several months at Tore Supra developing a Fokker-Planck code to model lower hybrid current drive (LHCD). An ECH transmission workshop was also held in Cadarache and progress on rf transmission and power-generating components continues.

In the area of boundary physics and control, G. Stabler made a brief visit to Tore Supra to discuss SOL theory. T. Evans spent one month at Cadarache continuing his work on ergodic boundary layer control during high-power LHCD discharges. A paper entitled "Magnetic Perturbation Effects on Boundary Plasmas During High Power Lower Hybrid Current Drive in Tore Supra" describing the results of this work was written for the Journal of Nuclear Materials.

S. Lippmann spent four months in Cadarache making spectroscopic measurements of poloidal asymmetries in ergodically diverted plasmas. The object of these experiments is to understand the structure of poloidal asymmetries in different operating modes and how they are influenced by intrinsic magnetic perturbations due to small random deviations in toroidal and poloidal field coil positions.

A scanning spectrometer was used to obtain line-integrated emissions. The data is compared to a model developed by Drs. Lippmann and Evans. Figure 10.2-1 shows the structure of the magnetic field in Tore Supra with the ergodic divertor coil energized. The outer midplane is located at poloidal angle $\mathrm{q}=0^{\circ}$ in this figure.

This figure was produced by integrating magnetic field line trajectories at 120 starting points 100 times around the tokamak using the TRIPND code. Each point in the plot represents the position of the field line as it passes through a poloidal cross section located at the toroidal position of the scanning spectrometer. The island structures seen in the TRIPND data are used as a source of poloidal modulations for the plasma temperature and density. These modulations are then included in a modeling code to predict trace-injected impurity concentrations and their poloidal emission distributions. Figure 10.2-2 shows the resulting Neon VII density and emission distribution with background plasma densities and temperatures modulated by the islands shown in Fig. 10.2-1. The modeling code is used to numerically integrate emission distributions such as that shown in the lower right hand corner of Fig. 10.2-2 and the output of the code is compared with experimental data from the scanning spectrometer. 


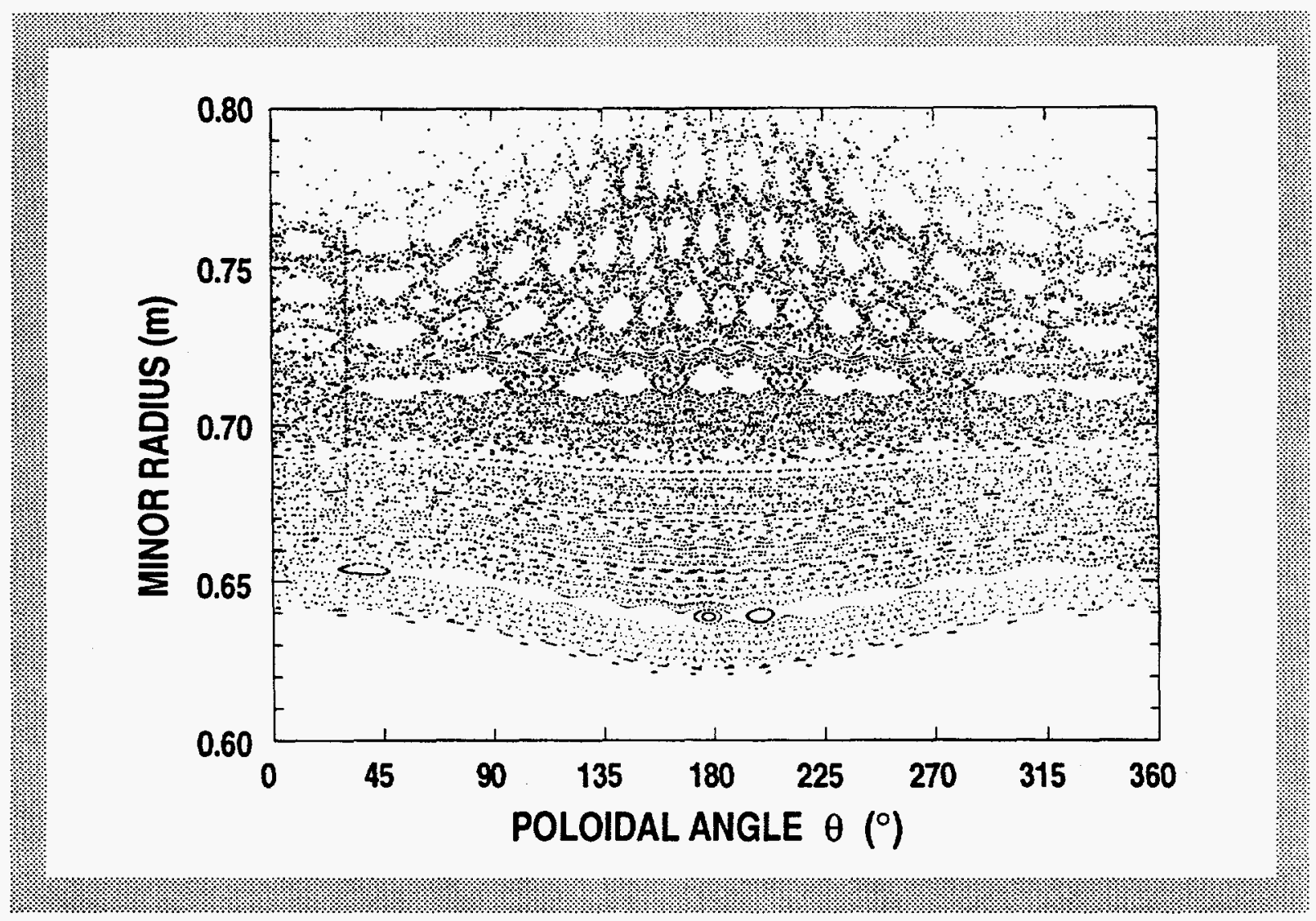

Fig. 10.2-1. Field line puncture plot showing the poloidal structure of the ergodic boundary layer in Tore Supra.

\subsubsection{JT-60U}

JT-60U was designed with the idea of achieving higher plasma performance levels than JET, TFTR, and DIII-D. Initial H-mode results have not been up to expectations. A number of new ideas are being tested in order to achieve this goal and foreign collaborations are considered an important source of innovative ideas to help sort out an explanation for their relatively short $\mathrm{H}$-modes with low enhancement factors.

There were two DIII-D staff assignments at JT-60U during FY92. T. Taylor spent four months in Japan working on techniques for improving JT-60U H-mode performance. During his assignment, $\mathrm{H}$-modes with enhancement factors of 1.44 and $0.5 \mathrm{sec}$ duration were obtained. Two factors which appear to be important for these higher performance $\mathrm{H}$-modes were lower wall temperatures and helium Taylor discharge cleaning between shots. The time evolution of these H-modes is somewhat different than those on DIII-D. Two distinct phases were identified after the first L-to-H transition signature. Some candidates for 
Fig. 10.2-2. Left half poloidal modulations in the background plasma parameters of Tore Supra due to the islands shown in Fig. 10.2-1. Pight half - resulting Neon VII density distribution and emission profiles.

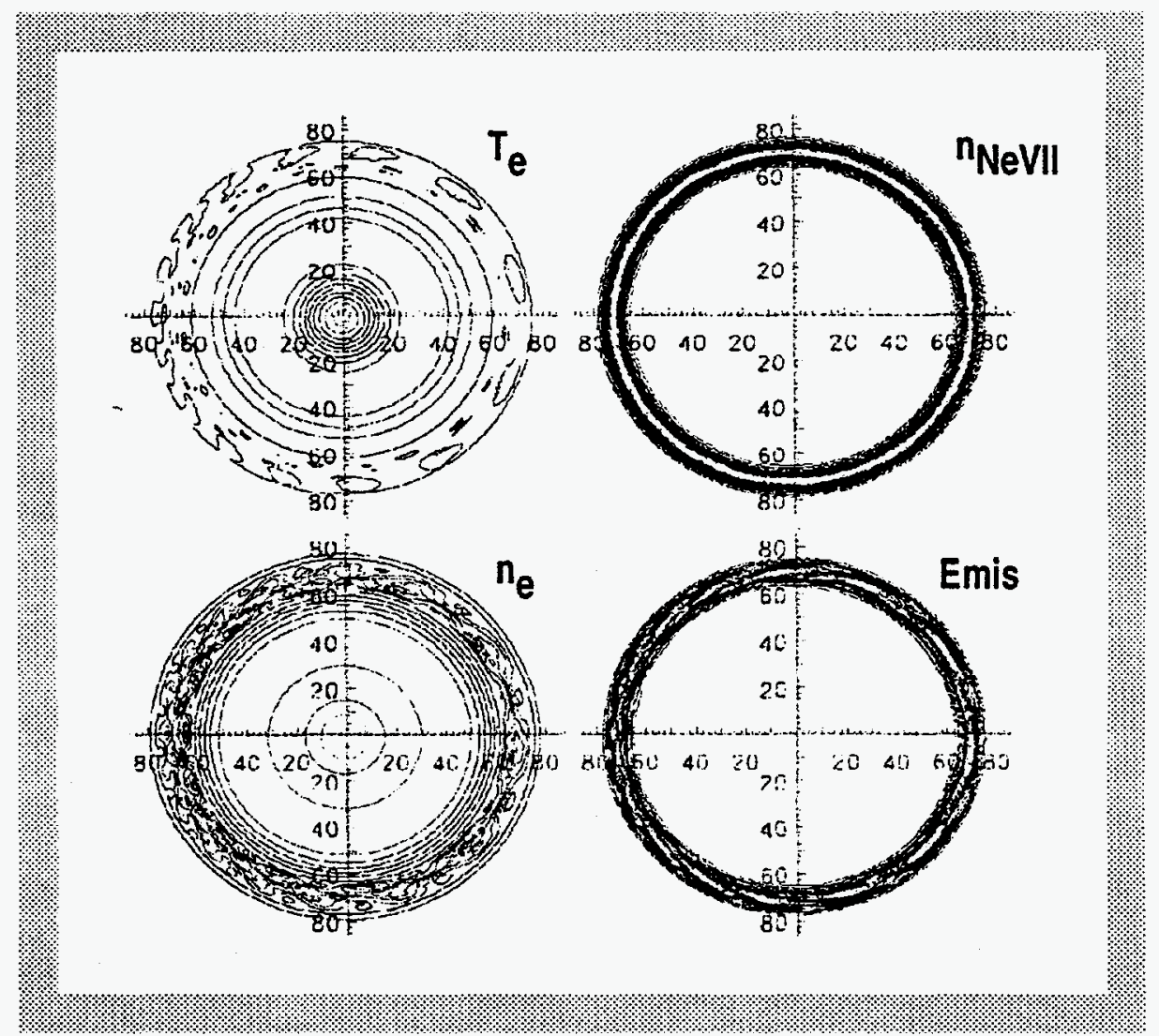

explaining JT-60U $\mathrm{H}$-mode results are: strong impurity effects (particularly due to oxygen), recycling effects, or the effects of toroidal ripple.

G. Jackson spent one month at JT-60U. He participated in the first boronization of the vessel and in plasma characterizations after the boronization. Immediately after boronization, JT-60U achieved a stored energy of $5.3 \mathrm{MJ}$ - the highest value ever obtained in the machine.

\subsubsection{TEXTOR}

Collaborations at TEXTOR were initiated for the first time in FY92. The primary activity within this collaboration was in the area of advanced wall coating materials and procedures. G. Jackson spent two months at TEXTOR participating in the first siliconization of the machine. This technique proved to be effective in reducing oxygen levels by a factor of 10 . It was also noted that helium pumping during increased due to the silicon film. 


\subsubsection{COMPASS-D}

The COMPASS-D tokamak has a unique set of resonant magnetic perturbation coils which can be used to control boundary instabilities and improve divertor operations. T. Evans made a short visit to COMPASS-D, JET, and START to propose experiments in this area. Dr. Evans had detailed discussions with many of the COMPASS staff members about machine capabilities and possible collaboration topics in the area of resonant magnetic perturbation research. Dr. Evans obtained engineering drawings of the COMPASS-D divertor and RMP coils for preliminary modeling studies. The COMPASS-D saddle coils will be added to the TRIPND field line tracing code in order to begin preliminary modeling work.

\subsubsection{TSP}

Two DIII-D staff members made short visits to the Troitsk TSP facility. W. West and $\mathrm{N}$. Brooks discussed materials issues and made an evaluation of a high-throughput, high-resolution visible spectrometer. D. Humphrys traveled to Russia for disruption work while J. Lohr, C. Moeller, and D. Remsen went for discussions on radio frequency hardware and related physics issues.

\subsubsection{T-10 ECH COLLABORATION}

The very successful collaboration between DIII-D and the T-10 group at the Kurchatov Institute in Moscow continued, and during 1992, the scope was expanded to include contract support for the ECH and ECCD programs on T-10. GA scientists were in Moscow to participate in the fall T-10 campaign and contributed to the planning, execution, and analysis of the experiments. The cooperation work has resulted in several jointly authored papers and has provided valuable data at high power densities and in parameter regimes which will be explored in the next series of ECCD and ECH experiments on DIII-D.

The T-10 campaign during the last quarter of 1992 demonstrated ECCD at the second harmonic resonance, good absorption and heating, discharges with a high fraction of bootstrap current, and an operating regime with the characteristics of the $\mathrm{H}$-mode. Boronization, using nontoxic carborane was successful at reduing $\mathrm{Z}_{\mathrm{eff}}$ to around 1.2 and a 
new X-ray diagnostic was successfully tested. Four new gyrotrons capable of producing greater than $500 \mathrm{~kW}$ at $140 \mathrm{GHz}$ were tested and used in the experimental program. Plans are currently underway for a continuation of the cooperation, with exchanges of personnel in both directions, during CY93. The joint work agreements for the $\mathrm{T}-10$ research have become a model for greatly expanding contacts beween the U.S. and the Russian fusion programs and for increased cross fertilization in other areas of high technoloyg as well. 
SECTION 11

\section{FY92 PUBLICATIONS}





\section{FY92 PUBLICATIONS}

Allais, F., and T.E. Evans, "Magnetic Island Dynamics Including Self-Consistent Plasma Responses and Small Resonant External Perturbations," in Bull. 34th Annual American Physical Society Meeting, November 16-20, 1992, Seattle, Washington, (Abstract) 37 (1992) 1569.

Allen, J.C., R.W. Callis, W.P. Cary, T.E. Harris, and A. Nerem, "Eight Channel-16 Bit, Bidirectional Analog to Digital Monitoring and Control System," in Proc. 14th Symp. on Fusion Engineering, IEEE (San Diego, California, September 30-October 3, 1991) Vol. II (1992) 844; General Atomics Report GA-A20604 (1991).

Allen, S.L., R. Cohen, D.N. Hill, W. Nevins, et al., "Concept Development of a Radiative Divertor for DIII-D," in Bull. 34th Annual American Physical Society Meeting, November 16-20, 1992, Seattle, Washington, (Abstract) 37 (1992) 1566.

Austin, M.E., R.F. Ellis, R.W. Harvey, and R.A. James, "Analysis of Nonthermal ECE Spectra from DIII-D Plasmas Using a Michelson Interferometer," in Bull. 34th Annual American Physical Society Meeting, November 16-20, 1992, Seattle, Washington, (Abstract) 37 (1992) 1567.

Baker, D.R., and R.J. Groebner, "Analysis of DIII-D CER Data Using a Neural Network," in Bull. 34th Annual American Physical Society Meeting, November 16-20, 1992, Seattle, Washington, (Abstract) 37 (1992) 1568.

Baxi, C.B., J.L. Doane, and D.L. Sevier, "Thermal Analysis and Design of Polarizer Mirrors for Waveguides in the DIII-D Tokamak," to be published in Proc. International Society for Optical Engineering; July 19-24, 1992, San Diego, California; General Atomics Report GA-A20972 (1992).

Baxi, C.B., and H. Falter, "Analytical Prediction of Thermal Performance of Hypervapotron and Its Application to ITER," to be published in Proc. 17th Symp. on Fusion Technology, September 14-18, 1992, Rome, Italy; General Atomics Report GA-A21035 (1992). 
Baxi, C.B., and H. Falter, "A Model for Analytical Performance Prediction of Hypervapotron," to be published in Proc. 5th International Topical Meeting on Nuclear Reactor Thermal Hydraulics, September 21-24, 1992, Salt Lake City, Utah; General Atomics Report GA-A20959 (1992) and JET-P(92)56.

Baxi, C.B., G.J. Laughon, A.R. Langhorn, K.M. Schaubel, et al., "Verification Test for Helium Panel of Cryopump for DIII-D Advanced Divertor," in Proc. 14th Symp. on Fusion Engineering, IEEE (San Diego, California, September 30-October 3, 1991) Vol. II (1992) 1226; General Atomics Report GA-A20589 (1991).

Baxi, C.B., W. Obert, "Optimization of Thermal Design for Nitrogen Shield of JET Cryopump," in Proc. 14th Symp. on Fusion Engineering, IEEE (San Diego, California, September 30-October 3, 1991) Vol. II (1992) 1218; General Atomics Report GA-A20587 (1991).

Bramson, G., "Timing System for Neutral Beam Injection on the DIII-D Tokamak," in Proc. 14th Symp. on Fusion Engineering, IEEE (San Diego, California, September 30-October 3, 1991) Vol. II (1992) 814; General Atomics Report GA-A20640 (1991).

Becoulet, A., and D. Moreau, "Variational Full Wave Calculation of Fast Wave Current Drive in DIII-D Using the ALCYON Code," General Atomics Report GA-A20846 (1992).

Brooks, N.H., A.M. Howald, C.C. Klepper, and W.P. West, "Multichord Spectroscopy of the DIII-D Divertor Region," Rev. Sci. Instrum. 63 (1992) 4941; General Atomics Report GA-A20872 (1992).

Brooks, N.H., A.M. Howald, W.P. West, and C.C. Klepper, "Multichord Visible Spectroscopy of the DIII-D Divertor," in Bull. 34th Annual American Physical Society Meeting, November 16-20, 1992, Seattle, Washington, (Abstract) 37 (1992) 1567.

Burrell, K.H., T.N. Carlstrom, E.J. Doyle, D.F. Finkenthal, et al., "Physics of the L-Mode to H-Mode Transition in Tokamaks," Plasma Physics and Controlled Fusion 34 (1992) 1859; General Atomics Report GA-A20993 (1992).

Burrell, K.H., P. Gohil, R.J. Groebner, and R.P. Seraydarian, "Radial Electric Field Structure in VH-Mode Plasmas in DIII-D," in Bull. 34th Annual American Physical Society Meeting, November 16-20, 1992, Seattle, Washington, (Abstract) 37 (1992) 1410.

Callis, R., W. Cary, C. Moeller, R. Freeman, et al., " $110 \mathrm{GHz}$ ECH Heating System for DIII-D," in Proc. 9th Topical Conf. on Radio Frequency, AIP (August 19-21, 1991, Charleston, South Carolina) Vol. 244 (1992) 24; General Atomics Report GA-A20627 (1991). 
Campbell, G.L., J.R. Ferron, E. McKee, A. Nerem, et al., "New DIII-D Tokamak Plasma Control System," to be published in Proc. 17th Symp. on Fusion Technology, September 14-18, 1992, Rome, Italy; General Atomics Report GA-A21036 (1992).

Carlstrom, T.N., G.L. Campbell, J.C. DeBoo, R. Evanko, et al., "Design and Operation of the Multipulse Thomson Scattering Diagnostic on DIII-D," Rev. Sci. Instrum. 63 (1992) 4901; General Atomics Report GA-A20849 (1992).

Carlstrom, T.N., J.C. DeBoo, R.E. Waltz, and J.P. Christiansen, " $\mathrm{r}$ * Scaling of Confinement in Dimensionally Similar Discharges in JET and DIII-D," in Bull. 34th Annual American Physical Society Meeting, November 16-20, 1992, Seattle, Washington, (Abstract) 37 (1992) 1410.

Carlstrom, T.N., G.L. Campbell, J.C. DeBoo, R.G. Evanko, et al., "The Multipulse Thomson Scattering Diagnostic on the DIII-D Tokamak," in Proc. 14th Symp. on Fusion Engineering, IEEE (San Diego, California, September 30-October 3, 1991) Vol. II (1992) 1195; General Atomics Report GA-A20614 (1991).

Cary, W.P., J.C. Allen, R.W. Callis, J.L. Doane, et al., "110 GHz ECH on DIII-D: System Overview and Initial Operation," in Proc. 14th Symp. on Fusion Engineering, IEEE (San Diego, California, September 30-October 3, 1991) Vol. II (1992) 912; General Atomics Report GA-A20632 (1991).

Chan, V.S., L.L. Lao, and T.S. Taylor, "Confinement and Performance Limits for Advanced Tokamaks," in Bull. 34th Annual American Physical Society Meeting, November 16-20, 1992, Seattle, Washington, (Abstract) 37 (1992) 1508.

Chiu, S.C., R.W. Harvey, Y. R. Lin-Liu, C.C. Petty, et al., "Simulation of FWCD with RF Codes," in Bull. 34th Annual American Physical Society Meeting, November 16-20, 1992, Seattle, Washington, (Abstract) 37 (1992) 1512.

Chiu, S.C., M.J. Mayberry, R. Pinsker, C.C. Petty, M. Porkolab, "Theory of Ion Bernstein Wave Coupling at Low Edge Densities," in Proc. 9th Topical Conf. on Radio Frequency (August 19-21, 1991, Charleston, South Carolina) p. 169; General Atomics Report GA-A20608 (1991).

Clow, D.D., D.H. Kellman, "Computer Control of the High Voltage Power Supply for the DIII-D Electron Cyclotron Heating System," in Proc. 14th Symp. on Fusion Engineering, IEEE (San Diego, California, September 30-October 3, 1991) Vol. II (1992) 831; General Atomics Report GA-A20606 (1991). 
Coda, S., M. Porkolab, K.H. Burrell, and T.N. Carlstrom, "Edge Fluctuation Measurements by Phase Contrast Imaging on DIII-D," in Bull. 34th Annual American Physical Society Meeting, November 16-20, 1992, Seattle, Washington (Abstract) 37 (1992) 1512.

Coda, S., M. Porkolab, and T.N. Carlstrom, "A Phase Contrast Interferometer on DIII-D," Rev. Sci. Instrum. 63 (1992) 4974; General Atomics Report GA-A20876 (1992).

Cuthbertson, J.W., D. Buchenauer, D.N. Hill, and A.H. Futch, "Measurement of Sheath Power Transmission to Divertor Surfaces and Electrically-Biased Probes," in Bull. 34th Annual American Physical Society Meeting, November 16-20, 1992, Seattle, Washington, (Abstract) 37 (1992) 1567.

DeBoo, J.C., C.M. Greenfield, K.H. Burrell, J.R. Ferron, et al., "Performance and Transport in VH-Mode," in Bull. 34th Annual American Physical Society Meeting, November 16-20, 1992, Seattle, Washington, (Abstract) 37 (1992) 1409.

DeBoo, J.C., R.E. Waltz, T.H. Osborne, "Comparison of Dimensionally Similar Discharges with Similar Heat Deposition Profiles," in Proc. 18th European Conference on Controlled Fusion and Plasma Physics, Berlin, 1991, (European Physical Society, PetitLancy, Switzerland, 1991) Vol. 15C, Part I p. 173; General Atomics Report GA-A20466 (1991).

deGrassie, J.S., R.I. Pinsker, C.C. Petty, Y.R. Lin-Liu, et al., "Fast Wave Heating and Current Drive System Upgrade for DIII-D," in Bull. 34th Annual American Physical Society Meeting, November 16-20, 1992, Seattle, Washington, (Abstract) 37 (1992) 1513.

deGrassie, J.S., F.W. Baity, R.C. Callis, D.J. Hoffman, et al., "Fast Wave Current Drive System Design for DIII-D," to be published in Proc. 17th Symp. on Fusion Technology, September 14-18, 1992, Rome, Italy; General Atomics Report GA-A21043 (1992).

DIII-D Team, "Recent DIII-D Results," in Proc. 14th International Conference on Plasma Physics and Controlled Nuclear Fusion Research, September 30 through October 7, 1992, Wurzburg, Germany; General Atomics Report GA-A21089 (1992).

Doane, J.L., "Grating Polarizers in Waveguide Miter Bands," International Journal of Infrared and Millimeter Waves 13 (1992) 1727; General Atomics Report GA-A20892 (1992).

Doyle, E.J., K.W. Kim, W.A. Peebles, C.L. Rettig, and T.L. Rhodes, "Investigation of In/Out Turbulence Asymmetries on DIII-D," in Bull. 34th Annual American Physical Society Meeting, November 16-20, 1992, Seattle, Washington, (Abstract) 37 (1992) 1510. 
Duong, H.H., W.W. Heidbrink, C.T. Hsu, R.L. Lee, et al., "Loss of Beam Ions During TAE Instabilities," in Bull. 34th Annual American Physical Society Meeting, November 16-20, 1992, Seattle, Washington, (Abstract) 37 (1992).

Duong, H.H., and W.W. Heidbrink, "Confinement of Fusion-Produced MeV Ions in the DIII-D Tokamak," submitted to Nucl. Fusion; General Atomics Report GA-A20923 (1992).

Evans, T.E., "Statistical Properties of Intrinsic Topological Noise in Tokamaks," in Proc. 18th European Conference on Controlled Fusion and Plasma Physics, Berlin, 1991, (European Physical Society, Petit-Lancy, Switzerland, 1991) Vol. 15C, Part II, p. 65; General Atomics Report GA-A20448 (1991).

Evans, T.E., D.N. Hill, T.W. Petrie, A.W. Leonard, et al., "Radial Heat Flux Profile Bifurcation During High Power DIII-D Single- and Double-Null Divertor Operations," in Bull. 34th Annual American Physical Society Meeting, November 16-20, 1992, Seattle, Washington, (Abstract) 37 (1992) 1565.

Ferron, J.R., "A High Speed Data Acquisition and Processing System for Real Time Data Analysis and Control," Rev. Sci. Instrum. 63 (1992) 5464; General Atomics Report GA-A20810 (1992).

Ferron, J.R., A. Kellman, E. McKee, T.H. Osborne, et al., "An Advanced Plasma Control System for the DIII-D Tokamak," in Proc. 14th Symp. on Fusion Engineering, IEEE (San Diego, California, September 30-October 3, 1991) Vol. II (1992) 761; General Atomics Report GA-A20641 (1991).

Ferron, J.R., and E.J. Strait, "Real Time Analysis of Tokamak Discharge Parameters," Rev. Sci. Instrum. 63 (1992) 4799; General Atomics Report GA-A20860 (1992).

Ferron, J.R., L.L. Lao, and T.S. Taylor, "The Effect of Current Profile Changes on Confinement in the DIII-D Tokamak," in Proc. 19th European Conference on Controlled Fusion and Plasma Heating, Innsbruck, Austria, 1992, (European Physical Society, Petit-Lancy, Switzerland, 1991) Vol. 16C, Part I, p. 55; General Atomics Report GA-A20914 (1992).

Ferron, J.R., L.L. Lao, T.S. Taylor, Y.B. Kim, et al., "Improved Confinement and Stability in the DIII-D Tokamak Obtained Through Modification of the Current Profile," to be published in a special issue of Phys. Fluids B (1993); General Atomics Report GA-A21151 (1992). 
Finkenthal, D.F., D.L. Hillis, M.R. Wade, J.T. Hogan, et al., "Instrumentation and Calibration of the DIII-D Charge Exchange Recombination Spectroscopy System to Measure Absolute Helium Density Profiles," in Bull. 34th Annual American Physical Society Meeting, November 16-20, 1992, Seattle, Washington, (Abstract) 37 (1992) 1568.

Finkenthal, D.F., D.L. Hillis, M.R. Wade, W.P. West, et al., "Helium Transport Studies on DIII-D," in Proc. 19th European Conference on Controlled Fusion and Plasma Heating, Innsbruck, Austria, 1992, (European Physical Society, Petit-Lancy, Switzerland, 1991) Vol. 16C, Part I, p. 275; General Atomics Report GA-A20933 (1992).

Futch, A.H., J.W. Cuthbertson, D. Buchenauer, D.N. Hill, et al., "Heat Transmission at the Divertor Sheath of DIII-D," in Bull. 34th Annual American Physical Society Meeting, November 16-20, 1992, Seattle, Washington, (Abstract) 37 (1992) 1567.

Gohil, P., K.H. Burrell, E.J. Doyle, R.J. Groebner, et al., "Study of Edge Electric Field and Edge Microturbulence at the L-H Transition in DIII-D," in Proc. 18th EPS Conf on Controlled Fusion and Plasma Physics (June 3-7, 1991, Berlin, Germany) Vol. 15C, Part I (1991) 289; General Atomics Report GA-A20481 (1991).

Gohil, P., K.H. Burrell, R.J. Groebner, J. Kim, et al., "The Charge Exchange Recombination Diagnostic System on the DIII-D Tokarnak," in Proc. 14th Symp. on Fusion Engineering, IEEE (San Diego, California, September 30-October 3, 1991) Vol. II (1992) 1199; General Atomics Report GA-A20652 (1991).

Greene, K.L., "Displaying DIII-D Plasma Data Using DEC's X Window System," in Proc. 14th Symp. on Fusion Engineering, IEEE (San Diego, California; September 30-October 3, 1991) Vol. II (1992) 773; General Atomics Report GA-A20610 (1991).

Greenfield, C.M., G.L. Jackson, K.H. Burrell, J.C. DeBoo, et al., "VH-Mode Discharges in the DIII-D Tokamak," in Proc. 19th European Conference on Controlled Fusion and Plasma Heating, Innsbruck, Austria, 1992, (European Physical Society, Petit-Lancy, Switzerland, 1991) Vol. 16C, Part I, p. 11; General Atomics Report GA-20917 (1992).

Groebner, R.J., K.H. Burrell, P. Gohil, J. Kim, and R.P. Seraydarian, "Edge Ion Dynamics in H-Mode Discharges in DIII-D," in Proc. 19th European Conference on Controlled Fusion and Plasma Heating, Innsbruck, Austria, 1992, (European Physical Society, PetitLancy, Switzerland, 1991) Vol. 16C, Part I, p. 183; General Atomics Report GA-A20912 (1992). 
Groebner, R.J., "An Emerging Understanding of H-Mode Discharges in Tokamaks," in Bull. 34th Annual American Physical Society Meeting, November 16-20, 1992, Seattle, Washington, (Abstract) 37 (1992) 1352.

Groebner, R.J., "An Emerging Understanding of H-Mode Discharges in Tokamaks," to be published in special issue of Phys. Fluids B (1993); General Atomics Report GA-A21128 (1992).

Harris, T.E., "Active Heater Control and Regulation for the Varian VGT-8011 Gyrotron," in Proc. 14th Symp. on Fusion Engineering, IEEE (San Diego, California, September 30-October 3, 1991) Vol. I (1992) 130; General Atomics Report GA-A20645 (1991).

Harvey, R.W., S.C. Chiu, M.G. McCoy, G.D. Kerbel, et al., “3D Fokker-Planck Calculation of Combined Fast Wave/Lower Hybrid and Electron Cyclotron Current Drive in Tokamaks," in Proc. IAEA Technical Committee Meeting on Fast Wave Current Drive Reactor Scale Tokamaks, September 23-25, 1991, Arles, France; General Atomics Report GA-A20752 (1992).

Heidbrink, W.W., H.H. Duong, R.J. La Haye, E.J. Strait, and A.D. Turnbull, "Characteristics of Beam-Driven Instabilities," in Bull. 34th Annual American Physical Society Meeting, November 16-20, 1992, Seattle, Washington, (Abstract) 37 (1992) 1511.

Hill, D.N., A.H. Futch, A.W. Leonard, M.A. Mahdavi, et al., "The Effect of ELMs on Edge Plasma Scaling in DIII-D, J. of Nucl. Materials 196-198 (1992) 204; General Atomics Report GA-A20962 (1992).

Hill, D.N., R.B. Campbell, J.W. Cuthbertson, R.J. Kanzieiter, et al., "Variation of Divertor Plasma Parameters With X-Point to Target-Plate Distance," in Bull. 34th Annual American Physical Society Meeting, November 16-20, 1992, Seattle, Washington, (Abstract) 37 (1992) 1565.

Hillis, D.L., J.T. Hogan, K.H. Finken, W.P. West, et al., "Helium Transport in Enhanced Confinement Regimes on the TEXTOR and DIII-D Tokamaks," to be published in Proc. 14th International Conference on Plasma Physics and Controlled Nuclear Fusion Research, September 30 through October 7, 1992, Wurzburg, Germany; General Atomics Report GA-A20901 (1992).

Hodapp, T.R., G.L. Jackson, J. Phillips, K.L. Holtrop, et al., "A System to Deposit Boron Films (Boronization) in the DIII-D Tokamak," in Proc. 14th Symp. on Fusion Engineering, IEEE (San Diego, California, September 30-October 3, 1991) Vol. I (1992) 392; General Atomics Report GA-A20624 (1991). 
Holtrop, K.L., G.L. Jackson, K.M. Schaubel, A.G. Kellman, "Glow Discharge Initiation with Electron Gun Assist," in Proc. 14th Symp. on Fusion Engineering, IEEE (San Diego, California, September 30-October 3, 1991) Vol. II (1992) 749; General Atomics Report GA-A20617 (1991).

Holtrop, K., A.G. Kellman, and A. Astapkovich, "Modeling of Current Decay Rates During Disruptions in DIII-D," in Bull. 34th Annual American Physical Society Meeting, November 16-20, 1992, Seattle, Washington, (Abstract) 37 (1992) 1570.

Hong, R., A.P. Colleraine, D.H. Kellman, J. Kim, et al., "Enhancement of DIII-D Neutral Beam System for Higher Performance," to be published in Proc. 17th Symp. on Fusion Technology, September 14-18, 1992, Rome, Italy; General Atomics Report GA-A21034 (1992).

Howl, W., A.D. Turnbull, T.S. Taylor, L.L. Lao, et al., "Sensitivity of the Kink Instability of the Pressure Profile," Phys. Fluids B 4 (1992) 1724; General Atomics Report GA-A19953 (1991).

Hsieh, C.L., R.E. Stockdale, M.S. Chu, J.C. DeBoo, et al., "Experimental Constraints on Electron Thermal Conductivity in Tokamaks," in Bull. 34th Annual American Physical Society Meeting, November 16-20, 1992, Seattle, Washington, (Abstract) 37 (1992) 1509.

Humphreys, D.A., and A.G. Kellman, "Modeling and Analysis of Plasma Motion During Disruption in DIII-D," in Bull. 34th Annual American Physical Society Meeting, November 16-20, 1992, Seattle, Washington, (Abstract) 37 (1992) 1570.

Hyatt, A.W., R.J. La Haye, T.H. Jensen, R.J. Groebner, and J. T. Scoville, “Applied Magnetic Island Braking Forces in DIII-D," in Bull. 34th Annual American Physical Society Meeting, November 16-20, 1992, Seattle, Washington, (Abstract) 37 (1992) 1569.

Ikezi, H., R.I. Pinsker, and Y.R. Lin-Liu, "Fast Wave Propagation in DIII-D," in Bull. 34th Annual American Physical Society Meeting, November 16-20, 1992, Seattle, Washington, (Abstract) 37 (1992) 1513.

Jackson, G.L., J. Winters, T.S. Taylor, K.H. Burrell, et al., "VH-Mode in DIII-D After Boronization," Phys. Fluids B 4 (1992) 2181; General Atomics Report GA-A20787 (1992).

Jackson, G.L., K.H. Burrell, J.C. DeBoo, C.M. Greenfield, et al., "Boronization and VH-Mode Discharges," in Bull. 34th Annual American Physical Society Meeting, November 16-20, 1992, Seattle, Washington, (Abstract) 37 (1992) 1509. 
Jackson, G.L., J. Winters, K.H. Burrell, J.C. DeBoo, et al., "Boronization in DIII-D," in J. Nucl. Mater. 196-198 (1992) 236; General Atomics Report GA-A20904 (1992).

James, R.A., R.W. Harvey, Y.R. Lin-Liu, M.G. McCoys, "Experimental and Theoretical Analysis of DIII-D Electron Cyclotron Current Drive Experiments in a Non-Linear Regime," in Bull. 34th Annual American Physical Society Meeting, November 16-20, 1992, Seattle, Washington, (Abstract) 37 (1992) 1513.

Jensen, T.H., A.W. Leonard, A.W. Hyatt, "A Comprehensive Model for Driven Islands in Tokamaks," in Bull. 34th Annual American Physical Society Meeting, November 16-20, 1992, Seattle, Washington, (Abstract) 37 (1992) 1568.

Jensen, T.H., J.M. Greene, P.A. Politzer, "Large Anomaly of the Perpendicular Resistivity of a Tokamak," in Bull. 33rd Annual American Physical Society Meeting, November 4-8, 1991, Tampa, Florida (Abstract) 36 (1991) 2412; General Atomics Report GA-A20557 (1991); Phys. Fluids B 4 (1992) 760.

Jong, R.A., D.N. Hill, and G.D. Porter, "Comparison of Divertor Models with Divertor Heat Flux Measuements in DIII-D," in Bull. 34th Annual American Physical Society Meeting, November 16-20, 1992, Seattle, Washington, (Abstract) 37 (1992) 1566.

Kamada, Y., R.T. Snider, T.S. Taylor, E.J. Strait, and J.R. Ferron, "Sawtooth Frequency Studies in DIII-D Tokamak," to be published in Nucl. Fusion (1992).

Kawashima, H., V.M. Trukhin, J. Lohr, R.W. Harvey, et al., "X-Ray Energy Analysis for Radio Frequency Current Drive Experiments in the DIII-D Tokamak," General Atomics Report GA-A20957 (1992).

Kessler, D., "Power Distribution Within the Accelerator Column of a DIII-D Neutral Beam Source," in Bull. 34th Annual American Physical Society Meeting, November 16-20, 1992, Seattle, Washington, (Abstract) 37 (1992) 1496.

Khayrutdinov, R.R., V.E. Lukash, A.G. Kellman, and D.A. Humphreys, "DINA Disruption Simulation of DIII-D Discharges," in Bull. 34th Annual American Physical Society Meeting, November 16-20, 1992, Seattle, Washington, (Abstract) 37 (1992) 1570.

Kim, J., K.H. Burrell, P. Gohil, R.J. Groebner, et al., "Comparison of Measured Ion Poloidal Flow Velocities With Predictions of Neoclassical Theory," in Bull. 34th Annual American Physical Society Meeting, November 16-20, 1992, Seattle, Washington, (Abstract) 37 (1992) 1510. 
Klepper, C.C., J.T. Hogan, L.W. Owen, P.K. Mioduszewski, et al., "Comparison of Transient and Stationary Neutral Pressure Response in the DIII-D Advanced Divertor," J. Nucl. Mater. 196-198 (1992) 1090; General Atomics Report GA-A20900 (1992).

Klepper, C.C., J.T. Hogan, D.N. Hill, R. Maingi, et al., "Divertor Neutral Pressure Enhancement With a Baffle in DIII-D," to be published in Nucl. Fusion (1993); General Atomics Report GA-A20774 (1992).

Konoshima, S., T.H. Osborne, K.H. Burrell, M.S. Chu, et al., "The Effect of Plasma Shaping 'Triangularity' on VH-Mode Confinement in the DIII-D Tokamak," in Bull. 34th Annual American Physical Society Meeting, November 16-20, 1992, Seattle, Washington, (Abstract) 37 (1992) 1509.

Kurki-Suonio, T., and K.H. Burrell, "Investigation of Electron-Ion Temperature Differences at the Edge of H-Mode Plasmas in DIII-D," in Bull. 34th Annual American Physical Society Meeting, November 16-20, 1992, Seattle, Washington, (Abstract) 37 (1992) 1510.

Kurki-Suonio, T., K.H. Burrell, R.J. Groebner, R. Philipona, and C.L. Rettig, "Transport and Fluctuations in the Interior of the DIII-D Plasmas," to be published in Nucl. Fusion (1993); General Atomics Report GA-A21017 (1992).

Kurki-Suonio, T., R.J. Groebner, K.H. Burrell, "Changes in Local Confinement After an L-H Transition in DIII-D," Nucl. Fusion 32 (1992) 133; General Atomics Report GA-A20450 (1991).

La Haye, R.J., "Error Field Considerations for BPX," in Proc. 14th Symp. on Fusion Engineering, IEEE (San Diego, California, September 30-October 3, 1991) Vol. II (1992) 1023; General Atomics Report GA-A20579 (1991).

La Haye, R.J., R.U. Fitzpatrick, T.C. Hender, et al., "Critical Error Fields for Locked Mode Instability in Tokamaks," Phys. Fluids B 4 (1992) 2098; General Atomics Report GA-A20709 (1991).

La Haye, R.J., R. Fitzpatrick, T.C. Hender, A.W. Morris, et al., "Critical Error Fields for Locked Mode Instability in Tokamaks," Phys. Fluids B 4 (1992) 2098; General Atomics Report GA-A20709 (1992).

La Haye, R.J., R.J. Groebner, A.W. Hyatt, C.L. Rettig, and J.T. Scoville, "Effect of Magnetic Braking of Plasma Rotation on the H-Mode Radial Electric Field, Confinement and Microturbulence in DIII-D," in Bull. 34th Annual American Physical Society Meeting, November 16-20, 1992, Seattle, Washingto, (Abstract) 37 (1992) 1568. 
La Haye, R.J., R.J. Groebner, A.W. Hyatt, and J.T. Scoville, "Effect of Magnetic Braking of the Plasma Rotation on the H-Mode Radial Electric Field and Energy Confinement in the DIII-D Tokamak," to be published in Nucl. Fusion (1993); General Atomics Report GA-A20782 (1992).

La Haye, R.J., A.W. Hyatt, and J.T. Scoville, "Non-Linear Instability to Low $\mathrm{m}, \mathrm{n}=1$ Error Fields in DIII-D as a Function of Plasma Fluid Rotation and Beta," Nucl. Fusion 32 (1992) 2119; General Atomics Report GA-A20824 (1992).

La Haye, R.J., R.J. Groebner, A.W. Hyatt, and J.T. Scoville, "Investigation of the Effect of Large Core Changes in Toroidal Plasma Rotation and Radial Electric Field on Confinement in H-Mode Discharges in the DIII-D Tokamak," in Proc. 19th European Conference on Controlled Fusion and Plasma Heating, Innsbruck, Austria, 1992, (European Physical Society, Petit-Lancy, Switzerland, 1991) Vol. 16C, Part I, p. 187; General Atomics Report GA-A20909 (1992).

La Haye, R.J., R.J. Groebner, A.W. Hyatt, C.L. Rettig, and J.T. Scoville, "Effect of Magnetic Braking of Plasma Rotation on the H-Mode Radial Electric Field, Confinement and Microturbulence in DIII-D," in Bull. 34th Annual American Physical Society Meeting, November 16-20, 1992, Seattle, Washington, (Abstract) 37 (1992) 1568.

Lao, L.L., J.R. Ferron, T.S. Taylor, V.S. Chan, et al., "Regimes of Improved Confinement and Stability in DIII-D Obtained Through Current Profile Modifications," to be published in Proc. 14th International Conference on Plasma Physics and Controlled Nuclear Fusion Research, September 30 through October 7, 1992, Wurzburg, Germany; General Atomics Report GA-A21053 (1992).

Lao, L.L., V.S. Chan, M.S. Chu, J.R. Ferron, et al., "High Li Improved Confinement H-Mode Discharges Obtained by Dynamic Shaping Technique in the DIII-D Tokamak," in Bull. 34th Annual American Physical Society Meeting, November 16-20, 1992, Seattle, Washington, (Abstract) 37 (1992) 1509.

Lao, L.L., T.S. Taylor, M.S. Chu, A.D. Turnbull, et al., "Effects of Current Profiles on MHD Stability," in Proc. 18th European Conference on Controlled Fusion and Plasma Heating, Berlin, Germany, 1991, (European Physical Society, Petit-Lancy, Switzerland, 1991) Vol. 15C, Part IV, p. 73; General Atomics Report GA-A20479 (1991).

Lazarus, E.A., L.L. Lao, T.H. Osborne, T.S. Taylor, et al., "An Optimization of Beta in the DIII-D Tokamak," Phys. Fluids B 4 (1992) 3644; General Atomics Report GA-A20571 (1991). 
Lazarus, E.A., L.L. Lao, E.J. Strait, and T.H. Osborne, "b.t Versus Plasma Shape in DIII-D," in Bull. 34th Annual American Physical Society Meeting, November 16-20, 1992, Seattle, Washington, (Abstract) 37 (1992) 1410.

Lee, R.L., A.G. Kellman, D.N. Hill, T.W. Petrie, and R. Ellis, "Heat Flux Deposition During Disruptions in DIII-D," in Bull. 34th Annual American Physical Society Meeting, November 16-20, 1992, Seattle, Washington, (Abstract) 37 (1992).

Leonard, A.W., D.N. Hill, T.W. Petrie, G.D. Porter, and R.A. Jong, "Power Balance and Divertor Performance in DIII-D," in Bull. 34th Annual American Physical Society Meeting, November 16-20, 1992, Seattle, Washington, (Abstract) 37 (1992) 1565.

Lin-Liu, Y.R., R.W. Harvey, R.A. James, and M.G. McCoy, "Modeling of DIII-D Electron Cyclotron Current Drive Using CQL3D," in Bull. 34th Annual American Physical Society Meeting, November 16-20, 1992, Seattle, Washington, (Abstract) 37 (1992) 13.

Lippmann, S.I., T.E. Evans, G.L. Jackson, and W.P. West, "Impurity Penetration and Transport During VH-Mode on DIII-D," J. Nucl. Mater. 196-198 (1992) 498; General Atomics Report GA-A20903 (1992).

Lippmann, S.I., T.E. Evans, F. Allais, C. DeMichelis, et al., "Measurement of Poloidal Asymmetries in Tore Supra During Ergodic Divertor Operations," in Bull. 34th Annual American Physical Society Meeting, November 16-20, 1992, Seattle, Washington, (Abstract) 37 (1992) 1449.

Lohr, John, R.W. Harvey, R.A. James, T.C. Luce, et al., "Analysis of NonInductive Current Drive from ECCD and Bootstrap on T-10," in Proc. 9th Topical Conf. on Radio Frequency (August 19-21, 1991, Charleston, South Carolina) Vol. 144, p. 255; General Atomics Report GA-A20647 (1991).

Luce, T.C., and C.C. Petty, "Modeling of Off-Axis Heating Discharges," in Bull. 34th Annual American Physical Society Meeting, November 16-20, 1992, Seattle, Washington, (Abstract) 37 (1992) 1410.

Luce, T.C., C.C. Petty, R. Prater, R.W. Harvey, et al., "Recent Results From the $60 \mathrm{GHz}$ Inside Launch ECH System on the DIII-D Tokamak," to be published in Proc. 8th Joint Workshop on Electron Cyclotron Emission and Electron Cyclotron Resonance Heating, October 18-21, 1992, Garching, Federal Republic of Germany; General Atomics Report GA-A21134 (1992).

Mahdavi, M.A., S.L. Allen, T.E. Evans, D.N. Hill, et al., "Applications of Divertor Biasing to the Next Generation Tokamaks," General Atomics Report GAA21064 (1992). 
Mahdavi, M.A., "Divertor Biasing Experiments on the DIII-D Tokamak," in Bull. 34th Annual American Physical Society Meeting, November 16-20, 1992, Seattle, Washington, (Abstract) 37 (1992) 1526.

Makowski, M.A., T.C. Luce, C. Forest, C.C. Petty, et al., "Analysis of ECH Modulated Discharges in DIII-D," in Bull. 34th Annual American Physical Society Meeting, November 16-20, 1992, Seattle, Washington, (Abstract) 37 (1992) 1511.

Matsumoto, H., K.H. Burrell, T.N. Carlstrom, et al., "Suppression of the Edge Turbulence at the L to H Transition in DIII-D," Plasma Phys. and Contr. Fusion 34 (1992) 615; General Atomics Report GA-A20383 (1991).

Matthews, G., D.N. Buchenauer, D.N. Hill, M.A. Mahdavi, et al., "Impurity Transport at the DIII-D Divertor Strike Points," in Proc. 18th European Conference on Controlled Fusion and Plasma Heating, Berlin, Germany, 1991, (European Physical Society, PetitLancy, Switzerland, 1991) Vol. 15C, Part III, p. 229; General Atomics Report GA-A20472 (1991).

Mayberry, M.J., R.I. Pinsker, S.C. Chiu, G.L. Jackson, et al., "Fast Wave Current Drive Antenna Performance on DIII-D," in Proc. 9th Topical Conf. on Radio Frequency Power in Plasmas, AIP (August 19-21, 1991, Charleston, South Carolina) (1992) p. 276; General Atomics Report GA-A20694 (1991).

Mayberry, M.J., R.I. Pinsker, C.C. Petty, M. Porkolab, et al., "Ion Bernstein Wave Antenna Loading Measurements on the DIII-D Tokamak," to be published in Nucl. Fusion (1993); General Atomics Report GA-A20660 (1991).

McHarg, Jr., B.B., "The Use of a VAX Cluster for the DIII-D Data Acquisition System," in Proc. 14th Symp. on Fusion Engineering, IEEE (San Diego, California, September 30-October 3, 1991) Vol. II (1992) 852; General Atomics Report GA-A20584 (1991).

Menon, M.M., M.J. Schaffer, and A.G. Kellman, "Voltage Transients Induced by Plasma Disruptions in the DIII-D Tokamak," in Bull. 34th Annual American Physical Society Meeting, November 16-20, 1992, Seattle, Washington, (Abstract) 37 (1992) 1570.

Moeller, C., R. Callis, W. DeHope, J. Doane, et al., "110 GHz ECH System for DIII-D," in Proc. 18th European Conf. on Controlled Fusion and Plasma Physics (June 3-7, 1991, Berlin, Germany) Vol. 15C, Part III (1991) 369; General Atomics Report GA-A20471 (1991). 
Moeller, C.P., J.L. Doane, "A Coaxial Converter for Transforming a Whispering Gallery Mode to the Hell Mode," in Proc. 9th Topical Conf on Radio Frequency (August 19-21, 1991, Charleston, South Carolina) Vol. 244 (1992) 326; General Atomics Report GA-A20628 (1991).

Moyer, R.A., J.G. Watkins, K.H. Burrell, R.W. Conn, et al., "Turbulence and Particle Transport Measurements in the DIII-D Boundary With the Fast Reciprocating Langmuir Probe," in Bull. 34th Annual American Physical Society Meeting, November 16-20, 1992, Seattle, Washington, (Abstract) 37 (1992) 1411.

Parks, P.B., "Electric Field and Current Distribution Near the Ablation Cloud of a Pellet Injected into a Tokamak," Nucl. Fusion 32 (1992) 2137; General Atomics Report GA-A20761 (1992).

Peebles, W.A., E.J. Doyle, C.L. Rettig, T.L. Rhodes, et al., "Edge and Interior Turbulence Measurements on DIII-D Via reflectometry and Collective Scattering," in Bull. 34th Annual American Physical Society Meeting, November 16-20, 1992, Seattle, Washington, (Abstract) 37 (1992) 1512.

Petersen, P.I., S.M. Miller, "The DIII-D Tokamak Trouble Report Database," in Proc. 14th Symp. on Fusion Engineering, IEEE (San Diego, California, September 30-October 3, 1991) Vol. II (1992) 776; General Atomics Report GA-A20612 (1991).

Petrie, T.W., D. Buchenauer, D.N. Hill, C.C. Klepper, et al., "Divertor Heat Flux Reduction by D2 Injection in DIII-D, J. Nucl. Mater. 196-198 (1992) 848; General Atomics Report GA-A20899 (1992).

Petrie, T.W., D. Buchenauer, R. Campbell, J.W. Cuthbertson, et al., "Divertor Heat Flux Reduction by D2 Injection in DIII-D," in Bull. 34th Annual American Physical Society Meeting, November 16-20, 1992, Seattle, Washington, (Abstract) 37 (1992) 1565.

Petrie, T.W., D.N. Hill, D. Buchenauer, A. Futch, et al., "Recent Gaseous Divertor Experiments in DIII-D," in Proc. 18th European Conference on Controlled Fusion and Plasma Heating, Berlin, Germany, 1991, (European Physical Society, Petit-Lancy, Switzerland, 1991) Vol. 15C, Part III, p. 237; General Atomics Report GA-A20478 (1991).

Petty, C.C., T.C. Luce, J.C.M. deHaas, R.A. James, et al., "Non-Diffusive Heat Iransport During Electron Cyclotron Heating on the DIII-D Tokamak," in Proc. 18th European Conference on Controlled Fusion and Plasma Heating, Berlin, Germany, 1991, (European Physical Society, Petit-Lancy, Switzerland, 1991) Vol. 15C, Part I, p. 241; General Atomics Report GA-A20465 (1991). 
Petty, C.C., R.I. Pinsker, M.J. Mayberry, S.C. Chiu, et al., "Direct Electron Heating by 60 MHz Fast Waves on DIII-D," in Proc. 9th Topical Conf. on Radio Frequency (August 19-21, 1991, Charleston, South Carolina) (1992) p. 96; General Atomics Report GA-A20688 (1991).

Petty, C.C., R.I. Pinsker, M.J. Mayberry, M. Porkolab, et al., "Fundamental and Second Harmonic Hydrogen Fast-Wave Heating on DIII-D," in Proc. 9th Topical Conf. on Radio Frequency (August 19-21, 1991, Charleston, South Carolina) (1992) p. 133; General Atomics Report GA-A20693 (1991).

Petty, C.C., R.I. Pinsker, M. Porkolab, F.W. Baity, et al., "Direct Electron Absorption of Fast Waves on the DIII-D Tokamak," Phys. Rev. Lett. 69 (1992) 289; General Atomics Report GA-A20934 (1992).

Petty, C.C., R.I. Pinsker, M. Porkolab, F.W. Baity, et al., "Fast Wave Current Drive Experiments on the DIII-D Tokamak," in Proc. 19th European Conference on Controlled Fusion and Plasma Heating, June 29 through July 3, 1992, Innsbruck, Austria, Vol. 16C, Part II (1992) 893; General Atomics Report GA-A20930 (1992).

Petty, C.C., R.I. Pinsker, M.J. Mayberry, M. Porkolab, et al., "Absorption of Fast Waves by Electrons on the DIII-D Tokamak," Phys. Rev. Lett. 69 (1992) 289; General Atomics Report GA-A20777 (1992).

Petty, C.C., R.I. Pinsker, M. Porkolab, F.W. Baity, et al., "Fast Wave Current Drive Experiments in DIII-D," in Bull. 34th Annual American Physical Society Meeting, November 16-20, 1992, Seattle, Washington, (Abstract) 37 (1992) 1411.

Phelps, D.A., C.P. Moeller, R.W. Gould, and S.C. Chiu, "Modeling of Plasma Loading and Unidirectional Coupling to Fast Waves with a New Broadband Traveling Wave Antenna: The Combline Structure," in Bull. 34th Annual American Physical Society Meeting, November 16-20, 1992, Seattle, Washington, (Abstract) 37 (1992) 1513.

Phillips, J., T. Hodapp, K. Holtrop, G.L. Jackson, and J. Winters, "Initial Boronization of the DIII-D Tokamak," J. Vac. Science and Technol. A 10 (1992) 1252; General Atomics Report GA-A20750 (1991).

Philipona, R., R.J. Groebner, K.H. Burrell, E.J. Doyle, et al., "Two-Stage Turbulence Suppression and E x B Plasma Flow Measured at the L-H Transition," Phys. Fluids B 5 (1993) 87; General Atomics Report GA-A20516 (1991). 
Pinsker, R.I., M.J. Mayberry, C.C. Petty, W.P. Cary, et al., "30-60 MHz FWCD System on DIII-D: Power Division, Phase Control, and Tuning for a Four-Element Antenna Array," in Proc. 14th Symp. on Fusion Engineering, IEEE (San Diego, California, September 30-October 3, 1991) Vol. I (1992) 115; General Atomics Report GA-A20619 (1991).

Pinsker, R.I., M.J. Mayberry, C.C. Petty, M. Porkolab, et al., "ICRF Heating Experiments on DIII-D," in Proc. 9th Topical Conf. on Radio Frequency (August 19-21, 1991, Charleston, South Carolina) (1992) p. 105; General Atomics Report GA-A20697 (1991).

Pinsker, R.I., C.C. Petty, M. Porkolab, R. Prater, et al., "Results From Faraday-Shieldless Operation of the DIII-D Fast Wave Current Drive Antenna," in Bull. 34th Annual American Physical Society Meeting, November 16-20, 1992, Seattle, Washington, (Abstract) 37 (1992) 1512.

Politzer, P.A., and V.S. Chan, "RF-Enhanced Bootstrap Current," in Bull. 34th Annual American Physical Society Meeting, November 16-20, 1992, Seattle, Washington, (Abstract) 37 (1992) 1514.

Prater, R., T.C. Luce, and C.C. Petty, "Transport Analysis of Off-Axis Heating Discharges," in Bull. 34th Annual American Physical Society Meeting, November 16-20, 1992, Seattle, Washington, (Abstract) 37 (1992) 1511.

Prater, R., R.A. James, C.C. Petty, R.I. Pinsker, et al., "Current Drive With Fast Waves, Electron Cyclotron Waves, and Neutral Injection in the DIII-D Tokamak," to be published in Plasma Physics and Controlled Fusion; General Atomics Report GA-A21003 (1992).

Project Staff, T.C. Simonen, T.E. Evans (editors), "DIII-D Research Operations Annual Report to the U.S. Department of Energy," General Atomics Report GA-A20790 (1992).

Reis, E., I. Almajan, C.B. Baxi, M.M. Menon, et al., "Design and Analysis of the Cryopump for the DIII-D Advanced Divertor," in Proc. 17th Symp. on Fusion Technology, September 14-18, 1992, Rome, Italy; General Atomics Report GA-A21016 (1992).

Reis, E., R.D. Blevins, T.H. Jensen, J.L. Luxon, et al., "Modeling and Measurement of the Motion of the DIII-D Vacuum Vessel During Vertical Instabilities," in Proc. 14th Symp. on Fusion Engineering, IEEE (San Diego, California, September 3-October 3,1991) Vol. I (1992) 37; General Atomics Report GA-A20636 (1991). 
GA-A21186; FY92 DIII-D Research Operations Annual Report

Rensink, M.E., S.L. Allen, A.H. Futch, D.N. Hill, et al., "Particle Transport Studies for Single-Null Divertor Discharges in DIII-D," to be published in Phys. Fluids B (1993); General Atomics Report GA-A20510 (1992).

Rettig, C.L., E.J. Doyle, N.C. Luhmann, Jr., W.A. Peebles, et al., "Microturbulence Drive/Suppression Mechanisms in DIII-D," in Bull. 34th Annual American Physical Society Meeting, November 16-20, 1992, Seattle, Washington, (Abstract) 37 (1992) 1526.

Riggs, S., "Recent Measurements of Divergence and Species Mix for DIII-D Neutral Beams With a New Doppler Shifted Spectroscopy Analysis Routine," in Bull. 34th Annual American Physical Society Meeting, November 16-20, 1992, Seattle, Washington, (Abstract) 37 (1992) 1496.

Schaffer, M.J., S.I. Lippmann, M.A. Mahdavi, T.W. Petrie, et al., "Particle Control in the DIII-D Advanced Divertor," in Proc. 14th Symp. on Fusion Engineering, IEEE (San Diego, California, September 30-October 3,1991) Vol. I (1992) 197; General Atomics Report GA-A20631 (1991).

Schaffer, M.J., M.A. Mahdavi, C.C. Klepper, D.N. Hill, M.E. Rensink, "Effect of Divertor Bias on Plasma Flow in the Tokamak Scrape-off Layer," Nucl. Fusion 32 (1991) 855; General Atomics Report GA-A20474 (1991).

Schaffer, M.J., S. Yamaguchi, and Y. Kondoh, "Preliminary Oscillating Fluxes Current Drive Experiment in DIII-D Tokamak," submitted to Nucl. Fusion (1993); General Atomics Report GA-A20613 (1992).

Schaffer, M.J., S.L. Allen, N.H. Brooks, D. Buchenauer, et al., "Advanced Divertor Program Results from DIII-D," to be published in Proc. 14th International Conference on Plasma Physics and Controlled Nuclear Fusion Research, September 30 through October 7 , 1992, Wurzburg, Germany; General Atomics Report GA-A21044 (1992).

Schaffer, M.J., D. Buchenauer, J.W. Cuthbertson, R. Ellis, et al., "Edge Effects During DIII-D Divertor Biasing," General Atomics Report GA-A21060 (1992).

Schaffer, M.J., T.R. Jarboe, A.W. Hyatt, M.A. Mahdavi, et al., "Non-Inductive Startup Experiments on DIII-D," in Bull. 34th Annual American Physical Society Meeting, November 16-20, 1992, Seattle, Washington, (Abstract) 37 (1992) 1566. 
Schaubel, K.M., C.B. Baxi, G.L. Campbell, A.M. Gootgeld, et al., "Design of the Advanced Divertor Pump Cryogenic System for DIII-D," in Proc. 14th Symp. on Fusion Engineering, IEEE, San Diego, California, September 30-October 3, 1991) Vol. II (1992) 1222; General Atomics Report GA-A20607 (1991).

Schissel, D.P., T.H. Osborne, T.N. Carlstrom, and H. Zohm, "A Quantitative Analysis of the Effect of ELMs on H-Mode Thermal Energy Confinement in DIII-D," in Proc. 19th European Conference on Controlled Fusion and Plasma Heating, Innsbruck, Austria, 1992, (European Physical Society, Petit-Lancy, Switzerland, 1991) Vol. 16C, Part I, p. 235; General Atomics Report GA-A20942 (1992).

Schissel, D.P., T.H. Osborne, J.C. DeBoo, J.R. Ferron, et al., “An Examination of Heat Transport During Off Axis Neutral Beam Injection in DIII-D," Nucl. Fusion 32 (1992) 689; General Atomics Report GA-A20696 (1991).

Schissel, D.P., T.H. Osborne, J.C. DeBoo, J.R. Ferron, and R.E. Waltz, "Heat Transport During Off-Axis NBI in DIII-D," in Bull. 34th Annual American Physical Society Meeting, November 16-20, 1992, Seattle, Washington, (Abstract) 37 (1992) 1410.

Scoville, J.T., R.J. LaHaye, "Design of a Coil to Correct Magnetic Field Errors on the DIII-D Tokamak," in Proc. 14th Symp. on F'usion Engineering, IEEE (San Diego, California, September 30-October 3, 1991) Vol. II (1992) 1144; General Atomics Report GA-A20577 (1991).

Scoville, J.T., R.J. La Haye, A.W. Hyatt, and E.J. Strait, "The Effect of Error Fields on Plasma Stability Near the Beta Limit in DIII-D," in Bull. 34th Annual American Physical Society Meeting, November 16-20, 1992, Seattle, Washington, (Abstract) 37 (1992) 1569.

Seraydarian, R.P., K.H. Burrell, and R.J. Groebner, "A Global Fitting Code for Multichordal Neutral Beam Spectroscopic Data," Rev. Sci. Instrum. 63 (1992) 4764.

Seraydarian, R.P., K.H. Burrell, and R.J. Groebner, "A Global Fitting Code for Multichordal Neutral Beam Spectroscopic Data," in Bull. 34th Annual American Physical Society Meeting, November 16-20, 1992, Seattle, Washington, (Abstract) 37 (1992) 1568.

Simonen, T.C., and the DIII-D Team, "Future Directions of the DIII-D Tokamak Program," to be published in J. of Fusion Energy; General Atomics Report GA-A20914 (1992).

Smith, J.P., C.B. Baxi, E. Reis, M.J. Schaffer, et al., "The Design and Fabrication of a Toroidally Continuous Cryocondensation Pump for the DIII-D Advanced Divertor," in Proc. 14th Symp. on Fusion Engineering, IEEE (San Diego, California, September 30-October 3, 1991) Vol. II (1992) 1230; General Atomics Report GA-A20672 (1991). 
Smith, J.P., C.B. Baxi, E. Reis, and D.L. Sevier, "A Cryocondensation Pump for the DIII-D Advanced Divertor Program," in Proc. 10th Top. Meeting on the Technology of Fusion Energy, June 7-12, 1992, Boston, Massachusetts; General Atomics Report GA-A20851 (1992).

Smith, J.P., M.J. Schaffer, A.W. Hyatt, "The Electrical Insulation of the DIII-D Advanced Divertor Electrode," in Proc. 14th Symp. on Fusion Engineering, IEEE (San Diego, California, September 30-October 3, 1991) Vol. I (1992) 338; General Atomics Report GA-A20623 (1991).

Snider, R.T., and T.N. Carlstrom, "Short Wavelength Interferometer for ITER," Rev. Sci. Instrum. 63 (1992) 4977; General Atomics Report GA-A20855 (1992).

Snider, R.T., R.J. La Haye, and D. Wròblewski, "Modification of the Sawtooth Crash Behavior During Large Error Field Experiments on the DIII-D Tokamak," in Bull. 34th Annual American Physical Society Meeting, November 16-20, 1992, Seattle, Washington, (Abstract) 37 (1992) 1569.

Staebler, G.M., A.W. Hyatt, M.J. Schaffer, and M.A. Mahdavi, "Bias Sustained Shield Plasma for Tokamak Particle Control," in Bull. 34th Annual American Physical Society Meeting, November 16-20, 1992, Seattle, Washington, (Abstract) 37 (1992) 1566.

Staebler, G.M., R.E. Waltz, M. Beer, R.R. Dominguez, et al., "Profile Characteristics of H-Mode Bifurcation Models and Turbulence Simulations With Gyro-Landau Fluid Models in Slab and Toroidal Geometry," to be published in Proc. 14th International Conference on Plasma Physics and Controlled Nuclear Fusion Research, September 30 through October 7, 1992, Wurzburg, Germany; General Atomics Report GA-A21055 (1992).

Stambaugh, R.D., and the DIII-D Team, "DIII-D Research Program Progress," in Proc. 13th International Conference on Plasma Physics and Controlled Nuclear Fusion Research,(October 1-6, 1990, Washington, D.C., Vol. I (1991) p. 69; General Atomics Report GA-A20273 (1990).

Stambaugh, R.D., L.L. Lao, "On the Relation of Vertical Stability and Aspect Ratio in Tokamaks," Nucl. Fusion 32 (1992) 1642; General Atomics Report GA-A20603 (1991).

Stambaugh, R.D., Q. Nguyen, R.J. Groebner, K.H. Burrell, and P. Gohil, "Edge Ion Orbit Loss and H-Mode," in Bull. 34th Annual American Physical Society Meeting, November 16-20, 1992, Seattle, Washington, (Abstract) 37 (1992) 1509. 
St. John, H., L.L. Lao, R.A. James, Y.R. Lin-Liu, and S.J. Thompson, "Self-Consistent MHD/Transport Modeling of Some DIII-D Discharges," in Bull. 34th Annual American Physical Society Meeting, November 16-20, 1992, Seattle, Washington, (Abstract) 37 (1992) 1510.

Strait, E.J., V.S. Chan, M.S. Chu, J.R. Ferron, et al., "The Influence of Current and Pressure Profiles on Operational Limits in DIII-D," General Atomics Report GA-C21066 (1992).

Strait, E.J., W.W. Heidbrink, M.S. Chu, H.H. Duong, et al., "Stability of TAE Modes in DIII-D," to be published in Proc. 14th International Conference on Plasma Physics and Controlled Nuclear Fusion Research, September 30 through October 7, 1992, Wurzburg, Germany; General Atomics Report GA-A21045 (1992).

Strait, E.J., W.W. Heidbrink, M.S. Chu, H.H. Duong, et al., "Stability of TAE Modes in DII-D," in Bull. 34th Annual American Physical Society Meeting, November 16-20, 1992, Seattle, Washington, (Abstract) 37 (1992) 1410.

Strait, E.J., W.W. Heidbrink, M.S. Chu, H.H. Duong, et al., "Stability of TAE Modes in DIII-D," in special issue Phys. Fluids B (1993); General Atomics Report GA-A21045 (1992).

Taylor, P.L., "DIII-D Radiation Shielding Procedures and Experiences," in Proc. 14th Symp. on Fusion Engineering, IEEE (San Diego, California, September 30-October 3, 1991) Vol. I (1992) 617; General Atomics Report GA-A20570 (1991).

Taylor, T.S., T.H. Osborne, K.H. Burrell, T.N. Carlstrom, et al., "Confinement and Stability of VH-Mode Discharges in the DIII-D Tokamak," in Proc. 14th International Conference on Plasma Physics and Controlled Nuclear Fusion Research, September 30 through October 7, 1992, Wurzburg, Germany; General Atomics Report GA-A21092 (1992).

Taylor, P.L., A.G. Kellman, K. Holtrop, and R.L. Lee, "DIII-D Disruption Database and Recent Results," in Bull. 34th Annual American Physical Society Meeting, November 16-20, 1992, Seattle, Washington, (Abstract) 37 (1992) 1570.

Thomas, D.M., R.M. Patterson, W.B. McHarg, E. McKee, "Initial Results From the DIII-D Lithium Beam Diagnostic," in Bull. 34th Annual American Physical Society Meeting, November 16-20, 1992, Seattle, Washington, (Abstract) 37 (1992) 1568.

Thomas, M.P., T.N. Carlstrom, J.C. DeBoo, J. Evans, et al., "Measurement Calibration Studies on the DIII-D Eight Laser Multipulse Thomson Scattering Diagnostic," in Bull. 34th Annual American Physical Society Meeting, November 16-20, 1992, Seattle, Washington, (Abstract) 37 (1992) 1567. 
GA-A21186; FY92 DIII-D Research Operations Annual Report

Thompson, S.J., L.L. Lao, J.R. Ferron, and H.E. St. John, "A Portable Version of EFIT With Toroidal Rotation for VMS and UNIX Environments," in Bull. 34th Annual American Physical Society Meeting, November 16-20, 1992, Seattle, Washington, (Abstract) 37 (1992) 1511.

Wade, M.R., D.L. Hillis, J.T. Hogan, D.F. Finkenthal, et al., "Helium Transport Studies in DIII-D," in Bull. 34th Annual American Physical Society Meeting, November 16-20, 1992, Seattle, Washington, (Abstract) 37 (1992) 1411.

Waltz, R.E., and A.H. Boozer, "Local Shear in General Magnetic Stellarator Geometry," submitted to Phys. Fluids B ; General Atomics Report GA-A21071 (1992).

Waltz, R.E., J.C. DeBoo, and T.H. Osborne, "Modeling Almost Dimensionally Similar Discharges," Nucl. Fusion 32 (1992) 1051; General Atomics Report GA-A20705 (1992).

Watkins, J.G., R.A. Moyer, J.W. Cuthbertson, D. Buchenauer, et al., "Characterization of the DIII-D Boundary Plasma," in Bull. 34th Annual American Physical Society Meeting, November 16-20, 1992, Seattle, Washington, (Abstract) 37 (1992) 1411.

West, W.P., S.I. Lippmann, N.H. Brooks, A.M. Howald, et al., "Impurity Influx on DIII-D," in Bull. 34th Annual American Physical Society Meeting, November 16-20, 1992, Seattle, Washington, (Abstract) 37 (1992) 1566.

Wight, J., R.M. Hong, J. Phillips, "Recent DIII-D Neutral Beam Calibration Results," in Proc. 14th Symp. on Fusion Engineering, IEEE (San Diego, California, September 30-October 3, 1991) Vol. I (1992) 66; General Atomics Report GA-A20650 (1991).

Wròblewski, D., and R.T. Snider, "A Study of Current Profiles in Sawtoothing Discharges in DIII-D," in Bull. 34th Annual American Physical Society Meeting, November 16-20, 1992, Seattle, Washington, (Abstract) 37 (1992) 1569.

Wròblewski, D., and L.L. Lao, "Polarimetry of Motional Stark Effect and Determination of Current Profiles in DIII-D," Rev. Sci. Instrum. 63 (1992) 5160; General Atomics Report GA-A20881 (1992).

Zohm, H., J.M. Greene, L.L. Lao, and E.J. Strait, "Mirnov Coil Analysis in DIII-D Tokamak Using the Singular Value Decomposition Method," to be published in Nucl. Fusion; General Atomics Report GA-A20886 (1992). 